\title{
Electrostatic Effects on the Reactions of Cyclohexanone Oxocarbenium Ions
}

\author{
Glen Baghdasarian and K. A. Woerpel \\ Contribution from the Department of Chemistry, University of California, Irvine, \\ California 92697-2025
}

Table of Contents

I. Experimental Section

$S-2$

A. General Experimental

$S-2$

B. Synthesis of Substrates

$S-2$

C. Featured Reactions in Corresponding Article

$S-12$

D. Proofs of Stereochemistry

$S-22$

II. Calculations of the Structure of 4-Alkoxy-Substituted Oxocarbenium Ions $S$-29

III. X-Ray Crystallographic Data

$S-31$

IV. Proofs of Diastereoselectivity

$S-105$

V. Correlation of $6 b$ and $6 b$ '

$S-147$

VI. Proofs of Purity

S-149 


\section{Experimental Section}

\section{A. General Experimental}

${ }^{1} \mathrm{H}$ NMR and ${ }^{13} \mathrm{C}$ NMR spectra were recorded at ambient temperature at $500 \mathrm{MHz}$ and $125 \mathrm{MHz}$. The data are reported as follows: chemical shift in ppm from internal tetramethylsilane on the $\delta$ scale, multiplicity $(\mathrm{br}=$ broad, $\mathrm{s}=$ singlet, $\mathrm{d}=$ doublet, $\mathrm{t}=$ triplet, $\mathrm{q}=$ quartet, $\mathrm{m}=$ multiplet $)$, coupling constants $(\mathrm{Hz})$, and integration. High resolution mass spectra were obtained by peak matching. Analytical gas-liquid chromatography (GC) analyses were performed with a fused silica capillary column (30 $\mathrm{m} \times 0.32 \mathrm{~mm}$ ) wall coated with DB-1 with helium as the carrier gas (16 psi column head pressure). Method of chromatography is as follows: Start temperature $-50{ }^{\circ} \mathrm{C}$; Ramp $10{ }^{\circ} \mathrm{C} / \mathrm{min}$; Final Temp $-250^{\circ} \mathrm{C}$. Analytical gas-liquid chromatography mass spectrometry (GCMS) was performed in EI mode equipped with a split-less mode injection system. A fused silica capillary column was coated with DB-5 (30 m $\times 0.32$ $\mathrm{mm}$ ID). Method of chromatography is as follows: Start temperature $-50{ }^{\circ} \mathrm{C}$; Ramp -10 ${ }^{\circ} \mathrm{C} / \mathrm{min}$; Final Temp $-250{ }^{\circ} \mathrm{C}$. Melting points are reported uncorrected. Liquid chromatography was performed using forced flow (flash chromatography) of the indicated solvent system on silica gel $\left(\mathrm{SiO}_{2}\right) 60$ (230-400 mesh). All reactions were carried out under an atmosphere of nitrogen in glassware that had been flame-dried under a stream of nitrogen. Unless otherwise noted, all reagents were commercially obtained and, where appropriate, purified prior to use. THF, $\mathrm{Et}_{2} \mathrm{O}$, toluene, and $\mathrm{CH}_{2} \mathrm{Cl}_{2}$ were dried by filtration through alumina according to the procedure of Grubbs ${ }^{1}$.

\section{B. Synthesis of Substates}

1. Synthesis of ketal $\mathbf{3 b}$

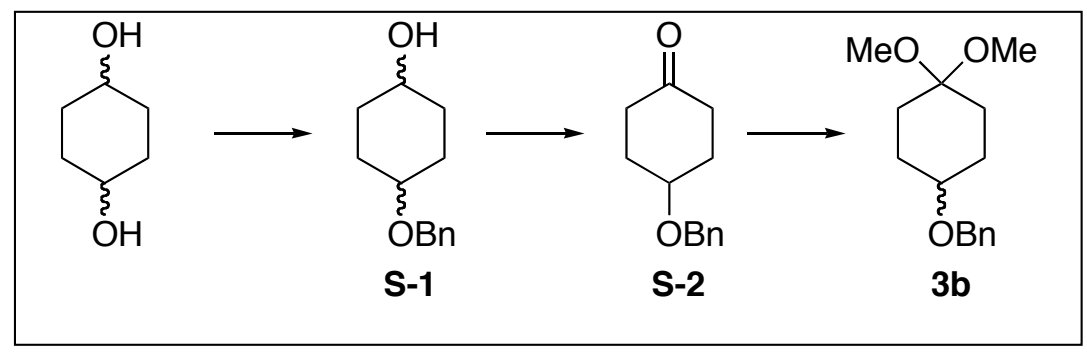<smiles>O[C@H]1CC[C@H](OCc2ccccc2)CC1</smiles>

S-1

Alcohol S-1 ${ }^{2}$ : To a solution of 1,4 cyclohexane diol (5.9 g, $\left.50 \mathrm{mmol}\right)$ and $\mathrm{NaH}(60 \%$ dispersion in oil, $2.4 \mathrm{~g}, 60 \mathrm{mmol})$ in DMF $(125 \mathrm{~mL})$ was added benzyl bromide $(7.2 \mathrm{~mL}$, $60 \mathrm{mmol})$. The mixture was stirred for 1 day and $\mathrm{H}_{2} \mathrm{O}(200 \mathrm{~mL})$ was added along with methyl $t$-butyl ether $(200 \mathrm{~mL})$. The layers were separated and the aqueous layer was extracted with methyl $t$-butyl ether $(5 \times 200 \mathrm{~mL})$. The organic layers were combined, filtered through $\mathrm{Na}_{2} \mathrm{SO}_{4}$, and concentrated in vacuo. The resulting oil was purified by 
flash chromatography (80:20 to $50: 50$ hexanes/EtOAc) to afford the product (a 50:50 mixture of diastreomers as determined by ${ }^{1} \mathrm{H}$ NMR) as a colorless oil $(3.4 \mathrm{~g}, 32 \%)$ : ${ }^{1} \mathrm{H}$ NMR (500 MHz, $\left.\mathrm{CDCl}_{3}\right), \delta$ 7.37-7.30 (m, 8H), 7.28-7.24 (m, 2H), $4.52(\mathrm{~s}, 2 \mathrm{H}), 4.50(\mathrm{~s}$, $2 \mathrm{H}), 3.76-3.58(\mathrm{~m}, 2 \mathrm{H}), 3.47(\mathrm{dq}, J=9.1,3.1,1 \mathrm{H}), 3.35$ (tt, $J=9.7,3.9,1 \mathrm{H}), 2.30-2.10$ $(\mathrm{m}, 4 \mathrm{H}), 1.94-1.85(\mathrm{~m}, 4 \mathrm{H}), 1.74-1.67(\mathrm{~m}, 2 \mathrm{H}), 1.64-1.60(\mathrm{~m}, 2 \mathrm{H}), 1.57-1.50(\mathrm{~m}, 2 \mathrm{H})$, 1.37 (tdd, $J=12.5,9.8,3.0,2 \mathrm{H}), 1.28(\mathrm{tdd}, J=12.8,9.5,3.1,2 \mathrm{H}) ;{ }^{13} \mathrm{C}$ NMR $(125 \mathrm{MHz}$, $\left.\mathrm{CDCl}_{3}\right), \delta 139.0,138.8,128.22,128.18,127.4,127.32,127.29,127.2,76.1,73.4,70.0$, $69.5,69.3,68.2,32.5,30.3,29.3,27.4$.

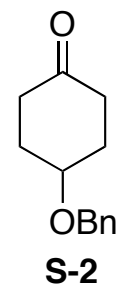

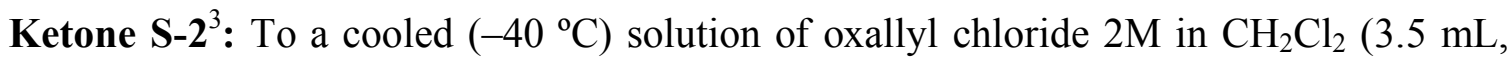
$6.9 \mathrm{mmol})$ in $\mathrm{CH}_{2} \mathrm{Cl}_{2}(20 \mathrm{~mL})$ was slowly added a solution of dimethyl sulfoxide $(0.85$ $\mathrm{mL}, 12 \mathrm{mmol})$ in $\mathrm{CH}_{2} \mathrm{Cl}_{2}(20 \mathrm{~mL})$. The mixture was allowed to warm $\left(0{ }^{\circ} \mathrm{C}\right)$ and cooled $\left(-40^{\circ} \mathrm{C}\right)$ again. To the above mixture was added $\mathbf{S}-1(1.2 \mathrm{~g}, 5.8 \mathrm{mmol})$ and the mixture stirred for 20 minutes. The mixture was then warmed $\left(0{ }^{\circ} \mathrm{C}\right)$ and stirred for an additional 20 minutes. $\mathrm{Et}_{3} \mathrm{~N}(4.1 \mathrm{~mL}, 41 \mathrm{mmol})$ was added to the mixture and stirred for 2 hours. $\mathrm{H}_{2} \mathrm{O}(30 \mathrm{~mL})$ was added to the mixture. The layers were separated and the aqueous layer was extracted with $\mathrm{CH}_{2} \mathrm{Cl}_{2}(5 \times 30 \mathrm{~mL})$. The organic layers were combined, filtered through $\mathrm{Na}_{2} \mathrm{SO}_{4}$, and concentrated in vacuo. The resulting oil was purified by flash chromatography (80:1 hexanes/EtOAc) to afford the product as colorless oil (1.2 g, 99\%): ${ }^{1} \mathrm{H}$ NMR $\left(500 \mathrm{MHz}, \mathrm{CDCl}_{3}\right)$ 7.35-7.31 (m, 4H), 7.28-7.25 (m, 1H), $4.57(\mathrm{~s}, 2 \mathrm{H}), 3.79(\mathrm{tt}$, $J=5.7,2.9,1 \mathrm{H}), 2.59$ (ddd, $J=15.5,10.5,5.8,2 \mathrm{H}), 2.24$ (dt, $J=14.6,5.8,2 \mathrm{H}), 2.11$ (dq, $J=11.6,5.8,2 \mathrm{H}), 1.92(\mathrm{~m}, 2 \mathrm{H}) ;{ }^{13} \mathrm{C}$ NMR $\left(125 \mathrm{MHz}, \mathrm{CDCl}_{3}\right), \delta$ 210.6, 138.3, 128.1, 127.3, 127.1, 72.0, 69.9, 36.9, 30.2; HRMS (EI) $m / z$ calcd for $\mathrm{C}_{13} \mathrm{H}_{16} \mathrm{O}_{2} \mathrm{Na}(\mathrm{M}+\mathrm{Na})^{+}$ 227.1048 , found 227.1045 .

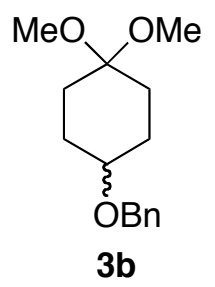

Ketal 3b: To a solution of S-2 $(1.1 \mathrm{~g}, 5.5 \mathrm{mmol})$ in methanol $(30 \mathrm{~mL})$ was added trimethyl orthoformate $(4.6 \mathrm{~mL}, 42 \mathrm{mmol})$ and $p$-toluesulfonic acid $(0.030 \mathrm{~g}, 0.16 \mathrm{mmol})$. The reaction mixture was stirred for 1 day, then $\mathrm{NaHCO}_{3}$ (30 mL saturated, aqueous) was added along with $\mathrm{CH}_{2} \mathrm{Cl}_{2}(30 \mathrm{~mL})$. The layers were separated and the aqueous layer was extracted with $\mathrm{CH}_{2} \mathrm{Cl}_{2}(5 \times 30 \mathrm{~mL})$. The organic layers were combined, filtered through $\mathrm{Na}_{2} \mathrm{SO}_{4}$, and concentrated in vacuo. The resulting oil was purified by flash chromatography (95:5 hexanes/EtOAc) to afford the product as a colorless oil (1.3 g, 91\%): ${ }^{1} \mathrm{H}$ NMR $\left(500 \mathrm{MHz}, \mathrm{CDCl}_{3}\right) \quad \delta$ 7.34-7.32 (m, 4H), 7.28-7.25 (m, 1H), $4.53(\mathrm{~s}$, 
2H), $3.50(\mathrm{dq}, J=11.1,3.61 \mathrm{H}), 3.19$ (s, 3H), $3.18(\mathrm{~s}, 3 \mathrm{H}), 1.92$ (ddd, $J=12.0,7.8,3.8$, $2 \mathrm{H}$ ), 1.78 (ddd, $J=12.2,7.7,3.9,2 \mathrm{H}$ ), $1.72-1.65$ (m, 2H), 1.55 (ddd, $J=13.3,7.9,4.5$, $2 \mathrm{H}) ;{ }^{13} \mathrm{C}$ NMR (125 MHz, $\left.\mathrm{CDCl}_{3}\right) \delta 139.2,128.3,127.4,127.3,99.6,74.8,70.0,47.8$, 47.4, 29.0, 27.6; IR (thin film) 2940, 1447, $11001075 \mathrm{~cm}^{-1}$; HRMS (CI, $\mathrm{NH}_{4} \mathrm{Cl}$ ) $\mathrm{m} / \mathrm{z}$ calcd for $\mathrm{C}_{15} \mathrm{H}_{22} \mathrm{O}_{3}(M)^{+}$250.1569, found 250.1566. Anal. Calcd for $\mathrm{C}_{15} \mathrm{H}_{22} \mathrm{O}_{3}$ : C, 71.97; H, 8.86. Found: C, 71.95; H, 8.94.

2. Synthesis of ketal $\mathbf{3 a}$

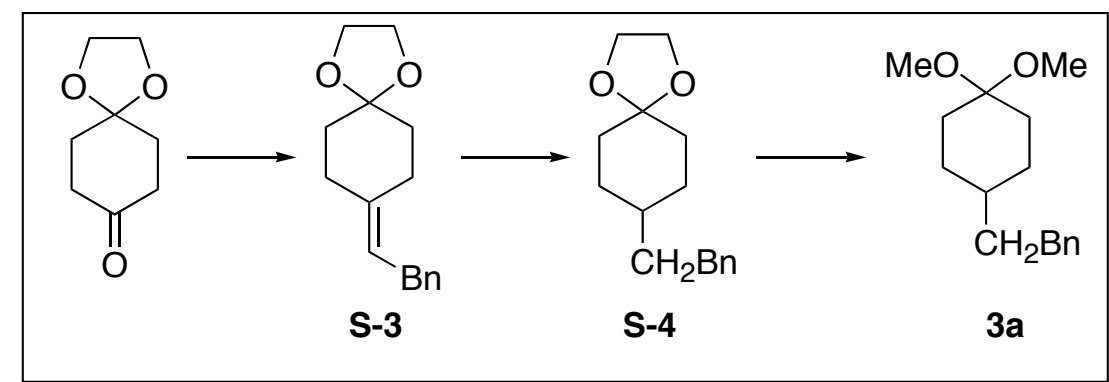<smiles>C(=C1CCC2(CC1)OCCO2)c1ccccc1</smiles>

S-3

Alkene S-3: To a cooled $\left(-78{ }^{\circ} \mathrm{C}\right)$ solution of phenethyltriphenylphosphonium bromide $(2.58 \mathrm{~g}, 5.80 \mathrm{mmol}$, prepared by stirring neat triphenylphosphine with phenethyl bromide at $90{ }^{\circ} \mathrm{C}$ for 24 hours) in THF $(40 \mathrm{~mL})$ was slowly added a $1.6 \mathrm{M}$ solution of $n$ butyllithium in hexanes $(3.60 \mathrm{~mL}, 5.80 \mathrm{mmol})$. The mixture was allowed to warm $(23$ $\left.{ }^{\circ} \mathrm{C}\right)$ and was stirred for 1 hour then cooled $\left(-78{ }^{\circ} \mathrm{C}\right)$ again. A solution of 1,4 cyclohexanone ethylene glycol ketal $(0.56 \mathrm{~g}, 3.6 \mathrm{mmol})$ in THF $(10 \mathrm{~mL})$ was then added. The mixture was allowed to warm $\left(23{ }^{\circ} \mathrm{C}\right)$ and stirred for 15 hours. Then $\mathrm{NaHCO}_{3}(20$ $\mathrm{mL}$ saturated., aqueous) was added along with $\mathrm{CH}_{2} \mathrm{Cl}_{2}(30 \mathrm{~mL})$. The layers were separated and the aqueous layer was extracted with $\mathrm{CH}_{2} \mathrm{Cl}_{2}(5 \times 30 \mathrm{~mL})$. The organic layers were combined, filtered through $\mathrm{Na}_{2} \mathrm{SO}_{4}$, and concentrated in vacuo. The resulting oil was purified by flash chromatography (95:5 to $94: 6$ hexanes/EtOAc) to afford the product as a colorless oil $(0.69 \mathrm{~g}, 78 \%):{ }^{1} \mathrm{H}$ NMR $\left(500 \mathrm{MHz}, \mathrm{CDCl}_{3}\right) \delta$ 7.29-7.25 (m, $2 \mathrm{H}), 7.18-7.14(\mathrm{~m}, 3 \mathrm{H}), 5.36-5.32(\mathrm{~m}, 1 \mathrm{H}), 3.96-3.92(\mathrm{~m}, 4 \mathrm{H}), 3.35(\mathrm{~d}, J=7.5,2 \mathrm{H})$, 2.40-2.37 (m, 2H), 2.29-2.26 (m, 2H), 1.71-1.69 (m, 4H); ${ }^{13} \mathrm{C}$ NMR (125 MHz, $\left.\mathrm{CDCl}_{3}\right)$, $\delta 141.4,137.7,128.3,125.7,121.3,108.9,64.2,36.1,35.4,33.6,33.5,25.1$; IR (thin film) 3356, 2936, 2881, 1602, 1491, $1268 \mathrm{~cm}^{-1}$. Anal. Calcd for $\mathrm{C}_{16} \mathrm{H}_{20} \mathrm{O}_{2}: \mathrm{C}, 78.65 ; \mathrm{H}$, 8.25. Found: C, 78.63; H, 8.29. 


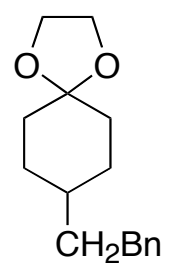

S-4

Ketal S-4: To a solution of S-3 $(0.42 \mathrm{~g}, 1.7 \mathrm{mmol})$ in ethyl acetate $(5 \mathrm{~mL})$ was added $\mathrm{PtO}_{2}(0.01 \mathrm{~g}, 0.09 \mathrm{mmol})$. The mixture was purged with $\mathrm{H}_{2}$ and stirred under $\mathrm{H}_{2}(1 \mathrm{~atm})$ for 1 day. The mixture was filtered and the solvent was removed in vacuo. The resulting oil was purified by flash chromatography (98:2 to $97: 3$ hexanes/EtOAc) to afford the product as a colorless oil $(0.15 \mathrm{~g}, 36 \%)$ : ${ }^{1} \mathrm{H} \mathrm{NMR}\left(500 \mathrm{MHz}, \mathrm{CDCl}_{3}\right), \delta$ 7.28-7.23 (m, $2 \mathrm{H}), 7.12-7.15(\mathrm{~m}, 3 \mathrm{H}), 3.95-3.92(\mathrm{~m}, 4 \mathrm{H}), 2.63-2.60(\mathrm{~m}, 2 \mathrm{H}), 1.78-1.72(\mathrm{~m}, 4 \mathrm{H}), 1.58-$ $1.48(\mathrm{~m}, 4 \mathrm{H}), 1.37-1.22(\mathrm{~m}, 3 \mathrm{H}) ;{ }^{13} \mathrm{C}$ NMR $\left(125 \mathrm{MHz}, \mathrm{CDCl}_{3}\right) \delta 142.8,128.23,128.22$, 125.5, 109.1, 64.1, 38.1, 35.9, 34.4, 33.5, 30.0; IR (thin film) 2922, 2833, 1469, $1367 \mathrm{~cm}^{-}$ ${ }^{1}$; HRMS (ES) $m / z$ calcd for $\mathrm{C}_{16} \mathrm{H}_{23} \mathrm{O}_{2}(\mathrm{M}+\mathrm{H})^{+} 247.1698$, found 247.1688. Anal. Calcd for $\mathrm{C}_{16} \mathrm{H}_{22} \mathrm{O}_{2}$ : C, 78.01; H, 9.00. Found: C, 77.78; H, 9.21.<smiles>COC1(OC)CCC(Cc2ccccc2)CC1</smiles>

$3 a$

Ketal 3a: To a solution of S-4 $(1.2 \mathrm{~g}, 4.7 \mathrm{mmol})$ in a mixture of THF and $\mathrm{H}_{2} \mathrm{O}(10 \mathrm{~mL}$, 4:1 THF/ $\mathrm{H}_{2} \mathrm{O}$ ) was added 1 drop of $\mathrm{H}_{2} \mathrm{SO}_{4}$ (concentrated). The mixture was heated (75 $\left.{ }^{\circ} \mathrm{C}\right)$ for 1 day. Then $\mathrm{NaHCO}_{3}\left(5 \mathrm{~mL}\right.$ saturated, aqueous) was added along with $\mathrm{CH}_{2} \mathrm{Cl}_{2}$ $(10 \mathrm{~mL})$. The layers were separated, and the aqueous layer was extracted with $\mathrm{CH}_{2} \mathrm{Cl}_{2}(5$ x $10 \mathrm{~mL}$ ). The organic layers were combined, filtered through $\mathrm{Na}_{2} \mathrm{SO}_{4}$, and concentrated in vacuo. The resulting oil was then used in the next reaction without purification. To a solution of the unpurified ketone (from the previous step) in methanol $(10 \mathrm{~mL})$ was added trimethyl orthoformate $(3 \mathrm{~mL}, 0.27 \mathrm{mmol})$ and $p$-toluesulfonic acid $(0.030 \mathrm{~g}, 0.16 \mathrm{mmol})$. The mixture was stirred for 1 day. $\mathrm{NaHCO}_{3}(5 \mathrm{~mL}$ saturated, aqueous) was added along with $\mathrm{CH}_{2} \mathrm{Cl}_{2}(10 \mathrm{~mL})$. The layers were separated and the aqueous layer was extracted with $\mathrm{CH}_{2} \mathrm{Cl}_{2}(5 \times 10 \mathrm{~mL})$. The organic layers were combined, filtered through $\mathrm{Na}_{2} \mathrm{SO}_{4}$, and concentrated in vacuo. The resulting oil was purified by flash chromatography (98:2 hexanes/EtOAc) to afford the product as colorless oil $(0.99 \mathrm{~g}, 84 \%$ over two reactions $)$ : ${ }^{1} \mathrm{H}$ NMR $\left(500 \mathrm{MHz}, \mathrm{CDCl}_{3}\right) \delta$ 7.28-7.24 (m, 2H), 7.17-7.12 (m, 3H), $3.19(\mathrm{~s}, 3 \mathrm{H}), 3.15$ $(\mathrm{s}, 3 \mathrm{H}), 2.61(\mathrm{~m}, 2 \mathrm{H}), 1.99(\mathrm{tdd}, J=6.1,6.0,3.6,2 \mathrm{H}), 1.68(\mathrm{~m}, 2 \mathrm{H}), 1.55(\mathrm{dd}, J=16.0$, $6.8,2 \mathrm{H}), 1.32(\mathrm{ddd}, J=13.4,13.3,3.7,3 \mathrm{H}), 1.16(\mathrm{ddd}, J=15.6,13.3,3.1,2 \mathrm{H}) ;{ }^{13} \mathrm{C} \mathrm{NMR}$ $\left(125 \mathrm{MHz}, \mathrm{CDCl}_{3}\right) \delta 142.9,128.3,128.2,125.6,100.1,47.5,47.3,38.2,36.4,33.5,32.0$, 28.99; HRMS (EI) $m / z$ calcd for $\mathrm{C}_{15} \mathrm{H}_{21} \mathrm{O}\left(\mathrm{M}-\mathrm{CH}_{3} \mathrm{O}\right)^{+}$217.1592, found 217.1600. Anal. Calcd for $\mathrm{C}_{16} \mathrm{H}_{24} \mathrm{O}_{2} \mathrm{C}, 77.38 ; \mathrm{H}, 9.74$. Found: C, 77.18; H, 9.75. 
3. Synthesis of ketal $\mathbf{3 c}$

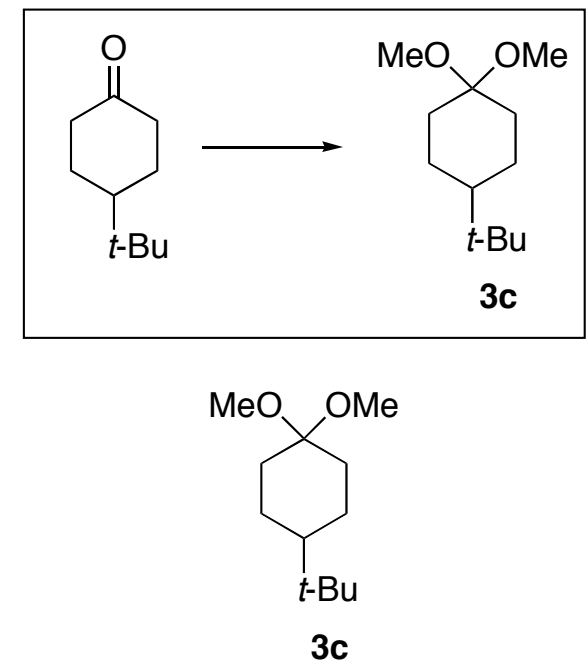

Ketal $3 \mathbf{c}^{4}$ : To a solution of 4-t-butyl cyclohexanone $(1.0 \mathrm{~g}, 6.5 \mathrm{mmol})$ in methanol (30 $\mathrm{mL}$ ) was added trimethyl orthoformate $(5.5 \mathrm{~mL}, 50 \mathrm{mmol})$ and $p$-toluesulfonic acid $(0.035 \mathrm{~g}, 0.18 \mathrm{mmol})$. The reaction was stirred for 1 day, then $\mathrm{NaHCO}_{3}(30 \mathrm{~mL}$ saturated, aqueous) was added along with $\mathrm{CH}_{2} \mathrm{Cl}_{2}(30 \mathrm{~mL})$. The layers were separated and the aqueous layer was extracted with $\mathrm{CH}_{2} \mathrm{Cl}_{2}(5 \times 30 \mathrm{~mL})$. The organic layers were combined, filtered through $\mathrm{Na}_{2} \mathrm{SO}_{4}$, and concentrated in vacuo to afford the product as a colorless oil (1.3 g, 98\%): ${ }^{1} \mathrm{H}$ NMR $\left(500 \mathrm{MHz}, \mathrm{CDCl}_{3}\right), \delta 3.30(\mathrm{~s}, 3 \mathrm{H}), 3.15(\mathrm{~s}, 3 \mathrm{H})$, 2.08-2.03 (m, 2H), 1.27 (ddd, $J=13.2,13.0,3.8,2 \mathrm{H}), 1.19-1.11(\mathrm{~m}, 2 \mathrm{H}), 1.16$ (ddd, $J=$ 16.7, 12.7, 3.0, 2H), 1.03-0.97 (m, 1H), $0.86(\mathrm{~s}, 9 \mathrm{H}) ;{ }^{13} \mathrm{C} \mathrm{NMR}\left(125 \mathrm{MHz}, \mathrm{CDCl}_{3}\right) \delta$ 99.9, 47.6, 47.6, 47.4, 32.8, 32.3, 27.7, 23.6; HRMS (EI) $m / z$ calcd for $\mathrm{C}_{12} \mathrm{H}_{24} \mathrm{O}_{2}(\mathrm{M})^{+}$ 200.1776 , found 200.1777 .

4. Synthesis of ketal 23

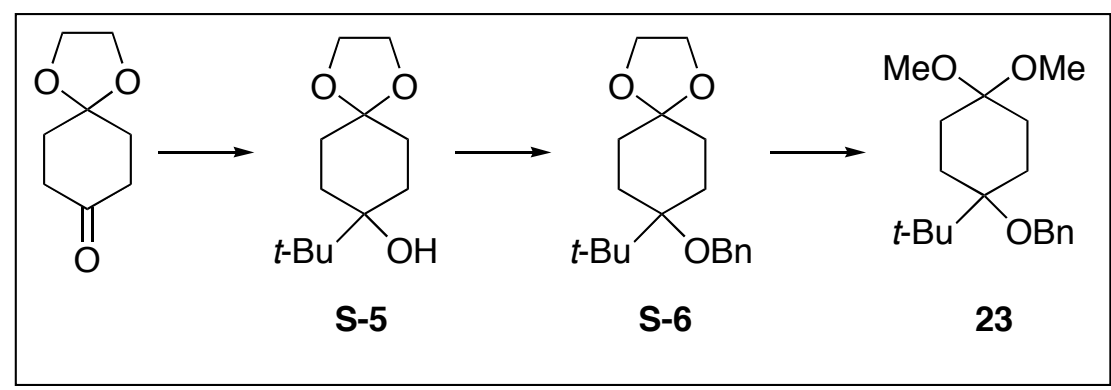<smiles>OC1([18F])CCC2(CC1)OCCO2</smiles> 
Alcohol S-5: To a cooled $\left(-78^{\circ} \mathrm{C}\right)$ solution of $\mathrm{CeCl}_{3}(0.600 \mathrm{~g}, 1.66 \mathrm{mmol})$ in THF (5 $\mathrm{mL})$ was added a $t$-butyl lithium solution $(1.7 \mathrm{M}$ in hexanes, $0.730 \mathrm{~mL}, 1.66 \mathrm{mmol})$. The mixture was stirred for 1 hour then a solution of 1,4 cyclohexanone ethylene glycol ketal $(0.193 \mathrm{~g}, 0.122 \mathrm{mmol})$ in THF $(2 \mathrm{~mL})$ was added and the mixture was stirred for 1 hour. The mixture was allowed to warm $\left(23^{\circ} \mathrm{C}\right)$ and $\mathrm{NH}_{4} \mathrm{Cl}(10 \mathrm{~mL}$, saturated, aqueous) was added along with $\mathrm{CH}_{2} \mathrm{Cl}_{2}(10 \mathrm{~mL})$. The layers were separated and the aqueous layer was extracted with $\mathrm{CH}_{2} \mathrm{Cl}_{2}(5 \times 10 \mathrm{~mL})$. The organic layers were combined, filtered through $\mathrm{Na}_{2} \mathrm{SO}_{4}$, and concentrated in vacuo. The resulting oil contained 1,4 cyclohexanone ethylene glycol ketal and so the following measure was taken before purification: To the resulting oil was then added THF $(5 \mathrm{~mL})$ and the mixture was cooled $\left(0{ }^{\circ} \mathrm{C}\right) . \mathrm{LiAlH}_{4}$ $(0.200 \mathrm{~g}, 5.31 \mathrm{mmol})$ was added and the mixture was warmed $\left(23{ }^{\circ} \mathrm{C}\right)$ and cooled $\left(0{ }^{\circ} \mathrm{C}\right)$ again. Ethanol $(3 \mathrm{~mL})$ was added slowly along with $\mathrm{CH}_{2} \mathrm{Cl}_{2}(5 \mathrm{~mL})$. The layers were separated and the aqueous layer was extracted with $\mathrm{CH}_{2} \mathrm{Cl}_{2}(5 \times 5 \mathrm{~mL})$. The organic layers were combined, filtered through $\mathrm{Na}_{2} \mathrm{SO}_{4}$, and concentrated in vacuo. The resulting oil was then purified by flash chromatography (83:17 hexanes/EtOAc) to afford the product as a colorless oil $(0.07 \mathrm{~g}, 26 \%):{ }^{1} \mathrm{H}$ NMR $\left(500 \mathrm{MHz}, \mathrm{CDCl}_{3}\right) \delta 3.97-3.91(\mathrm{~m}$, $4 \mathrm{H}), 1.92(\mathrm{td}, J=13.4,4.2,2 \mathrm{H}), 1.77(\mathrm{ddd}, J=13.8,13.7,3.8,2 \mathrm{H}), 1.68-1.57(\mathrm{~m}, 4 \mathrm{H})$, 1.18 (br s, $1 \mathrm{H}), 0.95$ (s, 9H); ${ }^{13} \mathrm{C}$ NMR $\left(125 \mathrm{MHz}, \mathrm{CDCl}_{3}\right) \delta$ 108.7, 74.2, 64.14, 64.09, 37.4, 30.3, 28.7, 25.2; IR (thin film) 3509, 2965, 1372, $1096 \mathrm{~cm}^{-1}$. Anal. Calcd for $\mathrm{C}_{12} \mathrm{H}_{22} \mathrm{O}_{3}$ : C, 67.26; $\mathrm{H}, 10.35$. Found: $\mathrm{C}, 67.44 ; \mathrm{H}, 10.29$.

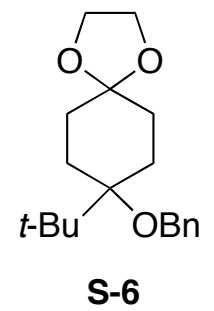

Ketal S-6: To a cooled $\left(0{ }^{\circ} \mathrm{C}\right)$ solution of S-5 $(0.69 \mathrm{~g}, 3.2 \mathrm{mmol})$ in THF $(10 \mathrm{~mL})$ was added $\mathrm{KH}(0.14 \mathrm{~g}, 3.6 \mathrm{mmol})$ and the mixture was stirred for 18 hours. Benzyl bromide $(1.2 \mathrm{~mL}, 9.7 \mathrm{mmol})$ was added and the mixture was stirred for 3 days. Then $\mathrm{NaHCO}_{3}(5$ $\mathrm{mL}$ saturated, aqueous) was added along with $\mathrm{CH}_{2} \mathrm{Cl}_{2}(10 \mathrm{~mL})$. The layers were separated and the aqueous layer were extracted with $\mathrm{CH}_{2} \mathrm{Cl}_{2}(5 \times 10 \mathrm{~mL})$. The organic layers were combined, filtered through $\mathrm{Na}_{2} \mathrm{SO}_{4}$, and concentrated in vacuo. The resulting oil was purified by flash chromatography (97:3 to $91: 9$ hexanes/EtOAc) to afford the product as a white solid $(0.79 \mathrm{~g}, 80 \%): \mathrm{mp} 46{ }^{\circ} \mathrm{C}$; ${ }^{1} \mathrm{H}$ NMR $\left(500 \mathrm{MHz}, \mathrm{CDCl}_{3}\right) \delta$ 7.36$7.34(\mathrm{~m}, 2 \mathrm{H}), 7.32-7.29(\mathrm{~m}, 2 \mathrm{H}), 7.23-7.19(\mathrm{~m}, 1 \mathrm{H}), 4.62(\mathrm{~s}, 2 \mathrm{H}), 3.90(\mathrm{~s}, 4 \mathrm{H}), 2.01-1.96$ $(\mathrm{m}, 2 \mathrm{H}), 1.87$ (ddd, $J=13.5,13.4,3.4,2 \mathrm{H}), 1.79-1.72(\mathrm{~m}, 2 \mathrm{H}), 1.59-1.57(\mathrm{~m}, 2 \mathrm{H}), 1.03$ $(\mathrm{s}, 9 \mathrm{H}) ;{ }^{13} \mathrm{C} \mathrm{NMR}\left(125 \mathrm{MHz}, \mathrm{CDCl}_{3}\right) \delta 139.9,128.2,126.9,126.7,108.9,79.0,65.9$, 64.1, 64.0, 38.4, 30.7, 27.2, 26.7; IR (KBr) 2960, 2876, 2360, 2340, 1374, $1103 \mathrm{~cm}^{-1}$. Anal. Calcd for $\mathrm{C}_{19} \mathrm{H}_{28} \mathrm{O}_{3}$ : C, 74.96; H, 9.27. Found: C, 75.18; H, 9.31. 
<smiles>COC1(OC)CCC(Cc2ccccc2)(OC(C)(C)C)CC1</smiles>

Ketal 23: To a solution of S-6 $(0.79 \mathrm{~g}, 2.6 \mathrm{mmol})$ in a mixture of THF: $\mathrm{H}_{2} \mathrm{O}(4: 1,10 \mathrm{~mL})$ was added 1 drop $\mathrm{H}_{2} \mathrm{SO}_{4}$, (concentrated). The mixture was cooled $\left(23^{\circ} \mathrm{C}\right)$ and $\mathrm{NaHCO}_{3}$ $\left(10 \mathrm{~mL}\right.$, saturated, aqueous) was added along with $\mathrm{CH}_{2} \mathrm{Cl}_{2}(10 \mathrm{~mL})$. The layers were separated and the aqueous layer was extracted with $\mathrm{CH}_{2} \mathrm{Cl}_{2}(5 \times 10 \mathrm{~mL})$. The organic layers were combined, filtered through $\mathrm{Na}_{2} \mathrm{SO}_{4}$, and concentrated in vacuo. To the resulting oil was then added methanol $(20 \mathrm{~mL})$, trimethyl orthoformate $(5 \mathrm{~mL}, 46 \mathrm{mmol})$ and $p$-toluesulfonic acid $(0.010 \mathrm{~g}, 0.53 \mathrm{mmol})$ and the mixture was stirred for 4 days. Then $\mathrm{NaHCO}_{3}\left(10 \mathrm{~mL}\right.$, saturated, aqueous) was added along with $\mathrm{CH}_{2} \mathrm{Cl}_{2}(10 \mathrm{~mL})$. The layers were separated and the aqueous layer was extracted with $\mathrm{CH}_{2} \mathrm{Cl}_{2}(5 \times 10 \mathrm{~mL})$. The organic layers were combined, filtered through $\mathrm{Na}_{2} \mathrm{SO}_{4}$, and concentrated in vacuo. The resulting oil was then purified by flash chromatography (98:2 to $97: 3$ hexanes/EtOAc) to afford the product as a colorless oil $(0.3 \mathrm{~g}, 37 \%)$ : ${ }^{1} \mathrm{H}$ NMR $\left(500 \mathrm{MHz}, \mathrm{CDCl}_{3}\right) \delta$ 7.38$7.31(\mathrm{~m}, 4 \mathrm{H}), 7.27-7.22(\mathrm{~m}, 1 \mathrm{H}), 4.64(\mathrm{~s}, 2 \mathrm{H}), 3.19(\mathrm{~s}, 3 \mathrm{H}), 3.17(\mathrm{~s}, 3 \mathrm{H}), 1.93-1.86(\mathrm{~m}$, $4 \mathrm{H}), 1.69-1.59(\mathrm{~m}, 4 \mathrm{H}), 1.04(\mathrm{~s}, 9 \mathrm{H}) ;{ }^{13} \mathrm{C}$ NMR $\left(125 \mathrm{MHz}, \mathrm{CDCl}_{3}\right) \delta$ 140.1, 128.3, 129.3, 126.8, 99.7, 79.4, 65.8, 47.7, 47.6, 38.6, 28.4, 27.3, 25.8; IR (thin film) 2956, 1467, 1256, $1028 \mathrm{~cm}^{-1}$; HRMS (EI) $\mathrm{m} / z$ calcd for $\mathrm{C}_{19} \mathrm{H}_{30} \mathrm{O}_{3} \mathrm{Na}(\mathrm{M}+\mathrm{Na})^{+} 329.2093$, found 329.2094. Anal. Calcd for $\mathrm{C}_{19} \mathrm{H}_{30} \mathrm{O}_{3}$ : C, 74.47; H, 9.87. Found: C, 74.18; H, 9.96.

5. Synthesis of ketal $\mathbf{2 0}$

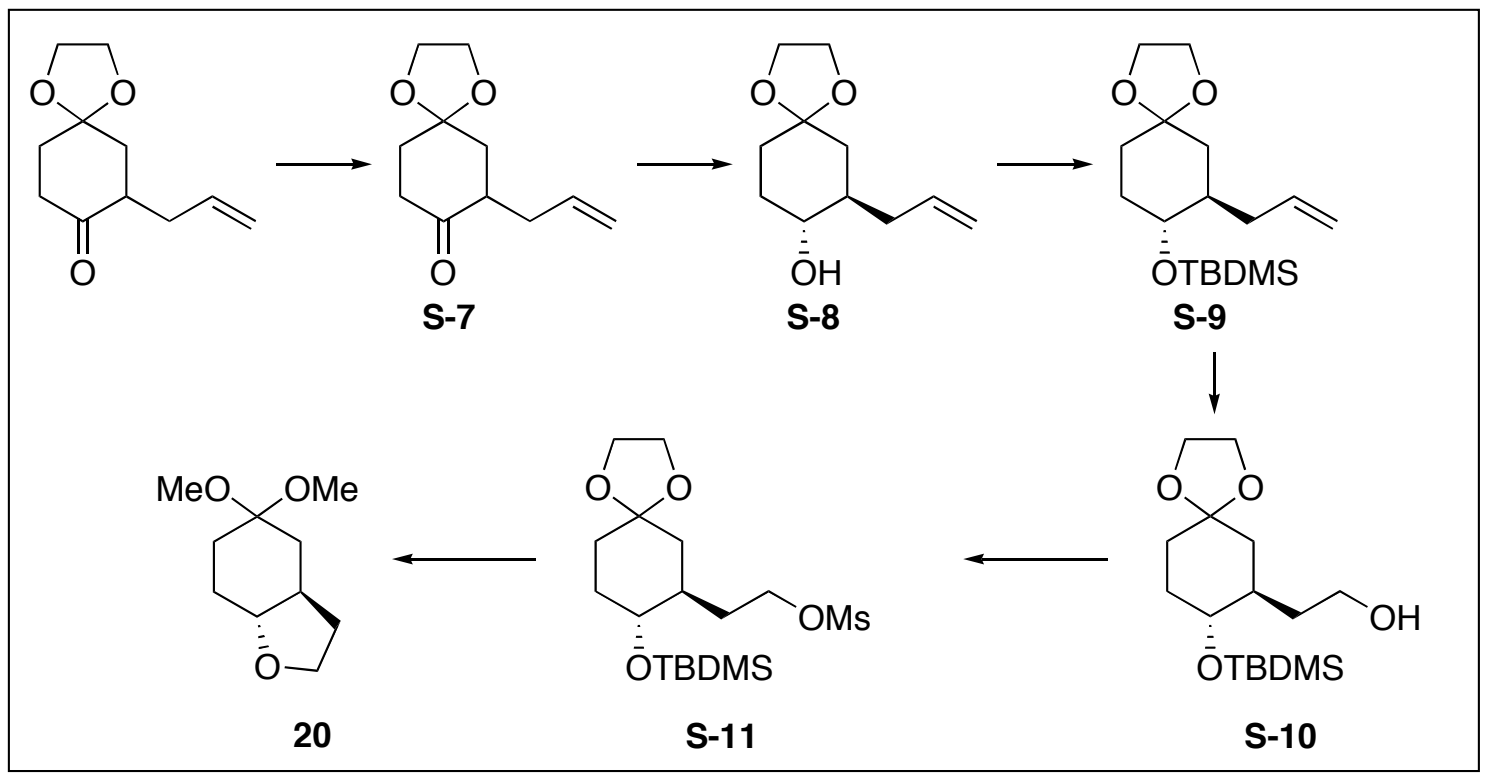




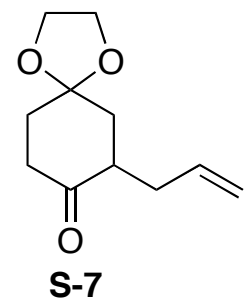

Ketone S-7: To a cooled $\left(-78^{\circ} \mathrm{C}\right)$ solution of 1,4 cyclohexanone ethylene glycol ketal (2.0 g, $13 \mathrm{mmol})$ was added KHMDS $(3.1 \mathrm{~g}, 15 \mathrm{mmol})$ and mixture was stirred for 2 hours. Allyl bromide $(1.7 \mathrm{~mL}, 19 \mathrm{mmol})$ was added and the mixture was warmed (23 $\left.{ }^{\circ} \mathrm{C}\right)$. Then $\mathrm{NaHCO}_{3}\left(40 \mathrm{~mL}\right.$, saturated, aqueous) was added along with $\mathrm{CH}_{2} \mathrm{Cl}_{2}(40 \mathrm{~mL})$. The layers were separated and the aqueous layer was extracted with $\mathrm{CH}_{2} \mathrm{Cl}_{2}(5 \mathrm{x} 40 \mathrm{~mL})$. The organic layers were combined, filtered through $\mathrm{Na}_{2} \mathrm{SO}_{4}$, and concentrated in vacuo. The resulting oil was then purified by flash chromatography (98:2 to 91:9 hexanes/EtOAc) to afford the product as a colorless oil $(0.75 \mathrm{~g}, 30 \%)$ : ${ }^{1} \mathrm{H}$ NMR (500 $\left.\mathrm{MHz} \mathrm{CDCl}_{3}\right) \delta 5.74$ (dddd, $\left.J=16.8,10.2,7.8,6.4,1 \mathrm{H}\right), 5.05-5.01$ (m, 2H), 4.08-3.93 $(\mathrm{m}, 4 \mathrm{H}), 2.74-2.61(\mathrm{~m}, 2 \mathrm{H}), 2.56-2.50(\mathrm{~m}, 1 \mathrm{H}), 2.38(\mathrm{ddd}, J=14.3,5.1,3.2,1 \mathrm{H}), 2.11$ (ddd, $J=13.3,5.8,3.5,1 \mathrm{H}), 2.07-1.93(\mathrm{~m}, 3 \mathrm{H}), 1.69(\mathrm{t}, J=13.2,1 \mathrm{H}) ;{ }^{13} \mathrm{C}$ NMR $(125$

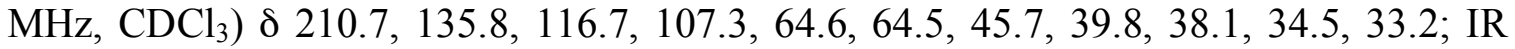
(thin film) 2956, 1715, 1438, $1366 \mathrm{~cm}^{-1}$.

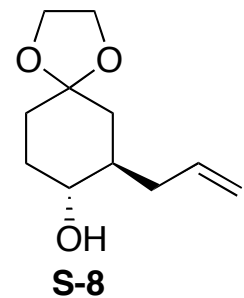

Alcohol S-8: To a cooled $\left(0{ }^{\circ} \mathrm{C}\right)$ solution of S-7 $(0.100 \mathrm{~g}, 0.510 \mathrm{mmol})$ in THF $(1 \mathrm{~mL})$ was added slowly $\mathrm{LiAlH}_{4}(0.030 \mathrm{~g}, 0.81 \mathrm{mmol})$. The mixture was allowed to warm (23 $\left.{ }^{\circ} \mathrm{C}\right)$ and was cooled $\left(0^{\circ} \mathrm{C}\right)$ again. Ethanol $(3 \mathrm{~mL})$ was added slowly along with $\mathrm{CH}_{2} \mathrm{Cl}_{2}(1$ $\mathrm{mL})$. The layers were separated and the aqueous layer was extracted with $\mathrm{CH}_{2} \mathrm{Cl}_{2}(5 \times 1$ $\mathrm{mL}$ ). The organic layers were combined, filtered through $\mathrm{Na}_{2} \mathrm{SO}_{4}$, and concentrated in vacuo. The resulting oil was purified by flash chromatography, affording the product as colorless oil $(0.07 \mathrm{~g}, 74 \%)$; ${ }^{1} \mathrm{H}$ NMR $\left(500 \mathrm{MHz}, \mathrm{CDCl}_{3}\right) \delta 5.81$ (dddd, $J=17.0,10.1$, $7.7,6.7,1 \mathrm{H}), 5.09-5.01(\mathrm{~m}, 2 \mathrm{H}), 4.00-3.90(\mathrm{~m}, 4 \mathrm{H}), 3.33(\mathrm{td}, J=10.1,4.3,1 \mathrm{H}), 2.51-$ $2.42(\mathrm{~m}, 1 \mathrm{H}), 2.02-1.91(\mathrm{~m}, 3 \mathrm{H}), 1.80-1.53(\mathrm{~m}, 5 \mathrm{H}), 1.29(\mathrm{t}, J=12.9,1 \mathrm{H}) ;{ }^{13} \mathrm{C} \mathrm{NMR}$ $\left(125 \mathrm{MHz}, \mathrm{CDCl}_{3}\right) \delta 136.5,116.6,108.4,73.1,64.2,41.6,38.1,36.9,32.9,32.1$; IR (thin film) $3416,2943,2359,1639,1120 \mathrm{~cm}^{-1}$.

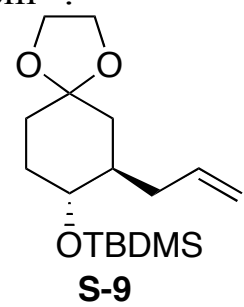


Alkene S-9: To a solution of S-8 $(0.74 \mathrm{~g}, 3.7 \mathrm{mmol})$ and imidazole $(1.3 \mathrm{~g}, 19 \mathrm{mmol})$ in THF $(10 \mathrm{~mL})$ was added TBSCl $(1.1 \mathrm{~g}, 7.5 \mathrm{mmol})$. The mixture was stirred for 3 days, then $\mathrm{NaHCO}_{3}\left(10 \mathrm{~mL}\right.$, saturated, aqueous) was added along with $\mathrm{CH}_{2} \mathrm{Cl}_{2}(10 \mathrm{~mL})$. The layers were separated and the aqueous layer was extracted with $\mathrm{CH}_{2} \mathrm{Cl}_{2}(5 \times 10 \mathrm{~mL})$. The organic layers were combined, filtered through $\mathrm{Na}_{2} \mathrm{SO}_{4}$, and concentrated in vacuo. The resulting oil was then purified by flash chromatography (95:5 hexanes/EtOAc) to afford the product as a colorless oil $(1.2 \mathrm{~g}, 99 \%):{ }^{1} \mathrm{H}$ NMR $\left(500 \mathrm{MHz}, \mathrm{CDCl}_{3}\right) \delta$ 5.77-5.69 (m, $1 \mathrm{H}), 5.03-4.97(\mathrm{~m}, 2 \mathrm{H}), 3.95-3.90(\mathrm{~m}, 4 \mathrm{H}), 3.31(\mathrm{ddd}, J=9.7,9.6,4.1,1 \mathrm{H}), 2.53-2.47$ $(\mathrm{m}, 1 \mathrm{H}), 1.85-1.78(\mathrm{~m}, 3 \mathrm{H}), 1.76-1.50(\mathrm{~m}, 4 \mathrm{H}), 1.25-1.20(\mathrm{~m}, 1 \mathrm{H}), 0.89(\mathrm{~s}, 9 \mathrm{H}), 0.06(\mathrm{~s}$, $6 \mathrm{H}) ;{ }^{13} \mathrm{C}$ NMR $\left(125 \mathrm{MHz}, \mathrm{CDCl}_{3}\right) \delta 136.9,116.2,108.5,73.5,64.3,64.2,41.9,37.6$, 36.7, 32.8, 32.3, 25.8, 18.0, -4.0, -4.7; IR (thin film) 3075, 2952, 1640, 1472, 1379, 1252 $\mathrm{cm}^{-1}$. Anal. Calcd for $\mathrm{C}_{17} \mathrm{H}_{32} \mathrm{O}_{3} \mathrm{Si}$ : C, 65.33; H, 10.32. Found: C, 65.45; H, 10.46.

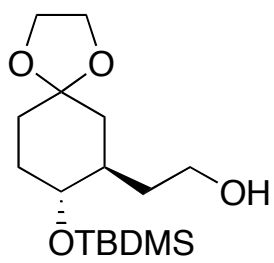

S-10

Alcohol S-10: To a solution of S-9 $(0.2 \mathrm{~g}, 0.6 \mathrm{mmol})$ in acetone: $\mathrm{H}_{2} \mathrm{O}(6 \mathrm{~mL}, 5: 1)$ was added a solution of $\mathrm{OsO}_{4}(4 \%$ wt., $0.04 \mathrm{~mL}, 0.006 \mathrm{mmol})$ and the mixture was stirred for 1 day. A solution of $\mathrm{NaIO}_{4}(0.8 \mathrm{~g}, 4 \mathrm{mmol})$ in $\mathrm{H}_{2} \mathrm{O}(2 \mathrm{~mL})$ was added and the mixture was stirred for 4 hours. Then $\mathrm{CH}_{2} \mathrm{Cl}_{2}(10 \mathrm{~mL})$ was added to the mixture and the layers were separated. The aqueous layer was extracted with $\mathrm{CH}_{2} \mathrm{Cl}_{2}(5 \times 10 \mathrm{~mL})$. The organic layers were combined, filtered through $\mathrm{Na}_{2} \mathrm{SO}_{4}$, and concentrated in vacuo. To the resulting oil was added $\mathrm{CH}_{3} \mathrm{OH}(5 \mathrm{~mL})$ and the mixture was cooled $\left(0{ }^{\circ} \mathrm{C}\right) . \mathrm{NaBH}_{4}(0.03$ g, $0.7 \mathrm{mmol})$ was added and the mixture was warmed $\left(23^{\circ} \mathrm{C}\right)$ and cooled $\left(0{ }^{\circ} \mathrm{C}\right)$ again. Then $\mathrm{NH}_{4} \mathrm{Cl}$ (3 mL, saturated, aqueous) was added slowly along with $\mathrm{CH}_{2} \mathrm{Cl}_{2}(5 \mathrm{~mL})$. The layers were separated and the aqueous layer was extracted with $\mathrm{CH}_{2} \mathrm{Cl}_{2}(5 \times 5 \mathrm{~mL})$. The organic layers were combined, filtered through $\mathrm{Na}_{2} \mathrm{SO}_{4}$, and concentrated in vacuo. The resulting oil was then purified by flash chromatography (83:17 to $75: 25$ hexanes/EtOAc) to afford the product as a colorless oil $(0.180 \mathrm{~g}, 89 \%)$ : ${ }^{1} \mathrm{H}$ NMR (500 $\left.\mathrm{MHz}, \mathrm{CDCl}_{3}\right)$ d 3.98-3.90 (m, 4H), 3.71-3.60 (m, 2H), 3.33 (ddd, $J=9.7,9.5,4.0,1 \mathrm{H}$ ), $1.99(\mathrm{br} \mathrm{s}, 1 \mathrm{H}), 1.94(\mathrm{dtd}, J=13.7,7.5,4.3,1 \mathrm{H}), 1.88-1.81(\mathrm{~m}, 2 \mathrm{H}), 1.79-1.72(\mathrm{~m}, 2 \mathrm{H})$, 1.63 (ddd, $J=12.5,8.1,3.5,1 \mathrm{H}$ ), 1.55 (ddd, $J=16.9,12.0,3.4,1 \mathrm{H}), 1.37-1.30(\mathrm{~m}, 2 \mathrm{H}$ ), 0.89 (s, 9H), $0.07(\mathrm{~m}, 6 \mathrm{H}) ;{ }^{13} \mathrm{C}$ NMR $\left(125 \mathrm{MHz}, \mathrm{CDCl}_{3}\right) \delta$ 108.2, 74.3, 64.3, 64.2, 61.0, 39.4, 38.5, 35.5, 32.5, 32.1, 25.8, 18.0, -4.2, -4.6; IR (thin film) 3432, 2952, 1472, 1252 , $1007 \mathrm{~cm}^{-1}$; HRMS (EI) $m / z$ calcd for $\mathrm{C}_{16} \mathrm{H}_{32} \mathrm{NaO}_{4} \mathrm{Si}(\mathrm{M}+\mathrm{Na})^{+} 339.1967$, found 339.1965. Anal. Calcd for $\mathrm{C}_{16} \mathrm{H}_{32} \mathrm{O}_{4} \mathrm{Si}$ : C, 60.72; H, 10.19. Found: C, 60.56; H, 10.14 . 


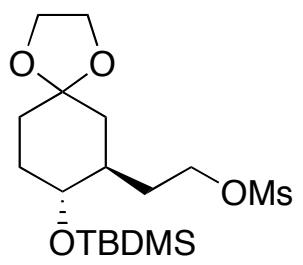

S-11

Mesylate S-11: To a cooled $\left(0^{\circ} \mathrm{C}\right)$ solution of $\mathbf{S - 1 0}(0.17 \mathrm{~g}, 0.50 \mathrm{mmol})$ and methanesulfonyl chloride $(0.05 \mathrm{~mL}, 0.6 \mathrm{mmol})$ in $\mathrm{CH}_{2} \mathrm{Cl}_{2}(2.5 \mathrm{~mL})$ was added pyridine $(0.13 \mathrm{~mL}, 1.6 \mathrm{mmol})$. The mixture was stirred for 1 day and then $\mathrm{NaHCO}_{3}(3 \mathrm{~mL}$ saturated, aqueous) was added. The layers were separated and the aqueous layer was extracted with $\mathrm{CH}_{2} \mathrm{Cl}_{2}(5 \times 3 \mathrm{~mL})$. The organic layers were combined, filtered through $\mathrm{Na}_{2} \mathrm{SO}_{4}$, and concentrated in vacuo. The resulting oil was purified by flash chromatography (83:17 to 75:25 hexanes/EtOAc) to afford the product as a colorless oil (0.21 g, 97\%): ${ }^{1} \mathrm{H}$ NMR (500 MHz, $\left.\mathrm{CDCl}_{3}\right) \delta 4.28(\mathrm{t}, J=6.9,2 \mathrm{H}), 3.97-3.91(\mathrm{~m}, 4 \mathrm{H})$, 3.34 (ddd, $J=9.5,9.3,4.0,1 \mathrm{H}), 3.00(\mathrm{~s}, 3 \mathrm{H}), 2.16(\mathrm{dtd}, J=11.8,7.5,4.4,1 \mathrm{H}), 1.88-1.72$ $(\mathrm{m}, 4 \mathrm{H}), 1.67-1.46(\mathrm{~m}, 3 \mathrm{H}), 1.32(\mathrm{t}, J=12.4,1 \mathrm{H}), 0.89(\mathrm{~s}, 9 \mathrm{H}), 0.07(\mathrm{~s}, 6 \mathrm{H}) ;{ }^{13} \mathrm{C} \mathrm{NMR}$ $\left(125 \mathrm{MHz}, \mathrm{CDCl}_{3}\right) \delta 107.9,74.0,68.5,64.3,64.2,39.0,37.9,37.4,32.5,32.0,31.8,25.8$, $17.9,-4.1,-4.7$; IR (thin film) 2954, 1473, 1358, 1252, $1176 \mathrm{~cm}^{-1}$.

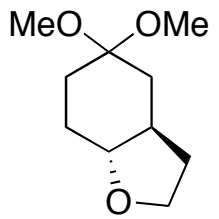

20

Ketal 20: To a solution of S-11 $(1.0 \mathrm{~g}, 2.6 \mathrm{mmol})$ in THF $(12 \mathrm{~mL})$ was added a solution of TBAF ( $1 \mathrm{M}$ in THF, $12 \mathrm{~mL}, 12 \mathrm{mmol})$ and the mixture stirred for 1 day. $\mathrm{H}_{2} \mathrm{O}(10 \mathrm{~mL})$ was added along with along with $\mathrm{CH}_{2} \mathrm{Cl}_{2}(10 \mathrm{~mL})$. The layers were separated and the aqueous layer was extracted with $\mathrm{CH}_{2} \mathrm{Cl}_{2}(5 \times 10 \mathrm{~mL})$. The organic layers were combined, filtered through $\mathrm{Na}_{2} \mathrm{SO}_{4}$, and concentrated in vacuo. The resulting oil was then purified by flash chromatography (83:17 hexanes/EtOAc) to afford the product as a colorless oil contaminated with TBAF. The product was used for the next step without further purification. To the product was added methanol $(20 \mathrm{~mL})$, trimethyl orthoformate ( $5 \mathrm{~mL}, 60 \mathrm{mmol}$ ) and 1 drop of $\mathrm{H}_{2} \mathrm{SO}_{4}$ (concentrated). The mixture was stirred for 1 day, then $\mathrm{NaHCO}_{3}\left(10 \mathrm{~mL}\right.$, saturated, aqueous) was added along with $\mathrm{CH}_{2} \mathrm{Cl}_{2}(10 \mathrm{~mL})$. The layers were separated and the aqueous layer was extracted with $\mathrm{CH}_{2} \mathrm{Cl}_{2}(5 \times 10 \mathrm{~mL})$. The organic layers were combined, filtered through $\mathrm{Na}_{2} \mathrm{SO}_{4}$, and concentrated in vacuo. The resulting oil was then purified by flash chromatography (99:1 to 83:17 hexanes/EtOAc) to afford the product as a colorless oil $(0.26 \mathrm{~g}, 55 \%)$ : ${ }^{1} \mathrm{H} \mathrm{NMR}\left(500 \mathrm{MHz}, \mathrm{CDCl}_{3}\right) \delta$ $3.99-3.92(\mathrm{~m}, 2 \mathrm{H}), 3.22(\mathrm{~s}, 3 \mathrm{H}), 3.18(\mathrm{~s}, 3 \mathrm{H}), 3.11(\mathrm{ddd}, J=11.1,10.1,3.9,1 \mathrm{H}), 2.29$ $(\mathrm{dt}, J=13.0,3.2,1 \mathrm{H}), 2.14(\mathrm{ddd}, J=13.8,6.5,3.2,1 \mathrm{H}), 1.99-1.94(\mathrm{~m}, 2 \mathrm{H}), 1.68$ (dddd, $J$ $=22.2,12.7,6.5,3.3,1 \mathrm{H}), 1.60-1.46(\mathrm{~m}, 2 \mathrm{H}), 1.39(\mathrm{td}, J=13.7,3.9,1 \mathrm{H}), 1.21(\mathrm{t}, J=$ $12.8,1 \mathrm{H}) ;{ }^{13} \mathrm{C} \mathrm{NMR}\left(125 \mathrm{MHz}, \mathrm{CDCl}_{3}\right) \delta 100.9,82.9,67.9,48.1,47.4,41.4,35.0,30.7$, 30.4, 26.7; IR (thin film) 2952, 1454, 1358, $1244 \mathrm{~cm}^{-1}$. 


\section{Reactions Featured in Corresponding Article}

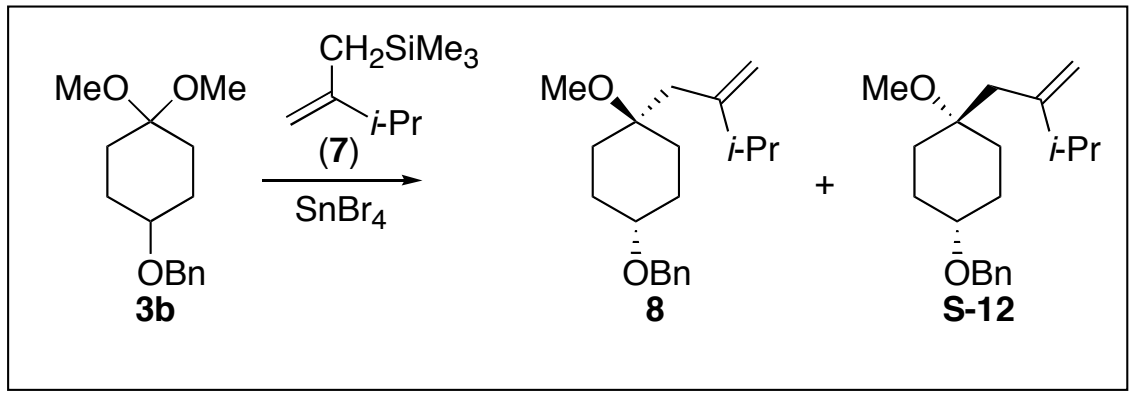

Benzyl Ethers 8 \& S-12: To a cooled $\left(-78^{\circ} \mathrm{C}\right)$ solution of $3 \mathbf{b}(0.15 \mathrm{~g}, 0.60 \mathrm{mmol})$ in $\mathrm{CH}_{2} \mathrm{Cl}_{2}(3 \mathrm{~mL})$ was added silane $7^{5}(0.19 \mathrm{~g}, 1.2 \mathrm{mmol})$ and the mixture was stirred for 2 minutes, then $\mathrm{SnBr}_{4}\left(1 \mathrm{M}\right.$ in $\left.\mathrm{CH}_{2} \mathrm{Cl}_{2}, 0.72 \mathrm{~mL}, 0.72 \mathrm{mmol}\right)$ was added. The solution was allowed to warm $\left(0{ }^{\circ} \mathrm{C}\right)$ and $\mathrm{NaHCO}_{3}(3 \mathrm{~mL}$ saturated, aqueous $)$ was added. The layers were separated and the aqueous layer was extracted with $\mathrm{CH}_{2} \mathrm{Cl}_{2}(5 \times 3 \mathrm{~mL})$. The organic layers were combined, filtered through $\mathrm{Na}_{2} \mathrm{SO}_{4}$, and concentrated in vacuo. A selectivity of 94:6 (8 : $\mathbf{S - 1 2}$ ) was determined by subjecting the unpurified oil to GCMS: $t_{R}$ (major) $18.7 \mathrm{~min}, t_{R}$ (minor) $19.4 \mathrm{~min}$. The resulting oil was purified by flash chromatography (98:2 to $97: 3$ hexanes/EtOAc) to afford the product as a colorless oil (0.15 g, 80\%): ${ }^{1} \mathrm{H}$ NMR (500 MHz, $\left.\mathrm{CDCl}_{3}\right) \quad \delta$ 7.35-7.32 (m, 4H), 7.28-7.23 (m, $\left.1 \mathrm{H}\right)$, 4.90-4.89 (m, 1H), 4.81-4.80 (m, 1H), $4.50(\mathrm{~s}, 2 \mathrm{H}), 3.62-3.56(\mathrm{~m}, 1 \mathrm{H}), 3.20(\mathrm{~s}, 3 \mathrm{H}), 2.28$ (sep, $J=6.8,1 \mathrm{H}), 2.21(\mathrm{~s}, 2 \mathrm{H}), 1.73-1.64(\mathrm{~m}, 6 \mathrm{H}), 1.58-1.54(\mathrm{~m}, 2 \mathrm{H}), 1.03(\mathrm{~d}, J=6.8$, $6 \mathrm{H}) ;{ }^{13} \mathrm{C}$ NMR $\left(125 \mathrm{MHz}, \mathrm{CDCl}_{3}\right), \delta 152.1,139.3,128.3,127.34,127.28,110.0,75.6$, 73.3, 69.7, 48.5, 40.1, 34.1, 29.0, 25.9, 22.0; IR (thin film) 3085, 3030, 2958, 2870, 1637, $1455 \mathrm{~cm}^{-1}$; HRMS (EI) $\mathrm{m} / \mathrm{z}$ calcd for $\mathrm{C}_{20} \mathrm{H}_{30} \mathrm{O}_{2} \mathrm{Na}(\mathrm{M}+\mathrm{Na})^{+} 325.2144$, found 325.2139 . Anal. Calcd for $\mathrm{C}_{20} \mathrm{H}_{30} \mathrm{O}_{2}$ : C, 79.42; H, 10.00. Found: C, 79.01; H, 10.22.

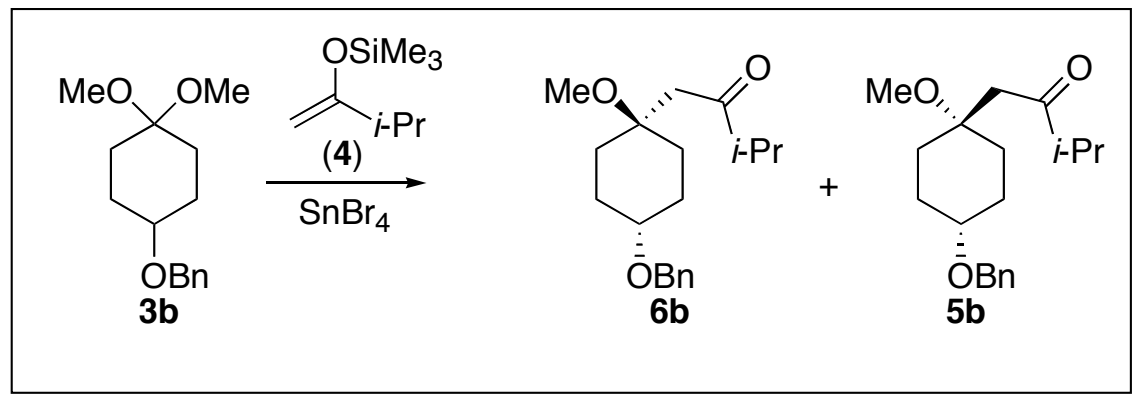

Benzyl Ethers 5b \& 6b: To a cooled $\left(-78^{\circ} \mathrm{C}\right)$ solution of $\mathbf{3 b}(0.050 \mathrm{~g}, 0.200 \mathrm{mmol})$ in toluene $(1 \mathrm{~mL})$ was added enol ether $4^{6}(0.076 \mathrm{~g}, 0.24 \mathrm{mmol})$ and the was mixture stirred for 2 minutes, then $\mathrm{SnBr}_{4}\left(1 \mathrm{M}\right.$ in $\left.\mathrm{CH}_{2} \mathrm{Cl}_{2}, 0.24 \mathrm{~mL}, 0.24 \mathrm{mmol}\right)$ was added. The solution was allowed to warm $\left(0{ }^{\circ} \mathrm{C}\right)$ and $\mathrm{NaHCO}_{3}(3 \mathrm{~mL}$ saturated, aqueous $)$ was added. The layers were separated and the aqueous layer was extracted with $\mathrm{CH}_{2} \mathrm{Cl}_{2}(5 \times 3 \mathrm{~mL})$. The organic layers were combined, filtered through $\mathrm{Na}_{2} \mathrm{SO}_{4}$, and concentrated in vacuo. A selectivity of 94:6 (6b : 5b) was determined by subjecting the unpurified oil to GCMS: $t_{R}$ (major) $19.9 \mathrm{~min}, \mathrm{t}_{\mathrm{R}}$ (minor) $20.4 \mathrm{~min}$. The resulting oil was purified by flash 
chromatography (97:3 to $96: 4$ hexanes/EtOAc) to afford the product as a colorless oil (0.05 g, 86\%): ${ }^{1} \mathrm{H}$ NMR (500 MHz, $\left.\mathrm{CDCl}_{3}\right) \delta$ 7.34-7.32 (m, 4H), 7.28-7.25 (m, 1H), 4.50 $(\mathrm{s}, 2 \mathrm{H}), 3.61-3.58(\mathrm{~m}, 1 \mathrm{H}), 3.23(\mathrm{~s}, 3 \mathrm{H}), 2.70(\mathrm{sep}, J=6.9,1 \mathrm{H}), 2.62(\mathrm{~s}, 2 \mathrm{H}), 1.85-1.69$ $(\mathrm{m}, 6 \mathrm{H}), 1.64-1.61(\mathrm{~m}, 2 \mathrm{H}), 1.07(\mathrm{~d}, J=6.9,6 \mathrm{H}) ;{ }^{13} \mathrm{C} \mathrm{NMR}\left(125 \mathrm{MHz}, \mathrm{CDCl}_{3}\right) \delta 213.1$, 139.1, 127.3, 127.3, 75.2, 72.7, 69.7, 49.0, 46.5, 42.1, 29.1, 25.6, 18.0; IR (thin film) 2925, 1705, 1456, 1368, $1062 \mathrm{~cm}^{-1}$; HRMS (ES) $m / z$ calcd for $\mathrm{C}_{19} \mathrm{H}_{29} \mathrm{O}_{3}(\mathrm{M}+\mathrm{H})^{+}$ 305.2117, found 305.2102. Anal. Calcd for $\mathrm{C}_{19} \mathrm{H}_{28} \mathrm{O}_{3}$ : C, 74.96; H, 9.27. Found: $\mathrm{C}$, $74.80 ; \mathrm{H}, 9.15$.

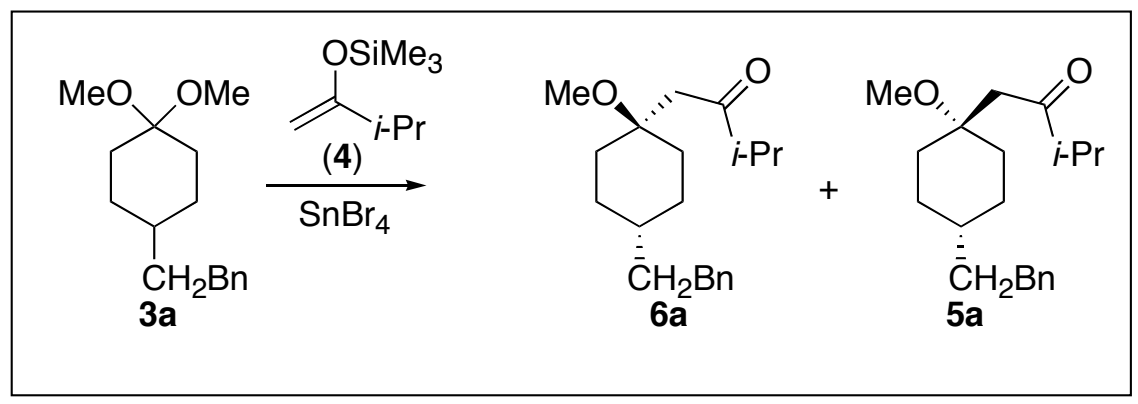

Ketones 5a \& 6a: To a cooled $\left(-78^{\circ} \mathrm{C}\right)$ solution of 3a $(0.100 \mathrm{~g}, 0.402 \mathrm{mmol})$ in toluene $(1 \mathrm{~mL})$ was added enol ether $4(0.08 \mathrm{~g}, 0.5 \mathrm{mmol})$ and the mixture was allowed to stir for 2 minutes, then a solution of $\mathrm{SnBr}_{4}\left(1 \mathrm{M}\right.$ in $\left.\mathrm{CH}_{2} \mathrm{Cl}_{2}, 0.48 \mathrm{~mL}, 0.48 \mathrm{mmol}\right)$ was added. The solution was allowed to warm $\left(0{ }^{\circ} \mathrm{C}\right)$ and $\mathrm{NaHCO}_{3}(1 \mathrm{~mL}$ saturated, aqueous) was added. The layers were separated and the aqueous layer was extracted with $\mathrm{CH}_{2} \mathrm{Cl}_{2}(5 \mathrm{x}$ $1 \mathrm{~mL})$. The organic layers were combined, filtered through $\mathrm{Na}_{2} \mathrm{SO}_{4}$, and concentrated in vacuo. A selectivity of 4:96 (6a : 5a) was determined by subjecting the unpurified oil to GCMS: $t_{R}$ (major) 19.9 min, $t_{R}$ (minor) $19.8 \mathrm{~min}$. The resulting oil was purified by flash chromatography (95:5 to $91: 9$ hexanes/EtOAc) to afford the product as a colorless oil (0.087 g, 71\%): ${ }^{1} \mathrm{H}$ NMR (500 MHz, $\left.\mathrm{CDCl}_{3}\right) \delta$ 7.31-7.25 (m, 2H), 7.21-7.14 (m, 3H), $3.21(\mathrm{~s}, 3 \mathrm{H}), 2.67(\mathrm{sep}, J=6.9,1 \mathrm{H}), 2.62-2.61(\mathrm{~m}, 2 \mathrm{H}), 2.57(\mathrm{~s}, 2 \mathrm{H}), 1.83-1.81(\mathrm{~m}, 2 \mathrm{H})$, $1.57-1.51(\mathrm{~m}, 4 \mathrm{H}), 1.44$ (ddd, $J=13.6,13.4,3.1,2 \mathrm{H}), 1.26-1.23(\mathrm{~m}, 3 \mathrm{H}), 1.06(\mathrm{~d}, J=6.9$, $6 \mathrm{H}) ;{ }^{13} \mathrm{C}$ NMR $\left(125 \mathrm{MHz}, \mathrm{CDCl}_{3}\right), \delta$ 213.4, 143.0, 128.3, 128.2, 125.5, 75.0, 48.6, 47.5, 42.2, 38.8, 36.2, 33.6, 33.2, 27.8, 18.0; HRMS (EI) $m / z$ calcd for $\mathrm{C}_{20} \mathrm{H}_{30} \mathrm{O}_{2} \mathrm{Na}(\mathrm{M}+\mathrm{Na})^{+}$ 325.2144, found 325.2140. Anal. Calcd for $\mathrm{C}_{20} \mathrm{H}_{30} \mathrm{O}_{2}: \mathrm{C}, 79.42 ; \mathrm{H}, 10.00$. Found: $\mathrm{C}$, 79.58; H, 10.03 .

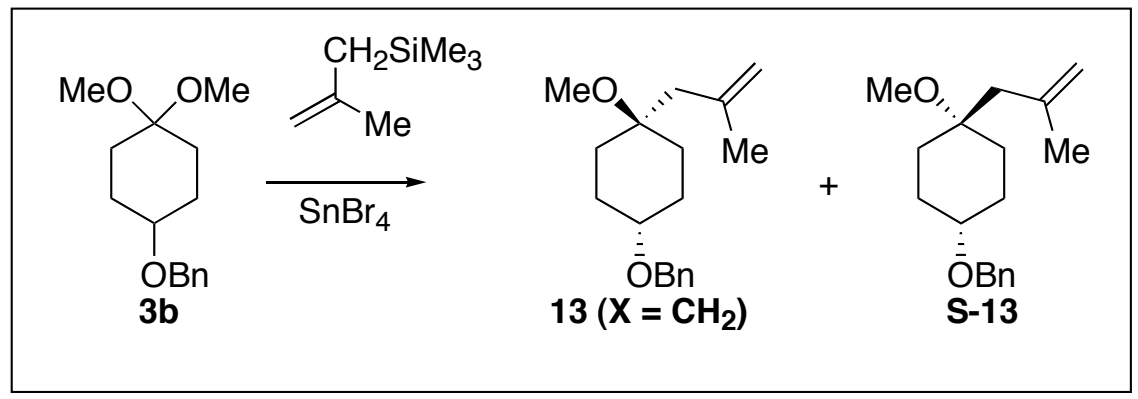

Benzyl Ethers 13 \& S-13: To a cooled $\left(-78^{\circ} \mathrm{C}\right)$ solution of $\mathbf{3 b}(0.150 \mathrm{~g}, 0.600 \mathrm{mmol})$ in $\mathrm{CH}_{2} \mathrm{Cl}_{2}(3 \mathrm{~mL})$ was added 2-methyl-allyltrimethylsilane $(0.31 \mathrm{~mL}, 2.4 \mathrm{mmol})$ and 
allowed to stir for 2 minutes, then $\mathrm{SnBr}_{4}\left(1 \mathrm{M}\right.$ in $\left.\mathrm{CH}_{2} \mathrm{Cl}_{2}, 0.72 \mathrm{~mL}, 0.72 \mathrm{mmol}\right)$ was added. The solution was allowed to warm $\left(0^{\circ} \mathrm{C}\right)$ and $\mathrm{NaHCO}_{3}(3 \mathrm{~mL}$ saturated, aqueous $)$ was added. The layers were separated and the aqueous layer was extracted with $\mathrm{CH}_{2} \mathrm{Cl}_{2}$ $(5 \times 3 \mathrm{~mL})$. The organic layers were combined, filtered through $\mathrm{Na}_{2} \mathrm{SO}_{4}$, and concentrated in vacuo. A selectivity of 88:12 (13: S-13) was determined by subjecting the unpurified oil to $\mathrm{GC}: \mathrm{t}_{\mathrm{R}}$ (major) $16.3 \mathrm{~min}, \mathrm{t}_{\mathrm{R}}$ (minor) $17.0 \mathrm{~min}$. The resulting oil was purified by flash chromatography (98:2 to $96: 4$ hexanes/EtOAc) to afford the products (13, S-13) separately as colorless oils. $13(0.099 \mathrm{~g}, 60 \%):{ }^{1} \mathrm{H}$ NMR $\left(500 \mathrm{MHz}, \mathrm{CDCl}_{3}\right) \delta$ 7.34-7.33 (m, 4H), 7.27-7.23 (m, 1H), 4.86-4.85 (m, 1H), $4.70(\mathrm{~s}, 1 \mathrm{H}), 4.50(\mathrm{~s}, 2 \mathrm{H}), 3.61-$ $3.56(\mathrm{~m}, 1 \mathrm{H}), 3.18(\mathrm{~s}, 3 \mathrm{H}), 2.20(\mathrm{~s}, 2 \mathrm{H}), 1.81(\mathrm{~s}, 3 \mathrm{H}), 1.76-1.64(\mathrm{~m}, 6 \mathrm{H}), 1.58-1.55(\mathrm{~m}$, $2 \mathrm{H}) ;{ }^{13} \mathrm{C}$ NMR $\left(125 \mathrm{MHz}, \mathrm{CDCl}_{3}\right) \delta 142.2,139.2,128.3,127.3,127.2,114.1,75.3,73.4$, 69.7, 48.5, 42.7, 29.2, 26.0, 24.2; IR (thin film) 3063, 2930, 1643, 1491,1367 $\mathrm{cm}^{-1}$; HRMS (EI) $m / z$ calcd for $\mathrm{C}_{17} \mathrm{H}_{22} \mathrm{O}(\mathrm{M}-\mathrm{MeOH})^{+} 242.1671$, found 242.1668. Anal. Calcd for $\mathrm{C}_{18} \mathrm{H}_{26} \mathrm{O}_{2}$ : C, 78.79; H, 9.55. Found: C, 78.52; H, 9.68. S-13 (0.017 g, 10\%): ${ }^{1} \mathrm{H}$ NMR $\left(500 \mathrm{MHz}, \mathrm{CDCl}_{3}\right), \delta$ 7.36-7.31 (m, 4H) 7.27-7.24 (m, 1H), $4.84(\mathrm{dt}, J=3.9$, $1.5 \mathrm{~Hz}, 1 \mathrm{H}), 4.66(\mathrm{~m}, 1 \mathrm{H}), 4.56(\mathrm{~s}, 2 \mathrm{H}), 3.31(\mathrm{tt}, J=10.4,4.0,1 \mathrm{H}), 3.19(\mathrm{~s}, 3 \mathrm{H}), 2.14(\mathrm{~s}$, $2 \mathrm{H}), 1.89-1.79(\mathrm{~m}, 7 \mathrm{H}) 1.65-1.58(\mathrm{~m}, 2 \mathrm{H}), 1.22(\mathrm{ddd}, J=13.7,13.4,3.7,2 \mathrm{H}) ;{ }^{13} \mathrm{C} \mathrm{NMR}$ $\left(125 \mathrm{MHz}, \mathrm{CDCl}_{3}\right) \delta 142.4,139.2,128.3,127.5,127.3,114.2,76.8,74.4,69.8,48.1$, 43.4, 32.1, 27.3, 24.3; IR (thin film) 2934, 1637, 1457, 1367, $1076 \mathrm{~cm}^{-1}$; HRMS (EI) $\mathrm{m} /$ $z$ calcd for $\mathrm{C}_{14} \mathrm{H}_{19} \mathrm{O}_{2}\left(\mathrm{M}-\mathrm{C}_{4} \mathrm{H}_{7}\right)^{+}$201.1279, found 201.1276. Anal. Calcd for $\mathrm{C}_{18} \mathrm{H}_{26} \mathrm{O}_{2}$ : C, 78.79; H, 9.55. Found: C, 78.57; H, 9.72.

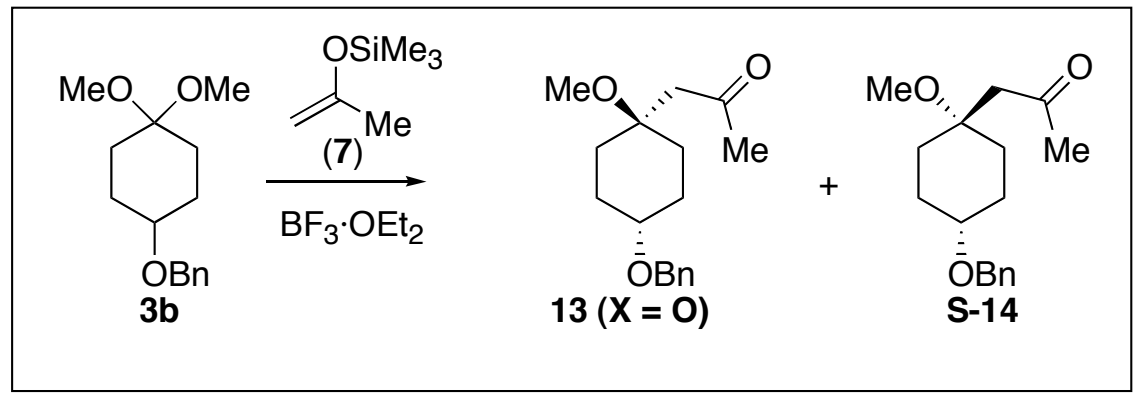

Ketones 13 \& S-14: To a cooled $\left(-78^{\circ} \mathrm{C}\right)$ solution of $\mathbf{3 b}(0.050 \mathrm{~g}, 0.20 \mathrm{mmol})$ in $\mathrm{CH}_{2} \mathrm{Cl}_{2}$ $(1 \mathrm{~mL})$ was added 2-(trimethylsiloxy propene) $(0.08 \mathrm{~g}, 0.24 \mathrm{mmol})$ and the mixture was stirred for 2 minutes, then $\mathrm{BF}_{3} \bullet \mathrm{OEt}_{2}(0.21 \mathrm{~mL}, 0.24 \mathrm{mmol})$ was added. The solution was allowed to warm to $\left(0{ }^{\circ} \mathrm{C}\right)$ and $\mathrm{NaHCO}_{3}(3 \mathrm{~mL}$ saturated, aqueous) was added. The layers were separated and the aqueous layer was extracted with $\mathrm{CH}_{2} \mathrm{Cl}_{2}(5 \times 3 \mathrm{~mL})$. The organic layers were combined, filtered through $\mathrm{Na}_{2} \mathrm{SO}_{4}$, and concentrated in vacuo. A selectivity of 61:49 (13 : S-14) was determined by subjecting the unpurified oil to ${ }^{1} \mathrm{H}$ NMR. The resulting oil was purified by flash chromatography (91:9 to 83:17 hexanes/EtOAc) to afford the product $\mathbf{1 3}$ and a mixture of $\mathbf{1 3} \& \mathbf{S}-\mathbf{1 4}$ (as a 8:92 mixture as determined by NMR) as colorless oils. $13(0.023 \mathrm{~g}, 42 \%)$ : ${ }^{1} \mathrm{H}$ NMR (500 $\mathrm{MHz}$, $\left.\mathrm{CDCl}_{3}\right) \delta$ 7.35-7.34 (m, 4H), 7.29-7.25 (m, 1H), $4.50(\mathrm{~s}, 2 \mathrm{H}), 3.62-3.59(\mathrm{~m}, 1 \mathrm{H}), 3.26(\mathrm{~s}$, $3 \mathrm{H}), 2.59(\mathrm{~s}, 2 \mathrm{H}), 2.20(\mathrm{~s}, 3 \mathrm{H}), 1.81-1.69(\mathrm{~m}, 6 \mathrm{H}), 1.65-1.62(\mathrm{~m}, 2 \mathrm{H}) ;{ }^{13} \mathrm{C} \mathrm{NMR}(125$ $\left.\mathrm{MHz}_{2} \mathrm{CDCl}_{3}\right) \delta$ 207.6, 139.1, 128.3, 127.33, 127.32, 75.0, 72.8, 69.8, 49.6, 49.0, 32.2, 29.2, 25.7; IR (thin film) 3067, 3023, 1705, 1433, 1422, $1068 \mathrm{~cm}^{-1}$; HRMS (EI) $\mathrm{m} / \mathrm{z}$ calcd for $\mathrm{C}_{16} \mathrm{H}_{20} \mathrm{O}_{2}(\mathrm{M}-\mathrm{MeOH})^{+}$244.1463, found 244.1475. Anal. Calcd for $\mathrm{C}_{17} \mathrm{H}_{24} \mathrm{O}_{3}$ : 
C, 73.88; H, 8.75. Found: C, 74.12; H, 8.97. 13 \& S-14 (0.021 g, 38\%). ${ }^{1} \mathrm{H}$ NMR (500 $\left.\mathrm{MHz}, \mathrm{CDCl}_{3}\right)$ d 7.35-7.30 (m, 4.29H), 7.28-7.23 (m, 1.7H), $4.55(\mathrm{~s}, 2 \mathrm{H}), 3.62-3.59(\mathrm{~m}$, $0.1 \mathrm{H}), 3.35$ (ddt, $J=10.2,8.3,4.0,1 \mathrm{H}), 3.25(\mathrm{~s}, 0.24 \mathrm{H}), 3.24(\mathrm{~s}, 3 \mathrm{H}) 2.58(\mathrm{~s}, 0.17 \mathrm{H})$, $2.54(\mathrm{~s}, 2 \mathrm{H}), 2.20(\mathrm{~s}, 0.23 \mathrm{H}), 2.19(\mathrm{~s}, 3 \mathrm{H}), 1.90-1.68(\mathrm{~m}, 4.87 \mathrm{H}), 1.75-1.66(\mathrm{~m}, 0.63 \mathrm{H})$ $1.67-1.59(\mathrm{~m}, 2.61 \mathrm{H}), 1.41(\mathrm{ddd}, J=13.5,13.4,3.8,2 \mathrm{H}) ;{ }^{13} \mathrm{C} \mathrm{NMR}\left(125 \mathrm{MHz}, \mathrm{CDCl}_{3}\right) \delta$ 207.7, 139.0, 128.31, 127.5, 127.4, 76.1, 74.2, 69.9, 49.5, 48.6, 32.2, 31.8, 27.1; IR (thin film) 2933, 2844, 1706, 1450, $1228 \mathrm{~cm}^{-1}$; HRMS (EI) $\mathrm{m} / \mathrm{z}$ calcd for $\mathrm{C}_{13} \mathrm{H}_{21} \mathrm{O}_{2}$ (M$\mathrm{MeOH})^{+}$244.1463, found 244.1461. Anal. Calcd for $\mathrm{C}_{17} \mathrm{H}_{24} \mathrm{O}_{3}: \mathrm{C}, 73.88 ; \mathrm{H}, 8.75$. Found: C, 73.74; H, 8.83.

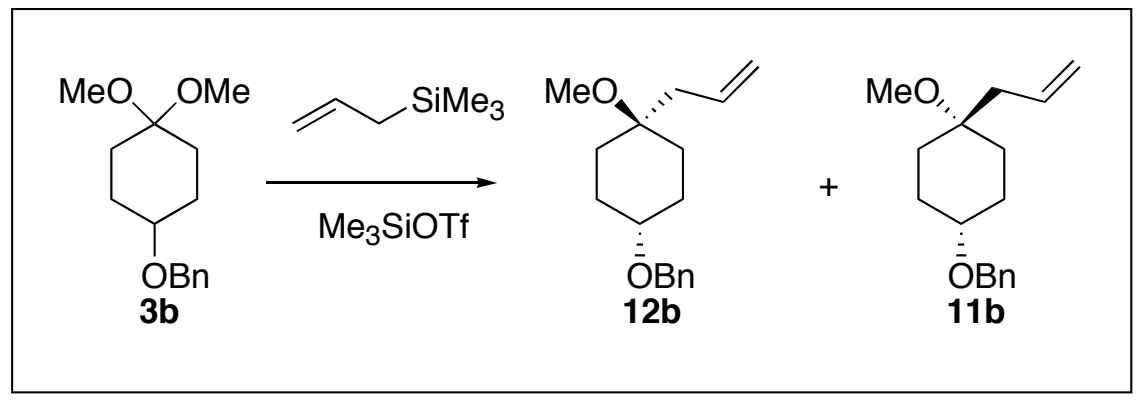

Benzyl Ethers 11b \& 12b: To a cooled $\left(-78^{\circ} \mathrm{C}\right)$ solution of $\mathbf{3 b}(0.150 \mathrm{~g}, 0.600 \mathrm{mmol})$ in $\mathrm{CH}_{2} \mathrm{Cl}_{2}(3 \mathrm{~mL})$ was added allyltrimethylsilane $(0.39 \mathrm{~mL}, 2.4 \mathrm{mmol})$ and the mixture was stirred for 2 minutes, then TMSOTf $(0.13 \mathrm{~mL}, 0.72 \mathrm{mmol})$ was added. The solution was warmed $\left(0{ }^{\circ} \mathrm{C}\right)$ and $\mathrm{NaHCO}_{3}(3 \mathrm{~mL}$ saturated, aqueous $)$ was added. The layers were separated and the aqueous layer was extracted with $\mathrm{CH}_{2} \mathrm{Cl}_{2}(5 \times 3 \mathrm{~mL})$. The organic layers were combined, filtered through $\mathrm{Na}_{2} \mathrm{SO}_{4}$, and concentrated in vacuo. A selectivity of 80:20 (12b : 11b) was determined by subjecting the unpurified oil to GC: $t_{R}$ (major) $15.4 \mathrm{~min}, \mathrm{t}_{\mathrm{R}}$ (minor) $16.0 \mathrm{~min}$. The resulting oil was purified by flash chromatography (97:3 to $96: 4$ hexanes/EtOAc) to afford the products (s-1, s-2) separately as colorless oils. 12b $(0.104 \mathrm{~g}, 67 \%):{ }^{1} \mathrm{H}$ NMR $\left(500 \mathrm{MHz}, \mathrm{CDCl}_{3}\right) \quad \delta \quad 7.38-7.31(\mathrm{~m}, 4 \mathrm{H}), 7.28-7.24(\mathrm{~m}$, $1 \mathrm{H}), 5.82(\mathrm{ddt}, J=17.4,10.2,7.2,1 \mathrm{H}), 5.10-5.03(\mathrm{~m}, 2 \mathrm{H}), 4.50(\mathrm{~s}, 2 \mathrm{H}), 3.61-3.57(\mathrm{~m}$, $1 \mathrm{H}), 3.18(\mathrm{~s}, 3 \mathrm{H}) 2.26(\mathrm{~d}, J=7.2,2 \mathrm{H}), 1.77-1.64(\mathrm{~m}, 6 \mathrm{H}), 1.56(\mathrm{dt}, J=13.2,4.0,2 \mathrm{H})$; ${ }^{13} \mathrm{C}$ NMR $\left(125 \mathrm{MHz}, \mathrm{CDCl}_{3}\right) \delta 139.2,133.7,128.3,127.30,127.25,117.3,74.7,73.4$, 69.7, 48.4, 40.3, 28.8, 25.8; IR (thin film) 2931, 1454, 1369, $1075 \mathrm{~cm}^{-1}$; HRMS (EI) $\mathrm{m} /$ $\mathrm{z}$ calcd for $\mathrm{C}_{14} \mathrm{H}_{19} \mathrm{O}_{2}\left(\mathrm{M}_{-} \mathrm{C}_{3} \mathrm{H}_{5}\right)^{+}$219.1385, found 219.1383. Anal. Calcd for $\mathrm{C}_{17} \mathrm{H}_{24} \mathrm{O}_{2}$ : C, 78.42; H, 9.29. Found: C, 78.67; H, 9.56. 11b $\left(0.028\right.$ g, 18\%) ${ }^{1} \mathrm{H}$ NMR $(500 \mathrm{MHz}$, $\left.\mathrm{CDCl}_{3}\right) \delta$ 7.36-7.32 (m, 4H), 7.28-7.25 (m, 1H), $5.80(\mathrm{ddt}, J=17.5,10.3,7.3,1 \mathrm{H}), 5.08-$ $5.02(\mathrm{~m}, 2 \mathrm{H}), 4.56(\mathrm{~s}, 2 \mathrm{H}), 3.32(\mathrm{tt}, J=10.4,4.0,1 \mathrm{H}), 3.17(\mathrm{~s}, 3 \mathrm{H}) 2.20-2.18(\mathrm{~m}, 2 \mathrm{H})$, 1.88-1.81 (m, 4H), 1.65-1.58 (m, 2H), 1.25-1.19 (m, 2H); ${ }^{13} \mathrm{C}$ NMR $\left(125 \mathrm{MHz}, \mathrm{CDCl}_{3}\right)$ $\delta$ 139.1, 133.8, 128.3, 127.5, 127.3, 117.5, 73.9, 69.8, 48.2, 40.9, 34.3, 31.6, 27.2; IR (thin film) 2941, 1449, 1364, $1070 \mathrm{~cm}^{-1}$. Anal. Calcd for $\mathrm{C}_{17} \mathrm{H}_{24} \mathrm{O}_{2}$ : C, 78.42; H, 9.29. Found: $\mathrm{C}, 78.31 ; \mathrm{H}, 9.44$. 


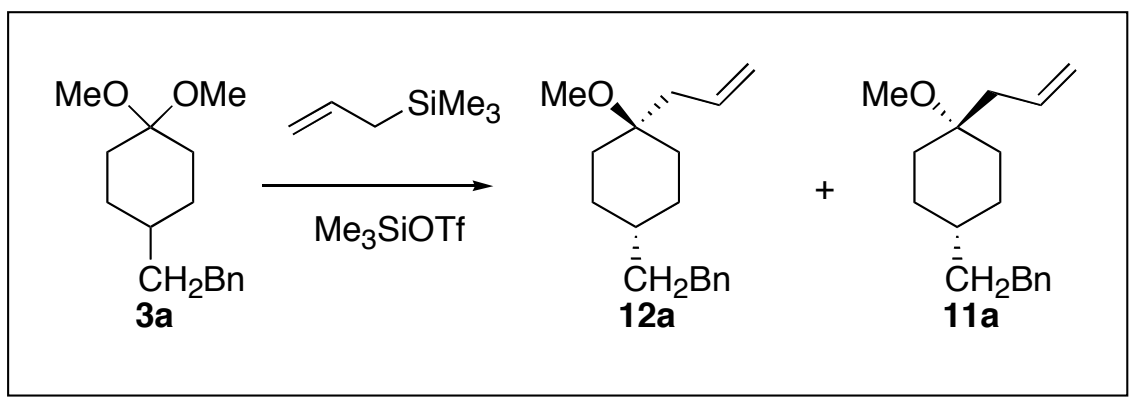

Alkenes 11a \& 12a: To a cooled $\left(-78{ }^{\circ} \mathrm{C}\right)$ solution of 3a $(0.100 \mathrm{~g}, 0.402 \mathrm{mmol})$ in $\mathrm{CH}_{2} \mathrm{Cl}_{2}(2 \mathrm{~mL})$ was added allyltrimethylsilane $(0.26 \mathrm{~mL}, 1.6 \mathrm{mmol})$ and the mixture was allowed to stir for 2 minutes, then TMSOTf $(0.09 \mathrm{~mL}, 0.50 \mathrm{mmol})$ was added. The solution was allowed to warm $\left(0{ }^{\circ} \mathrm{C}\right)$ and $\mathrm{NaHCO}_{3}(3 \mathrm{~mL}$ saturated, aqueous $)$ was added. The layers were separated and the aqueous layer was extracted with $\mathrm{CH}_{2} \mathrm{Cl}_{2}(5 \times 2 \mathrm{~mL})$. The organic layers were combined, filtered through $\mathrm{Na}_{2} \mathrm{SO}_{4}$, and concentrated in vacuo. A selectivity of 5:95 (12a : 11a) was determined by subjecting the unpurified oil to GCMS: $t_{R}$ (major) $17.2 \mathrm{~min}, t_{R}$ (minor) $17.4 \mathrm{~min}$. The resulting oil was purified by flash chromatography (99:1 to 98:2 hexanes/EtOAc) to afford the product (as a 95:5 mixture as determined by GCMS) as a colorless oil $(0.057 \mathrm{~g}, 55 \%)$ : ${ }^{1} \mathrm{H} \mathrm{NMR}\left(500 \mathrm{MHz}, \mathrm{CDCl}_{3}\right), \delta$ 7.28-7.24 (m, 2.66H), 7.17-7.14 (m, 3.71H), $5.81(\mathrm{ddt}, J=17.5,10.3,7.3,1.01 \mathrm{H}), 5.07-$ $5.01(\mathrm{~m}, 2.11 \mathrm{H}), 3.21(\mathrm{~s}, 0.13 \mathrm{H}), 3.16(\mathrm{~s}, 3 \mathrm{H}), 2.63-2.60(\mathrm{~m}, 2.49 \mathrm{H}), 2.33-2.30(\mathrm{~m}$, $0.08 \mathrm{H}), 2.18(\mathrm{dt}, J=7.4,1.2,2.11 \mathrm{H}), 1.84-1.81(\mathrm{~m}, 2.32 \mathrm{H}), 1.56-1.51(\mathrm{~m}, 4.61 \mathrm{H}), 1.28-$ $1.15(\mathrm{~m}, 5.77 \mathrm{H}) ;{ }^{13} \mathrm{C}$ NMR $\left(125 \mathrm{MHz}, \mathrm{CDCl}_{3}\right), \delta 143.1,134.0,128.3,128.2,125.5$, 117.2, 74.4, 48.1, 41.9, 38.9, 36.6, 33.4, 33.3, 27.8; IR (thin film) 2853, 1455, 1079, 699 $\mathrm{cm}^{-1}$; HRMS (EI) $m / z$ calcd for $\mathrm{C}_{17} \mathrm{H}_{23}\left(\mathrm{M}-\mathrm{CH}_{3} \mathrm{O}\right)^{+} 227.1800$, found 227.1809.

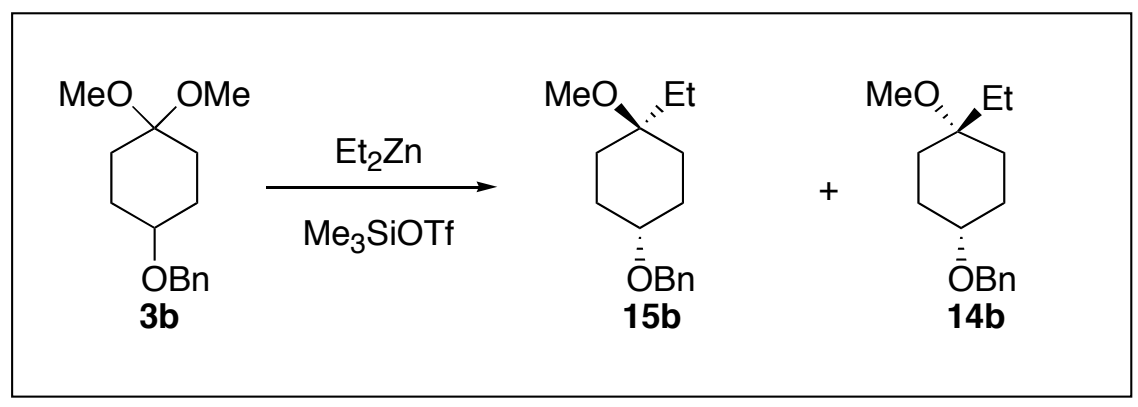

Benzyl Ethers $14 \mathbf{b} \&$ 15b: To a cooled $\left(-78{ }^{\circ} \mathrm{C}\right)$ solution of $\mathbf{3 b}(0.15 \mathrm{~g}, 0.60 \mathrm{mmol})$ in $\mathrm{CH}_{2} \mathrm{Cl}_{2}(3 \mathrm{~mL})$ was added a solution of $\mathrm{Et}_{2} \mathrm{Zn} 1 \mathrm{M}$ in hexanes $(0.7 \mathrm{~mL}, 0.7 \mathrm{mmol})$ and the mixture stirred for 10 seconds, then TMSOTf $(0.13 \mathrm{~mL}, 0.72 \mathrm{mmol})$ was added. The solution was allowed to warm $\left(0^{\circ} \mathrm{C}\right)$ and $\mathrm{NaHCO}_{3}(3 \mathrm{~mL}$ saturated, aqueous $)$ was added. The layers were separated and the aqueous layer was extracted with $\mathrm{CH}_{2} \mathrm{Cl}_{2}(5 \times 3 \mathrm{~mL})$. The organic layers were combined, filtered through $\mathrm{Na}_{2} \mathrm{SO}_{4}$, and concentrated in vacuo. A selectivity of $87: 13(\mathbf{1 4 b}: \mathbf{1 5 b})$ was determined by subjecting the unpurified oil to GC: $t_{R}$ (major) $15.2 \mathrm{~min}, t_{R}$ (minor) $14.6 \mathrm{~min}$. The resulting oil was purified by flash chromatography (97:3 hexanes/EtOAc) to afford the product (as a 8:1 mixture as determined by NMR) as a colorless oil $(0.11 \mathrm{~g}, 70 \%)$ : ${ }^{1} \mathrm{H}$ NMR $\left(400 \mathrm{MHz}, \mathrm{CDCl}_{3}\right) \delta$ 7.37-7.30 (m, 4.10H), 7.28-7.23 (m, 1.20H), $4.56(\mathrm{~s}, 2 \mathrm{H}), 4.50(\mathrm{~s}, 0.14 \mathrm{H}), 3.62-3.58(\mathrm{~m}$, 
$0.06 \mathrm{H}), 3.32(\mathrm{tt}, J=10.0,3.8,1 \mathrm{H}), 3.12(\mathrm{~s}, 0.18 \mathrm{H}), 3.11(\mathrm{~s}, 3 \mathrm{H}), 1.88-1.79(\mathrm{~m}, 3.96 \mathrm{H})$, $1.67-1.57(\mathrm{~m}, 2.40 \mathrm{H}), 1.49(\mathrm{q}, J=7.5,0.21 \mathrm{H}), 1.41(\mathrm{q}, J=7.5,2 \mathrm{H}), 1.18-1.10(\mathrm{~m}$, $1.96 \mathrm{H}), 0.85-0.81(\mathrm{~m}, 3.15 \mathrm{H}) ;{ }^{13} \mathrm{C}$ NMR $\left(125 \mathrm{MHz}, \mathrm{CDCl}_{3}\right) \delta$ 139.3, 139.2, 128.2, 127.4, 127.27, 127.25, 127.2, 77.2, 77.0, 74.7, 73.8, 73.7, 69.7, 48.2, 47.8, 31.3, 28.6, 28.5, 27.2, 25.9, 7.2, 6.9; IR (thin film) 2937, 1457, 1367, $1072 \mathrm{~cm}^{-1}$; HRMS (ES) $\mathrm{m} / \mathrm{z}$ calcd for $\mathrm{C}_{16} \mathrm{H}_{24} \mathrm{NaO}_{2}(\mathrm{M}+\mathrm{Na})^{+}$271.1674, found 271.1682. Anal. Calcd for $\mathrm{C}_{16} \mathrm{H}_{24} \mathrm{O}_{2}$ : C, 77.38; H, 9.74. Found: C, 77.57; H, 9.79.

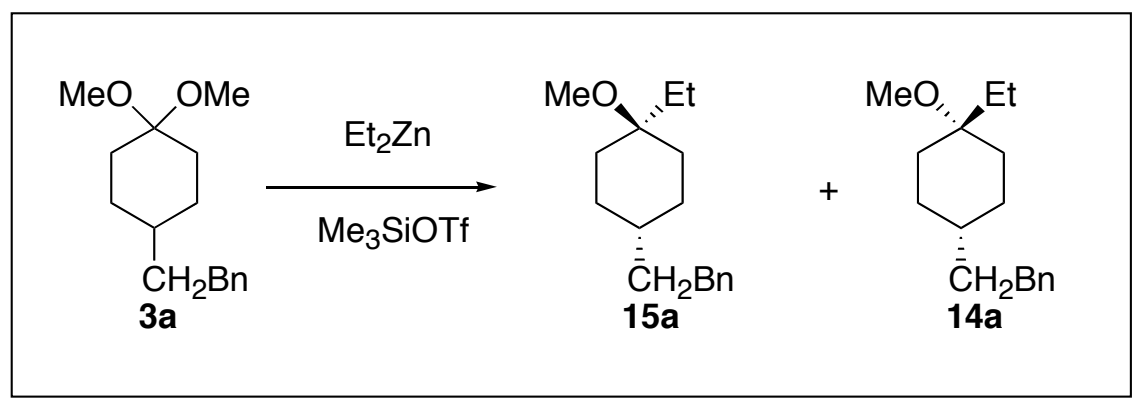

Methyl Ethers 14a \& 15a: To a cooled $\left(-78^{\circ} \mathrm{C}\right)$ solution of 3a $(0.15 \mathrm{~g}, 0.60 \mathrm{mmol})$ in $\mathrm{CH}_{2} \mathrm{Cl}_{2}(3 \mathrm{~mL})$ was added a solution of $\mathrm{Et}_{2} \mathrm{Zn}(1 \mathrm{M}$ in hexanes, $0.7 \mathrm{~mL}, 0.7 \mathrm{mmol})$ and the mixture was stirred for 10 seconds, then TMSOTf $(0.13 \mathrm{~mL}, 0.72 \mathrm{mmol})$ was added. The solution was allowed to warm $\left(0{ }^{\circ} \mathrm{C}\right)$ and $\mathrm{NaHCO}_{3}(3 \mathrm{~mL}$ saturated, aqueous) was added. The layers were separated and the aqueous layer was extracted with $\mathrm{CH}_{2} \mathrm{Cl}_{2}(5 \mathrm{x}$ $3 \mathrm{~mL}$ ). The organic layers were combined, filtered through $\mathrm{Na}_{2} \mathrm{SO}_{4}$, and concentrated in vacuo. A selectivity of 35:65 (15a : 14a) was determined by subjecting the unpurified oil to GCMS: $t_{R}$ (major) $16.3 \mathrm{~min}, \mathrm{t}_{\mathrm{R}}$ (minor) $17.0 \mathrm{~min}$. The resulting oil was purified by flash chromatography (97:3 hexanes/EtOAc) to afford the products (as a 2:1 mixture as determined by NMR) as a colorless oil $(0.12 \mathrm{~g}, 80 \%):{ }^{1} \mathrm{H}$ NMR $\left(500 \mathrm{MHz}, \mathrm{CDCl}_{3}\right) \delta$ 7.27-7.22 (m, 3.21H), 7.20-7.11 (m, 4.44H), $3.14(\mathrm{~s}, 3 \mathrm{H}), 3.09(\mathrm{~s}, 1.37 \mathrm{H}), 2.63-2.58$ $(2.96 \mathrm{H}), 1.83-1.79(\mathrm{~m}, 0.97 \mathrm{H}), 1.76-1.66(\mathrm{~m}, 4.19 \mathrm{H}), 1.56-1.52(\mathrm{~m}, 6.07 \mathrm{H}), 1.42-1.31$ $(4.12 \mathrm{H}), 1.29-1.18(\mathrm{~m}, 1.57 \mathrm{H}), 1.14-1.01(\mathrm{~m}, 3.01 \mathrm{H}), 0.84-0.80(\mathrm{~m}, 4.51 \mathrm{H}) ;{ }^{13} \mathrm{C} \mathrm{NMR}$ $\left(125 \mathrm{MHz}, \mathrm{CDCl}_{3}\right) \delta 143.1,142.9,128.29,128.25,128.24,128.18,125.6,125.5,75.8$, 74.4, 47.9, 47.8, 38.9, 37.6, 36.9, 36.1, 33.6, 33.3, 33.1, 32.8, 29.5, 28.9, 27.9, 24.3, 7.1, 6.7; IR (thin film) 2928, 1495, $1128 \mathrm{~cm}^{-1}$. Anal. Calcd for $\mathrm{C}_{17} \mathrm{H}_{26} \mathrm{O}: \mathrm{C}, 82.87 ; \mathrm{H}, 10.64$. Found: C, 82.96; H, 10.66 .

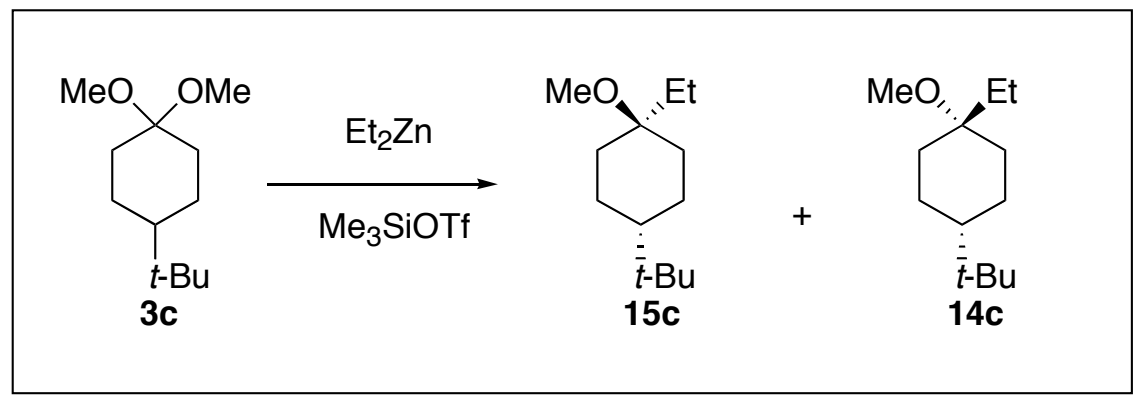

Methyl Ethers $14 \mathbf{c} \& 15 c:$ To a cooled $\left(-78{ }^{\circ} \mathrm{C}\right)$ solution of $3 \mathbf{c}(0.126 \mathrm{~g}, 0.600 \mathrm{mmol})$ in $\mathrm{CH}_{2} \mathrm{Cl}_{2}(3 \mathrm{~mL})$ was added diethylzinc $(1 \mathrm{M}$ in hexanes, $1.2 \mathrm{~mL}, 1.2 \mathrm{mmol})$ and the 
mixture was stirred for 10 seconds, then TMSOTf $(0.13 \mathrm{~mL}, 0.72 \mathrm{mmol})$ was added. The solution was allowed to warm $\left(0{ }^{\circ} \mathrm{C}\right)$ and $\mathrm{NaHCO}_{3}(3 \mathrm{~mL}$ saturated, aqueous) was added. The layers were separated and the aqueous layer was extracted with $\mathrm{CH}_{2} \mathrm{Cl}_{2}(5 \times 3 \mathrm{~mL})$. The organic layers were combined, filtered through $\mathrm{Na}_{2} \mathrm{SO}_{4}$, and concentrated in vacuo. A selectivity of $50: 50(\mathbf{1 4 c}: \mathbf{1 5 c})$ was determined by subjecting the unpurified oil to GC: $t_{R}$ (major) 8.5 min, $t_{R}$ (minor) 8.9 min. The resulting oil was purified by flash chromatography (98:2 hexanes/EtOAc) to afford the product (as a 50:50 mixture as determinded by NMR) as a colorless oil $(0.09 \mathrm{~g}, 75 \%)$ : ${ }^{1} \mathrm{H}$ NMR $\left(500 \mathrm{MHz}, \mathrm{CDCl}_{3}\right) \delta$ $3.16(\mathrm{~s}, 3 \mathrm{H}), 3.10(\mathrm{~s}, 3 \mathrm{H}), 1.89-1.79$, (m, $4 \mathrm{H}), 1.69-1.66(\mathrm{~m}, 2 \mathrm{H}), 1.56(\mathrm{q}, J=7.4,2 \mathrm{H})$, $1.51-1.49$ (m, 2H), 1.40 (q, $J=7.5 \mathrm{~Hz}, 2 \mathrm{H}), 1.35-1.20$ (m, 4H), 1.10 (ddd, $J=13.9,13.6$, 3.6, 2H), 1.05-1.00 (m, 3H), 0.96-0.90 (m, 1.5H), 0.85-0.80 (m, 26H); ${ }^{13} \mathrm{C}$ NMR $(125$ $\left.\mathrm{MHz}, \mathrm{CDCl}_{3}\right) \delta$ 75.9, 74.1, 48.05, 47.98, 47.82, 47.80, 34.3, 33.8, 32.4, 32.2, 29.6, 27.61, 27.59, 24.0, 23.0, 22.2, 7.1, 6.7; IR (thin film) 2933, 1456, $1367 \mathrm{~cm}^{-1}$; HRMS (ES) $\mathrm{m} / \mathrm{z}$ calcd for $\mathrm{C}_{13} \mathrm{H}_{26} \mathrm{O}(\mathrm{M})^{+}$198.1984, found 198.1981.

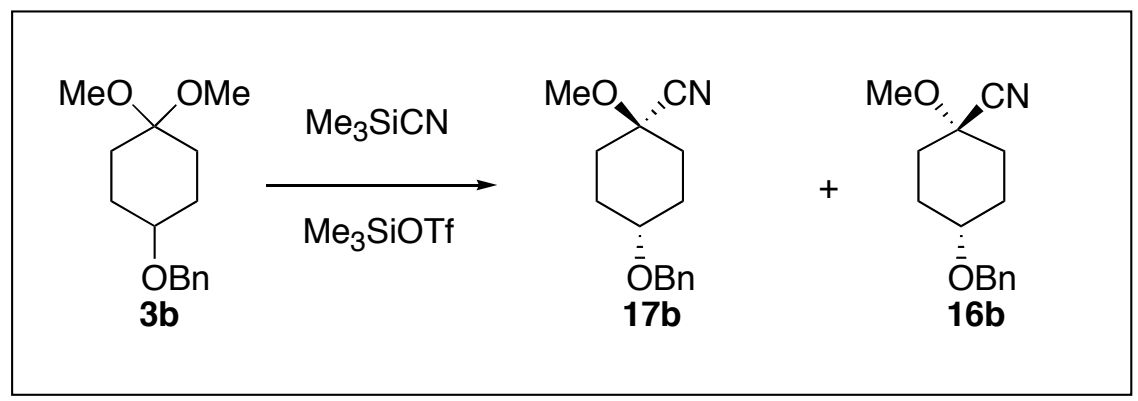

Cyanides 16b \& 17b: To a cooled $\left(-78^{\circ} \mathrm{C}\right)$ solution of $\mathbf{3 b}(0.150 \mathrm{~g}, 0.600 \mathrm{mmol})$ in $\mathrm{CH}_{2} \mathrm{Cl}_{2}(3 \mathrm{~mL})$ was added cyanotrimethylsilane $(0.31 \mathrm{~mL}, 2.4 \mathrm{mmol})$ and the mixture was stirred for 2 minutes, then TMSOTf $(0.13 \mathrm{~mL}, 0.72 \mathrm{mmol})$ was added. The solution was allowed to warm $\left(0{ }^{\circ} \mathrm{C}\right)$ and $\mathrm{NaHCO}_{3}(3 \mathrm{~mL}$ saturated, aqueous) was added. The layers were separated and the aqueous layer was extracted with $\mathrm{CH}_{2} \mathrm{Cl}_{2}(5 \times 3 \mathrm{~mL})$. The organic layers were combined, filtered through $\mathrm{Na}_{2} \mathrm{SO}_{4}$, and concentrated in vacuo. A selectivity of 24:76 (17b : 16b) was determined by subjecting the unpurified oil to GC: $t_{R}$ (major) $15.1 \mathrm{~min}, t_{R}$ (minor) $15.2 \mathrm{~min}$. The resulting oil was purified by flash chromatography ( $97: 3$ to $96: 4$ hexanes/EtOAc) to afford the products $\mathbf{1 7 b}$ and $\mathbf{1 7 b} \boldsymbol{\&} \mathbf{1 6 b}$ (as a 2:1 mixture as determined by NMR) separately as colorless oils. $\mathbf{1 7 b}(0.04 \mathrm{~g}, 24 \%)$ : ${ }^{1} \mathrm{H}$ NMR $\left(500 \mathrm{MHz}, \mathrm{CDCl}_{3}\right)$ 8 7.36-7.31 (m, 4H), 7.29-7.26 (m, 1H), $4.50(\mathrm{~s}, 2 \mathrm{H}), 3.60-$ $3.54(\mathrm{~m}, 1 \mathrm{H}), 3.44(\mathrm{~s}, 3 \mathrm{H}), 2.06-2.01(\mathrm{~m}, 2 \mathrm{H}), 1.94-1.88(\mathrm{~m}, 4 \mathrm{H}), 1.81-1.74(\mathrm{~m}, 2 \mathrm{H}) ;{ }^{13} \mathrm{C}$ NMR (125 MHz, $\left.\mathrm{CDCl}_{3}\right) \delta 138.6,128.4,127.5,127.3,119.6,74.7,72.2,69.9,52.5,30.5$, 25.6; IR (thin film) 2924, 2833, 1495, $1368 \mathrm{~cm}^{-1}$; HRMS (EI) $m / z$ calcd for $\mathrm{C}_{15} \mathrm{H}_{20} \mathrm{O}_{2} \mathrm{~N}$ $(\mathrm{M}+\mathrm{H})^{+}$246.1494, found 246.1483. Anal. Calcd for $\mathrm{C}_{15} \mathrm{H}_{19} \mathrm{O}_{2} \mathrm{~N}$ : C, 73.44; $\mathrm{H}, 7.81 ; \mathrm{N}$, 5.71. Found: C, 73.01; H, 7.92; N, 5.68. 17b \& 16b $(0.1 \mathrm{~g}, 65 \%):{ }^{1} \mathrm{H}$ NMR $(500 \mathrm{MHz}$, $\left.\mathrm{CDCl}_{3}\right), \delta 7.38-7.25(\mathrm{~m}, 7.39 \mathrm{H}), 4.52(\mathrm{~s}, 1.15 \mathrm{H}), 4.50(\mathrm{~s}, 1.74 \mathrm{H}), 3.60-3.54(\mathrm{~m}, 1 \mathrm{H})$, 3.54-3.48 (m, 0.48H), $3.44(\mathrm{~s}, 3.12 \mathrm{H}), 3.43(\mathrm{~s}, 1.17 \mathrm{H}), 2.25-2.17(\mathrm{~m}, 0.92 \mathrm{H}), 2.09-1.98$ $(\mathrm{m}, 2.10 \mathrm{H}), 1.98-1.85(\mathrm{~m}, 5.01 \mathrm{H}), 1.83-1.71(\mathrm{~m}, 3.9 \mathrm{H}) ;{ }^{13} \mathrm{C} \mathrm{NMR}\left(125 \mathrm{MHz}, \mathrm{CDCl}_{3}\right)$,

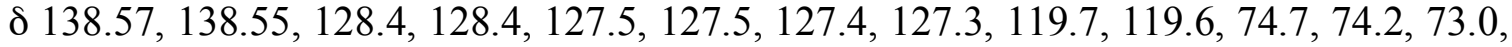
$72.2,70.1,69.9,52.8,52.4,30.5,26.6,26.0$; IR (thin film) 2941, 2866, 1495, $1028 \mathrm{~cm}^{-1}$; 
HRMS (EI) $m / z$ calcd for $\mathrm{C}_{15} \mathrm{H}_{20} \mathrm{O}_{2} \mathrm{~N}(\mathrm{M}+\mathrm{H})^{+}$246.1494, found 246.1503. Anal. Calcd for $\mathrm{C}_{15} \mathrm{H}_{19} \mathrm{O}_{2} \mathrm{~N}$ : C, 73.44; H, 7.81; N, 5.71. Found: C, 73.58; H, 7.88; N, 5.76.

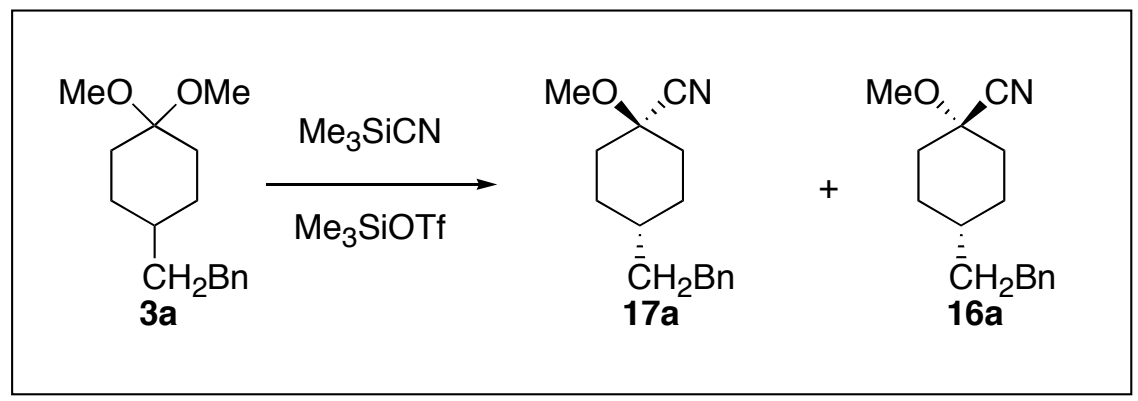

Cyanides 16a \& 17a: To a cooled $\left(-78{ }^{\circ} \mathrm{C}\right)$ solution of 3a $(0.150 \mathrm{~g}, 0.600 \mathrm{mmol})$ in $\mathrm{CH}_{2} \mathrm{Cl}_{2}(3 \mathrm{~mL})$ was added cyanotrimethylsilane $(0.09 \mathrm{~mL}, 0.7 \mathrm{mmol})$ and the mixture was stirred for 2 minutes, then TMSOTf $(0.13 \mathrm{~mL}, 0.72 \mathrm{mmol})$ was added. The solution was allowed to warm $\left(0{ }^{\circ} \mathrm{C}\right)$ and $\mathrm{NaHCO}_{3}(3 \mathrm{~mL}$ saturated, aqueous $)$ was added. The layers were separated and the aqueous layer was extracted with $\mathrm{CH}_{2} \mathrm{Cl}_{2}(5 \times 3 \mathrm{~mL})$. The organic layers were combined, filtered through $\mathrm{Na}_{2} \mathrm{SO}_{4}$, and concentrated in vacuo. A selectivity of $81: 19$ (17a : 16a) was determined by subjecting the unpurified oil to GC: $t_{R}$ (major) $15.1 \mathrm{~min}, \mathrm{t}_{\mathrm{R}}$ (minor) $15.2 \mathrm{~min}$. The resulting oil was purified by flash chromatography (83:17 to 75:25 hexanes/ $\mathrm{CH}_{2} \mathrm{Cl}_{2}$ ) to afford the product (as a 75:25 mixture as determined by NMR) as a colorless oil (0.14 g, 94\%): ${ }^{1} \mathrm{H} \mathrm{NMR} \mathrm{(500} \mathrm{MHz,}$ $\left.\mathrm{CDCl}_{3}\right) \delta 7.28-7.25(\mathrm{~m}, 2.50 \mathrm{H}), 7.18-7.14(\mathrm{~m}, 3.75 \mathrm{H}), 3.43(\mathrm{~s}, 3 \mathrm{H}), 3.38(\mathrm{~s}, 0.74 \mathrm{H}), 2.63-$ $2.58(\mathrm{~m}, 2.51 \mathrm{H}), 2.26-2.23(\mathrm{~m}, 2 \mathrm{H}), 2.18-2.15(\mathrm{~m}, 0.66 \mathrm{H}), 1.94-1.85(\mathrm{~m}, 2 \mathrm{H}), 1.70(\mathrm{td}, J$ $=13.6,3.9,0.51 \mathrm{H}), 1.64-1.51(\mathrm{~m}, 3.14 \mathrm{H}), 1.47-1.41(\mathrm{~m}, 2 \mathrm{H}), 1.35-1.43(\mathrm{~m}, 3.97 \mathrm{H}) ;{ }^{13} \mathrm{C}$ NMR $\left(125 \mathrm{MHz}, \mathrm{CDCl}_{3}\right) \delta 142.3,142.2,128.3,128.2$ 125.7, 125.6, 120.4, 119.4, 76.6, 73.0, 52.5, 52.3, 37.8, 37.7, 35.7, 35.0, 34.8, 33.2, 33.0, 33.0, 26.2; IR (thin film) 3022, 2928, 2859, 1495, $1106 \mathrm{~cm}^{-1}$; HRMS (EI) $\mathrm{m} / \mathrm{z}$ calcd for $\mathrm{C}_{15} \mathrm{H}_{21} \mathrm{O}(\mathrm{M}-\mathrm{CN})^{+} 217.1592$, found 217.1585. Anal. Calcd for $\mathrm{C}_{16} \mathrm{H}_{21} \mathrm{ON}$ : C, 78.97; H, 8.70; N, 5.76. Found: C, 79.05; H, 8.84; N, 5.84 .

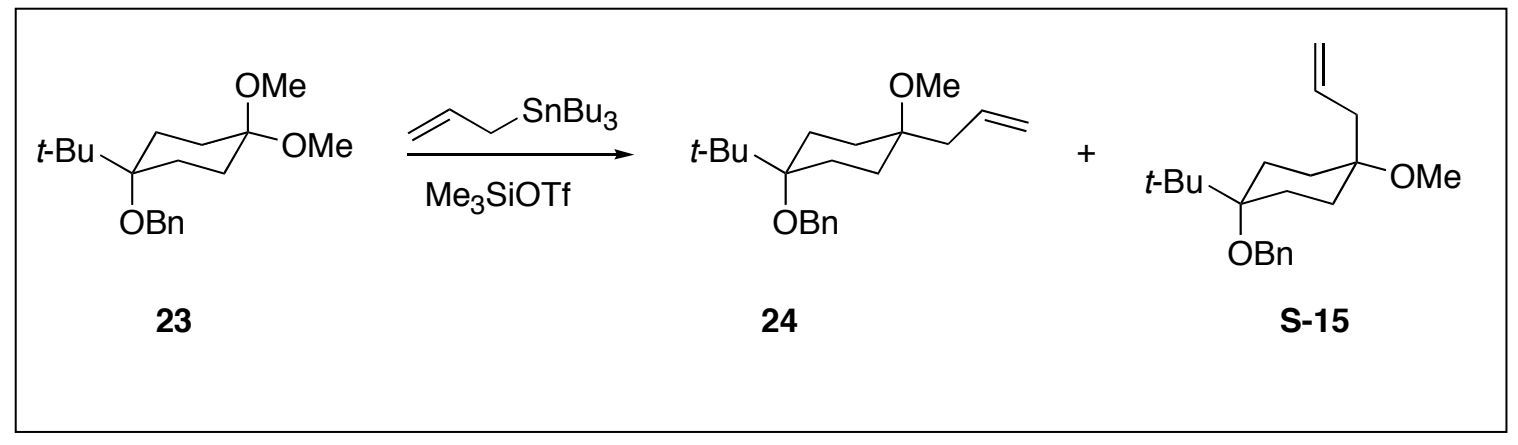

Benzyl Ethers 24 \& S-15: To a cooled $\left(-78^{\circ} \mathrm{C}\right)$ solution of $23(0.076 \mathrm{~g}, 0.248 \mathrm{mmol})$ in $\mathrm{CH}_{2} \mathrm{Cl}_{2}(2 \mathrm{~mL})$ was added allyltributyltin $(0.3 \mathrm{~mL}, 0.8 \mathrm{mmol})$ and the mixture was allowed to stir for 2 minutes, then TMSOTf $(0.05 \mathrm{~mL}, 0.4 \mathrm{mmol})$ was added. The solution was warmed $\left(0{ }^{\circ} \mathrm{C}\right)$ and $\mathrm{NaHCO}_{3}(2 \mathrm{~mL}$ saturated, aqueous $)$ was added. The layers were separated and the aqueous layer was extracted with $\mathrm{CH}_{2} \mathrm{Cl}_{2}(5 \times 2 \mathrm{~mL})$. The 
organic layers were combined, filtered through $\mathrm{Na}_{2} \mathrm{SO}_{4}$, and concentrated in vacuo. A selectivity of 82:18 (24: $\mathbf{S - 1 5})$ was determined by subjecting the unpurified oil to GC: $t_{R}$ (major) $18.1 \mathrm{~min}, t_{R}$ (minor) $17.7 \mathrm{~min}$. The resulting oil was purified by flash chromatography (99:1 to $98: 2$ hexanes/EtOAc) to afford the products $\mathbf{2 4}$ and $\mathbf{2 4} \boldsymbol{\&} \mathbf{S}-\mathbf{1 5}$ (as a 3.7:1 mixture as determined by NMR) separately as colorless oils. 24 (0.051 g, 65\%): ${ }^{1} \mathrm{H}$ NMR $\left(500 \mathrm{MHz}, \mathrm{CDCl}_{3}\right) \delta$ 7.36-7.30 (m, 4H), 7.25-7.22 (m, 1H), 5.74 (ddt, $J$ $=16.9,10.4,7.3,1 \mathrm{H}), 5.03-4.99(\mathrm{~m}, 2 \mathrm{H}), 4.61(\mathrm{~s}, 2 \mathrm{H}), 3.17(\mathrm{~s}, 3 \mathrm{H}), 2.16-2.14(\mathrm{~m}, 2 \mathrm{H})$, $1.79-1.60(\mathrm{~m}, 6 \mathrm{H}), 1.53(\mathrm{ddd}, J=13.8,13.6,4.6,2 \mathrm{H}), 1.02(\mathrm{~s}, 9 \mathrm{H}) ;{ }^{13} \mathrm{C}$ NMR $(125 \mathrm{MHz}$, $\left.\mathrm{CDCl}_{3}\right) \delta 140.4,133.9,128.2,126.8,126.7,117.2,79.7,73.9,65.7,48.4,41.7,38.7,29.3$, 27.1, 24.0; IR (thin film) 3071, 2957, 1639, 1496, 1368, $1082 \mathrm{~cm}^{-1}$. Anal. Calcd for $\mathrm{C}_{21} \mathrm{H}_{32} \mathrm{O}_{2}$ : C, 79.70; H, 10.19. Found: C, 79.54; H, 10.33. 24 \& S-15 (0.020 g, 15\%). ${ }^{1} \mathrm{H}$ NMR $\left(500 \mathrm{MHz}, \mathrm{CDCl}_{3}\right) \delta$ 7.40-7.31 (m, 5.25H), 7.27-7.22 (m, 3.53H), 5.87 (ddt, $J=$ $17.2,10.2,7.1,1 \mathrm{H}), 5.74(\mathrm{ddt}, J=17.6,10.4,7.3,0.27 \mathrm{H}), 5.12-5.06(\mathrm{~m}, 2 \mathrm{H}), 5.04-4.98$ $(\mathrm{m}, 0.57 \mathrm{H}), 4.65(\mathrm{~s}, 2 \mathrm{H}), 4.62(\mathrm{~s}, 0.54 \mathrm{H}), 3.18-3.17(\mathrm{~m}, 3.91 \mathrm{H}), 2.34-2.33(\mathrm{~m}, 2 \mathrm{H}), 2.16-$ $2.15(\mathrm{~m}, 0.55 \mathrm{H}), 1.99-1.94(\mathrm{~m}, 2 \mathrm{H}), 1.80-1.66(\mathrm{~m}, 3.5 \mathrm{H}), 1.66-1.48(\mathrm{~m}, 6.29 \mathrm{H}) 1.03-1.02$ $(\mathrm{m}, 11.88 \mathrm{H}) ;{ }^{13} \mathrm{C} \mathrm{NMR}\left(125 \mathrm{MHz}, \mathrm{CDCl}_{3}\right) \delta 140.2,140.1,134.2,133.9,128.3,128.2$, $126.94,126.91,126.86,126.7,117.2,117.0,79.7,79.1,76.8,75.4,65.7,65.7,48.4,48.3$, 41.8, 41.7, 38.7, 36.1, 29.7, 29.4, 27.1, 26.0, 24.0; IR (thin film) 3070, 3029, 2957, 1638, $1496,1454 \mathrm{~cm}^{-1}$. Anal. Calcd for $\mathrm{C}_{21} \mathrm{H}_{32} \mathrm{O}_{2}$ : C, 79.70; H, 10.19. Found: C, 79.47; $\mathrm{H}$, 10.40 .
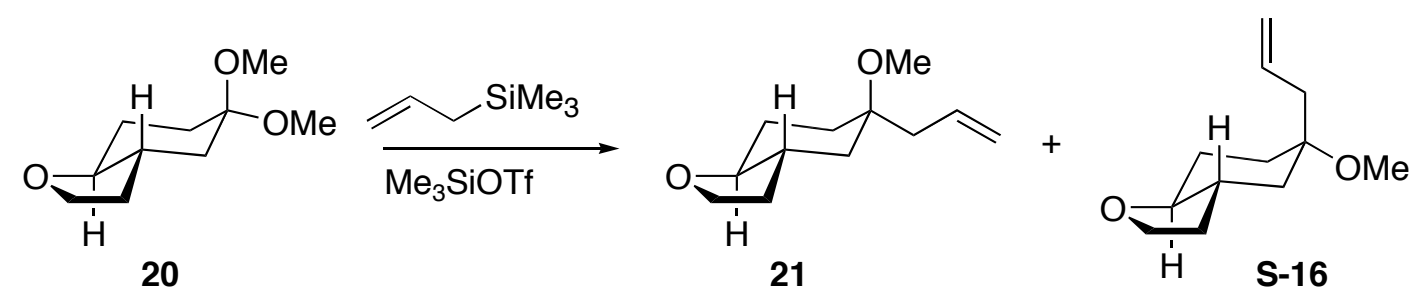

Methyl Ethers 21 \& S-16: To a cooled $\left(-78{ }^{\circ} \mathrm{C}\right)$ solution of $20(0.10 \mathrm{~g}, 0.50 \mathrm{mmol})$ in $\mathrm{CH}_{2} \mathrm{Cl}_{2}(2 \mathrm{~mL})$ was added allyltrimethylsilane $(0.35 \mathrm{~mL}, 2.1 \mathrm{mmol})$ and the mixture was allowed to stir for 2 minutes, then TMSOTf $(0.12 \mathrm{~mL}, 0.64 \mathrm{mmol})$ was added. The solution was allowed to warm $\left(0{ }^{\circ} \mathrm{C}\right)$, and $\mathrm{NaHCO}_{3}(2 \mathrm{~mL}$ saturated, aqueous) was added. The layers were separated and the aqueous layer was extracted with $\mathrm{CH}_{2} \mathrm{Cl}_{2}(5 \times 2 \mathrm{~mL})$. The organic layers were combined, filtered through $\mathrm{Na}_{2} \mathrm{SO}_{4}$, and concentrated in vacuo. A selectivity of 90:10 (21 : S-16) was determined by NMR. The resulting oil was purified by flash chromatography ( $99: 1$ hexanes/EtOAc) to afford the products $\mathbf{2 1}$ and $\mathbf{2 1}$ \& S-16 (as a 5:1 mixture as determined by NMR) separately as colorless oils. 21 (0.03 g, 26\%): ${ }^{1} \mathrm{H}$ NMR $\left(500 \mathrm{MHz}, \mathrm{CDCl}_{3}\right) \delta 5.81$ (ddt, $\left.J=17.4,10.3,7.3,1 \mathrm{H}\right), 5.10-5.04(\mathrm{~m}$, $2 \mathrm{H}), 3.94-3.89(\mathrm{~m}, 2 \mathrm{H}), 3.19(\mathrm{~s}, 3 \mathrm{H}), 2.99(\mathrm{ddd}, J=11.2,10.2,3.9,1 \mathrm{H}), 2.28$ (ddt, $J=$ $14.4,7.2,1.3,1 \mathrm{H}), 2.20(\mathrm{ddt}, J=14.4,7.2,1.3,1 \mathrm{H}), 2.10(\mathrm{dt}, J=13.6,3.1,1 \mathrm{H}), 1.99$ $1.88(\mathrm{~m}, 3 \mathrm{H}), 1.83-1.74(\mathrm{~m}, 1 \mathrm{H}), 1.63-1.48(\mathrm{~m}, 2 \mathrm{H}), 1.24$ (ddd, $J=14.4,13.3,4.0,1 \mathrm{H})$, $1.08(\mathrm{dd}, J=13.5,12.6,1 \mathrm{H}) ;{ }^{13} \mathrm{C}$ NMR $\left(125 \mathrm{MHz}, \mathrm{CDCl}_{3}\right) \delta 133.6,117.6,83.1,75.5$, 67.3, 48.1 4 41.3, 39.8, 36.5, 31.7, 30.5, 26.4; IR (thin film) 3074, 2936, 1639, 1456, 1358, $1076 \mathrm{~cm}^{-1}$; HRMS (EI) $m / z$ calcd for $\mathrm{C}_{12} \mathrm{H}_{21} \mathrm{O}_{2}(\mathrm{M}+\mathrm{H})^{+} 197.1542$, found 197.1549 . Anal. Calcd for $\mathrm{C}_{12} \mathrm{H}_{20} \mathrm{O}_{2}$ : C, 73.43; H, 10.27. Found: C, 73.72; H, 10.28. 21 \& S-16 
(0.06 g, $54 \%):{ }^{1} \mathrm{H}$ NMR (500 MHz, $\left.\mathrm{CDCl}_{3}\right) \delta 5.87-5.76(\mathrm{~m}, 1.18 \mathrm{H}), 5.14-5.04(\mathrm{~m}$, $2.43 \mathrm{H}), 3.98-3.81(\mathrm{~m}, 2.44 \mathrm{H}), 3.24(\mathrm{~s}, 0.54 \mathrm{H}), 3.19(\mathrm{~s}, 3 \mathrm{H}), 3.09$ (ddd, $J=10.7,9.9,3.9$, $0.20 \mathrm{H}), 2.99(\mathrm{ddd}, J=11.1,10.3,3.9,1 \mathrm{H}), 2.43-2.42(\mathrm{~m}, 0.34 \mathrm{H}), 2.28(\mathrm{ddt}, J=14.6,7.2$, $1.2,1 \mathrm{H}), 2.20(\mathrm{ddt}, J=14.2,7.3,1.2,1 \mathrm{H}), 2.12-2.00(\mathrm{~m}, 1.49 \mathrm{H}), 1.99-1.88(\mathrm{~m}, 3.67 \mathrm{H})$, 1.79 (tddd, $J=12.4,10.0,6.6,3.3,1 \mathrm{H}), 1.63-1.36(\mathrm{~m}, 2.96 \mathrm{H}), 1.32-1.21(\mathrm{~m}, 1.63 \mathrm{H}), 1.08$ $(\mathrm{t}, J=13.4,12.6,1 \mathrm{H}) ;{ }^{13} \mathrm{C}$ NMR $\left(125 \mathrm{MHz}, \mathrm{CDCl}_{3}\right) \delta 133.6,133.4,117.6,117.5,83.1$, 83.1, 75.5, 67.7, 67.2, 48.8, 48.1, 41.6, 41.3, 39.7, 37.1, 36.4, 36.4, 32.4, 31.7, 30.7, 30.4, 27.0, 26.4; IR (thin film) 3074, 2938, 1640, 1358, $1075 \mathrm{~cm}^{-1}$; HRMS (EI) $\mathrm{m} / \mathrm{z}$ calcd for $\mathrm{C}_{12} \mathrm{H}_{21} \mathrm{O}_{2}(\mathrm{M}+\mathrm{H})^{+}$197.1542, found 197.1549. Anal. Calcd for $\mathrm{C}_{12} \mathrm{H}_{20} \mathrm{O}_{2}: \mathrm{C}, 73.43 ; \mathrm{H}$, 10.27. Found: C, 73.68; $\mathrm{H}, 10.55$.

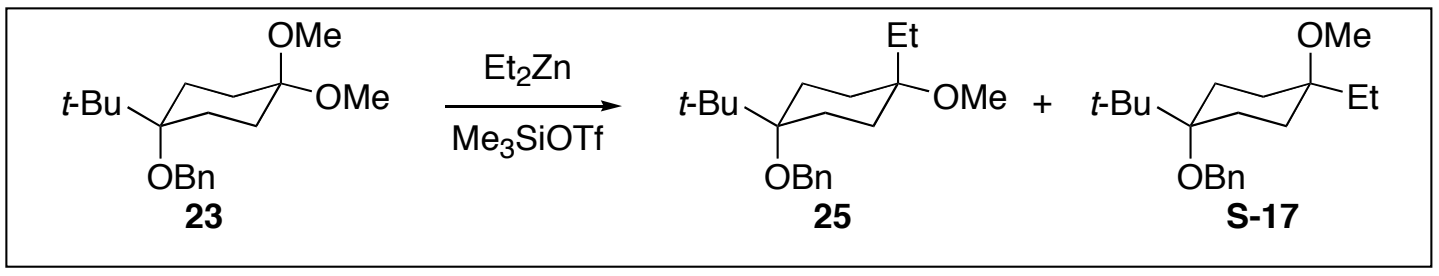

Benzyl Ethers 25 \& S-17: To a cooled $\left(-78^{\circ} \mathrm{C}\right)$ solution of $23(0.100 \mathrm{~g}, 0.33 \mathrm{mmol})$ in $\mathrm{CH}_{2} \mathrm{Cl}_{2}(2 \mathrm{~mL})$ was added a solution of $\mathrm{Et}_{2} \mathrm{Zn}(1 \mathrm{M}$ in hexanes, $0.39 \mathrm{~mL}, 0.39 \mathrm{mmol})$ and the mixture was allowed to stir for 10 seconds, then TMSOTf $(0.09 \mathrm{~mL}, 0.39 \mathrm{mmol})$ was added. The solution was allowed to warm $\left(0{ }^{\circ} \mathrm{C}\right)$ and $\mathrm{NaHCO}_{3}(2 \mathrm{~mL}$, saturated, aqueous) was added. The layers were separated and the aqueous layer was extracted with $\mathrm{CH}_{2} \mathrm{Cl}_{2}(5 \times 2 \mathrm{~mL})$. The organic layers were combined, filtered through $\mathrm{Na}_{2} \mathrm{SO}_{4}$, and concentrated in vacuo. A selectivity of 97:3 (25: S-17) was determined by subjecting the unpurified oil to GCMS: $t_{R}$ (major) 19.4 min, $t_{R}$ (minor) 19.0 min. The resulting oil was purified by flash chromatography (98:2 to $97: 3$ hexanes/EtOAc) to afford the product as a colorless oil $(0.078 \mathrm{~g}, 79 \%)$ : ${ }^{1} \mathrm{H}$ NMR $\left(500 \mathrm{MHz}, \mathrm{CDCl}_{3}\right) \delta$ 7.41-7.39 (m, 2H), 7.34-7.31 $(\mathrm{m}, 2 \mathrm{H}), 7.26-7.22(\mathrm{~m}, 1 \mathrm{H}), 4.66(\mathrm{~s}, 2 \mathrm{H}), 3.13(\mathrm{~s}, 3 \mathrm{H}), 1.98-1.92(\mathrm{~m}, 2 \mathrm{H}), 1.72$ (ddd, $J=$ $13.6,13.5,3.9,2 \mathrm{H}), 1.62-1.55(\mathrm{~m}, 4 \mathrm{H}), 1.48(\mathrm{td}, J=14.3,4.0,2 \mathrm{H}), 1.01(\mathrm{~s}, 9 \mathrm{H}), 0.85(\mathrm{t}$, $J=7.4,3 \mathrm{H}) ;{ }^{13} \mathrm{C}$ NMR $\left(125 \mathrm{MHz}, \mathrm{CDCl}_{3}\right) \delta 140.1,128.2,126.9,79.3,75.4,65.6,48.0$, 38.7, 29.6, 27.2, 26.1, 23.2, 6.9; IR (thin film) 2963, 2875, 1120, $1070 \mathrm{~cm}^{-1}$. Anal. Calcd for $\mathrm{C}_{20} \mathrm{H}_{32} \mathrm{O}_{2}$ : C, 78.90; H, 10.59. Found: C, 79.00; H, 10.56 .

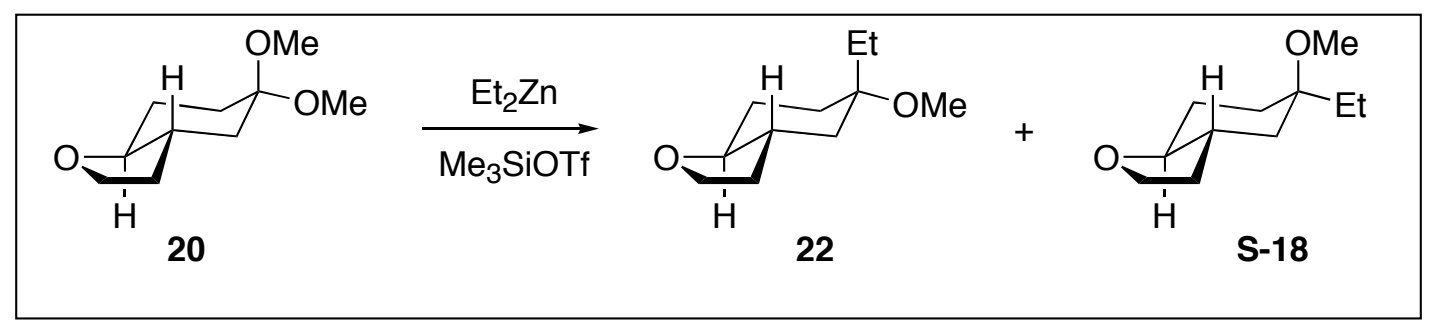

Methyl Ethers S-18 \& 22: To a cooled $\left(-78{ }^{\circ} \mathrm{C}\right)$ solution of $20(0.10 \mathrm{~g}, 0.50 \mathrm{mmol})$ in $\mathrm{CH}_{2} \mathrm{Cl}_{2}(2 \mathrm{~mL})$ was added a solution of $\mathrm{Et}_{2} \mathrm{Zn}$ ( $1 \mathrm{M}$ in hexanes, $\left.0.64 \mathrm{~mL}, 0.64 \mathrm{mmol}\right)$ and the mixture was allowed to stir for 10 seconds, then TMSOTf $(0.12 \mathrm{~mL}, 0.64 \mathrm{mmol})$ was added. The solution was allowed to warm $\left(0{ }^{\circ} \mathrm{C}\right)$, and $\mathrm{NaHCO}_{3}(2 \mathrm{~mL}$ saturated, aqueous) was added. The layers were separated and the aqueous layer was extracted with 
$\mathrm{CH}_{2} \mathrm{Cl}_{2}(5 \times 2 \mathrm{~mL})$. The organic layers were combined, filtered through $\mathrm{Na}_{2} \mathrm{SO}_{4}$, and concentrated in vacuo. A selectivity of 88:12 (22: S-18) was determined by subjecting the unpurified oil to $\mathrm{GC}: \mathrm{t}_{\mathrm{R}}$ (major) $9.3 \mathrm{~min}, \mathrm{t}_{\mathrm{R}}$ (minor) $9.1 \mathrm{~min}$. The resulting oil was purified by flash chromatography ( $99: 1$ hexanes/EtOAc) to afford the products 22 and 22 \& S-18 (as a 5:1 mixture as determined by NMR) separately as colorless oils. $52(0.05 \mathrm{~g}$, 45\%): ${ }^{1} \mathrm{H}$ NMR $\left(500 \mathrm{MHz}, \mathrm{CDCl}_{3}\right), \delta 3.98-3.91(\mathrm{~m}, 2 \mathrm{H}), 3.18(\mathrm{~s}, 3 \mathrm{H}), 3.09$ (ddd, $J=$ $10.5,10.0,3.9,1 \mathrm{H}), 2.06(\mathrm{dt}, J=12.4,2.8,1 \mathrm{H}), 2.04-1.93(\mathrm{~m}, 2 \mathrm{H}), 1.89(\mathrm{dtd}, J=12.2$, $2.8,2.5,1 \mathrm{H}), 1.66(\mathrm{q}, J=7.4,2 \mathrm{H}), 1.61-1.34(\mathrm{~m}, 4 \mathrm{H}), 1.25(\mathrm{t}, J=12.5,1 \mathrm{H}), 0.85(\mathrm{t}, J=$ 7.4, 3H); ${ }^{13} \mathrm{C}$ NMR $\left(125 \mathrm{MHz}, \mathrm{CDCl}_{3}\right) \delta 83.4,77.2,67.7,48.5,41.7,36.2,32.3,30.8$, 27.2, 24.6, 6.8; IR (thin film) 2937, 1460, 1357, $1257 \mathrm{~cm}^{-1}$; HRMS (EI) $\mathrm{m} / z$ calcd for $\mathrm{C}_{9} \mathrm{H}_{15} \mathrm{O}_{2}\left(\mathrm{M}-\mathrm{C}_{2} \mathrm{H}_{5}\right)^{+}$155.1072, found 155.1078. 22 \& S-18 $(0.03 \mathrm{~g}, 33 \%){ }^{1} \mathrm{H}$ NMR $(500$ $\left.\mathrm{MHz}, \mathrm{CDCl}_{3}\right) \delta$ 3.98-3.91 (m, 2H), $3.18(\mathrm{~s}, 3 \mathrm{H}), 3.13(\mathrm{~s}, 0.58 \mathrm{H}), 3.09$ (ddd, $J=10.4$, $10.3,3.9,1 \mathrm{H}), 3.04-3.98(\mathrm{~m}, 0.23 \mathrm{H}), 2.13-1.86(\mathrm{~m}, 4.83 \mathrm{H}), 1.84-1.74(\mathrm{~m}, 0.44 \mathrm{H}), 1.66$ $(\mathrm{q}, J=7.4,2 \mathrm{H}), 1.61-1.34(\mathrm{~m}, 5.01 \mathrm{H}), 1.25(\mathrm{t}, J=12.5,1 \mathrm{H}), 1.21-1.14(\mathrm{~m}, 0.41 \mathrm{H}), 1.03-$ $0.98(\mathrm{~m}, 0.23 \mathrm{H}), 0.87-0.84(\mathrm{~m}, 3.54 \mathrm{H}) ;{ }^{13} \mathrm{C} \mathrm{NMR}\left(125 \mathrm{MHz}, \mathrm{CDCl}_{3}\right), \delta 83.38,83.36$, 77.2, 75.7, 67.7, 67.2, 48.5, 47.9, 41.7, 39.8, 36.2, 36.1, 32.3, 31.6, 30.8, 30.5, 29.1, 27.2, 26.5, 7.3, 6.8; IR (thin film) 2937, 1461, 1357, $1128 \mathrm{~cm}^{-1}$; HRMS (EI) $\mathrm{m} / \mathrm{z}$ calcd for $\mathrm{C}_{10} \mathrm{H}_{17} \mathrm{O}(\mathrm{M}-\mathrm{MeO})^{+}$153.1279, found 153.1279. Anal. Calcd for $\mathrm{C}_{11} \mathrm{H}_{20} \mathrm{O}_{2}: \mathrm{C}, 71.70 ; \mathrm{H}$, 10.94. Found: C, $71.75 ; \mathrm{H}, 10.73$.

\section{Proofs of Stereochemistry}

1. Proof of Stereochemistry for $\mathbf{1 2 b}$.

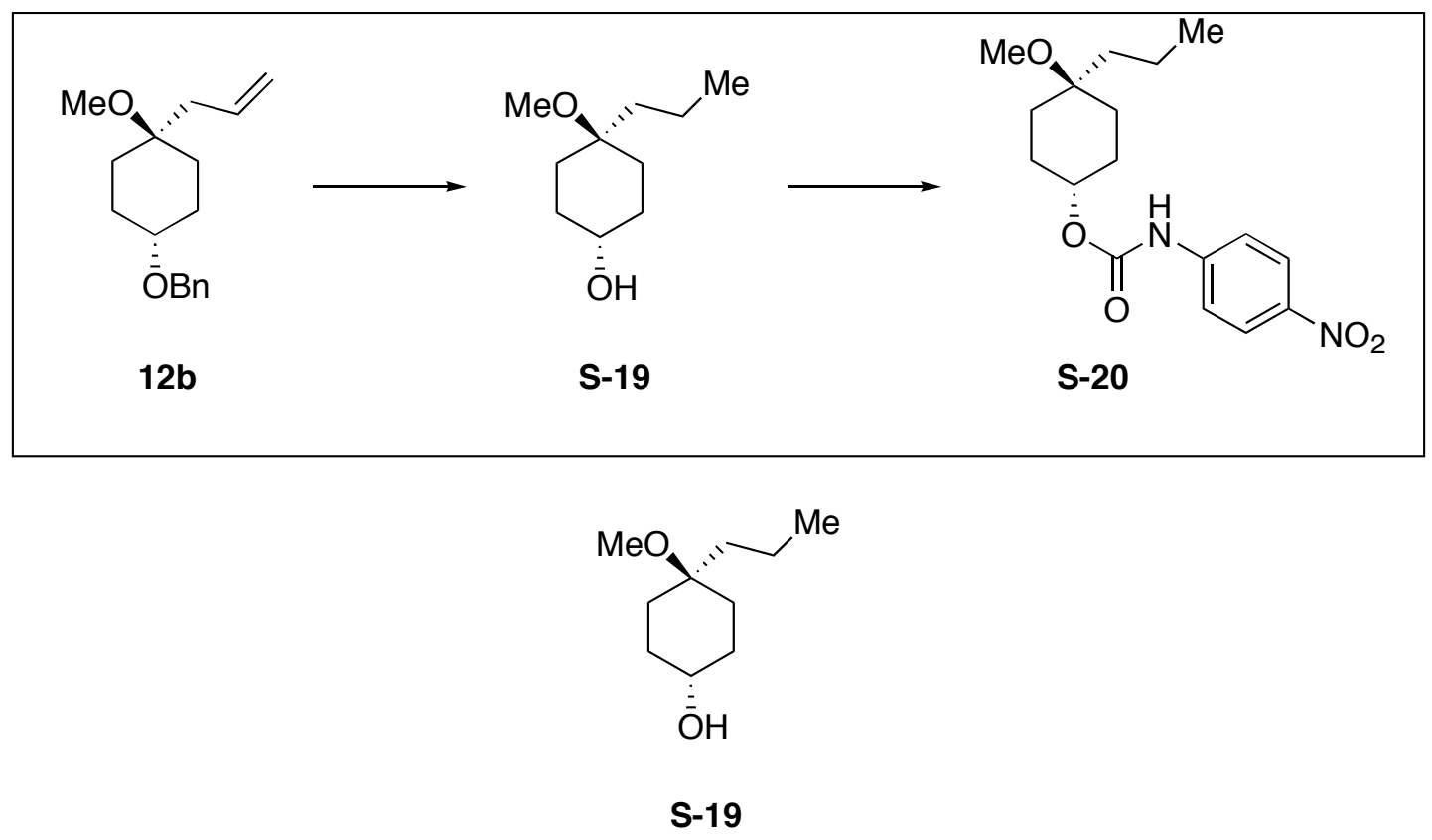

Alcohol S-19: To a solution of $12 \mathbf{b}(0.053 \mathrm{~g}, 0.20 \mathrm{mmol})$ in ethanol $(1 \mathrm{~mL})$ was added $\mathrm{Pd} / \mathrm{C}(10 \%, 0.005 \mathrm{~g}, 0.002 \mathrm{mmol})$. The mixture was first purged with $\mathrm{H}_{2}$ then placed under $\mathrm{H}_{2}(1 \mathrm{~atm})$ and stirred for 4 days. The mixture was filtered and the solvent was removed in vacuo. The resulting oil was purified by flash chromatography $(83: 17$ hexanes/EtOAc) to afford the product as colorless oil $(0.027 \mathrm{~g}, 77 \%)$ : ${ }^{1} \mathrm{H}$ NMR (500 
$\left.\mathrm{MHz}, \mathrm{CDCl}_{3}\right) \delta 3.94(\mathrm{~m}, 1 \mathrm{H}), 3.12(\mathrm{~s}, 3 \mathrm{H}), 1.81(\mathrm{ddt}, J=14.1,10.8,3.5,2 \mathrm{H}), 1.65(\mathrm{~m}$, $2 \mathrm{H}), 1.56-1.43(\mathrm{~m}, 7 \mathrm{H}), 1.35-1.25(\mathrm{~m}, 2 \mathrm{H}), 0.92(\mathrm{t}, J=7.3,3 \mathrm{H}) ;{ }^{13} \mathrm{C}$ NMR $(125 \mathrm{MHz}$, $\left.\mathrm{CDCl}_{3}\right) \delta 74.8,48.3,37.8,34.3,29.2,28.7,15.7,14.6$; IR (thin film) 3389, 2942, 1498, $1073 \mathrm{~cm}^{-1}$; HRMS (CI) $\mathrm{m} / z$ calcd for $\mathrm{C}_{10} \mathrm{H}_{24} \mathrm{NO}_{2}\left(\mathrm{M}+\mathrm{NH}_{4}\right)^{+} 190.1807$, found 190.1803. Anal. Calcd for $\mathrm{C}_{10} \mathrm{H}_{20} \mathrm{O}_{2}$ : C, 69.72; H, 11.70. Found: C, 69.60; H, 11.90.

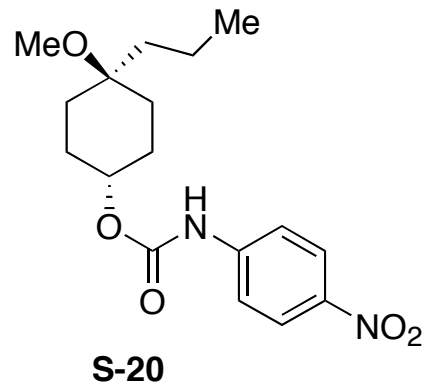

Carbamate S-20: To a solution of S-19 $(0.046 \mathrm{~g}, 0.27 \mathrm{mmol})$ in THF $(1 \mathrm{~mL})$ was added $p$-phenylnitroisocyanate $(0.049 \mathrm{~g}, 0.30 \mathrm{mmol})$ and the mixture was stirred for 2 days. The solvent was removed in vacuo, and the resulting solid was dissolved in $\mathrm{CH}_{2} \mathrm{Cl}_{2}(0.5$ $\mathrm{mL}$ ) and subjected to flash chromatography (91:9 to 83:17 hexanes/EtOAc) to afford the product as a colorless solid $(0.073 \mathrm{~g}, 80 \%)$ : $\mathrm{mp} 144{ }^{\circ} \mathrm{C} ;{ }^{1} \mathrm{H}$ NMR $\left(500 \mathrm{MHz}, \mathrm{CDCl}_{3}\right) \delta$ $8.20(\mathrm{~d}, J=9.2,2 \mathrm{H}), 7.57(\mathrm{~d}, J=9.2,2 \mathrm{H}), 7.11(\mathrm{br} \mathrm{s}, 1 \mathrm{H}), 5.04(\mathrm{br} \mathrm{s}, 1 \mathrm{H}), 3.14(\mathrm{~s}, 3 \mathrm{H})$, 1.87 (tt, $J=11.4,2.9,2 \mathrm{H}), 1.74-1.66(\mathrm{~m}, 4 \mathrm{H}), 1.53(\mathrm{ddd}, J=13.7,13.3,3.7,2 \mathrm{H}), 1.45-$ $1.42(\mathrm{~m}, 2 \mathrm{H}), 1.38-1.26(\mathrm{~m}, 2 \mathrm{H}), 0.93(\mathrm{t}, J=7.2,3 \mathrm{H}) ;{ }^{13} \mathrm{C} \mathrm{NMR}\left(125 \mathrm{MHz}, \mathrm{CDCl}_{3}\right) \delta$ $152.5,144.2,142.8,125.2,117.6,74.1,72.2,48.3,38.7,28.6,25.7,15.7,14.6$; IR (KBr) $3367,2963,2910,1732,1609,1540,1328 \mathrm{~cm}^{-1}$; HRMS (EI) $\mathrm{m} / z$ calcd for $\mathrm{C}_{17} \mathrm{H}_{24} \mathrm{~N}_{2} \mathrm{O}_{5}$ (M) 336.1685, found 336.1684. Anal. Calcd for $\mathrm{C}_{17} \mathrm{H}_{24} \mathrm{~N}_{2} \mathrm{O}_{5}$ : C, 60.70; H, 7.19; N, 8.33. Found: C, 61.09; H, 7.18; N, 8.36.

\section{Proof of Stereochemistry for $6 b$.}

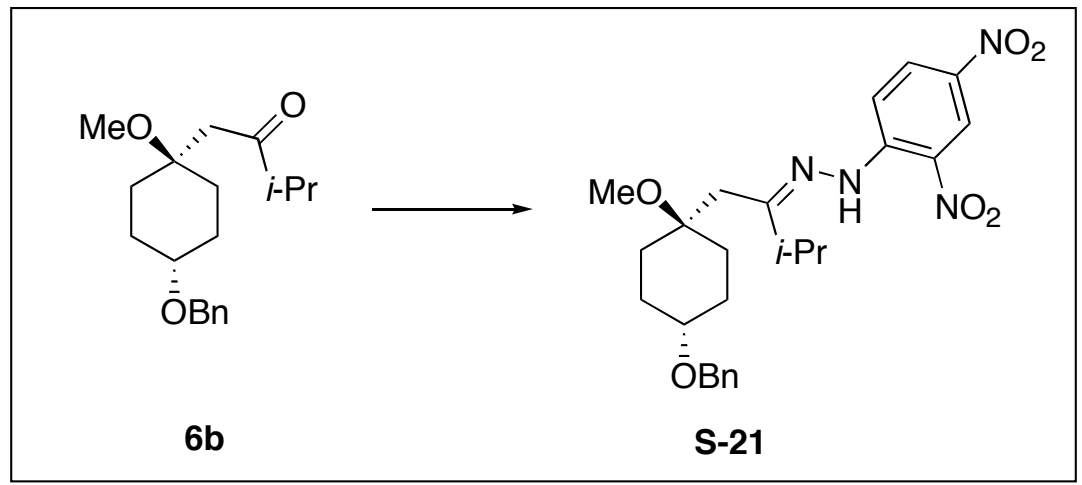

Hydrazone S-21: To a solution of $6 \mathbf{b}(0.085 \mathrm{~g}, 0.34 \mathrm{mmol})$ in ethanol $(4 \mathrm{~mL})$ was added 1 drop of $\mathrm{H}_{2} \mathrm{SO}_{4}$ (concentrated) and 2,4-dinitrophenylhyrdazine $(0.14 \mathrm{~g}, 0.68 \mathrm{mmol})$. The mixture was allowed to stir for 3 days, then $\mathrm{NaHCO}_{3}$ ( $1 \mathrm{~mL}$ saturated, aqueous) was added along with $\mathrm{CH}_{2} \mathrm{Cl}_{2}(5 \mathrm{~mL})$. The layers were separated and the aqueous layer was 
washed with $\mathrm{CH}_{2} \mathrm{Cl}_{2}(5 \times 5 \mathrm{~mL})$. The organic layers were combined, filtered through $\mathrm{Na}_{2} \mathrm{SO}_{4}$, and concentrated in vacuo. The resulting orange oil was purified by flash chromatography (50:50 to $0: 100$ hexanes/ $\mathrm{CH}_{2} \mathrm{Cl}_{2}$ ) to afford the product as an orange solid (0.11 g, 67\%): mp $127^{\circ} \mathrm{C} ;{ }^{1} \mathrm{H}$ NMR $\left(500 \mathrm{MHz}, \mathrm{CDCl}_{3}\right) \delta 11.87$ (br s, $\left.1 \mathrm{H}\right), 9.12(\mathrm{~d}, J=$ 2.6, 1H), 8.26 (ddd, $J=9.6,2.6,0.6,1 \mathrm{H}), 7.92(\mathrm{~d}, J=9.6,1 \mathrm{H}), 7.37-7.26(\mathrm{~m}, 5 \mathrm{H}), 4.54$ (s, 2H), 3.51 (ddd, $J=11.2,7.4,3.5,1 \mathrm{H}), 3.27$ (s, 3H), 2.69 (s, 2H), 2.61 (dt, $J=13.4$, 6.6, $1 \mathrm{H}), 2.05-1.95(\mathrm{~m}, 2 \mathrm{H}), 1.85(\mathrm{ddd}, J=14.3,10.2,3.9,2 \mathrm{H}), 1.70-1.62(\mathrm{~m}, 4 \mathrm{H}), 1.24$ $(\mathrm{d}, J=6.8,6 \mathrm{H}) ;{ }^{13} \mathrm{C} \mathrm{NMR}\left(125 \mathrm{MHz}, \mathrm{CDCl}_{3}\right), \delta 164.2,145.8,138.6,137.2,129.5,128.4$, 127.6, 127.5, 123.6, 116.6, 78.3, 74.5, 70.2, 48.5, 37.7, 37.4, 29.5, 27.9, 20.8; IR (KBr) 3200, 2933, 2845, 1611, 1583, 1517, 1417, $1328 \mathrm{~cm}^{-1}$; HRMS (ES) $\mathrm{m} / \mathrm{z}$ calcd for $\mathrm{C}_{25} \mathrm{H}_{32} \mathrm{~N}_{4} \mathrm{NaO}_{6}(\mathrm{M}+\mathrm{Na})^{+}$507.2220, found 507.2233. Anal. Calcd for $\mathrm{C}_{25} \mathrm{H}_{32} \mathrm{~N}_{4} \mathrm{O}_{6}: \mathrm{C}$, 61.97; H, 6.66; N, 11.56. Found: C, 62.00; H, 6.76; N, 11.57 .

3. Proof of Stereochemistry for 11 .

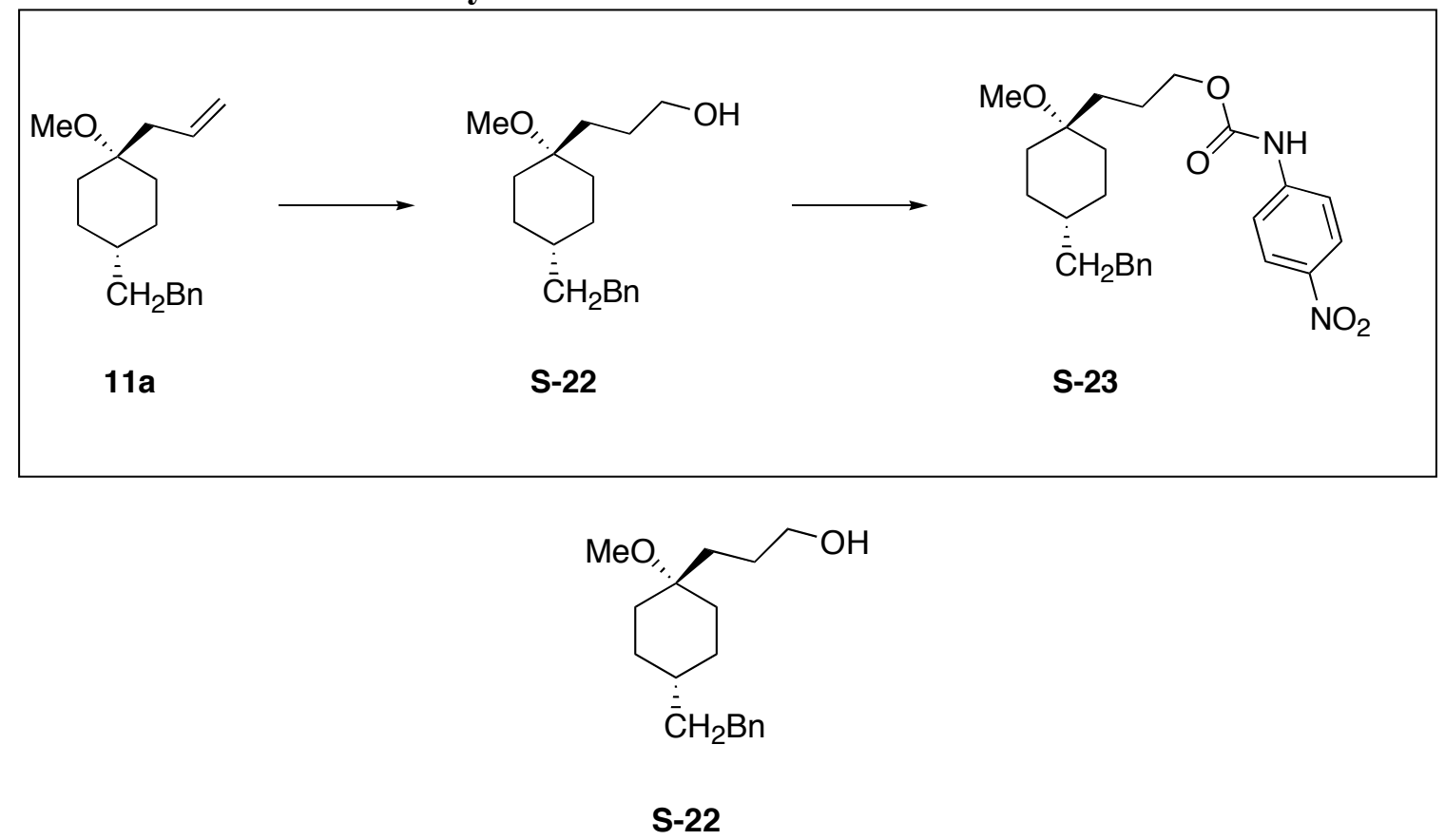

Alcohol S-22: To a solution of 11a $(0.056 \mathrm{~g}, 0.20 \mathrm{mmol})$ in THF $(1 \mathrm{~mL})$ was added a solution of $\mathrm{BH}_{3}$ - THF $(1 \mathrm{M}, 0.40 \mathrm{~mL}, 0.40 \mathrm{mmol})$ and the mixture was stirred for 3 days. A solution of $\mathrm{NaOH}(1 \mathrm{M})$ along with $\mathrm{H}_{2} \mathrm{O}_{2}(30 \%, 1: 1,1 \mathrm{~mL})$ was added and the mixture was stirred for 1 day. The mixture was diluted with $\mathrm{CH}_{2} \mathrm{Cl}_{2}(2 \mathrm{~mL})$. The layers were separated and the aqueous layer was extracted with $\mathrm{CH}_{2} \mathrm{Cl}_{2}(5 \times 2 \mathrm{~mL})$. The organic layers were combined, filtered through $\mathrm{Na}_{2} \mathrm{SO}_{4}$, and concentrated in vacuo. The resulting oil was purified by flash chromatography (94:6 to 83:17 hexanes/EtOAc) to afford the product as a colorless oil $(0.03 \mathrm{~g}, 43 \%)$ : ${ }^{1} \mathrm{H}$ NMR $\left(500 \mathrm{MHz}, \mathrm{CDCl}_{3}\right), \delta$ 7.28$7.25(\mathrm{~m}, 2 \mathrm{H}), 7.18-7.15(\mathrm{~m}, 3 \mathrm{H}), 3.63(\mathrm{t}, J=6.2,2 \mathrm{H}), 3.12(\mathrm{~s}, 3 \mathrm{H}), 2.64-2.61(\mathrm{~m}, 2 \mathrm{H})$, $1.94(\mathrm{br} \mathrm{s}, 1 \mathrm{H}), 1.87(\mathrm{~d}, J=12.4,2 \mathrm{H}), 1.62-1.51(\mathrm{~m}, 5 \mathrm{H}), 1.47(\mathrm{dd}, J=9.8,5.9,2 \mathrm{H})$, 1.25-1.11 (m, 6H); ${ }^{13} \mathrm{C}$ NMR (125 MHz, $\left.\mathrm{CDCl}_{3}\right), \delta$ 143.0, 128.3, 128.2, 125.5, 74.3, 63.4, 48.0, 38.9, 36.8, 33.6, 33.3, 29.7, 27.8, 26.3; IR (thin film) 3062, 2921, 1603, 1455 $\mathrm{cm}^{-1}$; HRMS (EI) $\mathrm{m} / \mathrm{z}$ calcd for $\mathrm{C}_{18} \mathrm{H}_{28} \mathrm{O}_{2} \mathrm{Na}(\mathrm{M}+\mathrm{Na})^{+} 299.1987$, found 299.1978 . 


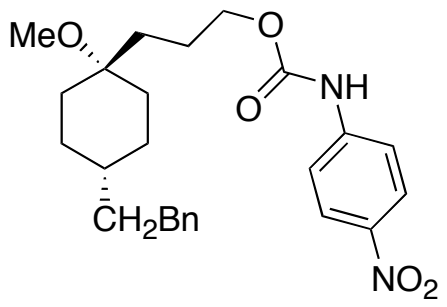

S-23

Carbamate S-23: To a solution of S-22 $(0.026 \mathrm{~g}, 0.094 \mathrm{mmol})$ in THF $(0.5 \mathrm{~mL})$ was added $p$-nitrophenylisocyanate $(0.017 \mathrm{~g}, 0.14 \mathrm{mmol})$ and the mixture was stirred for 5 days. The solvent was removed in vacuo, and the resulting solid was dissolved in $\mathrm{CH}_{2} \mathrm{Cl}_{2}$ $(0.5 \mathrm{~mL})$ and purified by flash chromatography (95:5 to 90:10 hexanes/EtOAc) to afford the product as a colorless solid: (0.02 g, 53\%): $\mathrm{mp} 149{ }^{\circ} \mathrm{C} ;{ }^{1} \mathrm{H}$ NMR (500 MHz, $\left.\mathrm{CDCl}_{3}\right)$, $\delta 8.20(\mathrm{~d}, J=9.1,2 \mathrm{H}) 7.55(\mathrm{~d}, J=9.0,2 \mathrm{H}) 7.29-7.26(\mathrm{~m}, 2 \mathrm{H}), 7.18-7.16(\mathrm{~m}, 3 \mathrm{H}), 6.92$ (br s, $1 \mathrm{H}), 4.21(\mathrm{t}, J=6.6,2 \mathrm{H}), 3.12(\mathrm{~s}, 3 \mathrm{H}), 2.64-2.61(\mathrm{~m}, 2 \mathrm{H}), 1.86(\mathrm{~d}, J=12.4,2 \mathrm{H})$, 1.71 (ddt, $J=11.3,6.7,6.8,2 \mathrm{H}), 1.60-1.52(\mathrm{~m}, 3 \mathrm{H}), 1.48-1.45(\mathrm{~m}, 2 \mathrm{H}), 1.28-1.12(\mathrm{~m}$, $6 \mathrm{H}) ;{ }^{13} \mathrm{C}$ NMR $\left(125 \mathrm{MHz}, \mathrm{CDCl}_{3}\right), \delta 152.9,143.9,143.0,128.3,128.2,125.5,125.2$, 117.7, 74.0, 66.6, 48.0, 38.8, 36.7, 33.6, 33.3, 33.2, 27.8, 22.3; HRMS (EI) $m / z$ calcd for $\mathrm{C}_{25} \mathrm{H}_{32} \mathrm{NaN}_{2} \mathrm{O}_{5}(\mathrm{M}+\mathrm{Na})^{+}$463.2209, found 463.2207. Anal. Calcd for $\mathrm{C}_{25} \mathrm{H}_{32} \mathrm{~N}_{2} \mathrm{O}_{5}$ : C, 68.16; H, 7.32; N, 6.36. Found: C, 68.40; H, 7.39; N, 6.17.

4. Proof of Stereochemistry for $14 \mathrm{~b}$.

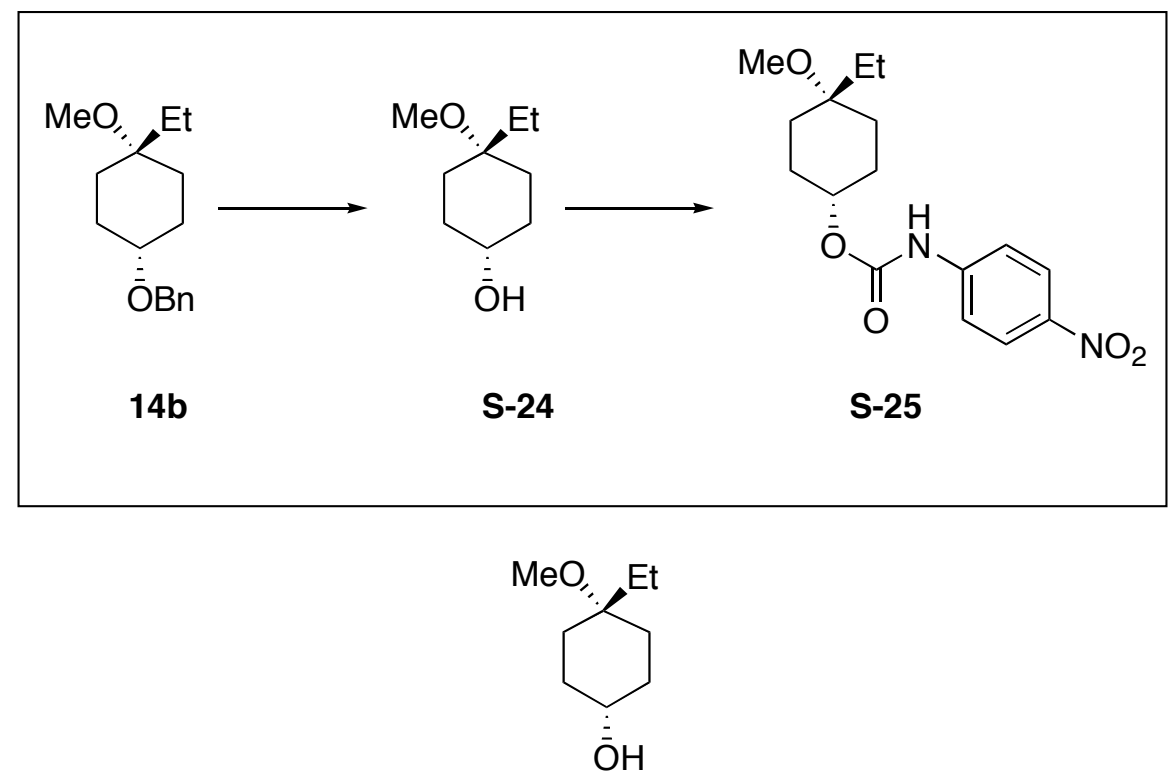

S-24

Alcohol S-24: To a solution of $\mathbf{1 4 b}(0.11 \mathrm{~g}, 0.4 \mathrm{mmol})$ in ethanol $(3 \mathrm{~mL})$ was added $\mathrm{Pd} / \mathrm{C}$ $10 \%,(0.02 \mathrm{~g}, 0.004 \mathrm{mmol})$. The mixture was first purged with $\mathrm{H}_{2}$ then placed under $\mathrm{H}_{2}$ (1 atm) and stirred for 1 day. The mixture was filtered and the solvent removed in vacuo. The resulting oil was purified by flash chromatography (91:9 Hexanes/EtOAc) to afford 
the product as a colorless oil $(0.06 \mathrm{~g}, 80 \%):{ }^{1} \mathrm{H} \mathrm{NMR}\left(500 \mathrm{MHz}, \mathrm{CDCl}_{3}\right) \delta 3.56(\mathrm{tt}, J=$ 10.6, 4.3, 1H), 3.12 (s, 3H), 2.03 (br s, 1H), 1.85 (dtd, $J=14.9,3.7,3.6,2 \mathrm{H}), 1.73$ (ddtd, $J=12.0,4.7,2.1,1.9,2 \mathrm{H}), 1.54$ (dddd, $J=13.1,11.2,10.9,3.6,2 \mathrm{H}), 1.43(\mathrm{q}, J=7.5$, $2 \mathrm{H}), 1.19(\mathrm{td}, J=13.8,3.9,2 \mathrm{H}), 0.83(\mathrm{t}, J=7.5,3 \mathrm{H}) ;{ }^{13} \mathrm{C} \mathrm{NMR}\left(125 \mathrm{MHz}, \mathrm{CDCl}_{3}\right) \delta$ 73.6, 70.4, 47.8, 31.3, 30.5, 28.6, 7.2; IR (thin film) 3381, 2941, 1459, 1366, $1068 \mathrm{~cm}^{-1}$.

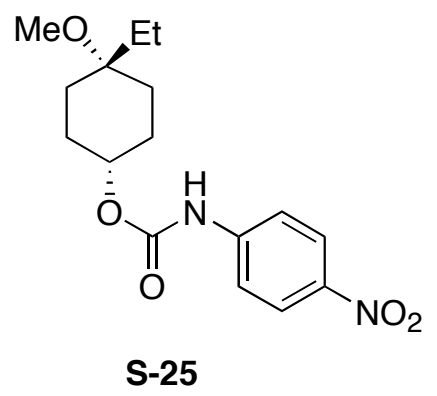

Carbamate S-25: To a solution of S-24 $(0.054 \mathrm{~g}, 0.34 \mathrm{mmol})$ in THF (1 mL) was added $p$-nitrophenylisocyanate $(0.06 \mathrm{~g}, 0.4 \mathrm{mmol})$ and the mixture was stirred for 5 days. The solvent was removed in vacuo, and the resulting solid was dissolved in $\mathrm{CH}_{2} \mathrm{Cl}_{2}(0.50 \mathrm{~mL})$ and purified by flash chromatography (91:9 to 83:17 Hexanes/EtOAc) to afford the product as a colorless solid: (0.091 g, 83\%): mp $176{ }^{\circ} \mathrm{C} ;{ }^{1} \mathrm{H} \mathrm{NMR}\left(500 \mathrm{MHz}, \mathrm{CDCl}_{3}\right) \delta$ $8.19(\mathrm{~d}, J=8.8,2 \mathrm{H}), 7.58(\mathrm{~d}, J=8.7,2 \mathrm{H}), 7.27$ (br s, $1 \mathrm{H}), 4.73(\mathrm{tt}, J=11.1,5.2,1 \mathrm{H})$, 3.15 (s, 3H), 1.93-1.84 (m, 4H), 1.75-1.69 (m, 2H), 1.49 (dd, $J=14.5,7.1,2 \mathrm{H}), 1.33$ (ddd, $J=13.9,13.6,2.7,2 \mathrm{H}), 0.88(\mathrm{t}, J=7.3,3 \mathrm{H}) ;{ }^{13} \mathrm{C} \mathrm{NMR}\left(125 \mathrm{MHz}, \mathrm{CDCl}_{3}\right) \delta 152.5$, 144.4, 142.8, 125.2, 117.6, 74.6, 73.9, 47.8, 31.1, 28.6, 26.8, 7.3; IR (KBr) 3253, 2973, 2877, 1730, 1598, 1223, $1111 \mathrm{~cm}^{-1}$; HRMS (EI) $m / z$ calcd for $\mathrm{C}_{16} \mathrm{H}_{22} \mathrm{O}_{5} \mathrm{~N}_{2} \mathrm{Na}(\mathrm{M}+\mathrm{Na})^{+}$ 345.1426, found 345.1430. Anal. Calcd for $\mathrm{C}_{16} \mathrm{H}_{22} \mathrm{O}_{5} \mathrm{~N}_{2}$ : C, 59.61; H, 6.88; N, 8.69. Found: C, 59.69; H, 6.75; N, 8.53.

\section{Proof of Stereochemistry for $16 b$.}

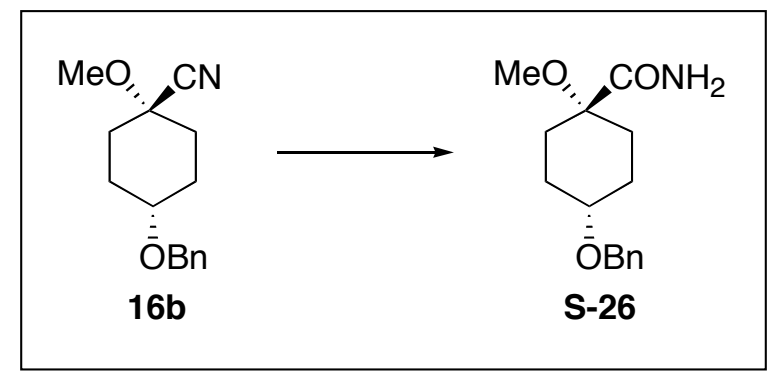

Amide S-26: To a solution of $\mathbf{1 6 b}(0.030 \mathrm{~g}, 0.122 \mathrm{mmol})$ in $\mathrm{EtOH}(1 \mathrm{~mL})$ and $\mathrm{H}_{2} \mathrm{O}_{2}(1$ $\mathrm{mL}, 50 \%)$ was added $\mathrm{K}_{2} \mathrm{CO}_{3}(0.1 \mathrm{~g}, 1 \mathrm{mmol})$. The mixture was allowed to stir for 1 day, then $\mathrm{H}_{2} \mathrm{O}(5 \mathrm{~mL})$ was added along with $\mathrm{CH}_{2} \mathrm{Cl}_{2}(10 \mathrm{~mL})$. The layers were separated and the aqueous layer was extracted with $\mathrm{CH}_{2} \mathrm{Cl}_{2}(5 \times 5 \mathrm{~mL})$. The organic layers were combined, filtered through $\mathrm{Na}_{2} \mathrm{SO}_{4}$, and concentrated in vacuo to afford the product as a colorless solid (0.031 g, 96\%): mp $144{ }^{\circ} \mathrm{C} ;{ }^{1} \mathrm{H} \mathrm{NMR}\left(500 \mathrm{MHz}, \mathrm{CDCl}_{3}\right) \delta$ 7.60-7.31 (m, 4H), 7.29-7.25 (m, 1H), 6.41 (br s, $1 \mathrm{H}), 5.58$ (br s, $1 \mathrm{H}), 4.57(\mathrm{~s}, 2 \mathrm{H}), 3.40$ (tt, $J=10.7$, $4.0,1 \mathrm{H}), 3.26(\mathrm{~s}, 3 \mathrm{H}), 2.01-1.90(\mathrm{~m}, 4 \mathrm{H}), 1.87-1.77(\mathrm{~m}, 2 \mathrm{H}), 1.61-1.52(\mathrm{~m}, 2 \mathrm{H}) ;{ }^{13} \mathrm{C}$ 
NMR $\left(125 \mathrm{MHz}, \mathrm{CDCl}_{3}\right) \delta 177.7,138.8,128.4,127.6,127.5,79.3,76.0,70.0,51.6$, 29.6, 27.1; IR (KBr) 3397, 3197, 2943, 1651, $1103 \mathrm{~cm}^{-1}$; HRMS (ES) $\mathrm{m} / z$ calcd for $\mathrm{C}_{15} \mathrm{H}_{22} \mathrm{O}_{3} \mathrm{~N}(\mathrm{M}+\mathrm{H})^{+} 264.1600$, found 264.1599. Anal. Calcd for $\mathrm{C}_{15} \mathrm{H}_{21} \mathrm{O}_{3} \mathrm{~N}: \mathrm{C}, 68.42$; H, 8.04; N, 5.32. Found: C, 68.63; H, 8.19; N, 5.33.

\section{Proof of Stereochemistry for 17 a.}

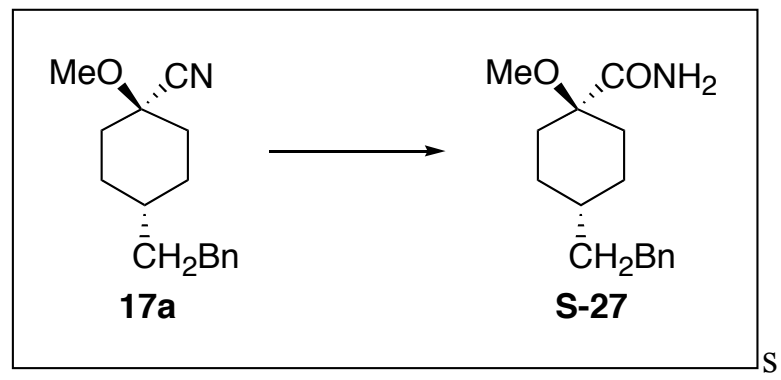

Amide S-27: To a solution of 17a $(0.045 \mathrm{~g}, 0.22 \mathrm{mmol})$ in EtOH $(1.5 \mathrm{~mL})$ and $\mathrm{H}_{2} \mathrm{O}_{2}(1.5$ $\mathrm{mL}, 50 \%)$ was added $\mathrm{K}_{2} \mathrm{CO}_{3}(0.1 \mathrm{~g}, 1 \mathrm{mmol})$. The mixture was allowed to stir for 1 day, then $\mathrm{H}_{2} \mathrm{O}(5 \mathrm{~mL})$ was added along with $\mathrm{CH}_{2} \mathrm{Cl}_{2}(5 \mathrm{~mL})$. The layers were separated and the aqueous layer was extracted with $\mathrm{CH}_{2} \mathrm{Cl}_{2}(5 \times 5 \mathrm{~mL})$. The organic layers were combined, filtered through $\mathrm{Na}_{2} \mathrm{SO}_{4}$, and concentrated in vacuo to afford the product as a colorless solid (0.036 g, 74\%): $\mathrm{mp} 153{ }^{\circ} \mathrm{C} ;{ }^{1} \mathrm{H}$ NMR $\left(500 \mathrm{MHz}, \mathrm{CDCl}_{3}\right)$ \& 7.28-7.25 (m, $2 \mathrm{H}), 7.18-7.15(\mathrm{~m}, 3 \mathrm{H}), 6.28(\mathrm{br} \mathrm{s}, 1 \mathrm{H}), 5.47(\mathrm{br} \mathrm{s}, 1 \mathrm{H}), 2.62-2.59(\mathrm{~m}, 2 \mathrm{H}), 3.25(\mathrm{~s}, 3 \mathrm{H})$, 2.11-2.04 (m, $2 \mathrm{H}), 1.80-1.71(\mathrm{~m}, 2 \mathrm{H}), 1.66-1.61(\mathrm{~m}, 2 \mathrm{H}), 1.55-1.42(\mathrm{~m}, 5 \mathrm{H}) ;{ }^{13} \mathrm{C}$ NMR (125 MHz, $\left.\mathrm{CDCl}_{3}\right), \delta$ 177.4, 142.8, 128.32, 128.26, 125.6, 80.1, 51.4, 35.8, 34.3, 33.8, 30.6, 27.4; IR (KBr) 3372, 2932, 1659, 1449, $1073 \mathrm{~cm}^{-1}$; HRMS (EI) $\mathrm{m} / z$ calcd for $\mathrm{C}_{16} \mathrm{H}_{24} \mathrm{O}_{2} \mathrm{~N}(\mathrm{M}+\mathrm{H})^{+}$262.1807, found 262.1810. Anal. Calcd for $\mathrm{C}_{16} \mathrm{H}_{23} \mathrm{O}_{2} \mathrm{~N}$ : C, 73.53; H, 8.87; N, 5.36. Found: C, 73.67; H, 9.06; N, 5.37.

\section{Proof of Stereochemistry for 24.}

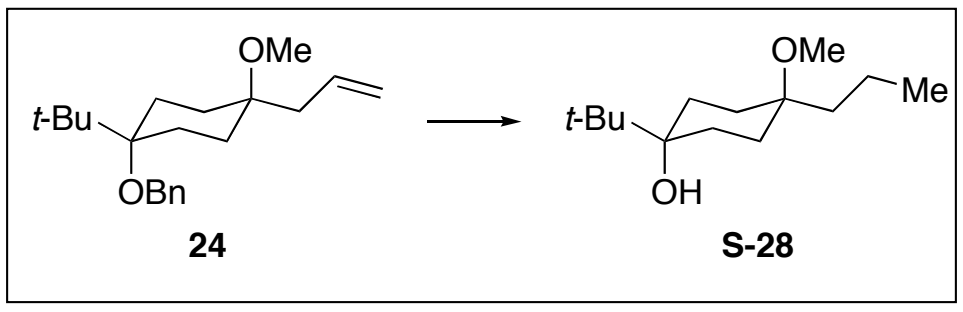

Alcohol S-28: To a solution of $24(0.040 \mathrm{~g}, 0.126 \mathrm{mmol})$ in ethanol $(2 \mathrm{~mL})$ was added $\mathrm{Pd} / \mathrm{C}(10 \%, 0.01 \mathrm{~g}, 0.002 \mathrm{mmol})$. The mixture was first purged with $\mathrm{H}_{2}$ then placed under $\mathrm{H}_{2}(1 \mathrm{~atm})$ and stirred for 1 day. The mixture was filtered and the solvent removed in vacuo to afford the product as colorless solid $(0.02 \mathrm{~g}, 69 \%)$ : mp $42{ }^{\circ} \mathrm{C} ;{ }^{1} \mathrm{H}$ NMR (500 $\left.\mathrm{MHz}, \mathrm{CDCl}_{3}\right) \delta 3.11$ (s, $\left.3 \mathrm{H}\right), 1.76-1.64(\mathrm{~m}, 4 \mathrm{H}), 1.53$ (ddd, $\left.J=13.8,13.7,4.0,2 \mathrm{H}\right), 1.42-$ $1.26(\mathrm{~m}, 7 \mathrm{H}), 0.96-0.90(\mathrm{~m}, 12 \mathrm{H}) ;{ }^{13} \mathrm{C}$ NMR $\left(125 \mathrm{MHz}, \mathrm{CDCl}_{3}\right) \delta$ 74.9, 73.9, 48.3, 39.5, 37.7, 29.2, 26.2, 25.2, 16.0, 14.8; IR (thin film) 3488, 2954, 1637, $1362 \mathrm{~cm}^{-1}$. Anal. Calcd for $\mathrm{C}_{14} \mathrm{H}_{28} \mathrm{O}_{2}$ : C, 73.63; H, 12.36. Found: $\mathrm{C}, 73.75 ; \mathrm{H}, 12.51$. 


\section{Proof of Stereochemistry for 25.}

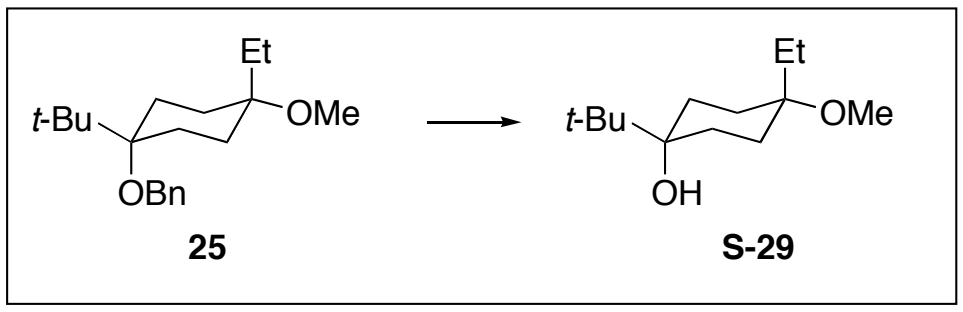

Alcohol S-29: To a solution of $25(0.065 \mathrm{~g}, 0.20 \mathrm{mmol})$ in ethanol $(2 \mathrm{~mL})$ was added $\mathrm{Pd} / \mathrm{C}(10 \%, 0.01 \mathrm{~g}, 0.002 \mathrm{mmol})$. The mixture was first purged with $\mathrm{H}_{2}$ then placed under $\mathrm{H}_{2}(1 \mathrm{~atm})$ and stirred for 1 day. The mixture was filtered and the solvent was removed in vacuo to afford the product as a colorless solid $(0.03 \mathrm{~g}, 72 \%): \mathrm{mp} 38{ }^{\circ} \mathrm{C} ;{ }^{1} \mathrm{H}$ NMR (500 MHz, $\left.\mathrm{CDCl}_{3}\right) \delta 3.18(\mathrm{~s}, 3 \mathrm{H}), 1.72(\mathrm{ddd}, J=13.9,13.5,4.6,2 \mathrm{H}), 1.63-1.57 \mathrm{~m}$, $4 \mathrm{H}), 1.55-1.47(\mathrm{~m}, 4 \mathrm{H}), 1.28(\mathrm{br} \mathrm{s}, 1 \mathrm{H}), 0.94(\mathrm{~s}, 9 \mathrm{H}), 0.84(\mathrm{t}, J=7.4,3 \mathrm{H}) ;{ }^{13} \mathrm{C} \mathrm{NMR}(125$ $\left.\mathrm{MHz}, \mathrm{CDCl}_{3}\right) \delta$ 75.5, 74.3, 48.0, 37.4, 29.2, 28.1, 25.1, 22.7, 6.8; IR (KBr) 3483, 2961, 1464, $1269 \mathrm{~cm}^{-1}$. Anal. Calcd for $\mathrm{C}_{23} \mathrm{H}_{26} \mathrm{O}_{2}$ : C, 72.84; H, 12.22. Found: C, 72.62; $\mathrm{H}$, 12.25 .

\section{Proof of Stereochemistry for 8.}

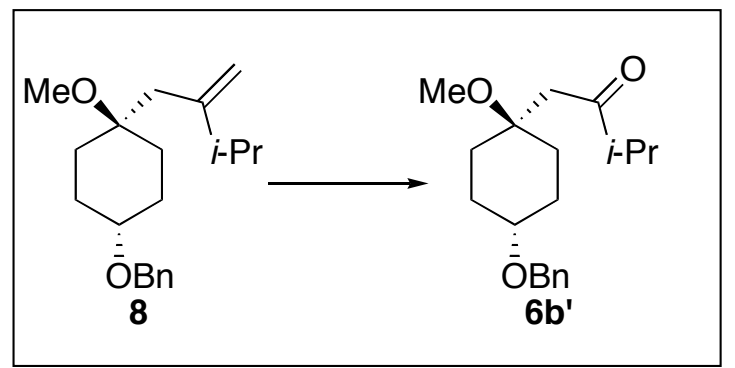

Ketone 6b': To a solution of $8(0.073 \mathrm{~g}, 0.24 \mathrm{mmol})$ in acetone: $\mathrm{H}_{2} \mathrm{O}(5 \mathrm{~mL}, 5: 1)$ was added a solution of OsO4 (4\% wt., $0.02 \mathrm{~mL}, 0.002 \mathrm{mmol})$ and the mixture was stirred for 1 day. A solution of $\mathrm{NaIO}_{4}(0.309 \mathrm{~g}, 1.40 \mathrm{mmol})$ in $\mathrm{H}_{2} \mathrm{O}(2 \mathrm{~mL})$ was added and the mixture was stirred for 4 hours. Then $\mathrm{CH}_{2} \mathrm{Cl}_{2}(5 \mathrm{~mL})$ was added to the mixture and the layers were separated. The aqueous layer was extracted with $\mathrm{CH}_{2} \mathrm{Cl}_{2}(5 \times 5 \mathrm{~mL})$. The organic layers were combined, filtered through $\mathrm{Na}_{2} \mathrm{SO}_{4}$, and concentrated in vacuo. The resulting oil was purified by flash chromatography (91:9 hexanes/ EtOAc) to afford the product as a colorless oil (0.062 g, 84\%). All NMR spectra and GC traces matched to $6 \mathbf{b}$ (major).

\section{Proofs of Stereochemistry for 21 and 22.}

${ }^{1} \mathrm{H}$ NMR studies using nOe's were performed to determine the stereochemistry of $\mathbf{2 1}$ and 22. For alkene 21, proton $b$ was irradiated and proton $a$ showed an enhancement of $8.6 \%$. If the allyl group were axial (as shown in $\mathbf{S - 1 6}$ ), such a large enhancement is unlikely. For bicyclic ether 22, protons $b$ were irradiated and showed a $0.8 \%$ enhancement to proton $a$. If the ethyl group in $\mathbf{2 2}$ was not axial but equatorial (S-18), no enhancement would be observed. 

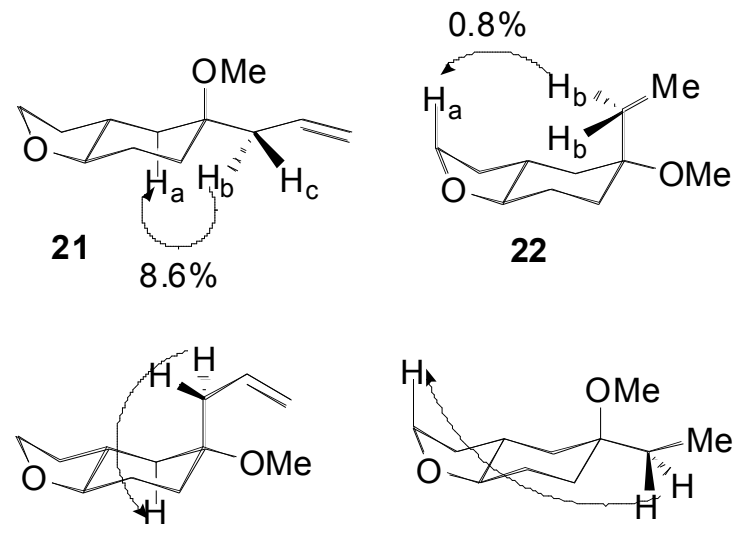

S-16

S-18

\section{References Cited}

1. Pangborn, A. B.; Giardello, M. A.; Grubbs, R. H.; Rosen, R. K., Organometallics 1996, 15, 1518-1520.

2. Chini, M.; Crotti, P.; Flippin, L. A.; Macchia, F., J. Org. Chem. 1990, 55, 4265-4272.

3. Keck, G., E.; Dougherty, S., M.; Savin, K., A., J. Am. Chem. Soc. 1995, 117, 62106223.

4. Napolitano, E.; Fiaschi, R.; Mastrorilli, E., Synthesis 1986, 1, 122-125.

5. Barker, W. R.; Pratt, K. J., Tetrahedron 1993, 49, 8739-8756.

6. Beutelman, H. P.; Xie, L.; Saunders, W. H., J. Org. Chem. 1989, 54, 1703-1709.

\section{Calculations of the Structure of 4-Alkoxy-Substituted Oxocarbenium Ions}

Energy minimization of the cation S-30 at the HF/6-31G* level using MacSpartan (Wavefunction, Irvine, California) showed that the axial conformer is favored by 4.6 $\mathrm{kcal} / \mathrm{mol}$. The structure of the low-energy axial conformer is provided as supporting information.

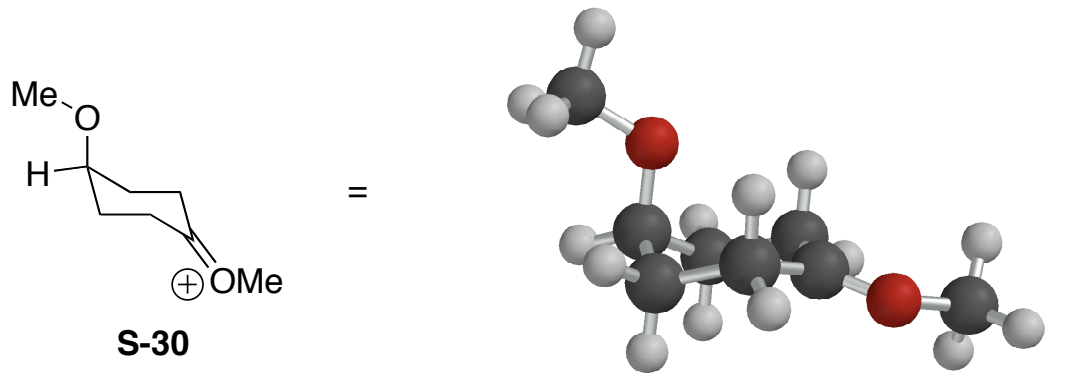

INTERMEDIATE GRADIENT INPUT

Run type: Molecular gradient (no SCF)

Model: RHF/ABASIS

Number of shells: 70

$40 \mathrm{~S}$ shells

20 SP shells

$106 \mathrm{D}$ shells

Number of basis functions: 180 
Number of electrons: $\quad 78$

Number of heavy atoms: $\quad 10$

Number of hydrogens: 15

Use of molecular symmetry disabled

Molecular charge: 1

Spin multiplicity: 1

Memory model: direct $12.1 \mathrm{Mb}$

Point Group = C1

Order $=1$

Nsymop $=1$

This system has 0 degrees of freedom Cartesian gradient (a.u.)

$\begin{array}{ccccc}\text { Atom } & \text { X } & \text { Y } & \text { Z } \\ \text { O } & 1 & -0.0000124 & -0.0000116 & 0.0000076 \\ \text { C } & 2 & 0.0000194 & 0.0000134 & -0.0000106 \\ \text { C } & 3 & -0.0000143 & -0.0000055 & -0.0000281 \\ \text { C } & 4 & 0.0000072 & 0.0000077 & 0.0000089 \\ \text { C } & 5 & -0.0000106 & -0.0000113 & 0.0000138 \\ \text { C } & 6 & -0.0000174 & -0.0000036 & -0.0000050 \\ \text { C } & 7 & 0.0000229 & 0.0000168 & -0.0000193 \\ \text { H } & 8 & 0.0000039 & 0.0000129 & 0.0000045 \\ \text { H } & 9 & 0.0000026 & 0.0000040 & -0.0000090 \\ \text { H } & 10 & -0.0000106 & 0.0000003 & 0.0000042 \\ \text { H } & 11 & 0.0000076 & -0.0000088 & 0.0000066 \\ \text { H } & 12 & -0.0000027 & 0.0000012 & -0.0000054 \\ \text { H } & 13 & -0.0000054 & -0.0000080 & -0.0000055 \\ \text { H } & 14 & -0.0000044 & -0.0000002 & -0.0000002 \\ \text { O } & 15 & 0.0000201 & -0.0000027 & 0.0000021 \\ \text { H } & 16 & 0.0000064 & -0.0000110 & -0.0000003 \\ \text { H } & 17 & -0.0000187 & -0.0000017 & 0.0000316 \\ \text { C } & 18 & 0.0000168 & 0.0000062 & -0.0000040 \\ \text { H } & 19 & -0.0000115 & 0.0000084 & 0.0000036 \\ \text { H } & 20 & -0.0000008 & -0.0000060 & 0.0000085 \\ \text { H } & 21 & 0.0000020 & -0.0000058 & -0.0000087 \\ \text { C } & 22 & -0.0000037 & 0.0000024 & 0.0000041 \\ \text { H } & 23 & -0.0000022 & 0.0000050 & -0.0000062 \\ \text { H } & 24 & 0.0000003 & -0.0000030 & -0.0000005 \\ \text { H } & 25 & 0.0000056 & 0.0000009 & 0.0000075\end{array}$

$\mathrm{E}(\mathrm{HF})=-461.1584552$ a.u. 


\section{X-Ray Crystallographic Data}

1. Carbamate S-20

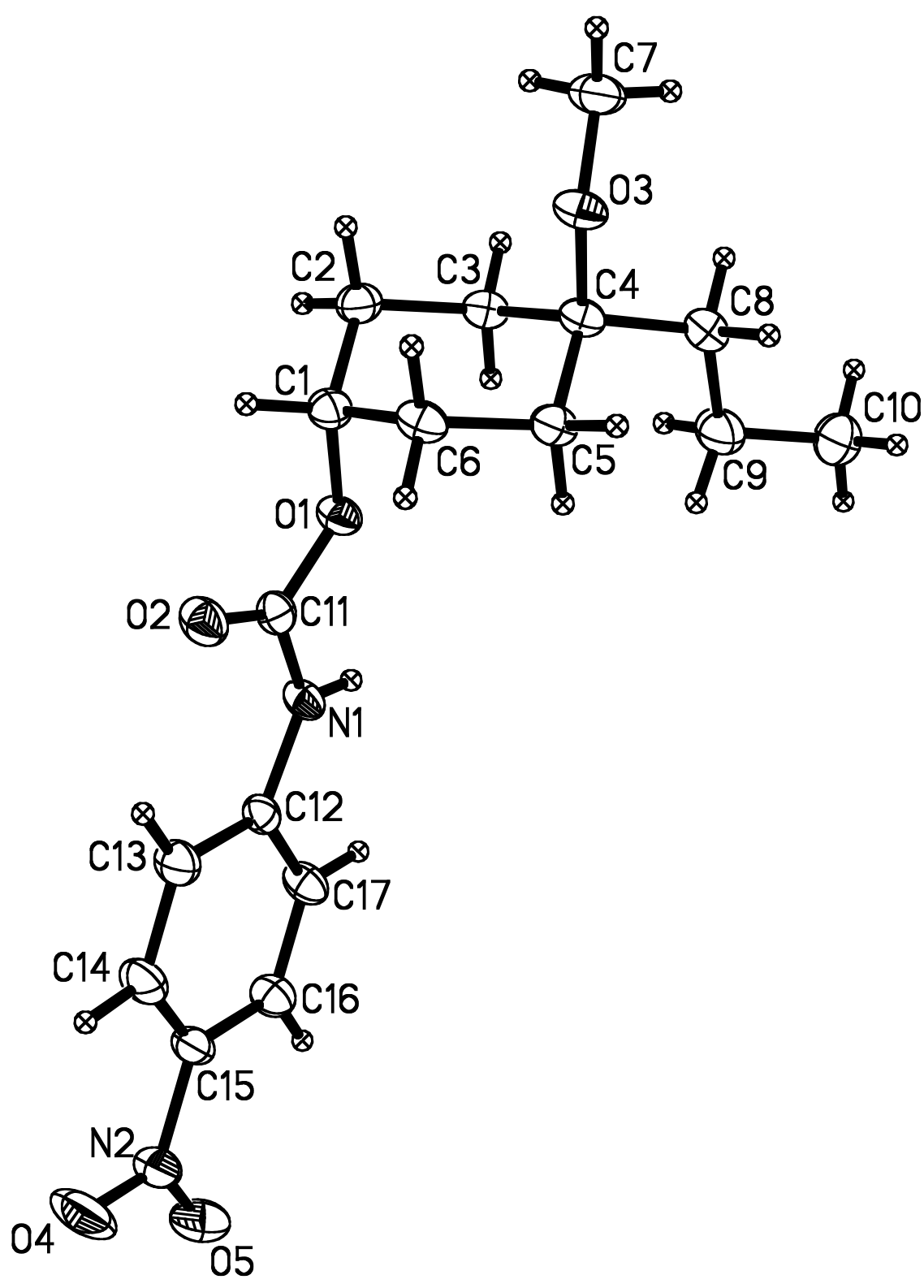

X-ray Data Collection, Structure Solution and Refinement for S-20.

A colorless crystal of approximate dimensions $0.09 \times 0.40 \times 0.47 \mathrm{~mm}$ was mounted on a glass fiber and transferred to a Bruker CCD platform diffractometer. The SMART ${ }^{1}$ program package was used to determine the unit-cell parameters and for data collection 
(25 sec/frame scan time for a sphere of diffraction data). The raw frame data was processed using $\mathrm{SAINT}^{2}$ and $\mathrm{SADABS}^{3}$ to yield the reflection data file. Subsequent calculations were carried out using the SHELXTL ${ }^{4}$ program. The diffraction symmetry was $\mathrm{mmm}$ and the systematic absences were consistent with the orthorhombic space group Pbca which was later determined to be correct.

The structure was solved by direct methods and refined on $\mathrm{F}^{2}$ by full-matrix least-squares techniques. The analytical scattering factors ${ }^{5}$ for neutral atoms were used throughout the analysis. Hydrogen atoms were located from a difference-Fourier map and refined (x,y,z and $\left.U_{\text {iso }}\right)$. At convergence, $w R 2=0.1062$ and $\mathrm{GOF}=1.102$ for 314 variables refined against 4080 data. As a comparison for refinement on F, R1 $=0.0405$ for those 2941 data with $\mathrm{I}>2.0 \sigma(\mathrm{I})$.

Definitions:

$\mathrm{wR} 2=\left[\Sigma\left[\mathrm{w}\left(\mathrm{F}_{\mathrm{o}}^{2}-\mathrm{F}_{\mathrm{c}}^{2}\right)^{2}\right] / \Sigma\left[\mathrm{w}\left(\mathrm{F}_{\mathrm{o}}^{2}\right)^{2}\right]\right]^{1 / 2}$

$\mathrm{R} 1=\Sigma|| \mathrm{F}_{\mathrm{o}}|-| \mathrm{F}_{\mathrm{c}}|| / \Sigma\left|\mathrm{F}_{\mathrm{o}}\right|$

Goof $=\mathrm{S}=\left[\Sigma\left[\mathrm{w}\left(\mathrm{F}_{\mathrm{o}}{ }^{2}-\mathrm{F}_{\mathrm{c}}{ }^{2}\right)^{2}\right] /(\mathrm{n}-\mathrm{p})\right]^{1 / 2}$ where $\mathrm{n}$ is the number of reflections and $\mathrm{p}$ is the total

number of parameters refined.

The thermal ellipsoid plot is shown at the 50\% probability level.

Table 1. Crystal data and structure refinement for $\mathbf{S - 2 0 .}$

Identification code

Empirical formula

Formula weight

Temperature

Wavelength

Crystal system

Space group

Unit cell dimensions

Volume

Z

Density (calculated)

Absorption coefficient

$\mathrm{F}(000)$

Crystal color
S-20 (Glen Baghdasarian)

$\mathrm{C}_{17} \mathrm{H}_{24} \mathrm{~N}_{2} \mathrm{O}_{5}$

336.38

163(2) K

$0.71073 \AA$

Orthorhombic

$\mathrm{Pbca}$

$a=15.4190(9) \AA \quad \alpha=90^{\circ}$.

$\mathrm{b}=7.0236(4) \AA \quad \beta=90^{\circ}$.

$\mathrm{c}=30.8348(18) \AA \quad \gamma=90^{\circ}$.

3339.3(3) $\AA^{3}$

8

$1.338 \mathrm{Mg} / \mathrm{m}^{3}$

$0.099 \mathrm{~mm}^{-1}$

1440

colorless 
Crystal size

Theta range for data collection

Index ranges

Reflections collected

Independent reflections

Completeness to theta $=28.30^{\circ}$

Absorption correction

Max. and min. transmission

Refinement method

Data / restraints / parameters

Goodness-of-fit on $\mathrm{F}^{2}$

Final $R$ indices $[\mathrm{I}>2 \operatorname{sigma}(\mathrm{I})=2941$ data $]$

$\mathrm{R}$ indices (all data)

Extinction coefficient

Largest diff. peak and hole
$0.47 \times 0.40 \times 0.09 \mathrm{~mm}^{3}$

1.32 to $28.30^{\circ}$.

$-20 \leq h \leq 20,-9 \leq k \leq 9,-40 \leq l \leq 40$

32020

$4080[\mathrm{R}(\mathrm{int})=0.0560]$

$98.3 \%$

Semi-empirical from equivalents

0.9912 and 0.9551

Full-matrix least-squares on $\mathrm{F}^{2}$

4080 / 0 / 314

1.012

$\mathrm{R} 1=0.0405, \mathrm{wR} 2=0.0922$

$\mathrm{R} 1=0.0680, \mathrm{wR} 2=0.1062$

$0.0018(4)$

0.278 and -0.189 e. $\AA^{-3}$

Table 2. Atomic coordinates ( $\mathrm{x} 10^{4}$ ) and equivalent isotropic displacement parameters $\left(\AA^{2} \times 10^{3}\right)$

for S-20. U(eq) is defined as one third of the trace of the orthogonalized $U^{i j}$ tensor.

\begin{tabular}{lrrrr}
\hline & & & \\
& $\mathrm{x}$ & $\mathrm{y}$ & $\mathrm{U}(\mathrm{eq})$ \\
& & & & \\
\hline $\mathrm{O}(1)$ & & & & \\
$\mathrm{O}(2)$ & $1154(1)$ & $251(1)$ & $6338(1)$ & $24(1)$ \\
$\mathrm{O}(3)$ & $2586(1)$ & $-14(2)$ & $6500(1)$ & $30(1)$ \\
$\mathrm{O}(4)$ & $-885(1)$ & $1746(2)$ & $7323(1)$ & $27(1)$ \\
$\mathrm{O}(5)$ & $5842(1)$ & $2540(2)$ & $5104(1)$ & $56(1)$ \\
$\mathrm{N}(1)$ & $5092(1)$ & $3405(2)$ & $4553(1)$ & $41(1)$ \\
$\mathrm{N}(2)$ & $2103(1)$ & $979(2)$ & $5831(1)$ & $22(1)$ \\
$\mathrm{C}(1)$ & $5144(1)$ & $2762(2)$ & $4921(1)$ & $32(1)$ \\
$\mathrm{C}(2)$ & $917(1)$ & $-233(2)$ & $6787(1)$ & $24(1)$ \\
$\mathrm{C}(3)$ & $19(1)$ & $-1111(2)$ & $6762(1)$ & $26(1)$ \\
$\mathrm{C}(4)$ & $-657(1)$ & $317(2)$ & $6603(1)$ & $23(1)$ \\
$\mathrm{C}(5)$ & $-676(1)$ & $2148(2)$ & $6873(1)$ & $23(1)$ \\
& $240(1)$ & $2992(2)$ & $6898(1)$ & $24(1)$
\end{tabular}




$\begin{array}{lrrrr}\mathrm{C}(6) & 920(1) & 1568(2) & 7056(1) & 25(1) \\ \mathrm{C}(7) & -1689(1) & 841(3) & 7408(1) & 34(1) \\ \mathrm{C}(8) & -1326(1) & 3610(2) & 6695(1) & 26(1) \\ \mathrm{C}(9) & -1205(1) & 4188(3) & 6226(1) & 39(1) \\ \mathrm{C}(10) & -1846(1) & 5653(3) & 6068(1) & 39(1) \\ \mathrm{C}(11) & 2006(1) & 369(2) & 6251(1) & 22(1) \\ \mathrm{C}(12) & 2879(1) & 1337(2) & 5614(1) & 20(1) \\ \mathrm{C}(13) & 3695(1) & 1068(2) & 5801(1) & 29(1) \\ \mathrm{C}(14) & 4429(1) & 1542(2) & 5569(1) & 32(1) \\ \mathrm{C}(15) & 4359(1) & 2255(2) & 5155(1) & 25(1) \\ \mathrm{C}(16) & 3558(1) & 2506(2) & 4959(1) & 26(1) \\ \mathrm{C}(17) & 2823(1) & 2038(2) & 5189(1) & 26(1)\end{array}$

Table 3. Bond lengths $[\AA]$ and angles $\left[^{\circ}\right]$ for $\mathbf{S - 2 0}$.

\begin{tabular}{ll}
\hline $\mathrm{O}(1)-\mathrm{C}(11)$ & $1.3431(16)$ \\
$\mathrm{O}(1)-\mathrm{C}(1)$ & $1.4710(16)$ \\
$\mathrm{O}(2)-\mathrm{C}(11)$ & $1.2069(17)$ \\
$\mathrm{O}(3)-\mathrm{C}(7)$ & $1.4184(19)$ \\
$\mathrm{O}(3)-\mathrm{C}(4)$ & $1.4508(16)$ \\
$\mathrm{O}(4)-\mathrm{N}(2)$ & $1.2256(17)$ \\
$\mathrm{O}(5)-\mathrm{N}(2)$ & $1.2243(18)$ \\
$\mathrm{N}(1)-\mathrm{C}(11)$ & $1.3739(18)$ \\
$\mathrm{N}(1)-\mathrm{C}(12)$ & $1.3949(17)$ \\
$\mathrm{N}(2)-\mathrm{C}(15)$ & $1.4529(18)$ \\
$\mathrm{C}(1)-\mathrm{C}(6)$ & $1.514(2)$ \\
$\mathrm{C}(1)-\mathrm{C}(2)$ & $1.517(2)$ \\
$\mathrm{C}(2)-\mathrm{C}(3)$ & $1.528(2)$ \\
$\mathrm{C}(3)-\mathrm{C}(4)$ & $1.533(2)$ \\
$\mathrm{C}(4)-\mathrm{C}(5)$ & $1.5335(19)$ \\
$\mathrm{C}(4)-\mathrm{C}(8)$ & $1.536(2)$ \\
$\mathrm{C}(5)-\mathrm{C}(6)$ & $1.529(2)$ \\
$\mathrm{C}(8)-\mathrm{C}(9)$ & $1.513(2)$ \\
$\mathrm{C}(9)-\mathrm{C}(10)$ & $1.508(2)$
\end{tabular}




$\begin{array}{lr}\mathrm{C}(12)-\mathrm{C}(13) & 1.3961(19) \\ \mathrm{C}(12)-\mathrm{C}(17) & 1.401(2) \\ \mathrm{C}(13)-\mathrm{C}(14) & 1.379(2) \\ \mathrm{C}(14)-\mathrm{C}(15) & 1.377(2) \\ \mathrm{C}(15)-\mathrm{C}(16) & 1.385(2) \\ \mathrm{C}(16)-\mathrm{C}(17) & 1.377(2) \\ \mathrm{C}(11)-\mathrm{O}(1)-\mathrm{C}(1) & 116.41(11) \\ \mathrm{C}(7)-\mathrm{O}(3)-\mathrm{C}(4) & 117.26(11) \\ \mathrm{C}(11)-\mathrm{N}(1)-\mathrm{C}(12) & 127.04(12) \\ \mathrm{O}(5)-\mathrm{N}(2)-\mathrm{O}(4) & 122.09(13) \\ \mathrm{O}(5)-\mathrm{N}(2)-\mathrm{C}(15) & 119.70(13) \\ \mathrm{O}(4)-\mathrm{N}(2)-\mathrm{C}(15) & 118.21(14) \\ \mathrm{O}(1)-\mathrm{C}(1)-\mathrm{C}(6) & 108.83(12) \\ \mathrm{O}(1)-\mathrm{C}(1)-\mathrm{C}(2) & 105.95(11) \\ \mathrm{C}(6)-\mathrm{C}(1)-\mathrm{C}(2) & 111.71(12) \\ \mathrm{C}(1)-\mathrm{C}(2)-\mathrm{C}(3) & 111.80(12) \\ \mathrm{C}(2)-\mathrm{C}(3)-\mathrm{C}(4) & 112.88(12) \\ \mathrm{O}(3)-\mathrm{C}(4)-\mathrm{C}(3) & 111.14(12) \\ \mathrm{O}(3)-\mathrm{C}(4)-\mathrm{C}(5) & 103.33(11) \\ \mathrm{C}(3)-\mathrm{C}(4)-\mathrm{C}(5) & 109.51(11) \\ \mathrm{O}(3)-\mathrm{C}(4)-\mathrm{C}(8) & 109.17(11) \\ \mathrm{C}(3)-\mathrm{C}(4)-\mathrm{C}(8) & 112.24(12) \\ \mathrm{C}(5)-\mathrm{C}(4)-\mathrm{C}(8) & 111.11(12) \\ \mathrm{C}(6)-\mathrm{C}(5)-\mathrm{C}(4) & 113.29(12) \\ \mathrm{C}(1)-\mathrm{C}(6)-\mathrm{C}(5) & 111.65(12) \\ \mathrm{C}(9)-\mathrm{C}(8)-\mathrm{C}(4) & 116.20(12) \\ \mathrm{C}(10)-\mathrm{C}(9)-\mathrm{C}(8) & 114.27(15) \\ \mathrm{O}(2)-\mathrm{C}(11)-\mathrm{O}(1) & 125.75(13) \\ \mathrm{O}(2)-\mathrm{C}(11)-\mathrm{N}(1) & 126.02(13) \\ \mathrm{O}(1)-\mathrm{C}(11)-\mathrm{N}(1) & 108.23(11) \\ \mathrm{N}(1)-\mathrm{C}(12)-\mathrm{C}(13) & 123.37(13) \\ \mathrm{N}(1)-\mathrm{C}(12)-\mathrm{C}(17) & 117.31(12) \\ \mathrm{C}(13)-\mathrm{C}(12)-\mathrm{C}(17) & 119.31(13) \\ \mathrm{C}(14)-\mathrm{C}(13)-\mathrm{C}(12) & 119.49(14) \\ \mathrm{C}(15)-\mathrm{C}(14)-\mathrm{C}(13) & 120.29(14) \\ \mathrm{C}(14)-\mathrm{C}(15)-\mathrm{C}(16) & 121.30(13)\end{array}$




$\begin{array}{ll}\mathrm{C}(14)-\mathrm{C}(15)-\mathrm{N}(2) & 118.96(13) \\ \mathrm{C}(16)-\mathrm{C}(15)-\mathrm{N}(2) & 119.74(13) \\ \mathrm{C}(17)-\mathrm{C}(16)-\mathrm{C}(15) & 118.68(14) \\ \mathrm{C}(16)-\mathrm{C}(17)-\mathrm{C}(12) & 120.91(13)\end{array}$

Table 4. Anisotropic displacement parameters $\left(\AA^{2} \times 10^{3}\right)$ for S-20. The anisotropic displacement factor exponent takes the form: $-2 \pi^{2}\left[h^{2} a^{* 2} U^{11}+\ldots+2 h k a^{*} b^{*} U^{12}\right]$

\begin{tabular}{|c|c|c|c|c|c|c|}
\hline & $\mathrm{U}^{11}$ & $\mathrm{U}^{22}$ & $\mathrm{U}^{33}$ & $\mathrm{U}^{23}$ & $U^{13}$ & $\mathrm{U}^{12}$ \\
\hline $\mathrm{O}(1)$ & 21(1) & $31(1)$ & $20(1)$ & 1(1) & $3(1)$ & $0(1)$ \\
\hline $\mathrm{O}(2)$ & $24(1)$ & $41(1)$ & $26(1)$ & $5(1)$ & $-1(1)$ & $5(1)$ \\
\hline $\mathrm{O}(3)$ & $24(1)$ & $37(1)$ & $20(1)$ & $0(1)$ & $5(1)$ & $-4(1)$ \\
\hline $\mathrm{O}(4)$ & $18(1)$ & $83(1)$ & $68(1)$ & $17(1)$ & $5(1)$ & $-2(1)$ \\
\hline $\mathrm{O}(5)$ & $37(1)$ & $49(1)$ & $37(1)$ & $4(1)$ & $13(1)$ & $-7(1)$ \\
\hline $\mathrm{N}(1)$ & $16(1)$ & $30(1)$ & $21(1)$ & $0(1)$ & $-1(1)$ & $2(1)$ \\
\hline $\mathrm{N}(2)$ & $23(1)$ & $32(1)$ & $42(1)$ & $-1(1)$ & $8(1)$ & $-2(1)$ \\
\hline $\mathrm{C}(1)$ & $25(1)$ & $28(1)$ & $20(1)$ & $4(1)$ & $3(1)$ & 2(1) \\
\hline$C(2)$ & $29(1)$ & $24(1)$ & $24(1)$ & $1(1)$ & $4(1)$ & $-2(1)$ \\
\hline$C(3)$ & $22(1)$ & $27(1)$ & $21(1)$ & $-1(1)$ & $2(1)$ & $-4(1)$ \\
\hline $\mathrm{C}(4)$ & $21(1)$ & $27(1)$ & $19(1)$ & $-1(1)$ & $3(1)$ & $-2(1)$ \\
\hline$C(5)$ & $23(1)$ & $26(1)$ & $24(1)$ & $-3(1)$ & $3(1)$ & $-3(1)$ \\
\hline$C(6)$ & 21(1) & $34(1)$ & $20(1)$ & $-1(1)$ & 1(1) & $-3(1)$ \\
\hline$C(7)$ & $26(1)$ & $45(1)$ & $31(1)$ & $10(1)$ & $6(1)$ & $-2(1)$ \\
\hline $\mathrm{C}(8)$ & $23(1)$ & $29(1)$ & $26(1)$ & $-2(1)$ & $3(1)$ & $1(1)$ \\
\hline $\mathrm{C}(9)$ & $35(1)$ & $47(1)$ & $35(1)$ & $14(1)$ & $9(1)$ & $10(1)$ \\
\hline$C(10)$ & $38(1)$ & $38(1)$ & $40(1)$ & $7(1)$ & $0(1)$ & $7(1)$ \\
\hline $\mathrm{C}(11)$ & $23(1)$ & 21(1) & $23(1)$ & $-3(1)$ & 1(1) & $2(1)$ \\
\hline$C(12)$ & $19(1)$ & $20(1)$ & $22(1)$ & $-4(1)$ & $2(1)$ & $1(1)$ \\
\hline$C(13)$ & $23(1)$ & $37(1)$ & $28(1)$ & $7(1)$ & $-1(1)$ & $3(1)$ \\
\hline$C(14)$ & $17(1)$ & $42(1)$ & $38(1)$ & $7(1)$ & $-3(1)$ & $1(1)$ \\
\hline$C(15)$ & $19(1)$ & $27(1)$ & $30(1)$ & $-2(1)$ & $5(1)$ & $-1(1)$ \\
\hline$C(16)$ & $25(1)$ & $33(1)$ & $21(1)$ & $0(1)$ & $1(1)$ & $0(1)$ \\
\hline
\end{tabular}


Table 5. Hydrogen coordinates ( x 104) and isotropic displacement parameters $\left(\AA^{2} \mathrm{X} 10^{3}\right)$

for S-20.

\begin{tabular}{|c|c|c|c|c|}
\hline & $\mathrm{x}$ & $\mathrm{y}$ & $\mathrm{z}$ & $\mathrm{U}(\mathrm{eq})$ \\
\hline $\mathrm{H}(1)$ & $1650(11)$ & $1170(20)$ & $5700(5)$ & $23(4)$ \\
\hline $\mathrm{H}(1 \mathrm{~A})$ & $1342(10)$ & $-1130(20)$ & $6900(5)$ & $18(4)$ \\
\hline $\mathrm{H}(2 \mathrm{~A})$ & $-128(9)$ & $-1560(20)$ & $7061(5)$ & $22(4)$ \\
\hline $\mathrm{H}(2 \mathrm{~B})$ & $35(10)$ & $-2260(20)$ & $6565(5)$ & $30(4)$ \\
\hline $\mathrm{H}(3 \mathrm{~A})$ & $-513(9)$ & $650(20)$ & $6308(5)$ & $21(4)$ \\
\hline $\mathrm{H}(3 \mathrm{~B})$ & $-1235(11)$ & $-280(20)$ & $6595(5)$ & $30(4)$ \\
\hline $\mathrm{H}(5 \mathrm{~A})$ & 213(9) & $4090(20)$ & $7090(5)$ & $23(4)$ \\
\hline $\mathrm{H}(5 \mathrm{~B})$ & $411(10)$ & $3450(20)$ & $6594(5)$ & $25(4)$ \\
\hline $\mathrm{H}(6 \mathrm{~A})$ & 794(10) & $1200(20)$ & $7360(6)$ & $30(4)$ \\
\hline $\mathrm{H}(6 \mathrm{~B})$ & 1491(10) & $2160(20)$ & $7050(5)$ & $24(4)$ \\
\hline $\mathrm{H}(7 \mathrm{~A})$ & $-1731(10)$ & $720(20)$ & $7724(6)$ & $31(4)$ \\
\hline $\mathrm{H}(7 \mathrm{~B})$ & $-2167(13)$ & $1540(30)$ & $7300(6)$ & $52(6)$ \\
\hline $\mathrm{H}(7 \mathrm{C})$ & $-1706(13)$ & $-450(30)$ & $7269(7)$ & $57(6)$ \\
\hline $\mathrm{H}(8 \mathrm{~A})$ & $-1302(12)$ & $4760(30)$ & $6885(6)$ & $42(5)$ \\
\hline $\mathrm{H}(8 \mathrm{~B})$ & $-1908(11)$ & $3100(20)$ & $6724(5)$ & $34(5)$ \\
\hline $\mathrm{H}(9 \mathrm{~A})$ & $-633(16)$ & $4560(30)$ & $6171(8)$ & $72(7)$ \\
\hline $\mathrm{H}(9 \mathrm{~B})$ & $-1240(15)$ & $3070(40)$ & $6043(8)$ & $74(8)$ \\
\hline $\mathrm{H}(10 \mathrm{~A})$ & $-1759(12)$ & $6020(30)$ & $5769(7)$ & $45(5)$ \\
\hline $\mathrm{H}(10 \mathrm{~B})$ & $-1834(15)$ & $6730(40)$ & $6248(8)$ & $71(7)$ \\
\hline $\mathrm{H}(10 \mathrm{C})$ & $-2432(16)$ & $5150(30)$ & $6092(7)$ & $64(6)$ \\
\hline $\mathrm{H}(13 \mathrm{~A})$ & $3736(11)$ & $580(30)$ & $6086(6)$ & $40(5)$ \\
\hline $\mathrm{H}(14 \mathrm{~A})$ & $4997(12)$ & $1420(30)$ & $5688(6)$ & $44(5)$ \\
\hline $\mathrm{H}(16 \mathrm{~A})$ & $3529(10)$ & $3000(20)$ & $4668(6)$ & $34(5)$ \\
\hline
\end{tabular}


Table 6. Torsion angles $\left[^{\circ}\right]$ for $\mathbf{S - 2 0 .}$

\begin{tabular}{|c|c|}
\hline $\mathrm{C}(11)-\mathrm{O}(1)-\mathrm{C}(1)-\mathrm{C}(6)$ & $83.97(14)$ \\
\hline $\mathrm{C}(11)-\mathrm{O}(1)-\mathrm{C}(1)-\mathrm{C}(2)$ & $-155.78(12)$ \\
\hline $\mathrm{O}(1)-\mathrm{C}(1)-\mathrm{C}(2)-\mathrm{C}(3)$ & $-64.32(15)$ \\
\hline$C(6)-C(1)-C(2)-C(3)$ & $54.05(16)$ \\
\hline$C(1)-C(2)-C(3)-C(4)$ & $-54.68(16)$ \\
\hline $\mathrm{C}(7)-\mathrm{O}(3)-\mathrm{C}(4)-\mathrm{C}(3)$ & $-59.28(16)$ \\
\hline $\mathrm{C}(7)-\mathrm{O}(3)-\mathrm{C}(4)-\mathrm{C}(5)$ & $-176.65(13)$ \\
\hline $\mathrm{C}(7)-\mathrm{O}(3)-\mathrm{C}(4)-\mathrm{C}(8)$ & $65.05(16)$ \\
\hline $\mathrm{C}(2)-\mathrm{C}(3)-\mathrm{C}(4)-\mathrm{O}(3)$ & $-60.38(15)$ \\
\hline$C(2)-C(3)-C(4)-C(5)$ & $53.15(16)$ \\
\hline $\mathrm{C}(2)-\mathrm{C}(3)-\mathrm{C}(4)-\mathrm{C}(8)$ & $177.05(12)$ \\
\hline $\mathrm{O}(3)-\mathrm{C}(4)-\mathrm{C}(5)-\mathrm{C}(6)$ & $65.47(14)$ \\
\hline$C(3)-C(4)-C(5)-C(6)$ & $-53.03(15)$ \\
\hline$C(8)-C(4)-C(5)-C(6)$ & $-177.59(12)$ \\
\hline $\mathrm{O}(1)-\mathrm{C}(1)-\mathrm{C}(6)-\mathrm{C}(5)$ & $63.02(15)$ \\
\hline$C(2)-C(1)-C(6)-C(5)$ & $-53.61(16)$ \\
\hline $\mathrm{C}(4)-\mathrm{C}(5)-\mathrm{C}(6)-\mathrm{C}(1)$ & $54.16(16)$ \\
\hline $\mathrm{O}(3)-\mathrm{C}(4)-\mathrm{C}(8)-\mathrm{C}(9)$ & $-179.73(14)$ \\
\hline$C(3)-C(4)-C(8)-C(9)$ & $-56.05(18)$ \\
\hline$C(5)-C(4)-C(8)-C(9)$ & $66.95(18)$ \\
\hline$C(4)-C(8)-C(9)-C(10)$ & $-178.89(16)$ \\
\hline $\mathrm{C}(1)-\mathrm{O}(1)-\mathrm{C}(11)-\mathrm{O}(2)$ & $5.5(2)$ \\
\hline $\mathrm{C}(1)-\mathrm{O}(1)-\mathrm{C}(11)-\mathrm{N}(1)$ & $-175.18(11)$ \\
\hline $\mathrm{C}(12)-\mathrm{N}(1)-\mathrm{C}(11)-\mathrm{O}(2)$ & $-2.4(2)$ \\
\hline $\mathrm{C}(12)-\mathrm{N}(1)-\mathrm{C}(11)-\mathrm{O}(1)$ & $178.30(13)$ \\
\hline $\mathrm{C}(11)-\mathrm{N}(1)-\mathrm{C}(12)-\mathrm{C}(13)$ & $1.6(2)$ \\
\hline $\mathrm{C}(11)-\mathrm{N}(1)-\mathrm{C}(12)-\mathrm{C}(17)$ & $-177.14(14)$ \\
\hline $\mathrm{N}(1)-\mathrm{C}(12)-\mathrm{C}(13)-\mathrm{C}(14)$ & $-177.01(15)$ \\
\hline$C(17)-C(12)-C(13)-C(14)$ & $1.7(2)$ \\
\hline$C(12)-C(13)-C(14)-C(15)$ & $-0.7(3)$ \\
\hline
\end{tabular}



$\mathrm{C}(13)-\mathrm{C}(14)-\mathrm{C}(15)-\mathrm{C}(16)$
$-0.4(3)$
$\mathrm{C}(13)-\mathrm{C}(14)-\mathrm{C}(15)-\mathrm{N}(2)$
$179.26(15)$
$\mathrm{O}(5)-\mathrm{N}(2)-\mathrm{C}(15)-\mathrm{C}(14)$
$-179.75(15)$
$\mathrm{O}(4)-\mathrm{N}(2)-\mathrm{C}(15)-\mathrm{C}(14)$
$-0.3(2)$
$\mathrm{O}(5)-\mathrm{N}(2)-\mathrm{C}(15)-\mathrm{C}(16)$
$\mathrm{O}(4)-\mathrm{N}(2)-\mathrm{C}(15)-\mathrm{C}(16)$
$179.37(15)$
$\mathrm{C}(14)-\mathrm{C}(15)-\mathrm{C}(16)-\mathrm{C}(17)$
$0.4(2)$
$\mathrm{N}(2)-\mathrm{C}(15)-\mathrm{C}(16)-\mathrm{C}(17)$
$-179.21(14)$
$\mathrm{C}(15)-\mathrm{C}(16)-\mathrm{C}(17)-\mathrm{C}(12)$
$0.6(2)$
$\mathrm{N}(1)-\mathrm{C}(12)-\mathrm{C}(17)-\mathrm{C}(16)$
$177.13(14)$

\section{Hydrazone S-21}

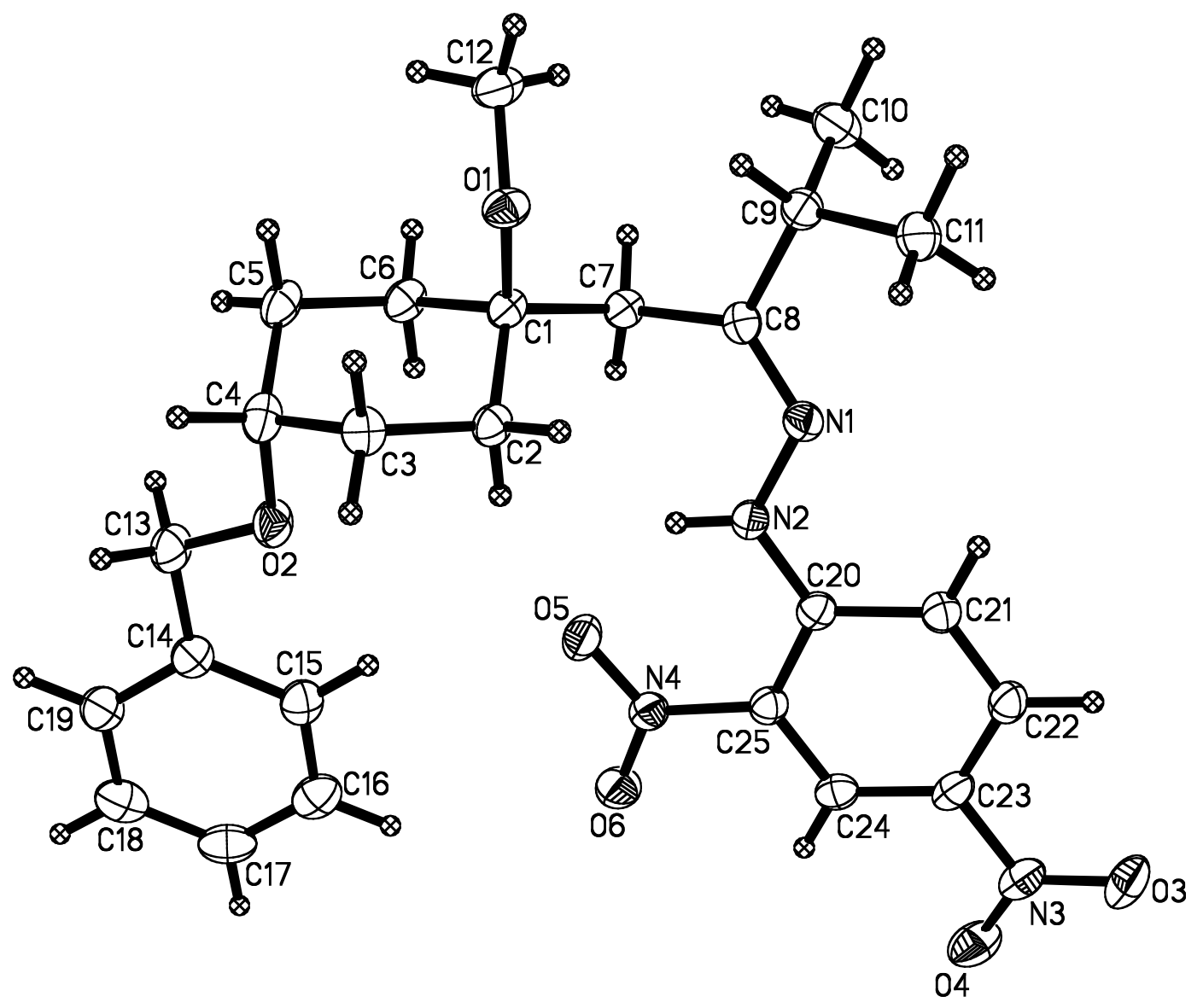

X-ray Data Collection, Structure Solution and Refinement for S-21. 
A yellow crystal of approximate dimensions $0.19 \times 0.27 \times 0.35 \mathrm{~mm}$ was mounted on a glass fiber and transferred to a Bruker CCD platform diffractometer. The SMART ${ }^{1}$ program package was used to determine the unit-cell parameters and for data collection (25 sec/frame scan time for a sphere of diffraction data). The raw frame data was processed using $\mathrm{SAINT}^{2}$ and $\mathrm{SADABS}^{3}$ to yield the reflection data file. Subsequent calculations were carried out using the SHELXTL ${ }^{4}$ program. There were no systematic absences nor any diffraction symmetry other than the Friedel condition. The centrosymmetric triclinic space group $\mathrm{P} \overline{1}$ was assigned and later determined to be correct.

The structure was solved by direct methods and refined on $\mathrm{F}^{2}$ by full-matrix least-squares techniques. The analytical scattering factors ${ }^{5}$ for neutral atoms were used throughout the analysis. Hydrogen atoms were located from a difference-Fourier map and refined (x,y,z and $U_{\text {iso }}$ ). At convergence, $w R 2=0.1016$ and $\mathrm{Goof}=1.018$ for 444 variables refined against 5561 data. As a comparison for refinement on F, R1 $=0.0364$ for those 4427 data with $\mathrm{I}>2.0 \sigma(\mathrm{I})$.

Definitions:

$$
\mathrm{wR} 2=\left[\Sigma\left[\mathrm{w}\left(\mathrm{F}_{\mathrm{o}}^{2}-\mathrm{F}_{\mathrm{c}}^{2}\right)^{2}\right] / \Sigma\left[\mathrm{w}\left(\mathrm{F}_{\mathrm{o}}^{2}\right)^{2}\right]\right]^{1 / 2}
$$

$\mathrm{R} 1=\Sigma|| \mathrm{F}_{\mathrm{o}}|-| \mathrm{F}_{\mathrm{c}} \| / \Sigma\left|\mathrm{F}_{\mathrm{o}}\right|$

Goof $=\mathrm{S}=\left[\Sigma\left[\mathrm{w}\left(\mathrm{F}_{\mathrm{o}}{ }^{2}-\mathrm{F}_{\mathrm{c}}{ }^{2}\right)^{2}\right] /(\mathrm{n}-\mathrm{p})\right]^{1 / 2}$ where $\mathrm{n}$ is the number of reflections and $\mathrm{p}$ is the total

number of parameters refined.

The thermal ellipsoid plot is shown at the 50\% probability level.

Table 1. Crystal data and structure refinement for S-21.

Identification code

Empirical formula

Formula weight

Temperature

Wavelength

Crystal system

Space group

Unit cell dimensions

Volume

Z
S-21 (Glen Baghdasarian)

$\mathrm{C}_{25} \mathrm{H}_{32} \mathrm{~N}_{4} \mathrm{O}_{6}$

484.55

163(2) K

$0.71073 \AA$

Triclinic

$P \overline{1}$

$\mathrm{a}=8.5415(11) \AA \quad \alpha=113.890(2)^{\circ}$.

$\mathrm{b}=11.5119(15) \AA \quad \beta=94.826(2)^{\circ}$.

$\mathrm{c}=13.4368(18) \AA \quad \gamma=91.961(2)^{\circ}$. 
Density (calculated)

Absorption coefficient

$\mathrm{F}(000)$

Crystal color

Crystal size

Theta range for data collection

Index ranges

Reflections collected

Independent reflections

Completeness to theta $=28.28^{\circ}$

Absorption correction

Max. and min. transmission

Refinement method

Data / restraints / parameters

Goodness-of-fit on $\mathrm{F}^{2}$
$1.341 \mathrm{Mg} / \mathrm{m}^{3}$

$0.097 \mathrm{~mm}^{-1}$

516

yellow

$0.35 \times 0.27 \times 0.19 \mathrm{~mm}^{3}$

1.94 to $28.28^{\circ}$.

$-11 \leq h \leq 11,-15 \leq k \leq 15,-17 \leq l \leq 17$

12427

$5561[\mathrm{R}(\mathrm{int})=0.0213]$

$93.2 \%$

Semi-empirical from equivalents

0.9818 and 0.9669

Full-matrix least-squares on $\mathrm{F}^{2}$

$5561 / 0 / 444$

1.018

Final $\mathrm{R}$ indices $[\mathrm{I}>2 \operatorname{sigma}(\mathrm{I})=4427$ data]

$\mathrm{R}$ indices (all data)

$\mathrm{R} 1=0.0364, \mathrm{wR} 2=0.0922$

$\mathrm{R} 1=0.0502, \mathrm{wR} 2=0.1016$

Largest diff. peak and hole
0.293 and -0.204 e. $\AA^{-3}$

Table 2. Atomic coordinates ( $\mathrm{x} 10^{4}$ ) and equivalent isotropic displacement parameters $\left(\AA^{2} \times 10^{3}\right)$

for $\mathbf{S - 2 1}$. U(eq) is defined as one third of the trace of the orthogonalized $U^{i j}$ tensor.

\begin{tabular}{lcccc}
\hline & & & \\
& & & & \\
& & & & \\
& & & & \\
\hline & & & & \\
$\mathrm{O}(1)$ & $9339(1)$ & $922(1)$ & $4390(1)$ & $23(1)$ \\
$\mathrm{O}(2)$ & $4690(1)$ & $2272(1)$ & $3620(1)$ & $27(1)$ \\
$\mathrm{O}(3)$ & $4367(1)$ & $-7062(1)$ & $-3023(1)$ & $37(1)$ \\
$\mathrm{O}(4)$ & $2856(1)$ & $-5703(1)$ & $-3275(1)$ & $39(1)$ \\
$\mathrm{O}(5)$ & $4847(1)$ & $-701(1)$ & $755(1)$ & $29(1)$ \\
$\mathrm{O}(6)$ & $3131(1)$ & $-1452(1)$ & $-675(1)$ & $32(1)$ \\
$\mathrm{N}(1)$ & $8355(1)$ & $-2342(1)$ & $1560(1)$ & $21(1)$ \\
$\mathrm{N}(2)$ & $7007(1)$ & $-2100(1)$ & $1050(1)$ & $22(1)$ \\
$\mathrm{N}(3)$ & $3910(1)$ & $-5980(1)$ & $-2744(1)$ & $28(1)$
\end{tabular}




$\begin{array}{lrrrr}\mathrm{N}(4) & 4247(1) & -1584(1) & -100(1) & 23(1) \\ \mathrm{C}(1) & 8355(1) & 849(1) & 3439(1) & 19(1) \\ \mathrm{C}(2) & 6760(1) & 279(1) & 3532(1) & 21(1) \\ \mathrm{C}(3) & 6016(2) & 1166(1) & 4543(1) & 24(1) \\ \mathrm{C}(4) & 5835(2) & 2475(1) & 4533(1) & 25(1) \\ \mathrm{C}(5) & 7408(2) & 3057(1) & 4426(1) & 26(1) \\ \mathrm{C}(6) & 8157(2) & 2167(1) & 3427(1) & 23(1) \\ \mathrm{C}(7) & 9064(1) & 3(1) & 2375(1) & 20(1) \\ \mathrm{C}(8) & 9279(1) & -1365(1) & 2194(1) & 20(1) \\ \mathrm{C}(9) & 10758(1) & -1642(1) & 2743(1) & 22(1) \\ \mathrm{C}(10) & 12220(2) & -1369(1) & 2262(1) & 31(1) \\ \mathrm{C}(11) & 10707(2) & -3007(1) & 2657(1) & 26(1) \\ \mathrm{C}(12) & 10840(2) & 1612(1) & 4628(1) & 30(1) \\ \mathrm{C}(13) & 3944(2) & 3370(1) & 3677(1) & 28(1) \\ \mathrm{C}(14) & 2708(1) & 3007(1) & 2714(1) & 24(1) \\ \mathrm{C}(15) & 2657(2) & 1833(1) & 1824(1) & 27(1) \\ \mathrm{C}(16) & 1507(2) & 1514(1) & 941(1) & 32(1) \\ \mathrm{C}(17) & 414(2) & 2373(2) & 931(1) & 34(1) \\ \mathrm{C}(18) & 464(2) & 3548(1) & 1811(1) & 33(1) \\ \mathrm{C}(19) & 1596(2) & 3863(1) & 2697(1) & 29(1) \\ \mathrm{C}(20) & 6231(1) & -3032(1) & 153(1) & 20(1) \\ \mathrm{C}(21) & 6762(1) & -4283(1) & -272(1) & 23(1) \\ \mathrm{C}(22) & 6000(2) & -5234(1) & -1192(1) & 24(1) \\ \mathrm{C}(23) & 4684(1) & -4979(1) & -1743(1) & 23(1) \\ \mathrm{C}(24) & 4126(1) & -3788(1) & -1378(1) & 22(1) \\ \mathrm{C}(25) & 4875(1) & -2826(1) & -432(1) & 21(1)\end{array}$

Table 3. Bond lengths $[\AA]$ and angles $\left[{ }^{\circ}\right]$ for $\mathbf{S - 2 1}$.

\begin{tabular}{ll}
\hline $\mathrm{O}(1)-\mathrm{C}(12)$ & $1.4254(16)$ \\
$\mathrm{O}(1)-\mathrm{C}(1)$ & $1.4398(13)$ \\
$\mathrm{O}(2)-\mathrm{C}(13)$ & $1.4123(15)$ \\
$\mathrm{O}(2)-\mathrm{C}(4)$ & $1.4369(14)$ \\
$\mathrm{O}(3)-\mathrm{N}(3)$ & $1.2341(14)$
\end{tabular}




\begin{tabular}{|c|c|}
\hline $\mathrm{O}(4)-\mathrm{N}(3)$ & $1.2318(15)$ \\
\hline $\mathrm{O}(5)-\mathrm{N}(4)$ & $1.2392(13)$ \\
\hline $\mathrm{O}(6)-\mathrm{N}(4)$ & $1.2290(13)$ \\
\hline $\mathrm{N}(1)-\mathrm{C}(8)$ & $1.2900(15)$ \\
\hline $\mathrm{N}(1)-\mathrm{N}(2)$ & $1.3847(13)$ \\
\hline $\mathrm{N}(2)-\mathrm{C}(20)$ & $1.3457(15)$ \\
\hline $\mathrm{N}(3)-\mathrm{C}(23)$ & $1.4550(15)$ \\
\hline $\mathrm{N}(4)-\mathrm{C}(25)$ & $1.4509(15)$ \\
\hline$C(1)-C(2)$ & $1.5293(16)$ \\
\hline$C(1)-C(6)$ & $1.5389(15)$ \\
\hline$C(1)-C(7)$ & $1.5549(16)$ \\
\hline$C(2)-C(3)$ & $1.5348(16)$ \\
\hline$C(3)-C(4)$ & $1.5250(17)$ \\
\hline$C(4)-C(5)$ & $1.5246(19)$ \\
\hline$C(5)-C(6)$ & $1.5292(17)$ \\
\hline$C(7)-C(8)$ & $1.5132(16)$ \\
\hline$C(8)-C(9)$ & $1.5178(16)$ \\
\hline C(9)-C(11) & $1.5270(17)$ \\
\hline$C(9)-C(10)$ & $1.5327(18)$ \\
\hline$C(13)-C(14)$ & $1.5051(18)$ \\
\hline$C(14)-C(15)$ & $1.3922(17)$ \\
\hline$C(14)-C(19)$ & $1.3972(18)$ \\
\hline$C(15)-C(16)$ & $1.3895(19)$ \\
\hline$C(16)-C(17)$ & $1.386(2)$ \\
\hline $\mathrm{C}(17)-\mathrm{C}(18)$ & $1.387(2)$ \\
\hline C(18)-C(19) & $1.385(2)$ \\
\hline $\mathrm{C}(20)-\mathrm{C}(21)$ & $1.4245(16)$ \\
\hline$C(20)-C(25)$ & $1.4256(16)$ \\
\hline $\mathrm{C}(21)-\mathrm{C}(22)$ & $1.3672(17)$ \\
\hline$C(22)-C(23)$ & $1.3982(18)$ \\
\hline $\mathrm{C}(23)-\mathrm{C}(24)$ & $1.3741(17)$ \\
\hline$C(24)-C(25)$ & $1.3882(16)$ \\
\hline $\mathrm{C}(12)-\mathrm{O}(1)-\mathrm{C}(1)$ & $117.42(9)$ \\
\hline $\mathrm{C}(13)-\mathrm{O}(2)-\mathrm{C}(4)$ & $114.67(9)$ \\
\hline $\mathrm{C}(8)-\mathrm{N}(1)-\mathrm{N}(2)$ & $116.54(10)$ \\
\hline $\mathrm{C}(20)-\mathrm{N}(2)-\mathrm{N}(1)$ & $120.19(10)$ \\
\hline
\end{tabular}




\begin{tabular}{|c|c|}
\hline $\mathrm{O}(4)-\mathrm{N}(3)-\mathrm{O}(3)$ & $123.51(10)$ \\
\hline $\mathrm{O}(4)-\mathrm{N}(3)-\mathrm{C}(23)$ & $118.62(11)$ \\
\hline $\mathrm{O}(3)-\mathrm{N}(3)-\mathrm{C}(23)$ & $117.87(11)$ \\
\hline $\mathrm{O}(6)-\mathrm{N}(4)-\mathrm{O}(5)$ & $122.51(10)$ \\
\hline $\mathrm{O}(6)-\mathrm{N}(4)-\mathrm{C}(25)$ & $118.62(10)$ \\
\hline $\mathrm{O}(5)-\mathrm{N}(4)-\mathrm{C}(25)$ & $118.86(9)$ \\
\hline $\mathrm{O}(1)-\mathrm{C}(1)-\mathrm{C}(2)$ & $104.32(8)$ \\
\hline $\mathrm{O}(1)-\mathrm{C}(1)-\mathrm{C}(6)$ & $112.40(9)$ \\
\hline $\mathrm{C}(2)-\mathrm{C}(1)-\mathrm{C}(6)$ & $109.41(10)$ \\
\hline $\mathrm{O}(1)-\mathrm{C}(1)-\mathrm{C}(7)$ & $110.52(9)$ \\
\hline$C(2)-C(1)-C(7)$ & $112.18(9)$ \\
\hline$C(6)-C(1)-C(7)$ & $108.03(9)$ \\
\hline$C(1)-C(2)-C(3)$ & $111.75(10)$ \\
\hline$C(4)-C(3)-C(2)$ & $111.33(10)$ \\
\hline $\mathrm{O}(2)-\mathrm{C}(4)-\mathrm{C}(5)$ & $111.83(10)$ \\
\hline $\mathrm{O}(2)-\mathrm{C}(4)-\mathrm{C}(3)$ & $105.76(10)$ \\
\hline$C(5)-C(4)-C(3)$ & $110.89(10)$ \\
\hline$C(4)-C(5)-C(6)$ & $111.72(10)$ \\
\hline$C(5)-C(6)-C(1)$ & $111.96(10)$ \\
\hline $\mathrm{C}(8)-\mathrm{C}(7)-\mathrm{C}(1)$ & $116.72(9)$ \\
\hline $\mathrm{N}(1)-\mathrm{C}(8)-\mathrm{C}(7)$ & $125.77(10)$ \\
\hline $\mathrm{N}(1)-\mathrm{C}(8)-\mathrm{C}(9)$ & $116.09(10)$ \\
\hline $\mathrm{C}(7)-\mathrm{C}(8)-\mathrm{C}(9)$ & $118.02(10)$ \\
\hline$C(8)-C(9)-C(11)$ & $112.77(10)$ \\
\hline $\mathrm{C}(8)-\mathrm{C}(9)-\mathrm{C}(10)$ & $110.47(10)$ \\
\hline$C(11)-C(9)-C(10)$ & $109.82(11)$ \\
\hline $\mathrm{O}(2)-\mathrm{C}(13)-\mathrm{C}(14)$ & $109.09(10)$ \\
\hline$C(15)-C(14)-C(19)$ & $118.79(12)$ \\
\hline$C(15)-C(14)-C(13)$ & $121.28(11)$ \\
\hline$C(19)-C(14)-C(13)$ & $119.92(11)$ \\
\hline$C(16)-C(15)-C(14)$ & $120.49(12)$ \\
\hline$C(17)-C(16)-C(15)$ & $120.25(13)$ \\
\hline$C(16)-C(17)-C(18)$ & $119.65(13)$ \\
\hline $\mathrm{C}(19)-\mathrm{C}(18)-\mathrm{C}(17)$ & $120.27(13)$ \\
\hline$C(18)-C(19)-C(14)$ & $120.55(13)$ \\
\hline $\mathrm{N}(2)-\mathrm{C}(20)-\mathrm{C}(21)$ & $120.78(11)$ \\
\hline
\end{tabular}




$\begin{array}{ll}\mathrm{N}(2)-\mathrm{C}(20)-\mathrm{C}(25) & 122.66(11) \\ \mathrm{C}(21)-\mathrm{C}(20)-\mathrm{C}(25) & 116.54(10) \\ \mathrm{C}(22)-\mathrm{C}(21)-\mathrm{C}(20) & 121.40(11) \\ \mathrm{C}(21)-\mathrm{C}(22)-\mathrm{C}(23) & 119.83(11) \\ \mathrm{C}(24)-\mathrm{C}(23)-\mathrm{C}(22) & 121.45(11) \\ \mathrm{C}(24)-\mathrm{C}(23)-\mathrm{N}(3) & 118.81(11) \\ \mathrm{C}(22)-\mathrm{C}(23)-\mathrm{N}(3) & 119.71(11) \\ \mathrm{C}(23)-\mathrm{C}(24)-\mathrm{C}(25) & 118.97(11) \\ \mathrm{C}(24)-\mathrm{C}(25)-\mathrm{C}(20) & 121.79(11) \\ \mathrm{C}(24)-\mathrm{C}(25)-\mathrm{N}(4) & 116.30(10) \\ \mathrm{C}(20)-\mathrm{C}(25)-\mathrm{N}(4) & 121.85(10)\end{array}$

Table 4. Anisotropic displacement parameters $\left(\AA^{2} \times 10^{3}\right)$ for $\mathbf{S - 2 1}$. The anisotropic displacement factor exponent takes the form: $-2 \pi^{2}\left[h^{2} a^{* 2} U^{11}+\ldots+2 h k a^{*} b^{*} U^{12}\right]$

\begin{tabular}{|c|c|c|c|c|c|c|}
\hline & $\mathrm{U}^{11}$ & $\mathrm{U}^{22}$ & $U^{33}$ & $\mathrm{U}^{23}$ & $\mathrm{U}^{13}$ & $\mathrm{U}^{12}$ \\
\hline $\mathrm{O}(1)$ & $24(1)$ & $24(1)$ & $20(1)$ & $11(1)$ & $-4(1)$ & $-1(1)$ \\
\hline $\mathrm{O}(2)$ & $32(1)$ & $20(1)$ & $28(1)$ & $9(1)$ & $-1(1)$ & $7(1)$ \\
\hline $\mathrm{O}(3)$ & $49(1)$ & $21(1)$ & $34(1)$ & $2(1)$ & $6(1)$ & $-4(1)$ \\
\hline $\mathrm{O}(4)$ & $42(1)$ & $35(1)$ & $31(1)$ & $8(1)$ & $-9(1)$ & $-9(1)$ \\
\hline $\mathrm{O}(5)$ & $34(1)$ & 21(1) & $27(1)$ & $4(1)$ & $-3(1)$ & $3(1)$ \\
\hline $\mathrm{O}(6)$ & $29(1)$ & $28(1)$ & $38(1)$ & $13(1)$ & $-9(1)$ & $3(1)$ \\
\hline $\mathrm{N}(1)$ & 21(1) & $22(1)$ & $21(1)$ & $8(1)$ & 1(1) & $3(1)$ \\
\hline $\mathrm{N}(2)$ & $22(1)$ & 19(1) & $22(1)$ & $6(1)$ & $-1(1)$ & $3(1)$ \\
\hline $\mathrm{N}(3)$ & $31(1)$ & $25(1)$ & $23(1)$ & $6(1)$ & $4(1)$ & $-7(1)$ \\
\hline $\mathrm{N}(4)$ & $22(1)$ & 21(1) & $25(1)$ & $9(1)$ & 1(1) & $0(1)$ \\
\hline$C(1)$ & $23(1)$ & $17(1)$ & $18(1)$ & $8(1)$ & $-2(1)$ & $0(1)$ \\
\hline$C(2)$ & $24(1)$ & $16(1)$ & $23(1)$ & $9(1)$ & $1(1)$ & $1(1)$ \\
\hline$C(3)$ & $28(1)$ & $23(1)$ & $24(1)$ & $12(1)$ & $5(1)$ & $4(1)$ \\
\hline$C(4)$ & $33(1)$ & $21(1)$ & $20(1)$ & $7(1)$ & $3(1)$ & $6(1)$ \\
\hline$C(5)$ & $35(1)$ & $16(1)$ & $24(1)$ & $7(1)$ & $-2(1)$ & $2(1)$ \\
\hline$C(6)$ & 29(1) & $18(1)$ & $23(1)$ & $10(1)$ & $0(1)$ & $-1(1)$ \\
\hline
\end{tabular}




$\begin{array}{lllllll}\mathrm{C}(7) & 23(1) & 19(1) & 19(1) & 9(1) & 0(1) & 0(1) \\ \mathrm{C}(8) & 22(1) & 21(1) & 18(1) & 8(1) & 4(1) & 3(1) \\ \mathrm{C}(9) & 24(1) & 21(1) & 22(1) & 9(1) & -1(1) & 2(1) \\ \mathrm{C}(10) & 23(1) & 31(1) & 42(1) & 20(1) & 3(1) & 3(1) \\ \mathrm{C}(11) & 28(1) & 23(1) & 30(1) & 14(1) & 2(1) & 4(1) \\ \mathrm{C}(12) & 28(1) & 29(1) & 28(1) & 10(1) & -5(1) & -4(1) \\ \mathrm{C}(13) & 34(1) & 18(1) & 32(1) & 10(1) & 1(1) & 5(1) \\ \mathrm{C}(14) & 26(1) & 20(1) & 30(1) & 14(1) & 4(1) & 0(1) \\ \mathrm{C}(15) & 30(1) & 23(1) & 30(1) & 13(1) & 7(1) & 1(1) \\ \mathrm{C}(16) & 36(1) & 31(1) & 29(1) & 13(1) & 5(1) & -7(1) \\ \mathrm{C}(17) & 28(1) & 43(1) & 36(1) & 25(1) & -2(1) & -9(1) \\ \mathrm{C}(18) & 27(1) & 35(1) & 48(1) & 27(1) & 3(1) & 1(1) \\ \mathrm{C}(19) & 29(1) & 22(1) & 38(1) & 15(1) & 5(1) & 2(1) \\ \mathrm{C}(20) & 20(1) & 20(1) & 21(1) & 9(1) & 4(1) & -1(1) \\ \mathrm{C}(21) & 22(1) & 21(1) & 25(1) & 8(1) & 4(1) & 2(1) \\ \mathrm{C}(22) & 25(1) & 20(1) & 27(1) & 7(1) & 8(1) & 0(1) \\ \mathrm{C}(23) & 26(1) & 20(1) & 20(1) & 5(1) & 5(1) & -5(1) \\ \mathrm{C}(24) & 21(1) & 24(1) & 23(1) & 10(1) & 2(1) & -3(1) \\ \mathrm{C}(25) & 21(1) & 20(1) & 22(1) & 9(1) & 3(1) & 0(1)\end{array}$

Table 5. Hydrogen coordinates ( $\mathrm{x} 10^{4}$ ) and isotropic displacement parameters $\left(\AA^{2} \times 10^{3}\right)$ for $\mathbf{S - 2 1 .}$

\begin{tabular}{lcccc}
\hline \multicolumn{1}{c}{ x } & y & z & U(eq) \\
\hline & & & & \\
\hline & & & & \\
$\mathrm{H}(2)$ & $6650(19)$ & $-1357(16)$ & $1270(13)$ & $33(4)$ \\
$\mathrm{H}(2 \mathrm{~A})$ & $6886(17)$ & $-539(15)$ & $3573(12)$ & $29(4)$ \\
$\mathrm{H}(2 \mathrm{~B})$ & $6038(15)$ & $125(12)$ & $2870(11)$ & $17(3)$ \\
$\mathrm{H}(3 \mathrm{~A})$ & $4965(19)$ & $820(14)$ & $4570(12)$ & $32(4)$ \\
$\mathrm{H}(3 \mathrm{~B})$ & $6674(17)$ & $1272(13)$ & $5204(12)$ & $24(3)$ \\
$\mathrm{H}(4)$ & $5408(17)$ & $3052(14)$ & $5205(12)$ & $29(4)$
\end{tabular}




\begin{tabular}{|c|c|c|c|c|}
\hline $\mathrm{H}(5 \mathrm{~A})$ & $8135(17)$ & $3231(13)$ & $5101(12)$ & $26(4)$ \\
\hline $\mathrm{H}(5 \mathrm{~B})$ & $7270(17)$ & $3885(15)$ & $4375(12)$ & $29(4)$ \\
\hline $\mathrm{H}(6 \mathrm{~A})$ & $9175(18)$ & $2568(14)$ & $3394(12)$ & $27(4)$ \\
\hline $\mathrm{H}(6 \mathrm{~B})$ & $7466(17)$ & $2030(13)$ & $2753(12)$ & $24(3)$ \\
\hline $\mathrm{H}(7 \mathrm{~A})$ & $10097(18)$ & $403(14)$ & $2384(11)$ & $26(4)$ \\
\hline $\mathrm{H}(7 \mathrm{~B})$ & $8399(16)$ & $41(12)$ & $1757(11)$ & $19(3)$ \\
\hline $\mathrm{H}(9)$ & $10852(16)$ & $-1069(13)$ & $3525(12)$ & $23(3)$ \\
\hline $\mathrm{H}(10 \mathrm{~A})$ & $12120(19)$ & $-1932(16)$ & $1455(14)$ & $37(4)$ \\
\hline $\mathrm{H}(10 \mathrm{~B})$ & 13176(19) & $-1520(15)$ & $2644(12)$ & $33(4)$ \\
\hline $\mathrm{H}(10 \mathrm{C})$ & $12330(20)$ & $-525(18)$ & 2311(14) & $44(5)$ \\
\hline $\mathrm{H}(11 \mathrm{~A})$ & 11651(18) & $-3136(14)$ & $3054(12)$ & $28(4)$ \\
\hline $\mathrm{H}(11 \mathrm{~B})$ & 9796(19) & $-3210(15)$ & $2970(13)$ & $35(4)$ \\
\hline $\mathrm{H}(11 \mathrm{C})$ & $10635(18)$ & $-3651(15)$ & $1890(13)$ & $31(4)$ \\
\hline $\mathrm{H}(12 \mathrm{~A})$ & $11460(20)$ & $1345(16)$ & $3999(14)$ & $39(4)$ \\
\hline $\mathrm{H}(12 \mathrm{~B})$ & $10750(20)$ & $2534(17)$ & 4889(13) & $42(4)$ \\
\hline $\mathrm{H}(12 \mathrm{C})$ & $11420(20)$ & $1430(16)$ & $5199(14)$ & $41(4)$ \\
\hline $\mathrm{H}(13 \mathrm{~A})$ & $4750(20)$ & $4036(18)$ & $3684(14)$ & $48(5)$ \\
\hline $\mathrm{H}(13 \mathrm{~B})$ & $3453(19)$ & $3723(15)$ & $4356(13)$ & $35(4)$ \\
\hline $\mathrm{H}(15)$ & $3447(19)$ & $1241(15)$ & $1812(12)$ & $35(4)$ \\
\hline $\mathrm{H}(16)$ & $1490(20)$ & $703(17)$ & $324(14)$ & $42(4)$ \\
\hline $\mathrm{H}(17)$ & $-380(20)$ & $2139(16)$ & $322(14)$ & $42(4)$ \\
\hline $\mathrm{H}(18)$ & $-300(20)$ & $4154(16)$ & $1820(13)$ & $39(4)$ \\
\hline $\mathrm{H}(19)$ & $1619(18)$ & $4692(16)$ & $3329(13)$ & $36(4)$ \\
\hline $\mathrm{H}(21)$ & $7670(17)$ & $-4443(13)$ & $113(11)$ & $23(3)$ \\
\hline $\mathrm{H}(22)$ & $6387(17)$ & $-6065(15)$ & $-1491(12)$ & $29(4)$ \\
\hline $\mathrm{H}(24)$ & $3261(19)$ & $-3622(14)$ & $-1747(12)$ & $32(4)$ \\
\hline
\end{tabular}

Table 6. Torsion angles $\left[{ }^{\circ}\right]$ for $\mathbf{S - 2 1}$.

\begin{tabular}{lc}
\hline $\mathrm{C}(8)-\mathrm{N}(1)-\mathrm{N}(2)-\mathrm{C}(20)$ & $160.41(11)$ \\
$\mathrm{C}(12)-\mathrm{O}(1)-\mathrm{C}(1)-\mathrm{C}(2)$ & $-169.95(10)$ \\
$\mathrm{C}(12)-\mathrm{O}(1)-\mathrm{C}(1)-\mathrm{C}(6)$ & $-51.50(14)$ \\
$\mathrm{C}(12)-\mathrm{O}(1)-\mathrm{C}(1)-\mathrm{C}(7)$ & $69.28(12)$ \\
$\mathrm{O}(1)-\mathrm{C}(1)-\mathrm{C}(2)-\mathrm{C}(3)$ & $64.41(11)$
\end{tabular}




\begin{tabular}{|c|c|}
\hline$C(6)-C(1)-C(2)-C(3)$ & $-56.06(12)$ \\
\hline$C(7)-C(1)-C(2)-C(3)$ & $-175.94(9)$ \\
\hline$C(1)-C(2)-C(3)-C(4)$ & $56.73(13)$ \\
\hline$C(13)-O(2)-C(4)-C(5)$ & $-80.25(13)$ \\
\hline $\mathrm{C}(13)-\mathrm{O}(2)-\mathrm{C}(4)-\mathrm{C}(3)$ & $158.94(10)$ \\
\hline $\mathrm{C}(2)-\mathrm{C}(3)-\mathrm{C}(4)-\mathrm{O}(2)$ & $66.45(13)$ \\
\hline$C(2)-C(3)-C(4)-C(5)$ & $-54.96(13)$ \\
\hline $\mathrm{O}(2)-\mathrm{C}(4)-\mathrm{C}(5)-\mathrm{C}(6)$ & $-63.24(13)$ \\
\hline$C(3)-C(4)-C(5)-C(6)$ & $54.54(13)$ \\
\hline$C(4)-C(5)-C(6)-C(1)$ & $-55.54(13)$ \\
\hline $\mathrm{O}(1)-\mathrm{C}(1)-\mathrm{C}(6)-\mathrm{C}(5)$ & $-59.88(13)$ \\
\hline$C(2)-C(1)-C(6)-C(5)$ & $55.52(12)$ \\
\hline$C(7)-C(1)-C(6)-C(5)$ & $177.91(10)$ \\
\hline $\mathrm{O}(1)-\mathrm{C}(1)-\mathrm{C}(7)-\mathrm{C}(8)$ & $59.66(13)$ \\
\hline$C(2)-C(1)-C(7)-C(8)$ & $-56.31(13)$ \\
\hline$C(6)-C(1)-C(7)-C(8)$ & $-176.99(10)$ \\
\hline $\mathrm{N}(2)-\mathrm{N}(1)-\mathrm{C}(8)-\mathrm{C}(7)$ & $-3.72(16)$ \\
\hline $\mathrm{N}(2)-\mathrm{N}(1)-\mathrm{C}(8)-\mathrm{C}(9)$ & $-179.64(9)$ \\
\hline $\mathrm{C}(1)-\mathrm{C}(7)-\mathrm{C}(8)-\mathrm{N}(1)$ & $100.60(13)$ \\
\hline$C(1)-C(7)-C(8)-C(9)$ & $-83.55(13)$ \\
\hline $\mathrm{N}(1)-\mathrm{C}(8)-\mathrm{C}(9)-\mathrm{C}(11)$ & $-13.38(15)$ \\
\hline$C(7)-C(8)-C(9)-C(11)$ & $170.37(10)$ \\
\hline $\mathrm{N}(1)-\mathrm{C}(8)-\mathrm{C}(9)-\mathrm{C}(10)$ & $109.93(12)$ \\
\hline$C(7)-C(8)-C(9)-C(10)$ & $-66.32(13)$ \\
\hline $\mathrm{C}(4)-\mathrm{O}(2)-\mathrm{C}(13)-\mathrm{C}(14)$ & $-176.34(10)$ \\
\hline $\mathrm{O}(2)-\mathrm{C}(13)-\mathrm{C}(14)-\mathrm{C}(15)$ & $-13.66(16)$ \\
\hline $\mathrm{O}(2)-\mathrm{C}(13)-\mathrm{C}(14)-\mathrm{C}(19)$ & $167.18(11)$ \\
\hline$C(19)-C(14)-C(15)-C(16)$ & $-0.78(18)$ \\
\hline$C(13)-C(14)-C(15)-C(16)$ & $-179.95(12)$ \\
\hline$C(14)-C(15)-C(16)-C(17)$ & $1.07(19)$ \\
\hline$C(15)-C(16)-C(17)-C(18)$ & $-0.60(19)$ \\
\hline$C(16)-C(17)-C(18)-C(19)$ & $-0.1(2)$ \\
\hline$C(17)-C(18)-C(19)-C(14)$ & $0.4(2)$ \\
\hline$C(15)-C(14)-C(19)-C(18)$ & $0.05(18)$ \\
\hline$C(13)-C(14)-C(19)-C(18)$ & $179.23(12)$ \\
\hline $\mathrm{N}(1)-\mathrm{N}(2)-\mathrm{C}(20)-\mathrm{C}(21)$ & $1.29(16)$ \\
\hline
\end{tabular}




\begin{tabular}{lc}
$\mathrm{N}(1)-\mathrm{N}(2)-\mathrm{C}(20)-\mathrm{C}(25)$ & $-177.38(10)$ \\
$\mathrm{N}(2)-\mathrm{C}(20)-\mathrm{C}(21)-\mathrm{C}(22)$ & $-178.98(11)$ \\
$\mathrm{C}(25)-\mathrm{C}(20)-\mathrm{C}(21)-\mathrm{C}(22)$ & $-0.23(17)$ \\
$\mathrm{C}(20)-\mathrm{C}(21)-\mathrm{C}(22)-\mathrm{C}(23)$ & $0.90(18)$ \\
$\mathrm{C}(21)-\mathrm{C}(22)-\mathrm{C}(23)-\mathrm{C}(24)$ & $-0.27(18)$ \\
$\mathrm{C}(21)-\mathrm{C}(22)-\mathrm{C}(23)-\mathrm{N}(3)$ & $177.75(11)$ \\
$\mathrm{O}(4)-\mathrm{N}(3)-\mathrm{C}(23)-\mathrm{C}(24)$ & $5.30(16)$ \\
$\mathrm{O}(3)-\mathrm{N}(3)-\mathrm{C}(23)-\mathrm{C}(24)$ & $-175.70(11)$ \\
$\mathrm{O}(4)-\mathrm{N}(3)-\mathrm{C}(23)-\mathrm{C}(22)$ & $-172.77(11)$ \\
$\mathrm{O}(3)-\mathrm{N}(3)-\mathrm{C}(23)-\mathrm{C}(22)$ & $6.22(16)$ \\
$\mathrm{C}(22)-\mathrm{C}(23)-\mathrm{C}(24)-\mathrm{C}(25)$ & $-1.02(18)$ \\
$\mathrm{N}(3)-\mathrm{C}(23)-\mathrm{C}(24)-\mathrm{C}(25)$ & $-179.06(10)$ \\
$\mathrm{C}(23)-\mathrm{C}(24)-\mathrm{C}(25)-\mathrm{C}(20)$ & $1.71(17)$ \\
$\mathrm{C}(23)-\mathrm{C}(24)-\mathrm{C}(25)-\mathrm{N}(4)$ & $178.97(10)$ \\
$\mathrm{N}(2)-\mathrm{C}(20)-\mathrm{C}(25)-\mathrm{C}(24)$ & $177.63(11)$ \\
$\mathrm{C}(21)-\mathrm{C}(20)-\mathrm{C}(25)-\mathrm{C}(24)$ & $-1.09(16)$ \\
$\mathrm{N}(2)-\mathrm{C}(20)-\mathrm{C}(25)-\mathrm{N}(4)$ & $0.53(17)$ \\
$\mathrm{C}(21)-\mathrm{C}(20)-\mathrm{C}(25)-\mathrm{N}(4)$ & $-178.19(10)$ \\
$\mathrm{O}(6)-\mathrm{N}(4)-\mathrm{C}(25)-\mathrm{C}(24)$ & $-1.44(16)$ \\
$\mathrm{O}(5)-\mathrm{N}(4)-\mathrm{C}(25)-\mathrm{C}(24)$ & $178.08(10)$ \\
$\mathrm{O}(6)-\mathrm{N}(4)-\mathrm{C}(25)-\mathrm{C}(20)$ & $175.81(10)$ \\
$\mathrm{O}(5)-\mathrm{N}(4)-\mathrm{C}(25)-\mathrm{C}(20)$ & $-4.66(16)$ \\
\hline &
\end{tabular}

\section{Carbamate $\mathrm{S}-23$}

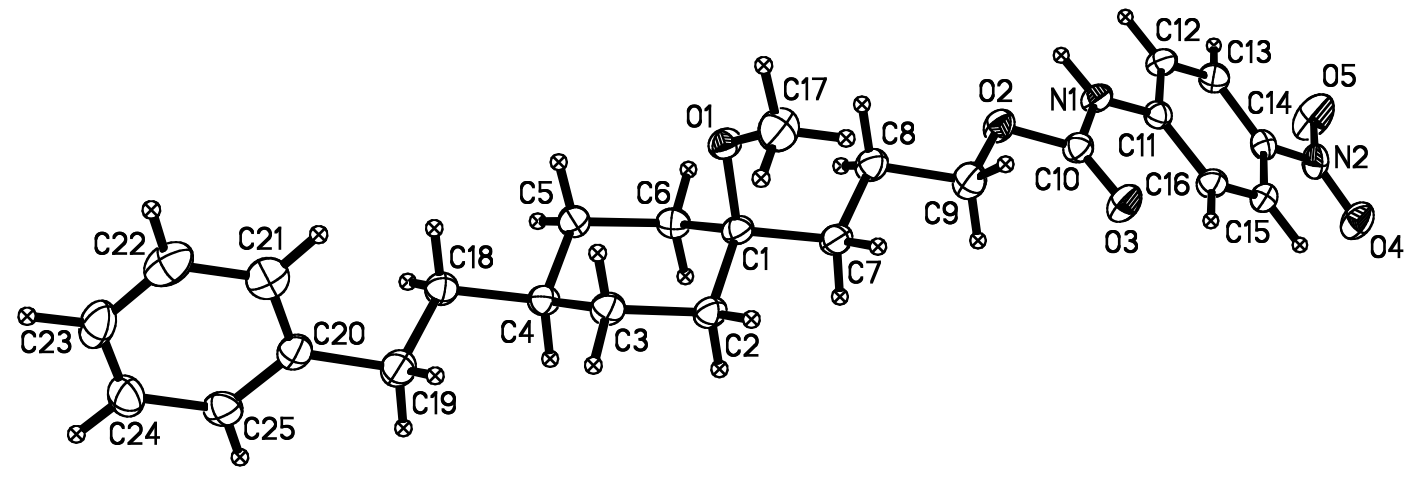

X-ray Data Collection, Structure Solution and Refinement for S-23. 
A colorless crystal of approximate dimensions $0.09 \times 0.28 \times 0.42 \mathrm{~mm}$ was mounted on a glass fiber and transferred to a Bruker CCD platform diffractometer. The SMART ${ }^{1}$ program package was used to determine the unit-cell parameters and for data collection (25 sec/frame scan time for a sphere of diffraction data). The raw frame data was processed using $\mathrm{SAINT}^{2}$ and $\mathrm{SADABS}^{3}$ to yield the reflection data file. Subsequent calculations were carried out using the SHELXTL ${ }^{4}$ program. There were no systematic absences nor any diffraction symmetry other than the Friedel condition. The centrosymmetric triclinic space group $\mathrm{P} \overline{1}$ was assigned and later determined to be correct.

The structure was solved by direct methods and refined on $\mathrm{F}^{2}$ by full-matrix least-squares techniques. The analytical scattering factors ${ }^{5}$ for neutral atoms were used throughout the analysis. Hydrogen atoms were located from a difference-Fourier map and refined (x,y,z and $\left.\mathrm{U}_{\text {iso }}\right)$. At convergence, $w R 2=0.1046$ and $\mathrm{Goof}=1.010$ for 417 variables refined against 4592 data. As a comparison for refinement on $\mathrm{F}, \mathrm{R} 1=0.0383$ for those 3434 data with $\mathrm{I}>2.0 \sigma(\mathrm{I})$.

Definitions:

$$
\begin{aligned}
& \mathrm{wR} 2=\left[\Sigma\left[\mathrm{w}\left(\mathrm{F}_{\mathrm{o}}^{2}-\mathrm{F}_{\mathrm{c}}{ }^{2}\right)^{2}\right] / \Sigma\left[\mathrm{w}\left(\mathrm{F}_{\mathrm{o}}{ }^{2}\right)^{2}\right]\right]^{1 / 2} \\
& \mathrm{R} 1=\Sigma|| \mathrm{F}_{\mathrm{o}}|-| \mathrm{F}_{\mathrm{c}} \| / \Sigma\left|\mathrm{F}_{\mathrm{o}}\right|
\end{aligned}
$$

Goof $=\mathrm{S}=\left[\Sigma\left[\mathrm{w}\left(\mathrm{F}_{\mathrm{o}}{ }^{2}-\mathrm{F}_{\mathrm{c}}{ }^{2}\right)^{2}\right] /(\mathrm{n}-\mathrm{p})\right]^{1 / 2}$ where $\mathrm{n}$ is the number of reflections and $\mathrm{p}$ is the total

number of parameters refined.

The thermal ellipsoid plot is shown at the $50 \%$ probability level.

Table 1. Crystal data and structure refinement for $\mathbf{S - 2 3}$.

Identification code

Empirical formula

Formula weight

Temperature

Wavelength

Crystal system

Space group

Unit cell dimensions

Volume

Z

Density (calculated)
S-23 (Glen Baghdasarian)

$\mathrm{C}_{25} \mathrm{H}_{32} \mathrm{~N}_{2} \mathrm{O}_{5}$

440.53

163(2) K

$0.71073 \AA$

Triclinic

$P \overline{1}$

$\mathrm{a}=8.0052(15) \AA \quad \alpha=87.037(4)^{\circ}$.

$\mathrm{b}=11.777(2) \AA \quad \beta=84.042(3)^{\circ}$.

$\mathrm{c}=12.237(2) \AA \quad \gamma=86.669(3)^{\circ}$.

1144.3(4) $\AA^{3}$

2

$1.279 \mathrm{Mg} / \mathrm{m}^{3}$ 
Absorption coefficient

$\mathrm{F}(000)$

Crystal color

Crystal size

Theta range for data collection

Index ranges

Reflections collected

Independent reflections

Completeness to theta $=26.37^{\circ}$

Absorption correction

Max. and min. transmission

Refinement method

Data / restraints / parameters

Goodness-of-fit on $\mathrm{F}^{2}$

Final $\mathrm{R}$ indices $[\mathrm{I}>2 \operatorname{sigma}(\mathrm{I})=3434$ data]

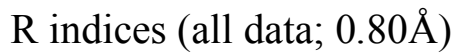

Largest diff. peak and hole
$0.089 \mathrm{~mm}^{-1}$

472

colorless

$0.42 \times 0.28 \times 0.09 \mathrm{~mm}^{3}$

1.67 to $26.37^{\circ}$.

$-10 \leq h \leq 10,-14 \leq k \leq 14,-15 \leq l \leq 15$

10976

$4592[\mathrm{R}(\mathrm{int})=0.0251]$

$98.3 \%$

Semi-empirical from equivalents

0.9920 and 0.9636

Full-matrix least-squares on $\mathrm{F}^{2}$

4592 / 0 / 417

1.010

$\mathrm{R} 1=0.0383, \mathrm{wR} 2=0.0917$

$\mathrm{R} 1=0.0593, \mathrm{wR} 2=0.1046$

0.312 and -0.275 e. $\AA^{-3}$

Table 2. Atomic coordinates ( $\mathrm{x} 10^{4}$ ) and equivalent isotropic displacement parameters $\left(\AA^{2} \times 10^{3}\right)$

for $\mathbf{S - 2 3}$. U(eq) is defined as one third of the trace of the orthogonalized $U^{i j}$ tensor.

\begin{tabular}{|c|c|c|c|c|}
\hline & $\mathrm{x}$ & $\mathrm{y}$ & $\mathrm{z}$ & $\mathrm{U}(\mathrm{eq})$ \\
\hline $\mathrm{O}(1)$ & 1011(1) & 2199(1) & $2034(1)$ & $23(1)$ \\
\hline $\mathrm{O}(2)$ & $-2523(1)$ & $461(1)$ & $-891(1)$ & $26(1)$ \\
\hline $\mathrm{O}(3)$ & $-5192(1)$ & $999(1)$ & $-1211(1)$ & $31(1)$ \\
\hline $\mathrm{O}(4)$ & $-8605(2)$ & $-595(1)$ & $-5802(1)$ & $37(1)$ \\
\hline $\mathrm{O}(5)$ & $-6959(2)$ & $-2054(1)$ & $-6190(1)$ & $52(1)$ \\
\hline $\mathrm{N}(1)$ & $-3751(2)$ & $-514(1)$ & $-2063(1)$ & $24(1)$ \\
\hline $\mathrm{N}(2)$ & $-7424(2)$ & $-1260(1)$ & $-5613(1)$ & $28(1)$ \\
\hline$C(1)$ & $640(2)$ & $3061(1)$ & $1182(1)$ & $21(1)$ \\
\hline $\mathrm{C}(2)$ & $367(2)$ & $4238(1)$ & $1671(1)$ & $23(1)$ \\
\hline $\mathrm{C}(3)$ & $1898(2)$ & $4592(1)$ & 2191(1) & $24(1)$ \\
\hline
\end{tabular}




$\begin{array}{lrrrr}\mathrm{C}(4) & 3491(2) & 4577(1) & 1380(1) & 22(1) \\ \mathrm{C}(5) & 3766(2) & 3420(1) & 851(1) & 22(1) \\ \mathrm{C}(6) & 2221(2) & 3035(1) & 367(1) & 22(1) \\ \mathrm{C}(7) & -919(2) & 2769(1) & 647(1) & 25(1) \\ \mathrm{C}(8) & -857(2) & 1608(1) & 128(1) & 26(1) \\ \mathrm{C}(9) & -2590(2) & 1428(1) & -194(2) & 30(1) \\ \mathrm{C}(10) & -3947(2) & 378(1) & -1373(1) & 23(1) \\ \mathrm{C}(11) & -4755(2) & -702(1) & -2898(1) & 21(1) \\ \mathrm{C}(12) & -4283(2) & -1620(1) & -3568(1) & 25(1) \\ \mathrm{C}(13) & -5152(2) & -1816(1) & -4449(1) & 25(1) \\ \mathrm{C}(14) & -6512(2) & -1081(1) & -4664(1) & 22(1) \\ \mathrm{C}(15) & -7020(2) & -178(1) & -4010(1) & 24(1) \\ \mathrm{C}(16) & -6158(2) & 10(1) & -3122(1) & 24(1) \\ \mathrm{C}(17) & -245(2) & 2058(2) & 2934(2) & 37(1) \\ \mathrm{C}(18) & 5055(2) & 4839(1) & 1926(1) & 25(1) \\ \mathrm{C}(19) & 4958(2) & 5990(1) & 2455(2) & 32(1) \\ \mathrm{C}(20) & 6475(2) & 6189(1) & 3047(1) & 26(1) \\ \mathrm{C}(21) & 6808(2) & 5529(2) & 3985(1) & 32(1) \\ \mathrm{C}(22) & 8164(2) & 5736(2) & 4551(2) & 39(1) \\ \mathrm{C}(23) & 9215(2) & 6596(2) & 4184(2) & 39(1) \\ \mathrm{C}(24) & 8916(2) & 7246(2) & 3252(2) & 35(1) \\ \mathrm{C}(25) & 7555(2) & 7044(1) & 2690(1) & 29(1)\end{array}$

Table 3. Bond lengths $[\AA]$ and angles $\left[{ }^{\circ}\right]$ for $\mathbf{S - 2 3}$.

\begin{tabular}{ll}
\hline $\mathrm{O}(1)-\mathrm{C}(17)$ & $1.422(2)$ \\
$\mathrm{O}(1)-\mathrm{C}(1)$ & $1.4574(17)$ \\
$\mathrm{O}(2)-\mathrm{C}(10)$ & $1.3478(18)$ \\
$\mathrm{O}(2)-\mathrm{C}(9)$ & $1.4522(19)$ \\
$\mathrm{O}(3)-\mathrm{C}(10)$ & $1.2064(18)$ \\
$\mathrm{O}(4)-\mathrm{N}(2)$ & $1.2255(17)$ \\
$\mathrm{O}(5)-\mathrm{N}(2)$ & $1.2189(18)$ \\
$\mathrm{N}(1)-\mathrm{C}(10)$ & $1.3732(19)$ \\
$\mathrm{N}(1)-\mathrm{C}(11)$ & $1.3989(19)$
\end{tabular}




$\begin{array}{lr}\mathrm{N}(2)-\mathrm{C}(14) & 1.4648(19) \\ \mathrm{C}(1)-\mathrm{C}(6) & 1.528(2) \\ \mathrm{C}(1)-\mathrm{C}(7) & 1.532(2) \\ \mathrm{C}(1)-\mathrm{C}(2) & 1.534(2) \\ \mathrm{C}(2)-\mathrm{C}(3) & 1.526(2) \\ \mathrm{C}(3)-\mathrm{C}(4) & 1.532(2) \\ \mathrm{C}(4)-\mathrm{C}(18) & 1.532(2) \\ \mathrm{C}(4)-\mathrm{C}(5) & 1.534(2) \\ \mathrm{C}(5)-\mathrm{C}(6) & 1.526(2) \\ \mathrm{C}(7)-\mathrm{C}(8) & 1.532(2) \\ \mathrm{C}(8)-\mathrm{C}(9) & 1.511(2) \\ \mathrm{C}(11)-\mathrm{C}(12) & 1.401(2) \\ \mathrm{C}(11)-\mathrm{C}(16) & 1.403(2) \\ \mathrm{C}(12)-\mathrm{C}(13) & 1.378(2) \\ \mathrm{C}(13)-\mathrm{C}(14) & 1.389(2) \\ \mathrm{C}(14)-\mathrm{C}(15) & 1.381(2) \\ \mathrm{C}(15)-\mathrm{C}(16) & 1.379(2) \\ \mathrm{C}(18)-\mathrm{C}(19) & 1.528(2) \\ \mathrm{C}(19)-\mathrm{C}(20) & 1.512(2) \\ \mathrm{C}(20)-\mathrm{C}(25) & 1.389(2) \\ \mathrm{C}(20)-\mathrm{C}(21) & 1.393(2) \\ \mathrm{C}(21)-\mathrm{C}(22) & 1.387(3) \\ \mathrm{C}(22)-\mathrm{C}(23) & 1.381(3) \\ \mathrm{C}(23)-\mathrm{C}(24) & 1.374(3) \\ \mathrm{C}(24)-\mathrm{C}(25) & 1.386(2) \\ \mathrm{C}(17)-\mathrm{O}(1)-\mathrm{C}(1) & 116.75(12) \\ \mathrm{C}(10)-\mathrm{O}(2)-\mathrm{C}(9) & 113.01(11) \\ \mathrm{C}(10)-\mathrm{N}(1)-\mathrm{C}(11) & 126.21(13) \\ \mathrm{O}(5)-\mathrm{N}(2)-\mathrm{O}(4) & 122.84(14) \\ \mathrm{O}(5)-\mathrm{N}(2)-\mathrm{C}(14) & 118.54(13) \\ \mathrm{O}(4)-\mathrm{N}(2)-\mathrm{C}(14) & 118.61(13) \\ \mathrm{O}(1)-\mathrm{C}(1)-\mathrm{C}(6) & 104.32(11) \\ \mathrm{O}(1)-\mathrm{C}(1)-\mathrm{C}(7) & 110.36(12) \\ \mathrm{C}(6)-\mathrm{C}(1)-\mathrm{C}(7) & 112.05(13) \\ \mathrm{O}(1)-\mathrm{C}(1)-\mathrm{C}(2) & 110.24(12) \\ \mathrm{C}(6)-\mathrm{C}(1)-\mathrm{C}(2) & 109.50(12) \\ & \end{array}$




$\begin{array}{ll}\mathrm{C}(7)-\mathrm{C}(1)-\mathrm{C}(2) & 110.23(12) \\ \mathrm{C}(3)-\mathrm{C}(2)-\mathrm{C}(1) & 112.75(12) \\ \mathrm{C}(2)-\mathrm{C}(3)-\mathrm{C}(4) & 112.34(13) \\ \mathrm{C}(18)-\mathrm{C}(4)-\mathrm{C}(3) & 112.37(13) \\ \mathrm{C}(18)-\mathrm{C}(4)-\mathrm{C}(5) & 109.86(12) \\ \mathrm{C}(3)-\mathrm{C}(4)-\mathrm{C}(5) & 110.13(12) \\ \mathrm{C}(6)-\mathrm{C}(5)-\mathrm{C}(4) & 113.85(12) \\ \mathrm{C}(5)-\mathrm{C}(6)-\mathrm{C}(1) & 113.23(12) \\ \mathrm{C}(1)-\mathrm{C}(7)-\mathrm{C}(8) & 116.76(12) \\ \mathrm{C}(9)-\mathrm{C}(8)-\mathrm{C}(7) & 107.75(13) \\ \mathrm{O}(2)-\mathrm{C}(9)-\mathrm{C}(8) & 109.93(13) \\ \mathrm{O}(3)-\mathrm{C}(10)-\mathrm{O}(2) & 124.43(14) \\ \mathrm{O}(3)-\mathrm{C}(10)-\mathrm{N}(1) & 126.11(14) \\ \mathrm{O}(2)-\mathrm{C}(10)-\mathrm{N}(1) & 109.46(12) \\ \mathrm{N}(1)-\mathrm{C}(11)-\mathrm{C}(12) & 117.79(13) \\ \mathrm{N}(1)-\mathrm{C}(11)-\mathrm{C}(16) & 123.18(14) \\ \mathrm{C}(12)-\mathrm{C}(11)-\mathrm{C}(16) & 118.98(14) \\ \mathrm{C}(13)-\mathrm{C}(12)-\mathrm{C}(11) & 121.05(14) \\ \mathrm{C}(12)-\mathrm{C}(13)-\mathrm{C}(14) & 118.59(14) \\ \mathrm{C}(15)-\mathrm{C}(14)-\mathrm{C}(13) & 121.64(14) \\ \mathrm{C}(15)-\mathrm{C}(14)-\mathrm{N}(2) & 119.02(13) \\ \mathrm{C}(13)-\mathrm{C}(14)-\mathrm{N}(2) & 119.34(14) \\ \mathrm{C}(16)-\mathrm{C}(15)-\mathrm{C}(14) & 119.65(14) \\ \mathrm{C}(15)-\mathrm{C}(16)-\mathrm{C}(11) & 120.06(14) \\ \mathrm{C}(19)-\mathrm{C}(18)-\mathrm{C}(4) & 114.93(13) \\ \mathrm{C}(20)-\mathrm{C}(19)-\mathrm{C}(18) & 113.50(13) \\ \mathrm{C}(25)-\mathrm{C}(20)-\mathrm{C}(21) & 118.01(15) \\ \mathrm{C}(25)-\mathrm{C}(20)-\mathrm{C}(19) & 121.41(15) \\ \mathrm{C}(21)-\mathrm{C}(20)-\mathrm{C}(19) & 120.57(15) \\ \mathrm{C}(22)-\mathrm{C}(21)-\mathrm{C}(20) & 120.66(17) \\ \mathrm{C}(23)-\mathrm{C}(22)-\mathrm{C}(21) & 120.27(17) \\ \mathrm{C}(24)-\mathrm{C}(23)-\mathrm{C}(22) & 119.82(17) \\ \mathrm{C}(23)-\mathrm{C}(24)-\mathrm{C}(25) & 119.96(17) \\ \mathrm{C}(24)-\mathrm{C}(25)-\mathrm{C}(20) & 121.28(16)\end{array}$


Table 4. Anisotropic displacement parameters $\left(\AA^{2} \times 10^{3}\right)$ for $\mathbf{S - 2 3}$. The anisotropic displacement factor exponent takes the form: $-2 \pi^{2}\left[h^{2} a^{* 2} U^{11}+\ldots+2 h k a^{*} b^{*} U^{12}\right]$

\begin{tabular}{|c|c|c|c|c|c|c|}
\hline & $\mathrm{U}^{11}$ & $\mathrm{U}^{22}$ & $\mathrm{U}^{33}$ & $\mathrm{U}^{23}$ & $\mathrm{U}^{13}$ & $\mathrm{U}^{12}$ \\
\hline $\mathrm{O}(1)$ & $22(1)$ & $23(1)$ & $22(1)$ & $3(1)$ & $-2(1)$ & $1(1)$ \\
\hline $\mathrm{O}(2)$ & $24(1)$ & $26(1)$ & $30(1)$ & $-9(1)$ & $-9(1)$ & $3(1)$ \\
\hline $\mathrm{O}(3)$ & $26(1)$ & $35(1)$ & $34(1)$ & $-12(1)$ & $-9(1)$ & $7(1)$ \\
\hline $\mathrm{O}(4)$ & $30(1)$ & $44(1)$ & $38(1)$ & $-8(1)$ & $-15(1)$ & $4(1)$ \\
\hline $\mathrm{O}(5)$ & $46(1)$ & $60(1)$ & $56(1)$ & $-38(1)$ & $-24(1)$ & $16(1)$ \\
\hline $\mathrm{N}(1)$ & $24(1)$ & $22(1)$ & $25(1)$ & $-4(1)$ & $-8(1)$ & $4(1)$ \\
\hline $\mathrm{N}(2)$ & $22(1)$ & $33(1)$ & $29(1)$ & $-8(1)$ & $-4(1)$ & $-5(1)$ \\
\hline$C(1)$ & $23(1)$ & $20(1)$ & $20(1)$ & $-1(1)$ & $-5(1)$ & $2(1)$ \\
\hline$C(2)$ & $22(1)$ & $22(1)$ & $27(1)$ & $-4(1)$ & $-3(1)$ & $3(1)$ \\
\hline$C(3)$ & $24(1)$ & $22(1)$ & $25(1)$ & $-8(1)$ & $-3(1)$ & $3(1)$ \\
\hline$C(4)$ & $23(1)$ & $20(1)$ & $24(1)$ & $-2(1)$ & $-4(1)$ & $0(1)$ \\
\hline$C(5)$ & $21(1)$ & $22(1)$ & $23(1)$ & $-4(1)$ & $1(1)$ & $0(1)$ \\
\hline$C(6)$ & $27(1)$ & $20(1)$ & $19(1)$ & $-4(1)$ & $-1(1)$ & $0(1)$ \\
\hline$C(7)$ & $24(1)$ & $22(1)$ & $29(1)$ & $-3(1)$ & $-8(1)$ & $3(1)$ \\
\hline$C(8)$ & $26(1)$ & $25(1)$ & $29(1)$ & $-5(1)$ & $-8(1)$ & $2(1)$ \\
\hline$C(9)$ & $29(1)$ & $29(1)$ & $34(1)$ & $-13(1)$ & $-9(1)$ & $1(1)$ \\
\hline$C(10)$ & $23(1)$ & $24(1)$ & $23(1)$ & $-1(1)$ & $-5(1)$ & $0(1)$ \\
\hline $\mathrm{C}(11)$ & $22(1)$ & $21(1)$ & $21(1)$ & $1(1)$ & $-3(1)$ & $-2(1)$ \\
\hline$C(12)$ & $25(1)$ & $22(1)$ & $27(1)$ & $-2(1)$ & $-4(1)$ & $3(1)$ \\
\hline$C(13)$ & $27(1)$ & $23(1)$ & $26(1)$ & $-6(1)$ & $-2(1)$ & $0(1)$ \\
\hline$C(14)$ & $21(1)$ & $24(1)$ & $21(1)$ & $-2(1)$ & $-3(1)$ & $-5(1)$ \\
\hline$C(15)$ & $20(1)$ & $24(1)$ & $28(1)$ & $-1(1)$ & $-5(1)$ & $0(1)$ \\
\hline$C(16)$ & $25(1)$ & $21(1)$ & $27(1)$ & $-6(1)$ & $-4(1)$ & $1(1)$ \\
\hline$C(17)$ & $30(1)$ & $48(1)$ & $31(1)$ & $11(1)$ & $2(1)$ & $-3(1)$ \\
\hline$C(18)$ & $23(1)$ & $23(1)$ & $28(1)$ & $-5(1)$ & $-4(1)$ & $1(1)$ \\
\hline C(19) & $30(1)$ & $28(1)$ & $42(1)$ & $-11(1)$ & $-12(1)$ & $3(1)$ \\
\hline$C(20)$ & $25(1)$ & $25(1)$ & $27(1)$ & $-11(1)$ & $-5(1)$ & $5(1)$ \\
\hline $\mathrm{C}(21)$ & $32(1)$ & $35(1)$ & $29(1)$ & $-5(1)$ & $2(1)$ & $2(1)$ \\
\hline $\mathrm{C}(22)$ & $46(1)$ & $45(1)$ & $26(1)$ & $-6(1)$ & $-10(1)$ & $11(1)$ \\
\hline
\end{tabular}




$\begin{array}{lllllll}\mathrm{C}(23) & 34(1) & 45(1) & 42(1) & -19(1) & -17(1) & 6(1) \\ \mathrm{C}(24) & 33(1) & 31(1) & 42(1) & -13(1) & -8(1) & -2(1) \\ \mathrm{C}(25) & 36(1) & 24(1) & 27(1) & -8(1) & -6(1) & 3(1)\end{array}$

Table 5. Hydrogen coordinates ( $\times 10^{4}$ ) and isotropic displacement parameters $\left(\AA^{2} \times 10^{3}\right)$ for $\mathbf{S}-23$.

\begin{tabular}{|c|c|c|c|c|}
\hline & $\mathrm{x}$ & $\mathrm{y}$ & $\mathrm{z}$ & $\mathrm{U}(\mathrm{eq})$ \\
\hline $\mathrm{H}(1)$ & $-2820(20)$ & $-971(16)$ & $-2016(15)$ & $38(5)$ \\
\hline $\mathrm{H}(2 \mathrm{~A})$ & $-630(20)$ & $4274(14)$ & $2212(15)$ & $31(5)$ \\
\hline $\mathrm{H}(2 \mathrm{~B})$ & $130(20)$ & $4805(14)$ & $1061(14)$ & $27(4)$ \\
\hline $\mathrm{H}(3 \mathrm{~A})$ & 2087(19) & $4060(14)$ & $2845(13)$ & $23(4)$ \\
\hline $\mathrm{H}(3 \mathrm{~B})$ & $1640(20)$ & $5345(15)$ & $2481(14)$ & $32(5)$ \\
\hline $\mathrm{H}(4 \mathrm{~A})$ & $3310(20)$ & $5193(14)$ & $787(13)$ & $24(4)$ \\
\hline $\mathrm{H}(5 \mathrm{~A})$ & 4132(19) & $2827(13)$ & $1412(13)$ & $21(4)$ \\
\hline $\mathrm{H}(5 \mathrm{~B})$ & $4730(20)$ & $3440(14)$ & $290(14)$ & $28(4)$ \\
\hline $\mathrm{H}(6 \mathrm{~A})$ & $1970(20)$ & $3560(14)$ & $-296(14)$ & $26(4)$ \\
\hline $\mathrm{H}(6 \mathrm{~B})$ & $2435(19)$ & $2254(14)$ & $110(13)$ & $24(4)$ \\
\hline $\mathrm{H}(7 \mathrm{~A})$ & $-1110(20)$ & $3373(15)$ & $77(15)$ & $31(5)$ \\
\hline $\mathrm{H}(7 \mathrm{~B})$ & $-1920(20)$ & $2827(14)$ & $1204(14)$ & $31(5)$ \\
\hline $\mathrm{H}(8 \mathrm{~A})$ & $-40(20)$ & $1583(15)$ & $-519(16)$ & $38(5)$ \\
\hline $\mathrm{H}(8 \mathrm{~B})$ & $-520(20)$ & 981(15) & $661(14)$ & $30(4)$ \\
\hline $\mathrm{H}(9 \mathrm{~A})$ & $-3380(20)$ & $1256(14)$ & $512(15)$ & $33(5)$ \\
\hline $\mathrm{H}(9 \mathrm{~B})$ & $-2980(20)$ & $2088(16)$ & $-591(15)$ & $38(5)$ \\
\hline $\mathrm{H}(12)$ & $-3310(20)$ & $-2106(14)$ & $-3433(14)$ & $29(4)$ \\
\hline $\mathrm{H}(13)$ & $-4810(20)$ & $-2406(15)$ & $-4900(14)$ & $30(5)$ \\
\hline $\mathrm{H}(15)$ & $-7970(20)$ & $317(14)$ & $-4184(14)$ & $29(4)$ \\
\hline $\mathrm{H}(16)$ & $-6490(20)$ & $622(14)$ & $-2671(14)$ & $29(4)$ \\
\hline $\mathrm{H}(17 \mathrm{~A})$ & $-460(30)$ & 2783(19) & $3387(18)$ & $54(6)$ \\
\hline $\mathrm{H}(17 \mathrm{~B})$ & $-1340(30)$ & $1907(18)$ & $2706(18)$ & $56(6)$ \\
\hline
\end{tabular}




$\begin{array}{lrlll}\mathrm{H}(17 \mathrm{C}) & 170(30) & 1441(17) & 3429(17) & 48(6) \\ \mathrm{H}(18 \mathrm{~A}) & 5250(20) & 4214(14) & 2488(14) & 29(4) \\ \mathrm{H}(18 B) & 6020(20) & 4787(14) & 1389(14) & 27(4) \\ \mathrm{H}(19 \mathrm{~A}) & 3970(30) & 6020(16) & 2998(17) & 46(6) \\ \mathrm{H}(19 B) & 4810(20) & 6621(17) & 1884(16) & 42(5) \\ \mathrm{H}(21) & 6070(20) & 4907(15) & 4253(14) & 33(5) \\ \mathrm{H}(22) & 8350(30) & 5257(17) & 5189(18) & 50(6) \\ \mathrm{H}(23) & 10150(30) & 6758(17) & 4585(17) & 47(6) \\ \mathrm{H}(24) & 9710(20) & 7827(17) & 2974(16) & 42(5) \\ \mathrm{H}(25) & 7350(20) & 7506(15) & 2053(15) & 37(5)\end{array}$

Table 6. Torsion angles $\left[^{\circ}\right]$ for $\mathbf{S - 2 3}$.

\begin{tabular}{lc}
\hline $\mathrm{C}(17)-\mathrm{O}(1)-\mathrm{C}(1)-\mathrm{C}(6)$ & $178.15(14)$ \\
$\mathrm{C}(17)-\mathrm{O}(1)-\mathrm{C}(1)-\mathrm{C}(7)$ & $-61.32(17)$ \\
$\mathrm{C}(17)-\mathrm{O}(1)-\mathrm{C}(1)-\mathrm{C}(2)$ & $60.68(17)$ \\
$\mathrm{O}(1)-\mathrm{C}(1)-\mathrm{C}(2)-\mathrm{C}(3)$ & $59.41(17)$ \\
$\mathrm{C}(6)-\mathrm{C}(1)-\mathrm{C}(2)-\mathrm{C}(3)$ & $-54.80(16)$ \\
$\mathrm{C}(7)-\mathrm{C}(1)-\mathrm{C}(2)-\mathrm{C}(3)$ & $-178.51(13)$ \\
$\mathrm{C}(1)-\mathrm{C}(2)-\mathrm{C}(3)-\mathrm{C}(4)$ & $56.42(17)$ \\
$\mathrm{C}(2)-\mathrm{C}(3)-\mathrm{C}(4)-\mathrm{C}(18)$ & $-175.40(12)$ \\
$\mathrm{C}(2)-\mathrm{C}(3)-\mathrm{C}(4)-\mathrm{C}(5)$ & $-52.58(16)$ \\
$\mathrm{C}(18)-\mathrm{C}(4)-\mathrm{C}(5)-\mathrm{C}(6)$ & $175.16(13)$ \\
$\mathrm{C}(3)-\mathrm{C}(4)-\mathrm{C}(5)-\mathrm{C}(6)$ & $50.87(17)$ \\
$\mathrm{C}(4)-\mathrm{C}(5)-\mathrm{C}(6)-\mathrm{C}(1)$ & $-52.41(17)$ \\
$\mathrm{O}(1)-\mathrm{C}(1)-\mathrm{C}(6)-\mathrm{C}(5)$ & $-65.47(15)$ \\
$\mathrm{C}(7)-\mathrm{C}(1)-\mathrm{C}(6)-\mathrm{C}(5)$ & $175.14(12)$ \\
$\mathrm{C}(2)-\mathrm{C}(1)-\mathrm{C}(6)-\mathrm{C}(5)$ & $52.52(16)$ \\
$\mathrm{O}(1)-\mathrm{C}(1)-\mathrm{C}(7)-\mathrm{C}(8)$ & $-57.33(18)$ \\
$\mathrm{C}(6)-\mathrm{C}(1)-\mathrm{C}(7)-\mathrm{C}(8)$ & $58.45(18)$ \\
$\mathrm{C}(2)-\mathrm{C}(1)-\mathrm{C}(7)-\mathrm{C}(8)$ & $-179.34(14)$ \\
$\mathrm{C}(1)-\mathrm{C}(7)-\mathrm{C}(8)-\mathrm{C}(9)$ & $171.74(14)$ \\
$\mathrm{C}(10)-\mathrm{O}(2)-\mathrm{C}(9)-\mathrm{C}(8)$ & $-168.47(13)$ \\
$\mathrm{C}(7)-\mathrm{C}(8)-\mathrm{C}(9)-\mathrm{O}(2)$ & $168.11(14)$ \\
& \\
&
\end{tabular}




$\begin{array}{lc}\mathrm{C}(9)-\mathrm{O}(2)-\mathrm{C}(10)-\mathrm{O}(3) & -3.4(2) \\ \mathrm{C}(9)-\mathrm{O}(2)-\mathrm{C}(10)-\mathrm{N}(1) & 176.66(13) \\ \mathrm{C}(11)-\mathrm{N}(1)-\mathrm{C}(10)-\mathrm{O}(3) & 17.0(2) \\ \mathrm{C}(11)-\mathrm{N}(1)-\mathrm{C}(10)-\mathrm{O}(2) & -163.14(13) \\ \mathrm{C}(10)-\mathrm{N}(1)-\mathrm{C}(11)-\mathrm{C}(12) & 175.60(14) \\ \mathrm{C}(10)-\mathrm{N}(1)-\mathrm{C}(11)-\mathrm{C}(16) & -1.6(2) \\ \mathrm{N}(1)-\mathrm{C}(11)-\mathrm{C}(12)-\mathrm{C}(13) & -176.06(14) \\ \mathrm{C}(16)-\mathrm{C}(11)-\mathrm{C}(12)-\mathrm{C}(13) & 1.3(2) \\ \mathrm{C}(11)-\mathrm{C}(12)-\mathrm{C}(13)-\mathrm{C}(14) & 0.2(2) \\ \mathrm{C}(12)-\mathrm{C}(13)-\mathrm{C}(14)-\mathrm{C}(15) & -1.0(2) \\ \mathrm{C}(12)-\mathrm{C}(13)-\mathrm{C}(14)-\mathrm{N}(2) & 178.44(13) \\ \mathrm{O}(5)-\mathrm{N}(2)-\mathrm{C}(14)-\mathrm{C}(15) & 179.47(15) \\ \mathrm{O}(4)-\mathrm{N}(2)-\mathrm{C}(14)-\mathrm{C}(15) & 0.5(2) \\ \mathrm{O}(5)-\mathrm{N}(2)-\mathrm{C}(14)-\mathrm{C}(13) & 0.0(2) \\ \mathrm{O}(4)-\mathrm{N}(2)-\mathrm{C}(14)-\mathrm{C}(13) & -178.92(14) \\ \mathrm{C}(13)-\mathrm{C}(14)-\mathrm{C}(15)-\mathrm{C}(16) & 0.3(2) \\ \mathrm{N}(2)-\mathrm{C}(14)-\mathrm{C}(15)-\mathrm{C}(16) & -179.09(14) \\ \mathrm{C}(14)-\mathrm{C}(15)-\mathrm{C}(16)-\mathrm{C}(11) & 1.1(2) \\ \mathrm{N}(1)-\mathrm{C}(11)-\mathrm{C}(16)-\mathrm{C}(15) & 175.26(14) \\ \mathrm{C}(12)-\mathrm{C}(11)-\mathrm{C}(16)-\mathrm{C}(15) & -1.9(2) \\ \mathrm{C}(3)-\mathrm{C}(4)-\mathrm{C}(18)-\mathrm{C}(19) & -58.78(18) \\ \mathrm{C}(5)-\mathrm{C}(4)-\mathrm{C}(18)-\mathrm{C}(19) & 178.25(14) \\ \mathrm{C}(4)-\mathrm{C}(18)-\mathrm{C}(19)-\mathrm{C}(20) & 176.39(14) \\ \mathrm{C}(18)-\mathrm{C}(19)-\mathrm{C}(20)-\mathrm{C}(25) & 115.05(17) \\ \mathrm{C}(18)-\mathrm{C}(19)-\mathrm{C}(20)-\mathrm{C}(21) & -66.3(2) \\ \mathrm{C}(25)-\mathrm{C}(20)-\mathrm{C}(21)-\mathrm{C}(22) & 1.0(2) \\ \mathrm{C}(19)-\mathrm{C}(20)-\mathrm{C}(21)-\mathrm{C}(22) & -177.68(15) \\ \mathrm{C}(20)-\mathrm{C}(21)-\mathrm{C}(22)-\mathrm{C}(23) & -0.6(3) \\ \mathrm{C}(21)-\mathrm{C}(22)-\mathrm{C}(23)-\mathrm{C}(24) & -0.1(3) \\ \mathrm{C}(22)-\mathrm{C}(23)-\mathrm{C}(24)-\mathrm{C}(25) & 0.5(3) \\ \mathrm{C}(23)-\mathrm{C}(24)-\mathrm{C}(25)-\mathrm{C}(20) & -0.1(3) \\ \mathrm{C}(21)-\mathrm{C}(20)-\mathrm{C}(25)-\mathrm{C}(24) & -0.6(2) \\ \mathrm{C}(19)-\mathrm{C}(20)-\mathrm{C}(25)-\mathrm{C}(24) & 178.04(15) \\ & \\ & \\ & \end{array}$




\section{Carbamate S-25}

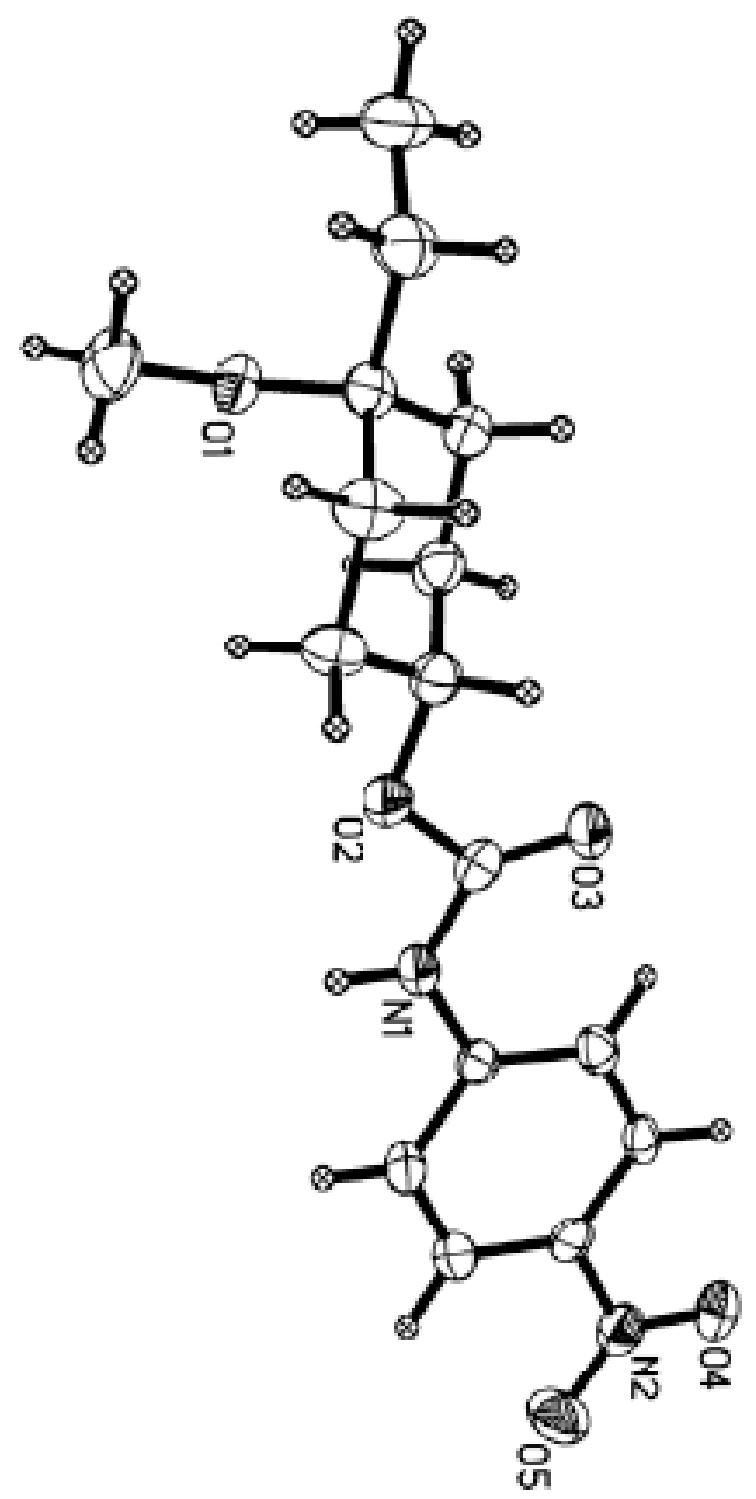

The colorless crystals of $\mathrm{C}_{16} \mathrm{H}_{22} \mathrm{~N}_{2} \mathrm{O}_{5}$ are monoclinic, space group $\mathrm{P} 2_{1} / \mathrm{c}$, with $\mathrm{a}=$ 14.125(8), $\mathrm{b}=11.847(7), \mathrm{c}=19.754(9) \AA, \beta=98.78(2)^{\circ}, \mathrm{V}=3267(5) \AA^{3}, \mathrm{Z}=8$. Data were collected on a Bruker SMART-CCD diffractometer with MoK $\alpha$ radiation at $\mathrm{T}=$ $171 \mathrm{~K}$. Structure solution and refinement were carried out by use of the SHELXTL program system. Final agreement factors, based on 4254 data with $|F| \geq 2 \sigma(F)$, were R1 $=0.050$ and $\mathrm{wR} 2=0.127$, and $\mathrm{GOF}=1.021$. Full details are available in the deposited .cif file.

Table 1. Crystal data and structure refinement for $\mathbf{S - 2 5}$. 
Empirical formula

Formula weight

Temperature

Wavelength

Crystal system

Space group

Unit cell dimensions

Volume

Z

Density (calculated)

Absorption coefficient

$\mathrm{F}(000)$

Crystal size

Theta range for data collection

Index ranges

Reflections collected

Independent reflections

Completeness to theta $=28.30^{\circ}$

Absorption correction

Max. and min. transmission

Refinement method

Data / restraints / parameters

Goodness-of-fit on $\mathrm{F}^{2}$

Final $\mathrm{R}$ indices [I $>2 \operatorname{sigma}(\mathrm{I})]$

$\mathrm{R}$ indices (all data)

Largest diff. peak and hole
$\mathrm{C}_{16} \mathrm{H}_{22} \mathrm{~N}_{2} \mathrm{O}_{5}$

322.36

171(2) K

$0.71073 \AA$

Monoclinic

$\mathrm{P} 2(1) / \mathrm{c}$

$$
\begin{array}{ll}
\mathrm{a}=14.1248(18) \AA & \alpha=90^{\circ} . \\
\mathrm{b}=11.8471(15) \AA & \beta=98.782(2)^{\circ} . \\
\mathrm{c}=19.754(3) \AA & \gamma=90^{\circ} .
\end{array}
$$

$3266.9(7) \AA^{3}$

8

$1.311 \mathrm{Mg} / \mathrm{m}^{3}$

$0.098 \mathrm{~mm}^{-1}$

1376

$0.37 \times 0.32 \times 0.09 \mathrm{~mm}^{3}$

1.46 to $28.30^{\circ}$.

$-18 \leq \mathrm{h} \leq 18,-15 \leq \mathrm{k} \leq 15,-26 \leq 1 \leq 26$

34170

$7893[\mathrm{R}($ int $)=0.0725]$

$97.2 \%$

Semi-empirical from equivalents

1.00 and 0.84

Full-matrix least-squares on $\mathrm{F}^{2}$

7893 / 0 / 419

1.021

$\mathrm{R} 1=0.0500, \mathrm{wR} 2=0.1271$

$\mathrm{R} 1=0.1304, \mathrm{wR} 2=0.1819$

0.319 and $-0.344 \mathrm{e}^{-}-3$

Table 2. Atomic coordinates $\left(\times 10^{4}\right)$ and equivalent isotropic displacement parameters $\left(\AA^{2} \times 10^{3}\right)$ for 1 . U(eq) is defined as one third of the trace of the orthogonalized Uij tensor. 
$\begin{array}{lllll}\mathrm{x} & \mathrm{y} & \mathrm{z} & \mathrm{U}(\mathrm{eq})\end{array}$

\begin{tabular}{|c|c|c|c|c|}
\hline $\mathrm{O}(1)$ & $3642(1)$ & $-1166(1)$ & $3042(1)$ & $27(1)$ \\
\hline $\mathrm{O}(2)$ & 1393(1) & $113(2)$ & 1391(1) & $32(1)$ \\
\hline $\mathrm{O}(3)$ & 1681(1) & $1604(2)$ & $729(1)$ & $40(1)$ \\
\hline $\mathrm{O}(4)$ & $-1878(1)$ & $5096(2)$ & $-877(1)$ & $38(1)$ \\
\hline $\mathrm{O}(5)$ & $-3106(1)$ & $4145(2)$ & $-668(1)$ & $50(1)$ \\
\hline $\mathrm{O}(21)$ & $-915(1)$ & $-607(1)$ & $1496(1)$ & $23(1)$ \\
\hline $\mathrm{O}(22)$ & 1341(1) & $-488(1)$ & $3312(1)$ & $24(1)$ \\
\hline $\mathrm{O}(23)$ & $1165(1)$ & $697(1)$ & 4184(1) & $27(1)$ \\
\hline $\mathrm{O}(24)$ & 5913(1) & $3600(2)$ & $5119(1)$ & $42(1)$ \\
\hline $\mathrm{O}(25)$ & 4801(1) & $4161(2)$ & $5686(1)$ & $46(1)$ \\
\hline $\mathrm{N}(1)$ & $170(1)$ & $1174(2)$ & $953(1)$ & $28(1)$ \\
\hline $\mathrm{N}(2)$ & $-2238(2)$ & $4298(2)$ & $-612(1)$ & $31(1)$ \\
\hline $\mathrm{N}(21)$ & 2641(1) & $264(2)$ & $3895(1)$ & $24(1)$ \\
\hline $\mathrm{N}(22)$ & $5101(2)$ & $3528(2)$ & $5273(1)$ & $31(1)$ \\
\hline $\mathrm{C}(1)$ & $4068(2)$ & $-1127(2)$ & $2417(1)$ & $28(1)$ \\
\hline$C(2)$ & $4002(2)$ & $116(2)$ & $2215(1)$ & $31(1)$ \\
\hline$C(3)$ & $2976(2)$ & $545(2)$ & 2031(1) & $32(1)$ \\
\hline $\mathrm{C}(4)$ & $2408(2)$ & $-191(2)$ & $1486(1)$ & $31(1)$ \\
\hline$C(5)$ & $2446(2)$ & $-1416(2)$ & $1696(1)$ & $33(1)$ \\
\hline$C(6)$ & $3481(2)$ & $-1820(2)$ & $1852(1)$ & $37(1)$ \\
\hline$C(7)$ & $5105(2)$ & $-1551(3)$ & $2556(1)$ & $37(1)$ \\
\hline$C(8)$ & $5747(2)$ & $-996(3)$ & $3159(1)$ & $46(1)$ \\
\hline$C(9)$ & $3499(2)$ & $-2263(2)$ & $3307(2)$ & $48(1)$ \\
\hline$C(10)$ & $1136(2)$ & $1026(2)$ & 993(1) & $30(1)$ \\
\hline $\mathrm{C}(11)$ & $-391(2)$ & $1975(2)$ & $557(1)$ & $24(1)$ \\
\hline$C(12)$ & $-1391(2)$ & $1857(2)$ & $499(1)$ & $30(1)$ \\
\hline$C(13)$ & $-1997(2)$ & $2610(2)$ & $118(1)$ & $30(1)$ \\
\hline$C(14)$ & $-1601(2)$ & $3492(2)$ & $-205(1)$ & $25(1)$ \\
\hline$C(15)$ & $-623(2)$ & $3628(2)$ & $-156(1)$ & $26(1)$ \\
\hline$C(16)$ & $-15(2)$ & $2876(2)$ & $224(1)$ & $26(1)$ \\
\hline$C(21)$ & $-1390(2)$ & $-912(2)$ & 2073(1) & $22(1)$ \\
\hline$C(22)$ & $-1249(2)$ & $131(2)$ & $2532(1)$ & $23(1)$ \\
\hline$C(23)$ & $-192(2)$ & $366(2)$ & $2803(1)$ & $23(1)$ \\
\hline
\end{tabular}


Supporting Information: Glen Baghdasarian and K. A. Woerpel

$\begin{array}{lrrrr}\mathrm{C}(24) & 300(2) & -655(2) & 3161(1) & 22(1) \\ \mathrm{C}(25) & 171(2) & -1701(2) & 2712(1) & 25(1) \\ \mathrm{C}(26) & -890(2) & -1915(2) & 2468(1) & 25(1) \\ \mathrm{C}(27) & -2445(2) & -1200(2) & 1838(1) & 28(1) \\ \mathrm{C}(28) & -3014(2) & -345(2) & 1367(2) & 41(1) \\ \mathrm{C}(29) & -849(2) & -1463(2) & 999(1) & 39(1) \\ \mathrm{C}(30) & 1668(2) & 211(2) & 3833(1) & 22(1) \\ \mathrm{C}(31) & 3235(2) & 1047(2) & 4293(1) & 22(1) \\ \mathrm{C}(32) & 4179(2) & 1106(2) & 4176(1) & 30(1) \\ \mathrm{C}(33) & 4802(2) & 1902(2) & 4502(1) & 30(1) \\ \mathrm{C}(34) & 4463(2) & 2641(2) & 4953(1) & 24(1) \\ \mathrm{C}(35) & 3545(2) & 2566(2) & 5103(1) & 25(1) \\ \mathrm{C}(36) & 2928(2) & 1764(2) & 4777(1) & 23(1)\end{array}$

Table 3. Bond lengths $[\AA]$ and angles $\left[{ }^{\circ}\right]$ for $\mathbf{S - 2 5}$.

\begin{tabular}{ll}
\hline $\mathrm{O}(1)-\mathrm{C}(9)$ & $1.427(3)$ \\
$\mathrm{O}(1)-\mathrm{C}(1)$ & $1.454(3)$ \\
$\mathrm{O}(2)-\mathrm{C}(10)$ & $1.353(3)$ \\
$\mathrm{O}(2)-\mathrm{C}(4)$ & $1.462(3)$ \\
$\mathrm{O}(3)-\mathrm{C}(10)$ & $1.207(3)$ \\
$\mathrm{O}(4)-\mathrm{N}(2)$ & $1.227(3)$ \\
$\mathrm{O}(5)-\mathrm{N}(2)$ & $1.227(3)$ \\
$\mathrm{O}(21)-\mathrm{C}(29)$ & $1.425(3)$ \\
$\mathrm{O}(21)-\mathrm{C}(21)$ & $1.453(3)$ \\
$\mathrm{O}(22)-\mathrm{C}(30)$ & $1.347(3)$ \\
$\mathrm{O}(22)-\mathrm{C}(24)$ & $1.468(3)$ \\
$\mathrm{O}(23)-\mathrm{C}(30)$ & $1.210(3)$ \\
$\mathrm{O}(24)-\mathrm{N}(22)$ & $1.233(3)$ \\
$\mathrm{O}(25)-\mathrm{N}(22)$ & $1.230(3)$ \\
$\mathrm{N}(1)-\mathrm{C}(10)$ & $1.366(3)$ \\
$\mathrm{N}(1)-\mathrm{C}(11)$ & $1.396(3)$ \\
$\mathrm{N}(2)-\mathrm{C}(14)$ & $1.466(3)$ \\
$\mathrm{N}(21)-\mathrm{C}(30)$ & $1.363(3)$
\end{tabular}




$\begin{array}{lr}\mathrm{N}(21)-\mathrm{C}(31) & 1.408(3) \\ \mathrm{N}(22)-\mathrm{C}(34) & 1.464(3) \\ \mathrm{C}(1)-\mathrm{C}(6) & 1.524(3) \\ \mathrm{C}(1)-\mathrm{C}(2) & 1.524(4) \\ \mathrm{C}(1)-\mathrm{C}(7) & 1.533(4) \\ \mathrm{C}(2)-\mathrm{C}(3) & 1.526(3) \\ \mathrm{C}(3)-\mathrm{C}(4) & 1.516(3) \\ \mathrm{C}(4)-\mathrm{C}(5) & 1.508(4) \\ \mathrm{C}(5)-\mathrm{C}(6) & 1.525(4) \\ \mathrm{C}(7)-\mathrm{C}(8) & 1.531(4) \\ \mathrm{C}(11)-\mathrm{C}(16) & 1.400(3) \\ \mathrm{C}(11)-\mathrm{C}(12) & 1.406(3) \\ \mathrm{C}(12)-\mathrm{C}(13) & 1.378(3) \\ \mathrm{C}(13)-\mathrm{C}(14) & 1.385(3) \\ \mathrm{C}(14)-\mathrm{C}(15) & 1.380(3) \\ \mathrm{C}(15)-\mathrm{C}(16) & 1.379(3) \\ \mathrm{C}(21)-\mathrm{C}(27) & 1.530(3) \\ \mathrm{C}(21)-\mathrm{C}(22) & 1.528(3) \\ \mathrm{C}(21)-\mathrm{C}(26) & 1.534(3) \\ \mathrm{C}(22)-\mathrm{C}(23) & 1.533(3) \\ \mathrm{C}(23)-\mathrm{C}(24) & 1.515(3) \\ \mathrm{C}(24)-\mathrm{C}(25) & 1.518(3) \\ \mathrm{C}(25)-\mathrm{C}(26) & 1.524(3) \\ \mathrm{C}(27)-\mathrm{C}(28) & 1.520(3) \\ \mathrm{C}(31)-\mathrm{C}(32) & 1.391(3) \\ \mathrm{C}(31)-\mathrm{C}(36) & 1.395(3) \\ \mathrm{C}(32)-\mathrm{C}(33) & 1.379(3) \\ \mathrm{C}(33)-\mathrm{C}(34) & 1.386(3) \\ \mathrm{C}(34)-\mathrm{C}(35) & 1.376(3) \\ \mathrm{C}(35)-\mathrm{C}(36) & 1.382(3) \\ \mathrm{C}(9)-\mathrm{O}(1)-\mathrm{C}(1) & 116.1(2) \\ \mathrm{C}(10)-\mathrm{O}(2)-\mathrm{C}(4) & 116.28(19) \\ \mathrm{C}(29)-\mathrm{O}(21)-\mathrm{C}(21) & 116.71(18) \\ \mathrm{C}(30)-\mathrm{O}(22)-\mathrm{C}(24) & 116.99(17) \\ \mathrm{C}(10)-\mathrm{N}(1)-\mathrm{C}(11) & 126.6(2) \\ \mathrm{O}(5)-\mathrm{N}(2)-\mathrm{O}(4) & 123.3(2) \\ & \end{array}$




$\begin{array}{ll}\mathrm{O}(5)-\mathrm{N}(2)-\mathrm{C}(14) & 118.3(2) \\ \mathrm{O}(4)-\mathrm{N}(2)-\mathrm{C}(14) & 118.5(2) \\ \mathrm{C}(30)-\mathrm{N}(21)-\mathrm{C}(31) & 125.90(19) \\ \mathrm{O}(24)-\mathrm{N}(22)-\mathrm{O}(25) & 123.3(2) \\ \mathrm{O}(24)-\mathrm{N}(22)-\mathrm{C}(34) & 118.5(2) \\ \mathrm{O}(25)-\mathrm{N}(22)-\mathrm{C}(34) & 118.1(2) \\ \mathrm{O}(1)-\mathrm{C}(1)-\mathrm{C}(6) & 110.8(2) \\ \mathrm{O}(1)-\mathrm{C}(1)-\mathrm{C}(2) & 103.61(19) \\ \mathrm{C}(6)-\mathrm{C}(1)-\mathrm{C}(2) & 108.9(2) \\ \mathrm{O}(1)-\mathrm{C}(1)-\mathrm{C}(7) & 110.2(2) \\ \mathrm{C}(6)-\mathrm{C}(1)-\mathrm{C}(7) & 110.7(2) \\ \mathrm{C}(2)-\mathrm{C}(1)-\mathrm{C}(7) & 112.5(2) \\ \mathrm{C}(1)-\mathrm{C}(2)-\mathrm{C}(3) & 113.7(2) \\ \mathrm{C}(4)-\mathrm{C}(3)-\mathrm{C}(2) & 111.0(2) \\ \mathrm{O}(2)-\mathrm{C}(4)-\mathrm{C}(5) & 105.4(2) \\ \mathrm{O}(2)-\mathrm{C}(4)-\mathrm{C}(3) & 110.6(2) \\ \mathrm{C}(5)-\mathrm{C}(4)-\mathrm{C}(3) & 111.4(2) \\ \mathrm{C}(4)-\mathrm{C}(5)-\mathrm{C}(6) & 110.5(2) \\ \mathrm{C}(5)-\mathrm{C}(6)-\mathrm{C}(1) & 112.2(2) \\ \mathrm{C}(1)-\mathrm{C}(7)-\mathrm{C}(8) & 115.8(2) \\ \mathrm{O}(3)-\mathrm{C}(10)-\mathrm{O}(2) & 124.8(2) \\ \mathrm{O}(3)-\mathrm{C}(10)-\mathrm{N}(1) & 126.8(2) \\ \mathrm{O}(2)-\mathrm{C}(10)-\mathrm{N}(1) & 108.3(2) \\ \mathrm{C}(16)-\mathrm{C}(11)-\mathrm{N}(1) & 123.9(2) \\ \mathrm{C}(16)-\mathrm{C}(11)-\mathrm{C}(12) & 119.1(2) \\ \mathrm{N}(1)-\mathrm{C}(11)-\mathrm{C}(12) & 117.1(2) \\ \mathrm{C}(13)-\mathrm{C}(12)-\mathrm{C}(11) & 120.9(2) \\ \mathrm{C}(12)-\mathrm{C}(13)-\mathrm{C}(14) & 118.5(2) \\ \mathrm{C}(15)-\mathrm{C}(14)-\mathrm{C}(13) & 121.8(2) \\ \mathrm{C}(15)-\mathrm{C}(14)-\mathrm{N}(2) & 119.0(2) \\ \mathrm{C}(13)-\mathrm{C}(14)-\mathrm{N}(2) & 119.1(2) \\ \mathrm{C}(16)-\mathrm{C}(15)-\mathrm{C}(14) & 119.7(2) \\ \mathrm{C}(15)-\mathrm{C}(16)-\mathrm{C}(11) & 119.9(2) \\ \mathrm{O}(21)-\mathrm{C}(21)-\mathrm{C}(27) & 111.27(18) \\ \mathrm{O}(21)-\mathrm{C}(21)-\mathrm{C}(22) & 103.34(17) \\ \mathrm{C}(27)-\mathrm{C}(21)-\mathrm{C}(22) & 113.09(19) \\ & \end{array}$




$\begin{array}{ll}\mathrm{O}(21)-\mathrm{C}(21)-\mathrm{C}(26) & 111.12(19) \\ \mathrm{C}(27)-\mathrm{C}(21)-\mathrm{C}(26) & 109.43(18) \\ \mathrm{C}(22)-\mathrm{C}(21)-\mathrm{C}(26) & 108.47(18) \\ \mathrm{C}(21)-\mathrm{C}(22)-\mathrm{C}(23) & 112.70(19) \\ \mathrm{C}(24)-\mathrm{C}(23)-\mathrm{C}(22) & 111.75(19) \\ \mathrm{O}(22)-\mathrm{C}(24)-\mathrm{C}(23) & 111.16(18) \\ \mathrm{O}(22)-\mathrm{C}(24)-\mathrm{C}(25) & 104.84(17) \\ \mathrm{C}(23)-\mathrm{C}(24)-\mathrm{C}(25) & 111.97(18) \\ \mathrm{C}(24)-\mathrm{C}(25)-\mathrm{C}(26) & 110.24(19) \\ \mathrm{C}(25)-\mathrm{C}(26)-\mathrm{C}(21) & 112.72(19) \\ \mathrm{C}(28)-\mathrm{C}(27)-\mathrm{C}(21) & 115.9(2) \\ \mathrm{O}(23)-\mathrm{C}(30)-\mathrm{O}(22) & 124.6(2) \\ \mathrm{O}(23)-\mathrm{C}(30)-\mathrm{N}(21) & 126.8(2) \\ \mathrm{O}(22)-\mathrm{C}(30)-\mathrm{N}(21) & 108.61(19) \\ \mathrm{C}(32)-\mathrm{C}(31)-\mathrm{C}(36) & 119.3(2) \\ \mathrm{C}(32)-\mathrm{C}(31)-\mathrm{N}(21) & 116.4(2) \\ \mathrm{C}(36)-\mathrm{C}(31)-\mathrm{N}(21) & 124.3(2) \\ \mathrm{C}(33)-\mathrm{C}(32)-\mathrm{C}(31) & 121.1(2) \\ \mathrm{C}(32)-\mathrm{C}(33)-\mathrm{C}(34) & 118.3(2) \\ \mathrm{C}(35)-\mathrm{C}(34)-\mathrm{C}(33) & 121.8(2) \\ \mathrm{C}(35)-\mathrm{C}(34)-\mathrm{N}(22) & 119.4(2) \\ \mathrm{C}(33)-\mathrm{C}(34)-\mathrm{N}(22) & 118.8(2) \\ \mathrm{C}(34)-\mathrm{C}(35)-\mathrm{C}(36) & 119.5(2) \\ \mathrm{C}(35)-\mathrm{C}(36)-\mathrm{C}(31) & 119.9(2) \\ & \end{array}$

Table 4. Anisotropic displacement parameters $\left(\AA^{2} \times 10^{3}\right)$ for S-25. The anisotropic displacement factor exponent takes the form: $-2 \pi^{2}\left[h^{2} a^{* 2} U^{11}+\ldots+2 h k a^{*} b^{*} U^{12}\right]$
$\mathrm{U}^{11}$
$\mathrm{U}^{22}$
$\mathrm{U}^{33}$
$\mathrm{U}^{23}$
$\mathrm{U}^{13}$
$\mathrm{U}^{12}$ 


\begin{tabular}{|c|c|c|c|c|c|c|}
\hline $\mathrm{O}(1)$ & $34(1)$ & $23(1)$ & $26(1)$ & $4(1)$ & $11(1)$ & $5(1)$ \\
\hline $\mathrm{O}(2)$ & $28(1)$ & $38(1)$ & $30(1)$ & $7(1)$ & $7(1)$ & $2(1)$ \\
\hline $\mathrm{O}(3)$ & $27(1)$ & $57(1)$ & $38(1)$ & $21(1)$ & $9(1)$ & $-2(1)$ \\
\hline $\mathrm{O}(4)$ & $47(1)$ & $37(1)$ & $32(1)$ & $10(1)$ & $12(1)$ & $-2(1)$ \\
\hline $\mathrm{O}(5)$ & $30(1)$ & $52(1)$ & $66(1)$ & $20(1)$ & $-3(1)$ & $-2(1)$ \\
\hline $\mathrm{O}(21)$ & $30(1)$ & $21(1)$ & $20(1)$ & $-3(1)$ & $7(1)$ & $-4(1)$ \\
\hline $\mathrm{O}(22)$ & $23(1)$ & $26(1)$ & $23(1)$ & $-5(1)$ & $2(1)$ & $-2(1)$ \\
\hline $\mathrm{O}(23)$ & $26(1)$ & $30(1)$ & $25(1)$ & $-5(1)$ & $8(1)$ & $-1(1)$ \\
\hline $\mathrm{O}(24)$ & $26(1)$ & $44(1)$ & $55(1)$ & $-12(1)$ & $4(1)$ & $-6(1)$ \\
\hline $\mathrm{O}(25)$ & $39(1)$ & $48(1)$ & $48(1)$ & $-27(1)$ & $5(1)$ & $-4(1)$ \\
\hline $\mathrm{N}(1)$ & $25(1)$ & $33(1)$ & $26(1)$ & $6(1)$ & $8(1)$ & $-2(1)$ \\
\hline $\mathrm{N}(2)$ & $37(1)$ & $32(1)$ & $25(1)$ & $0(1)$ & $6(1)$ & $-1(1)$ \\
\hline $\mathrm{N}(21)$ & $19(1)$ & $28(1)$ & $26(1)$ & $-7(1)$ & $6(1)$ & $0(1)$ \\
\hline $\mathrm{N}(22)$ & $28(1)$ & $32(1)$ & $32(1)$ & $-5(1)$ & $-1(1)$ & $0(1)$ \\
\hline $\mathrm{C}(1)$ & $29(1)$ & $33(1)$ & $24(1)$ & $-6(1)$ & $9(1)$ & $2(1)$ \\
\hline$C(2)$ & $30(2)$ & $37(2)$ & $26(1)$ & $4(1)$ & $3(1)$ & $-5(1)$ \\
\hline$C(3)$ & $37(2)$ & $27(1)$ & $31(1)$ & $5(1)$ & $3(1)$ & $-2(1)$ \\
\hline$C(4)$ & $28(1)$ & $42(2)$ & $23(1)$ & $0(1)$ & $5(1)$ & $1(1)$ \\
\hline$C(5)$ & $30(2)$ & $33(2)$ & $34(1)$ & $-11(1)$ & $1(1)$ & $1(1)$ \\
\hline$C(6)$ & $37(2)$ & $39(2)$ & $37(2)$ & $-14(1)$ & $6(1)$ & $6(1)$ \\
\hline$C(7)$ & $34(2)$ & $45(2)$ & $33(1)$ & $-5(1)$ & $9(1)$ & $5(1)$ \\
\hline$C(8)$ & $31(2)$ & $62(2)$ & $42(2)$ & $-4(2)$ & $-2(1)$ & $7(2)$ \\
\hline$C(9)$ & $54(2)$ & $32(2)$ & $59(2)$ & $20(1)$ & $15(2)$ & $7(2)$ \\
\hline$C(10)$ & $33(2)$ & $38(2)$ & $19(1)$ & $2(1)$ & $5(1)$ & $-1(1)$ \\
\hline $\mathrm{C}(11)$ & $25(1)$ & $32(1)$ & $16(1)$ & $-3(1)$ & $7(1)$ & $-3(1)$ \\
\hline$C(12)$ & $32(2)$ & $33(1)$ & $26(1)$ & $5(1)$ & $8(1)$ & $-8(1)$ \\
\hline$C(13)$ & $26(1)$ & $38(2)$ & $26(1)$ & $1(1)$ & $7(1)$ & $-3(1)$ \\
\hline$C(14)$ & $32(1)$ & $25(1)$ & $17(1)$ & $-3(1)$ & $5(1)$ & $0(1)$ \\
\hline$C(15)$ & $31(1)$ & $25(1)$ & $23(1)$ & $-4(1)$ & $10(1)$ & $-6(1)$ \\
\hline$C(16)$ & $26(1)$ & $30(1)$ & $23(1)$ & $-3(1)$ & $9(1)$ & $-4(1)$ \\
\hline $\mathrm{C}(21)$ & $24(1)$ & $20(1)$ & $21(1)$ & $3(1)$ & $6(1)$ & $-2(1)$ \\
\hline$C(22)$ & $27(1)$ & $22(1)$ & $20(1)$ & $1(1)$ & $4(1)$ & $2(1)$ \\
\hline$C(23)$ & $32(1)$ & $18(1)$ & $19(1)$ & $0(1)$ & $4(1)$ & $-2(1)$ \\
\hline $\mathrm{C}(24)$ & $22(1)$ & $22(1)$ & $21(1)$ & $-1(1)$ & $4(1)$ & $-3(1)$ \\
\hline$C(25)$ & $26(1)$ & $18(1)$ & $30(1)$ & $1(1)$ & $2(1)$ & $2(1)$ \\
\hline$C(26)$ & $26(1)$ & $17(1)$ & $30(1)$ & $2(1)$ & $2(1)$ & $-3(1)$ \\
\hline
\end{tabular}


Supporting Information: Glen Baghdasarian and K. A. Woerpel

$\begin{array}{llllccc}\mathrm{C}(27) & 24(1) & 25(1) & 35(1) & 0(1) & 2(1) & -2(1) \\ \mathrm{C}(28) & 32(2) & 33(2) & 53(2) & 1(1) & -12(1) & 2(1) \\ \mathrm{C}(29) & 50(2) & 35(2) & 32(1) & -15(1) & 11(1) & -7(1) \\ \mathrm{C}(30) & 27(1) & 21(1) & 18(1) & 1(1) & 4(1) & 0(1) \\ \mathrm{C}(31) & 21(1) & 25(1) & 20(1) & 0(1) & 0(1) & 2(1) \\ \mathrm{C}(32) & 26(1) & 34(1) & 31(1) & -12(1) & 7(1) & 1(1) \\ \mathrm{C}(33) & 20(1) & 42(2) & 30(1) & -8(1) & 6(1) & -2(1) \\ \mathrm{C}(34) & 22(1) & 25(1) & 22(1) & -2(1) & -2(1) & 0(1) \\ \mathrm{C}(35) & 26(1) & 27(1) & 20(1) & -3(1) & 2(1) & 5(1) \\ \mathrm{C}(36) & 22(1) & 27(1) & 21(1) & 0(1) & 5(1) & 2(1)\end{array}$

Table 5. Hydrogen coordinates ( $\times 10^{4}$ ) and isotropic displacement parameters $\left(\AA^{2} \times 10^{3}\right)$ for $\mathbf{S}-\mathbf{2 5}$.

$\begin{array}{lllll}\mathrm{x} & \mathrm{y} & \mathrm{z} & \mathrm{U}(\mathrm{eq})\end{array}$

$\begin{array}{lrrrr}\mathrm{H}(1) & -114 & 630 & 1169 & 33 \\ \mathrm{H}(21) & 2975 & -177 & 3571 & 29 \\ \mathrm{H}(2 \mathrm{~A}) & 4342 & 232 & 1818 & 37 \\ \mathrm{H}(2 \mathrm{~B}) & 4333 & 571 & 2601 & 37 \\ \mathrm{H}(3 \mathrm{~A}) & 2661 & 546 & 2445 & 38 \\ \mathrm{H}(3 \mathrm{~B}) & 2986 & 1330 & 1861 & 38 \\ \mathrm{H}(4) & 2662 & -103 & 1043 & 37 \\ \mathrm{H}(5 \mathrm{~A}) & 2094 & -1879 & 1322 & 39 \\ \mathrm{H}(5 \mathrm{~B}) & 2131 & -1512 & 2107 & 39 \\ \mathrm{H}(6 \mathrm{~A}) & 3779 & -1768 & 1431 & 45 \\ \mathrm{H}(6 \mathrm{~B}) & 3490 & -2622 & 1993 & 45 \\ \mathrm{H}(7 \mathrm{~A}) & 5399 & -1433 & 2138 & 44 \\ \mathrm{H}(7 \mathrm{~B}) & 5094 & -2375 & 2641 & 44 \\ \mathrm{H}(8 \mathrm{~A}) & 5843 & -199 & 3053 & 69 \\ \mathrm{H}(8 \mathrm{~B}) & 6367 & -1382 & 3238 & 69\end{array}$


Supporting Information: Glen Baghdasarian and K. A. Woerpel

\begin{tabular}{|c|c|c|c|}
\hline $\mathrm{H}(8 \mathrm{C})$ & 5440 & -1050 & 3571 \\
\hline $\mathrm{H}(9 \mathrm{~A})$ & 2994 & -2654 & 2999 \\
\hline $\mathrm{H}(9 \mathrm{~B})$ & 3309 & -2193 & 3762 \\
\hline $\mathrm{H}(9 \mathrm{C})$ & 4095 & -2696 & 3341 \\
\hline $\mathrm{H}(12)$ & -1652 & 1251 & 725 \\
\hline $\mathrm{H}(13)$ & -2673 & 2526 & 77 \\
\hline $\mathrm{H}(15)$ & -370 & 4238 & -384 \\
\hline $\mathrm{H}(16)$ & 659 & 2968 & 261 \\
\hline $\mathrm{H}(22 \mathrm{~A})$ & -1600 & 24 & 2925 \\
\hline $\mathrm{H}(22 \mathrm{~B})$ & -1527 & 795 & 2269 \\
\hline $\mathrm{H}(23 \mathrm{~A})$ & 141 & 585 & 2417 \\
\hline $\mathrm{H}(23 \mathrm{~B})$ & -146 & 1007 & 3128 \\
\hline $\mathrm{H}(24)$ & 40 & -799 & 3596 \\
\hline $\mathrm{H}(25 \mathrm{~A})$ & 455 & -2362 & 2976 \\
\hline $\mathrm{H}(25 \mathrm{~B})$ & 507 & -1598 & 2312 \\
\hline $\mathrm{H}(26 \mathrm{~A})$ & -1211 & -2077 & 2869 \\
\hline $\mathrm{H}(26 \mathrm{~B})$ & -959 & -2590 & 2169 \\
\hline $\mathrm{H}(27 \mathrm{~A})$ & -2478 & -1937 & 1600 \\
\hline $\mathrm{H}(27 \mathrm{~B})$ & -2759 & -1291 & 2250 \\
\hline $\mathrm{H}(28 \mathrm{~A})$ & -2967 & 399 & 1587 \\
\hline $\mathrm{H}(28 \mathrm{~B})$ & -3687 & -578 & 1275 \\
\hline $\mathrm{H}(28 \mathrm{C})$ & -2755 & -305 & 935 \\
\hline $\mathrm{H}(29 \mathrm{~A})$ & -511 & -2118 & 1222 \\
\hline $\mathrm{H}(29 \mathrm{~B})$ & -496 & -1172 & 646 \\
\hline $\mathrm{H}(29 \mathrm{C})$ & -1494 & -1689 & 788 \\
\hline $\mathrm{H}(32)$ & 4400 & 590 & 3867 \\
\hline $\mathrm{H}(33)$ & 5446 & 1944 & 4418 \\
\hline $\mathrm{H}(35)$ & 3338 & 3063 & 5429 \\
\hline $\mathrm{H}(36)$ & 2296 & 1700 & 4881 \\
\hline
\end{tabular}

\section{Amide S-26}




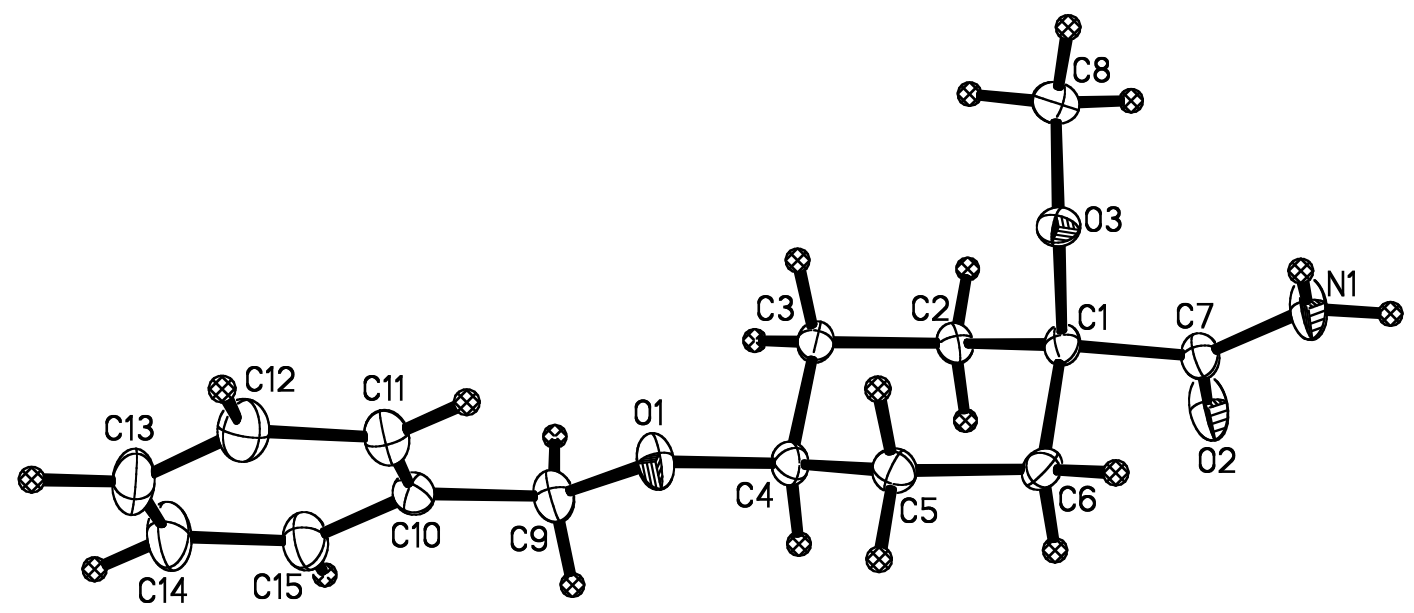

X-ray Data Collection, Structure Solution and Refinement for S-26.

A colorless crystal of approximate dimensions $0.25 \times 0.30 \times 0.43 \mathrm{~mm}$ was mounted on a glass fiber and transferred to a Bruker CCD platform diffractometer. The SMART ${ }^{1}$ program package was used to determine the unit-cell parameters and for data collection $(25 \mathrm{sec} /$ frame scan time for a sphere of diffraction data). The raw frame data was processed using SAINT ${ }^{2}$ and $\mathrm{SADABS}^{3}$ to yield the reflection data file. Subsequent calculations were carried out using the SHELXTL ${ }^{4}$ program. The diffraction symmetry was $2 / m$ and the systematic absences were consistent with the centrosymmetric monoclinic space group $P 2_{1} / c$ which was later determined to be correct.

The structure was solved by direct methods and refined on $\mathrm{F}^{2}$ by full-matrix least-squares techniques. The analytical scattering factors ${ }^{5}$ for neutral atoms were used throughout the analysis. Hydrogen atoms were located from a difference-Fourier map and refined (x,y,z and $\mathrm{U}_{\text {iso }}$ ). There were two molecules of the formula unit present. At convergence, wR2 $=0.1082$ and Goof $=1.085$ for 511 variables refined against 6853 data. As a comparison for refinement on F, R1 = 0.0421 for those 5559 data with $\mathrm{I}>2.0 \sigma(\mathrm{I})$.

Definitions:

$$
\begin{aligned}
& \mathrm{wR} 2=\left[\Sigma\left[\mathrm{w}\left(\mathrm{F}_{\mathrm{o}}{ }^{2}-\mathrm{F}_{\mathrm{c}}\right)^{2}\right] / \Sigma\left[\mathrm{w}\left(\mathrm{F}_{\mathrm{o}}{ }^{2}\right)^{2}\right]\right]^{1 / 2} \\
& \mathrm{R} 1=\Sigma|| \mathrm{F}_{\mathrm{o}}|-| \mathrm{F}_{\mathrm{c}} \| / \Sigma\left|\mathrm{F}_{\mathrm{o}}\right|
\end{aligned}
$$

Goof $=\mathrm{S}=\left[\Sigma\left[\mathrm{w}\left(\mathrm{F}_{\mathrm{o}}{ }^{2}-\mathrm{F}_{\mathrm{c}}\right)^{2}\right] /(\mathrm{n}-\mathrm{p})\right]^{1 / 2}$ where $\mathrm{n}$ is the number of reflections and $\mathrm{p}$ is the total

number of parameters refined.

The thermal ellipsoid plot is shown at the $50 \%$ probability level. 
Table 1. Crystal data and structure refinement for S-26.

Identification code

Empirical formula

Formula weight

Temperature

Wavelength

Crystal system

Space group

Unit cell dimensions

Volume

Z

Density (calculated)

Absorption coefficient

$\mathrm{F}(000)$

Crystal color

Crystal size

Theta range for data collection

Index ranges

Reflections collected

Independent reflections

Completeness to theta $=28.32^{\circ}$

Absorption correction

Max. and min. transmission

Refinement method

Data / restraints / parameters

Goodness-of-fit on $\mathrm{F}^{2}$
S-26 (Glen Baghdasarian)

$\mathrm{C}_{15} \mathrm{H}_{21} \mathrm{~N} \mathrm{O}_{3}$

263.33

163(2) K

$0.71073 \AA$

Monoclinic

$P 2{ }_{1} / c$

$\mathrm{a}=8.8274(14) \AA \quad \alpha=90^{\circ}$.

$\mathrm{b}=42.094(7) \AA$

$\beta=104.349(3)^{\circ}$.

$\mathrm{c}=7.8988(13) \AA$

$\gamma=90^{\circ}$.

$2843.5(8) \AA^{3}$

8

$1.230 \mathrm{Mg} / \mathrm{m}^{3}$

$0.085 \mathrm{~mm}^{-1}$

1136

colorless

$0.43 \times 0.30 \times 0.25 \mathrm{~mm}^{3}$

1.94 to $28.32^{\circ}$.

$-11 \leq h \leq 11,-54 \leq k \leq 54,-10 \leq l \leq 10$

29952

$6853[\mathrm{R}(\mathrm{int})=0.0312]$

$96.5 \%$

Semi-empirical from equivalents

0.9790 and 0.9643

Full-matrix least-squares on $\mathrm{F}^{2}$

6853 / 0 / 511

1.085

Final $\mathrm{R}$ indices $[\mathrm{I}>2 \operatorname{sigma}(\mathrm{I})=5559$ data $]$

$\mathrm{R}$ indices (all data)

$\mathrm{R} 1=0.0421, \mathrm{wR} 2=0.0987$

Largest diff. peak and hole

$\mathrm{R} 1=0.0562, \mathrm{wR} 2=0.1082$

0.404 and -0.253 e. $\AA^{-3}$

Table 2. Atomic coordinates $\left(\mathrm{x} 10^{4}\right)$ and equivalent isotropic displacement parameters $\left(\AA^{2} \times 10^{3}\right)$

for $\mathbf{S - 2 6 . ~} U(\mathrm{eq})$ is defined as one third of the trace of the orthogonalized $\mathrm{U}^{\mathrm{ij}}$ tensor. 
$\begin{array}{lllll}\mathrm{x} & \mathrm{y} & \mathrm{z} & \mathrm{U}(\mathrm{eq})\end{array}$

\begin{tabular}{|c|c|c|c|c|}
\hline $\mathrm{O}(1)$ & $12176(1)$ & 1431(1) & $19579(1)$ & $23(1)$ \\
\hline $\mathrm{O}(2)$ & 15212(1) & $267(1)$ & 16878(1) & $30(1)$ \\
\hline $\mathrm{O}(3)$ & $11327(1)$ & $557(1)$ & $16161(1)$ & $16(1)$ \\
\hline $\mathrm{N}(1)$ & $13205(2)$ & $249(1)$ & $14473(2)$ & $26(1)$ \\
\hline$C(1)$ & $12976(1)$ & $603(1)$ & $16840(2)$ & $16(1)$ \\
\hline$C(2)$ & $13486(2)$ & $597(1)$ & $18838(2)$ & $17(1)$ \\
\hline$C(3)$ & 12679(2) & $858(1)$ & 19634(2) & $17(1)$ \\
\hline$C(4)$ & $13017(2)$ & 1184(1) & $18970(2)$ & $18(1)$ \\
\hline$C(5)$ & $12510(2)$ & 1194(1) & $16983(2)$ & 19(1) \\
\hline$C(6)$ & 13301(2) & $933(1)$ & $16174(2)$ & $18(1)$ \\
\hline$C(7)$ & $13894(2)$ & $354(1)$ & $16067(2)$ & 19(1) \\
\hline $\mathrm{C}(8)$ & $10715(2)$ & $284(1)$ & $16823(2)$ & 21(1) \\
\hline C(9) & $12822(2)$ & $1523(1)$ & $21337(2)$ & $23(1)$ \\
\hline$C(10)$ & $11855(2)$ & $1787(1)$ & $21817(2)$ & $21(1)$ \\
\hline $\mathrm{C}(11)$ & $10420(2)$ & 1873(1) & $20742(2)$ & $24(1)$ \\
\hline$C(12)$ & $9527(2)$ & $2110(1)$ & $21249(2)$ & $29(1)$ \\
\hline$C(13)$ & $10066(2)$ & $2264(1)$ & $22830(2)$ & $32(1)$ \\
\hline$C(14)$ & $11498(2)$ & 2181(1) & $23909(2)$ & $36(1)$ \\
\hline$C(15)$ & $12394(2)$ & 1944(1) & $23408(2)$ & $29(1)$ \\
\hline $\mathrm{O}(4)$ & 6912(1) & $1402(1)$ & $14649(1)$ & $20(1)$ \\
\hline $\mathrm{O}(5)$ & $10400(1)$ & $331(1)$ & 11606(1) & 19(1) \\
\hline $\mathrm{O}(6)$ & $7226(1)$ & $843(1)$ & 9852(1) & $16(1)$ \\
\hline $\mathrm{N}(2)$ & $8141(1)$ & $237(1)$ & $9583(2)$ & $18(1)$ \\
\hline$C(16)$ & $8296(1)$ & $714(1)$ & $11366(2)$ & $14(1)$ \\
\hline$C(17)$ & $7288(2)$ & $614(1)$ & $12612(2)$ & $16(1)$ \\
\hline$C(18)$ & $6549(2)$ & 901(1) & $13281(2)$ & $18(1)$ \\
\hline$C(19)$ & $7768(2)$ & $1146(1)$ & $14131(2)$ & $17(1)$ \\
\hline $\mathrm{C}(20)$ & $8742(2)$ & $1248(1)$ & $12871(2)$ & $17(1)$ \\
\hline$C(21)$ & $9516(1)$ & $956(1)$ & $12278(2)$ & $16(1)$ \\
\hline$C(22)$ & 9051(1) & $411(1)$ & $10842(2)$ & $15(1)$ \\
\hline$C(23)$ & $7956(2)$ & $965(1)$ & $8565(2)$ & $22(1)$ \\
\hline$C(24)$ & $7878(2)$ & $1644(1)$ & $15632(2)$ & $22(1)$ \\
\hline$C(25)$ & $6859(2)$ & 1861(1) & $16388(2)$ & $20(1)$ \\
\hline
\end{tabular}




$\begin{array}{lllll}\mathrm{C}(26) & 6881(2) & 2186(1) & 16119(2) & 27(1) \\ \mathrm{C}(27) & 5940(2) & 2389(1) & 16808(2) & 31(1) \\ \mathrm{C}(28) & 4950(2) & 2268(1) & 17751(2) & 28(1) \\ \mathrm{C}(29) & 4916(2) & 1942(1) & 18028(2) & 30(1) \\ \mathrm{C}(30) & 5876(2) & 1742(1) & 17365(2) & 27(1)\end{array}$

Table 3. Bond lengths $[\AA]$ and angles $\left[{ }^{\circ}\right]$ for S-26.

\begin{tabular}{ll}
\hline $\mathrm{O}(1)-\mathrm{C}(9)$ & $1.4179(17)$ \\
$\mathrm{O}(1)-\mathrm{C}(4)$ & $1.4303(15)$ \\
$\mathrm{O}(2)-\mathrm{C}(7)$ & $1.2364(16)$ \\
$\mathrm{O}(3)-\mathrm{C}(8)$ & $1.4247(16)$ \\
$\mathrm{O}(3)-\mathrm{C}(1)$ & $1.4346(15)$ \\
$\mathrm{N}(1)-\mathrm{C}(7)$ & $1.3314(17)$ \\
$\mathrm{C}(1)-\mathrm{C}(2)$ & $1.5304(17)$ \\
$\mathrm{C}(1)-\mathrm{C}(6)$ & $1.5341(18)$ \\
$\mathrm{C}(1)-\mathrm{C}(7)$ & $1.5409(17)$ \\
$\mathrm{C}(2)-\mathrm{C}(3)$ & $1.5276(17)$ \\
$\mathrm{C}(3)-\mathrm{C}(4)$ & $1.5241(18)$ \\
$\mathrm{C}(4)-\mathrm{C}(5)$ & $1.5220(18)$ \\
$\mathrm{C}(5)-\mathrm{C}(6)$ & $1.5272(18)$ \\
$\mathrm{C}(9)-\mathrm{C}(10)$ & $1.5052(19)$ \\
$\mathrm{C}(10)-\mathrm{C}(11)$ & $1.387(2)$ \\
$\mathrm{C}(10)-\mathrm{C}(15)$ & $1.395(2)$ \\
$\mathrm{C}(11)-\mathrm{C}(12)$ & $1.393(2)$ \\
$\mathrm{C}(12)-\mathrm{C}(13)$ & $1.383(2)$ \\
$\mathrm{C}(13)-\mathrm{C}(14)$ & $1.383(2)$ \\
$\mathrm{C}(14)-\mathrm{C}(15)$ & $1.391(2)$ \\
$\mathrm{O}(4)-\mathrm{C}(24)$ & $1.4263(16)$ \\
$\mathrm{O}(4)-\mathrm{C}(19)$ & $1.4333(15)$ \\
$\mathrm{O}(5)-\mathrm{C}(22)$ & $1.2410(15)$ \\
$\mathrm{O}(6)-\mathrm{C}(23)$ & $1.4274(15)$ \\
$\mathrm{O}(6)-\mathrm{C}(16)$ & $1.4339(14)$ \\
$\mathrm{N}(2)-\mathrm{C}(22)$ & $1.3317(17)$ \\
\end{tabular}




\begin{tabular}{|c|c|}
\hline$C(16)-C(21)$ & $1.5272(17)$ \\
\hline$C(16)-C(17)$ & $1.5393(17)$ \\
\hline$C(16)-C(22)$ & $1.5427(17)$ \\
\hline$C(17)-C(18)$ & $1.5285(17)$ \\
\hline C(18)-C(19) & $1.5200(18)$ \\
\hline$C(19)-C(20)$ & $1.5300(17)$ \\
\hline$C(20)-C(21)$ & $1.5337(17)$ \\
\hline$C(24)-C(25)$ & $1.5064(18)$ \\
\hline$C(25)-C(26)$ & $1.3858(19)$ \\
\hline$C(25)-C(30)$ & $1.3900(19)$ \\
\hline$C(26)-C(27)$ & $1.392(2)$ \\
\hline$C(27)-C(28)$ & $1.378(2)$ \\
\hline $\mathrm{C}(28)-\mathrm{C}(29)$ & $1.388(2)$ \\
\hline C(29)-C(30) & $1.387(2)$ \\
\hline $\mathrm{C}(9)-\mathrm{O}(1)-\mathrm{C}(4)$ & $114.33(10)$ \\
\hline $\mathrm{C}(8)-\mathrm{O}(3)-\mathrm{C}(1)$ & $114.52(10)$ \\
\hline $\mathrm{O}(3)-\mathrm{C}(1)-\mathrm{C}(2)$ & $113.22(10)$ \\
\hline $\mathrm{O}(3)-\mathrm{C}(1)-\mathrm{C}(6)$ & $104.69(10)$ \\
\hline$C(2)-C(1)-C(6)$ & 109.76(10) \\
\hline $\mathrm{O}(3)-\mathrm{C}(1)-\mathrm{C}(7)$ & $110.09(10)$ \\
\hline$C(2)-C(1)-C(7)$ & $110.28(10)$ \\
\hline$C(6)-C(1)-C(7)$ & $108.58(10)$ \\
\hline $\mathrm{C}(3)-\mathrm{C}(2)-\mathrm{C}(1)$ & $111.30(10)$ \\
\hline$C(4)-C(3)-C(2)$ & $110.79(10)$ \\
\hline $\mathrm{O}(1)-\mathrm{C}(4)-\mathrm{C}(5)$ & $106.30(10)$ \\
\hline $\mathrm{O}(1)-\mathrm{C}(4)-\mathrm{C}(3)$ & $112.18(10)$ \\
\hline$C(5)-C(4)-C(3)$ & $110.43(11)$ \\
\hline$C(4)-C(5)-C(6)$ & $111.20(11)$ \\
\hline$C(5)-C(6)-C(1)$ & $111.40(10)$ \\
\hline $\mathrm{O}(2)-\mathrm{C}(7)-\mathrm{N}(1)$ & $122.80(12)$ \\
\hline $\mathrm{O}(2)-\mathrm{C}(7)-\mathrm{C}(1)$ & $121.07(11)$ \\
\hline $\mathrm{N}(1)-\mathrm{C}(7)-\mathrm{C}(1)$ & $116.09(11)$ \\
\hline $\mathrm{O}(1)-\mathrm{C}(9)-\mathrm{C}(10)$ & $109.42(11)$ \\
\hline$C(11)-C(10)-C(15)$ & 118.97(13) \\
\hline $\mathrm{C}(11)-\mathrm{C}(10)-\mathrm{C}(9)$ & $121.77(12)$ \\
\hline $\mathrm{C}(15)-\mathrm{C}(10)-\mathrm{C}(9)$ & 119.23(13) \\
\hline
\end{tabular}




$\begin{array}{ll}\mathrm{C}(10)-\mathrm{C}(11)-\mathrm{C}(12) & 120.48(14) \\ \mathrm{C}(13)-\mathrm{C}(12)-\mathrm{C}(11) & 120.22(15) \\ \mathrm{C}(12)-\mathrm{C}(13)-\mathrm{C}(14) & 119.75(14) \\ \mathrm{C}(13)-\mathrm{C}(14)-\mathrm{C}(15) & 120.20(15) \\ \mathrm{C}(14)-\mathrm{C}(15)-\mathrm{C}(10) & 120.39(15) \\ \mathrm{C}(24)-\mathrm{O}(4)-\mathrm{C}(19) & 113.96(10) \\ \mathrm{C}(23)-\mathrm{O}(6)-\mathrm{C}(16) & 114.16(9) \\ \mathrm{O}(6)-\mathrm{C}(16)-\mathrm{C}(21) & 112.54(10) \\ \mathrm{O}(6)-\mathrm{C}(16)-\mathrm{C}(17) & 105.69(10) \\ \mathrm{C}(21)-\mathrm{C}(16)-\mathrm{C}(17) & 109.41(10) \\ \mathrm{O}(6)-\mathrm{C}(16)-\mathrm{C}(22) & 109.11(9) \\ \mathrm{C}(21)-\mathrm{C}(16)-\mathrm{C}(22) & 112.18(10) \\ \mathrm{C}(17)-\mathrm{C}(16)-\mathrm{C}(22) & 107.59(10) \\ \mathrm{C}(18)-\mathrm{C}(17)-\mathrm{C}(16) & 111.67(10) \\ \mathrm{C}(19)-\mathrm{C}(18)-\mathrm{C}(17) & 111.84(11) \\ \mathrm{O}(4)-\mathrm{C}(19)-\mathrm{C}(18) & 105.71(10) \\ \mathrm{O}(4)-\mathrm{C}(19)-\mathrm{C}(20) & 113.27(10) \\ \mathrm{C}(18)-\mathrm{C}(19)-\mathrm{C}(20) & 110.69(10) \\ \mathrm{C}(19)-\mathrm{C}(20)-\mathrm{C}(21) & 109.77(10) \\ \mathrm{C}(16)-\mathrm{C}(21)-\mathrm{C}(20) & 111.31(10) \\ \mathrm{O}(5)-\mathrm{C}(22)-\mathrm{N}(2) & 122.83(12) \\ \mathrm{O}(5)-\mathrm{C}(22)-\mathrm{C}(16) & 121.39(11) \\ \mathrm{N}(2)-\mathrm{C}(22)-\mathrm{C}(16) & 115.71(11) \\ \mathrm{O}(4)-\mathrm{C}(24)-\mathrm{C}(25) & 108.33(11) \\ \mathrm{C}(26)-\mathrm{C}(25)-\mathrm{C}(30) & 118.46(13) \\ \mathrm{C}(26)-\mathrm{C}(25)-\mathrm{C}(24) & 120.46(13) \\ \mathrm{C}(30)-\mathrm{C}(25)-\mathrm{C}(24) & 121.09(12) \\ \mathrm{C}(25)-\mathrm{C}(26)-\mathrm{C}(27) & 120.80(14) \\ \mathrm{C}(28)-\mathrm{C}(27)-\mathrm{C}(26) & 120.31(14) \\ \mathrm{C}(27)-\mathrm{C}(28)-\mathrm{C}(29) & 119.44(14) \\ \mathrm{C}(30)-\mathrm{C}(29)-\mathrm{C}(28) & 120.09(14) \\ \mathrm{C}(29)-\mathrm{C}(30)-\mathrm{C}(25) & 120.89(14) \\ & \\ & \end{array}$

Table 4. Anisotropic displacement parameters $\left(\AA^{2} \times 10^{3}\right)$ for S-26. The anisotropic displacement factor exponent takes the form: $-2 \pi^{2}\left[h^{2} a^{* 2} U^{11}+\ldots+2 h k a^{*} b^{*} U^{12}\right]$ 


\begin{tabular}{|c|c|c|c|c|c|c|}
\hline & $\mathrm{U}^{11}$ & $\mathrm{U}^{22}$ & $\mathrm{U}^{33}$ & $\mathrm{U}^{23}$ & $\mathrm{U}^{13}$ & $\mathrm{U}^{12}$ \\
\hline $\mathrm{O}(1)$ & $24(1)$ & $22(1)$ & $22(1)$ & $-7(1)$ & $4(1)$ & $6(1)$ \\
\hline $\mathrm{O}(2)$ & $20(1)$ & $39(1)$ & $26(1)$ & $-14(1)$ & $-5(1)$ & $14(1)$ \\
\hline $\mathrm{O}(3)$ & $12(1)$ & $18(1)$ & $18(1)$ & 1(1) & $2(1)$ & $1(1)$ \\
\hline $\mathrm{N}(1)$ & $18(1)$ & $35(1)$ & $20(1)$ & $-11(1)$ & $-2(1)$ & 11(1) \\
\hline $\mathrm{C}(1)$ & $12(1)$ & $19(1)$ & $16(1)$ & $-3(1)$ & $3(1)$ & $3(1)$ \\
\hline$C(2)$ & $15(1)$ & $17(1)$ & $16(1)$ & $-2(1)$ & $2(1)$ & $2(1)$ \\
\hline$C(3)$ & $17(1)$ & $19(1)$ & $16(1)$ & $-2(1)$ & $5(1)$ & $1(1)$ \\
\hline$C(4)$ & $17(1)$ & $17(1)$ & $20(1)$ & $-3(1)$ & $5(1)$ & $2(1)$ \\
\hline$C(5)$ & $20(1)$ & $17(1)$ & $20(1)$ & $1(1)$ & $5(1)$ & $1(1)$ \\
\hline$C(6)$ & $15(1)$ & $23(1)$ & $17(1)$ & $-2(1)$ & $6(1)$ & $-1(1)$ \\
\hline$C(7)$ & $16(1)$ & $23(1)$ & $19(1)$ & $-4(1)$ & $2(1)$ & $3(1)$ \\
\hline$C(8)$ & $21(1)$ & $20(1)$ & $21(1)$ & $0(1)$ & $4(1)$ & $-3(1)$ \\
\hline$C(9)$ & $23(1)$ & $23(1)$ & $22(1)$ & $-5(1)$ & $3(1)$ & $3(1)$ \\
\hline$C(10)$ & $25(1)$ & $17(1)$ & $22(1)$ & $-1(1)$ & $10(1)$ & $-2(1)$ \\
\hline$C(11)$ & $29(1)$ & $22(1)$ & $22(1)$ & $-3(1)$ & $7(1)$ & $2(1)$ \\
\hline$C(12)$ & $30(1)$ & $26(1)$ & $30(1)$ & $1(1)$ & $8(1)$ & $7(1)$ \\
\hline$C(13)$ & $37(1)$ & $29(1)$ & $34(1)$ & $-5(1)$ & $16(1)$ & $8(1)$ \\
\hline$C(14)$ & $40(1)$ & $38(1)$ & $29(1)$ & $-15(1)$ & $8(1)$ & $4(1)$ \\
\hline$C(15)$ & $28(1)$ & $32(1)$ & $27(1)$ & $-9(1)$ & $4(1)$ & $2(1)$ \\
\hline $\mathrm{O}(4)$ & $19(1)$ & $20(1)$ & $24(1)$ & $-8(1)$ & $7(1)$ & $1(1)$ \\
\hline $\mathrm{O}(5)$ & $16(1)$ & $21(1)$ & $19(1)$ & $-2(1)$ & $1(1)$ & $5(1)$ \\
\hline $\mathrm{O}(6)$ & $14(1)$ & $20(1)$ & $14(1)$ & $2(1)$ & $3(1)$ & $3(1)$ \\
\hline $\mathrm{N}(2)$ & $16(1)$ & $19(1)$ & $19(1)$ & $-4(1)$ & $1(1)$ & $4(1)$ \\
\hline$C(16)$ & $13(1)$ & $16(1)$ & $13(1)$ & $1(1)$ & $3(1)$ & $2(1)$ \\
\hline$C(17)$ & $18(1)$ & $16(1)$ & $16(1)$ & $0(1)$ & $6(1)$ & $-1(1)$ \\
\hline$C(18)$ & $17(1)$ & $21(1)$ & $19(1)$ & $-2(1)$ & $8(1)$ & $-1(1)$ \\
\hline$C(19)$ & $18(1)$ & $17(1)$ & $16(1)$ & $-2(1)$ & $6(1)$ & $2(1)$ \\
\hline$C(20)$ & $17(1)$ & $18(1)$ & $18(1)$ & $-2(1)$ & $5(1)$ & $-1(1)$ \\
\hline$C(21)$ & $13(1)$ & $19(1)$ & $15(1)$ & $-1(1)$ & $4(1)$ & $0(1)$ \\
\hline$C(22)$ & $15(1)$ & $17(1)$ & $13(1)$ & $2(1)$ & $5(1)$ & $1(1)$ \\
\hline $\mathrm{C}(23)$ & $21(1)$ & $28(1)$ & $17(1)$ & $7(1)$ & $5(1)$ & $3(1)$ \\
\hline
\end{tabular}




$\begin{array}{lllllll}\mathrm{C}(24) & 23(1) & 19(1) & 27(1) & -5(1) & 11(1) & -3(1) \\ \mathrm{C}(25) & 21(1) & 19(1) & 19(1) & -3(1) & 4(1) & -1(1) \\ \mathrm{C}(26) & 36(1) & 21(1) & 25(1) & -1(1) & 12(1) & -3(1) \\ \mathrm{C}(27) & 44(1) & 17(1) & 31(1) & -1(1) & 9(1) & 3(1) \\ \mathrm{C}(28) & 32(1) & 26(1) & 27(1) & -9(1) & 7(1) & 5(1) \\ \mathrm{C}(29) & 32(1) & 28(1) & 34(1) & -5(1) & 18(1) & -1(1) \\ \mathrm{C}(30) & 32(1) & 18(1) & 35(1) & -1(1) & 16(1) & -1(1)\end{array}$

Table 5. Hydrogen coordinates ( $\mathrm{x} 10^{4}$ ) and isotropic displacement parameters $\left(\AA^{2} \times 10^{3}\right)$ for $\mathbf{S}-26$.

$\begin{array}{llll}\mathrm{x} & \mathrm{z} & \mathrm{U}(\mathrm{eq})\end{array}$

$\begin{array}{lrrrr}\mathrm{H}(1 \mathrm{~A}) & 12310(20) & 316(4) & 13950(20) & 34(5) \\ \mathrm{H}(1 \mathrm{~B}) & 13700(20) & 103(5) & 13970(20) & 35(5) \\ \mathrm{H}(2 \mathrm{~A}) & 14608(19) & 632(4) & 19180(20) & 20(4) \\ \mathrm{H}(2 \mathrm{~B}) & 13279(18) & 382(4) & 19290(20) & 18(4) \\ \mathrm{H}(3 \mathrm{~A}) & 11522(19) & 830(4) & 19320(20) & 20(4) \\ \mathrm{H}(3 \mathrm{~B}) & 13021(19) & 847(4) & 20920(20) & 21(4) \\ \mathrm{H}(4) & 14165(18) & 1225(3) & 19382(19) & 14(3) \\ \mathrm{H}(5 \mathrm{~A}) & 11367(19) & 1173(4) & 16630(20) & 20(4) \\ \mathrm{H}(5 \mathrm{~B}) & 12765(19) & 1407(4) & 16560(20) & 23(4) \\ \mathrm{H}(6 \mathrm{~A}) & 14419(19) & 968(4) & 16470(20) & 17(4) \\ \mathrm{H}(6 \mathrm{~B}) & 12952(19) & 941(4) & 14880(20) & 23(4) \\ \mathrm{H}(8 \mathrm{~A}) & 11310(20) & 90(4) & 16660(20) & 27(4) \\ \mathrm{H}(8 \mathrm{~B}) & 9640(20) & 263(4) & 16120(20) & 36(5) \\ \mathrm{H}(8 \mathrm{C}) & 10760(20) & 307(4) & 18100(20) & 26(4) \\ \mathrm{H}(9 \mathrm{~A}) & 13930(20) & 1590(4) & 21500(20) & 29(4) \\ \mathrm{H}(9 B) & 12880(20) & 1343(4) & 22180(20) & 28(4) \\ \mathrm{H}(11) & 10040(20) & 1769(4) & 19610(20) & 33(5) \\ \mathrm{H}(12) & 8560(20) & 2166(4) & 20490(20) & 34(5)\end{array}$




$\begin{array}{lrrrr}\mathrm{H}(13) & 9460(20) & 2432(5) & 23170(30) & 41(5) \\ \mathrm{H}(14) & 11850(20) & 2295(5) & 25020(30) & 46(5) \\ \mathrm{H}(15) & 13400(20) & 1882(4) & 24180(20) & 35(5) \\ \mathrm{H}(2 \mathrm{C}) & 7190(20) & 307(4) & 9000(20) & 33(5) \\ \mathrm{H}(2 \mathrm{D}) & 8510(20) & 55(5) & 9250(20) & 37(5) \\ \mathrm{H}(17 \mathrm{~A}) & 7995(18) & 496(4) & 13620(20) & 21(4) \\ \mathrm{H}(17 \mathrm{~B}) & 6470(19) & 463(4) & 12030(20) & 23(4) \\ \mathrm{H}(18 \mathrm{~A}) & 5975(19) & 826(4) & 14100(20) & 24(4) \\ \mathrm{H}(18 \mathrm{~B}) & 5795(19) & 1009(4) & 12290(20) & 21(4) \\ \mathrm{H}(19) & 8488(17) & 1055(3) & 15229(19) & 14(3) \\ \mathrm{H}(20 \mathrm{~A}) & 9552(19) & 1395(4) & 13450(20) & 19(4) \\ \mathrm{H}(20 \mathrm{~B}) & 8050(19) & 1355(4) & 11850(20) & 23(4) \\ \mathrm{H}(21 \mathrm{~A}) & 10172(18) & 1022(4) & 11520(20) & 20(4) \\ \mathrm{H}(21 \mathrm{~B}) & 10180(18) & 848(4) & 13290(20) & 20(4) \\ \mathrm{H}(23 \mathrm{~A}) & 8670(20) & 809(4) & 8270(20) & 30(4) \\ \mathrm{H}(23 \mathrm{~B}) & 7110(20) & 1011(4) & 7530(20) & 30(4) \\ \mathrm{H}(23 \mathrm{C}) & 8520(20) & 1166(4) & 8960(20) & 30(4) \\ \mathrm{H}(24 \mathrm{~A}) & 8700(20) & 1541(4) & 16590(20) & 28(4) \\ \mathrm{H}(24 \mathrm{~B}) & 8400(20) & 1768(4) & 14890(20) & 28(4) \\ \mathrm{H}(26) & 7560(20) & 2270(5) & 15410(20) & 39(5) \\ \mathrm{H}(27) & 5960(20) & 2612(5) & 16590(30) & 42(5) \\ \mathrm{H}(28) & 4280(20) & 2403(4) & 18230(20) & 35(5) \\ \mathrm{H}(29) & 4210(20) & 1859(5) & 18670(30) & 42(5) \\ \mathrm{H}(30) & 5820(20) & 1513(4) & 17570(20) & 34(5)\end{array}$

Table 6. Torsion angles $\left[^{\circ}\right]$ for $\mathbf{S - 2 6}$.

\begin{tabular}{lc}
\hline $\mathrm{C}(8)-\mathrm{O}(3)-\mathrm{C}(1)-\mathrm{C}(2)$ & $-51.91(14)$ \\
$\mathrm{C}(8)-\mathrm{O}(3)-\mathrm{C}(1)-\mathrm{C}(6)$ & $-171.44(10)$ \\
$\mathrm{C}(8)-\mathrm{O}(3)-\mathrm{C}(1)-\mathrm{C}(7)$ & $72.04(13)$
\end{tabular}




\begin{tabular}{|c|c|}
\hline $\mathrm{O}(3)-\mathrm{C}(1)-\mathrm{C}(2)-\mathrm{C}(3)$ & $-60.25(14)$ \\
\hline$C(6)-C(1)-C(2)-C(3)$ & $56.33(13)$ \\
\hline$C(7)-C(1)-C(2)-C(3)$ & $175.91(10)$ \\
\hline$C(1)-C(2)-C(3)-C(4)$ & $-57.49(14)$ \\
\hline $\mathrm{C}(9)-\mathrm{O}(1)-\mathrm{C}(4)-\mathrm{C}(5)$ & $-161.59(11)$ \\
\hline $\mathrm{C}(9)-\mathrm{O}(1)-\mathrm{C}(4)-\mathrm{C}(3)$ & $77.61(14)$ \\
\hline $\mathrm{C}(2)-\mathrm{C}(3)-\mathrm{C}(4)-\mathrm{O}(1)$ & $175.25(10)$ \\
\hline$C(2)-C(3)-C(4)-C(5)$ & $56.87(14)$ \\
\hline $\mathrm{O}(1)-\mathrm{C}(4)-\mathrm{C}(5)-\mathrm{C}(6)$ & $-178.36(10)$ \\
\hline$C(3)-C(4)-C(5)-C(6)$ & $-56.45(14)$ \\
\hline$C(4)-C(5)-C(6)-C(1)$ & $56.42(14)$ \\
\hline $\mathrm{O}(3)-\mathrm{C}(1)-\mathrm{C}(6)-\mathrm{C}(5)$ & $66.10(13)$ \\
\hline$C(2)-C(1)-C(6)-C(5)$ & $-55.72(13)$ \\
\hline$C(7)-C(1)-C(6)-C(5)$ & $-176.34(11)$ \\
\hline $\mathrm{O}(3)-\mathrm{C}(1)-\mathrm{C}(7)-\mathrm{O}(2)$ & $-152.54(13)$ \\
\hline $\mathrm{C}(2)-\mathrm{C}(1)-\mathrm{C}(7)-\mathrm{O}(2)$ & $-26.91(18)$ \\
\hline $\mathrm{C}(6)-\mathrm{C}(1)-\mathrm{C}(7)-\mathrm{O}(2)$ & $93.39(15)$ \\
\hline $\mathrm{O}(3)-\mathrm{C}(1)-\mathrm{C}(7)-\mathrm{N}(1)$ & $29.64(16)$ \\
\hline $\mathrm{C}(2)-\mathrm{C}(1)-\mathrm{C}(7)-\mathrm{N}(1)$ & $155.28(12)$ \\
\hline $\mathrm{C}(6)-\mathrm{C}(1)-\mathrm{C}(7)-\mathrm{N}(1)$ & $-84.43(15)$ \\
\hline $\mathrm{C}(4)-\mathrm{O}(1)-\mathrm{C}(9)-\mathrm{C}(10)$ & $179.15(11)$ \\
\hline $\mathrm{O}(1)-\mathrm{C}(9)-\mathrm{C}(10)-\mathrm{C}(11)$ & $10.42(19)$ \\
\hline $\mathrm{O}(1)-\mathrm{C}(9)-\mathrm{C}(10)-\mathrm{C}(15)$ & $-171.45(13)$ \\
\hline$C(15)-C(10)-C(11)-C(12)$ & $-0.4(2)$ \\
\hline$C(9)-C(10)-C(11)-C(12)$ & $177.71(14)$ \\
\hline$C(10)-C(11)-C(12)-C(13)$ & $0.3(2)$ \\
\hline$C(11)-C(12)-C(13)-C(14)$ & $-0.1(3)$ \\
\hline$C(12)-C(13)-C(14)-C(15)$ & $0.0(3)$ \\
\hline$C(13)-C(14)-C(15)-C(10)$ & $-0.2(3)$ \\
\hline$C(11)-C(10)-C(15)-C(14)$ & $0.4(2)$ \\
\hline$C(9)-C(10)-C(15)-C(14)$ & $-177.80(15)$ \\
\hline $\mathrm{C}(23)-\mathrm{O}(6)-\mathrm{C}(16)-\mathrm{C}(21)$ & $57.60(13)$ \\
\hline $\mathrm{C}(23)-\mathrm{O}(6)-\mathrm{C}(16)-\mathrm{C}(17)$ & $176.96(10)$ \\
\hline $\mathrm{C}(23)-\mathrm{O}(6)-\mathrm{C}(16)-\mathrm{C}(22)$ & $-67.60(13)$ \\
\hline $\mathrm{O}(6)-\mathrm{C}(16)-\mathrm{C}(17)-\mathrm{C}(18)$ & $-66.33(13)$ \\
\hline$C(21)-C(16)-C(17)-C(18)$ & $55.07(14)$ \\
\hline
\end{tabular}




\begin{tabular}{lc}
$\mathrm{C}(22)-\mathrm{C}(16)-\mathrm{C}(17)-\mathrm{C}(18)$ & $177.19(10)$ \\
$\mathrm{C}(16)-\mathrm{C}(17)-\mathrm{C}(18)-\mathrm{C}(19)$ & $-54.76(14)$ \\
$\mathrm{C}(24)-\mathrm{O}(4)-\mathrm{C}(19)-\mathrm{C}(18)$ & $174.21(11)$ \\
$\mathrm{C}(24)-\mathrm{O}(4)-\mathrm{C}(19)-\mathrm{C}(20)$ & $-64.42(14)$ \\
$\mathrm{C}(17)-\mathrm{C}(18)-\mathrm{C}(19)-\mathrm{O}(4)$ & $178.54(10)$ \\
$\mathrm{C}(17)-\mathrm{C}(18)-\mathrm{C}(19)-\mathrm{C}(20)$ & $55.52(14)$ \\
$\mathrm{O}(4)-\mathrm{C}(19)-\mathrm{C}(20)-\mathrm{C}(21)$ & $-175.71(10)$ \\
$\mathrm{C}(18)-\mathrm{C}(19)-\mathrm{C}(20)-\mathrm{C}(21)$ & $-57.19(14)$ \\
$\mathrm{O}(6)-\mathrm{C}(16)-\mathrm{C}(21)-\mathrm{C}(20)$ & $59.42(13)$ \\
$\mathrm{C}(17)-\mathrm{C}(16)-\mathrm{C}(21)-\mathrm{C}(20)$ & $-57.76(13)$ \\
$\mathrm{C}(22)-\mathrm{C}(16)-\mathrm{C}(21)-\mathrm{C}(20)$ & $-177.08(10)$ \\
$\mathrm{C}(19)-\mathrm{C}(20)-\mathrm{C}(21)-\mathrm{C}(16)$ & $59.27(14)$ \\
$\mathrm{O}(6)-\mathrm{C}(16)-\mathrm{C}(22)-\mathrm{O}(5)$ & $149.10(11)$ \\
$\mathrm{C}(21)-\mathrm{C}(16)-\mathrm{C}(22)-\mathrm{O}(5)$ & $23.70(16)$ \\
$\mathrm{C}(17)-\mathrm{C}(16)-\mathrm{C}(22)-\mathrm{O}(5)$ & $-96.68(13)$ \\
$\mathrm{O}(6)-\mathrm{C}(16)-\mathrm{C}(22)-\mathrm{N}(2)$ & $-33.64(14)$ \\
$\mathrm{C}(21)-\mathrm{C}(16)-\mathrm{C}(22)-\mathrm{N}(2)$ & $-159.05(11)$ \\
$\mathrm{C}(17)-\mathrm{C}(16)-\mathrm{C}(22)-\mathrm{N}(2)$ & $80.57(13)$ \\
$\mathrm{C}(19)-\mathrm{O}(4)-\mathrm{C}(24)-\mathrm{C}(25)$ & $-168.91(11)$ \\
$\mathrm{O}(4)-\mathrm{C}(24)-\mathrm{C}(25)-\mathrm{C}(26)$ & $-126.80(14)$ \\
$\mathrm{O}(4)-\mathrm{C}(24)-\mathrm{C}(25)-\mathrm{C}(30)$ & $53.10(18)$ \\
$\mathrm{C}(30)-\mathrm{C}(25)-\mathrm{C}(26)-\mathrm{C}(27)$ & $-0.1(2)$ \\
$\mathrm{C}(24)-\mathrm{C}(25)-\mathrm{C}(26)-\mathrm{C}(27)$ & $179.79(14)$ \\
$\mathrm{C}(25)-\mathrm{C}(26)-\mathrm{C}(27)-\mathrm{C}(28)$ & $-0.9(2)$ \\
$\mathrm{C}(26)-\mathrm{C}(27)-\mathrm{C}(28)-\mathrm{C}(29)$ & $0.8(2)$ \\
$\mathrm{C}(27)-\mathrm{C}(28)-\mathrm{C}(29)-\mathrm{C}(30)$ & $0.3(2)$ \\
$\mathrm{C}(28)-\mathrm{C}(29)-\mathrm{C}(30)-\mathrm{C}(25)$ & $-1.4(2)$ \\
$\mathrm{C}(26)-\mathrm{C}(25)-\mathrm{C}(30)-\mathrm{C}(29)$ & $1.3(2)$ \\
$\mathrm{C}(24)-\mathrm{C}(25)-\mathrm{C}(30)-\mathrm{C}(29)$ & $-178.64(14)$ \\
\hline &
\end{tabular}

\section{Amide S-27}




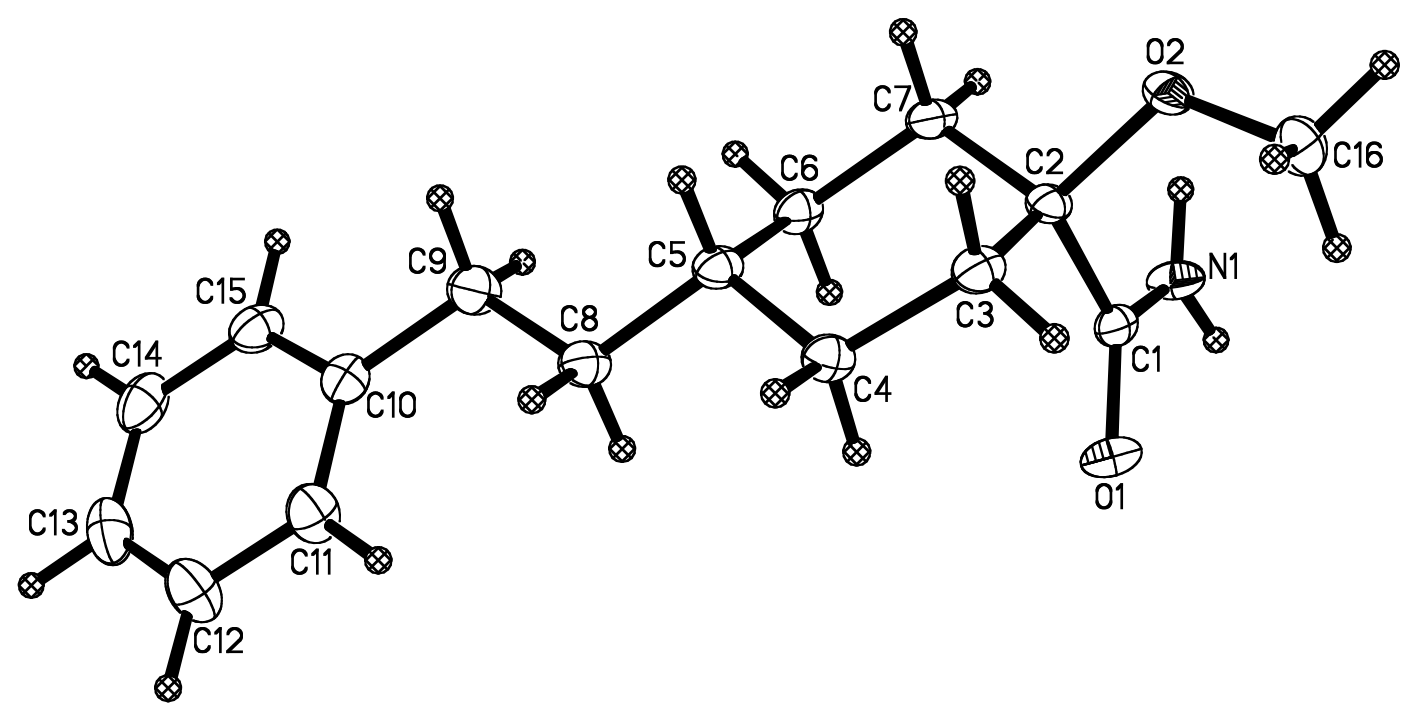

X-ray Data Collection, Structure Solution and Refinement for S-27.

A colorless crystal of approximate dimensions $0.08 \times 0.38 \times 0.44 \mathrm{~mm}$ was mounted on a glass fiber and transferred to a Bruker CCD platform diffractometer. The SMART ${ }^{1}$ program package was used to determine the unit-cell parameters and for data collection $(25 \mathrm{sec} /$ frame scan time for a sphere of diffraction data). The raw frame data was processed using SAINT ${ }^{2}$ and $\mathrm{SADABS}^{3}$ to yield the reflection data file. Subsequent calculations were carried out using the SHELXTL ${ }^{4}$ program. The diffraction symmetry was $2 / m$ and the systematic absences were consistent with the centrosymmetric monoclinic space group $P 2_{1} / n$ which was later determined to be correct.

The structure was solved by direct methods and refined on $\mathrm{F}^{2}$ by full-matrix least-squares techniques. The analytical scattering factors ${ }^{5}$ for neutral atoms were used throughout the analysis. Hydrogen atoms were located from a difference-Fourier map and refined (x,y,z and $\mathrm{U}_{\text {iso }}$ ). At convergence, $\mathrm{wR} 2=0.1097$ and Goof $=1.054$ for 265 variables refined against 2909 data. As a comparison for refinement on F, R1 $=0.0412$ for those 2309 data with $\mathrm{I}>2.0 \sigma(\mathrm{I})$.

Definitions:

$$
\begin{aligned}
& \mathrm{wR} 2=\left[\Sigma\left[\mathrm{w}\left(\mathrm{F}_{\mathrm{o}}{ }^{2}-\mathrm{F}_{\mathrm{c}}{ }^{2}\right)^{2}\right] / \Sigma\left[\mathrm{w}\left(\mathrm{F}_{\mathrm{o}}{ }^{2}\right)^{2}\right]\right]^{1 / 2} \\
& \mathrm{R} 1=\Sigma|| \mathrm{F}_{\mathrm{o}}|-| \mathrm{F}_{\mathrm{c}} \| / \Sigma\left|\mathrm{F}_{\mathrm{o}}\right|
\end{aligned}
$$

Goof $=\mathrm{S}=\left[\Sigma\left[\mathrm{w}\left(\mathrm{F}_{\mathrm{o}}^{2}-\mathrm{F}_{\mathrm{c}}^{2}\right)^{2}\right] /(\mathrm{n}-\mathrm{p})\right]^{1 / 2}$ where $\mathrm{n}$ is the number of reflections and $\mathrm{p}$ is the total number of parameters refined.

The thermal ellipsoid plot is shown at the $50 \%$ probability level. 
Table 1. Crystal data and structure refinement for S-27.

Identification code

Empirical formula

Formula weight

Temperature

Wavelength

Crystal system

Space group

Unit cell dimensions

Volume

$\mathrm{Z}$

Density (calculated)

Absorption coefficient

$\mathrm{F}(000)$

Crystal color

Crystal size

Theta range for data collection

Index ranges

Reflections collected

Independent reflections

Completeness to theta $=26.37^{\circ}$

Absorption correction

Max. and min. transmission

Refinement method

Data / restraints / parameters

Goodness-of-fit on $\mathrm{F}^{2}$

Final $\mathrm{R}$ indices $[\mathrm{I}>2 \operatorname{sigma}(\mathrm{I})=2309$ data $]$

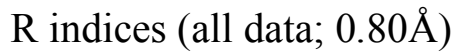

Extinction coefficient

Largest diff. peak and hole
S-27 (Glen Baghdasarian)

$\mathrm{C}_{16} \mathrm{H}_{23} \mathrm{~N} \mathrm{O}_{2}$

261.35

173(2) K

$0.71073 \AA$

Monoclinic

$P 2{ }_{1} / n$

$\mathrm{a}=6.6669(9) \AA \quad \alpha=90^{\circ}$.

$\mathrm{b}=32.636(5) \AA$

$\beta=104.551(2)^{\circ}$.

$\mathrm{c}=6.7762(10) \AA \quad \gamma=90^{\circ}$.

4

$1.216 \mathrm{Mg} / \mathrm{m}^{3}$

$0.079 \mathrm{~mm}^{-1}$

568

colorless

$0.44 \times 0.38 \times 0.08 \mathrm{~mm}^{3}$

2.50 to $26.37^{\circ}$.

$-8 \leq h \leq 8,-40 \leq k \leq 40,-8 \leq l \leq 8$

13270

$2909[\mathrm{R}(\mathrm{int})=0.0300]$

$99.2 \%$

Semi-empirical from equivalents

0.9937 and 0.9659

Full-matrix least-squares on $\mathrm{F}^{2}$

2909 / 0 / 265

1.054

$\mathrm{R} 1=0.0412, \mathrm{wR} 2=0.1007$

$\mathrm{R} 1=0.0551, \mathrm{wR} 2=0.1097$

$0.0028(16)$

0.322 and -0.166 e. $\AA^{-3}$

Table 2. Atomic coordinates ( $\mathrm{x} 10^{4}$ ) and equivalent isotropic displacement parameters 
$\left(\AA^{2} \times 10^{3}\right)$

for S-27. $U(e q)$ is defined as one third of the trace of the orthogonalized $U^{i j}$ tensor.

\begin{tabular}{lrrrr}
\hline & & & \\
& & & \\
& & & \\
& & & \\
\\
$(1)$ & & & & \\
$\mathrm{C}(2)$ & $7066(2)$ & $377(1)$ & $-3010(2)$ & $17(1)$ \\
$\mathrm{C}(3)$ & $8543(2)$ & $651(1)$ & $-1406(2)$ & $17(1)$ \\
$\mathrm{C}(4)$ & $7775(2)$ & $1093(1)$ & $-1495(2)$ & $20(1)$ \\
$\mathrm{C}(5)$ & $8046(2)$ & $1325(1)$ & $-3375(2)$ & $20(1)$ \\
$\mathrm{C}(6)$ & $10240(2)$ & $1301(1)$ & $-3674(2)$ & $19(1)$ \\
$\mathrm{C}(7)$ & $10905(2)$ & $853(1)$ & $-3660(2)$ & $20(1)$ \\
$\mathrm{C}(8)$ & $10748(2)$ & $640(1)$ & $-1692(2)$ & $20(1)$ \\
$\mathrm{C}(9)$ & $10314(2)$ & $1531(1)$ & $-5620(2)$ & $21(1)$ \\
$\mathrm{C}(10)$ & $12426(2)$ & $1541(1)$ & $-6104(2)$ & $25(1)$ \\
$\mathrm{C}(11)$ & $12531(2)$ & $1789(1)$ & $-7970(2)$ & $21(1)$ \\
$\mathrm{C}(12)$ & $10905(3)$ & $2033(1)$ & $-9028(2)$ & $27(1)$ \\
$\mathrm{C}(13)$ & $11058(3)$ & $2261(1)$ & $-10720(3)$ & $33(1)$ \\
$\mathrm{C}(14)$ & $12860(3)$ & $2249(1)$ & $-11377(3)$ & $33(1)$ \\
$\mathrm{C}(15)$ & $14489(3)$ & $2006(1)$ & $-10355(2)$ & $31(1)$ \\
$\mathrm{C}(16)$ & $14330(2)$ & $1779(1)$ & $-8675(2)$ & $26(1)$ \\
$\mathrm{N}(1)$ & $6838(3)$ & $469(1)$ & $1243(2)$ & $26(1)$ \\
$\mathrm{O}(1)$ & $7567(2)$ & $-17(1)$ & $-3020(2)$ & $23(1)$ \\
$\mathrm{O}(2)$ & $5511(2)$ & $523(1)$ & $-4194(2)$ & $23(1)$ \\
& $8712(2)$ & $470(1)$ & $579(1)$ & $21(1)$ \\
\hline & & & & \\
\hline
\end{tabular}

Table 3. Bond lengths $[\AA]$ and angles $\left[^{\circ}\right]$ for $\mathbf{S - 2 7}$.

\begin{tabular}{ll}
\hline $\mathrm{C}(1)-\mathrm{O}(1)$ & $1.2350(17)$ \\
$\mathrm{C}(1)-\mathrm{N}(1)$ & $1.329(2)$ \\
$\mathrm{C}(1)-\mathrm{C}(2)$ & $1.5533(19)$ \\
$\mathrm{C}(2)-\mathrm{O}(2)$ & $1.4475(16)$ \\
$\mathrm{C}(2)-\mathrm{C}(3)$ & $1.526(2)$
\end{tabular}




$\begin{array}{lc}\mathrm{C}(2)-\mathrm{C}(7) & 1.5306(19) \\ \mathrm{C}(3)-\mathrm{C}(4) & 1.532(2) \\ \mathrm{C}(4)-\mathrm{C}(5) & 1.5286(19) \\ \mathrm{C}(5)-\mathrm{C}(6) & 1.527(2) \\ \mathrm{C}(5)-\mathrm{C}(8) & 1.529(2) \\ \mathrm{C}(6)-\mathrm{C}(7) & 1.530(2) \\ \mathrm{C}(8)-\mathrm{C}(9) & 1.524(2) \\ \mathrm{C}(9)-\mathrm{C}(10) & 1.518(2) \\ \mathrm{C}(10)-\mathrm{C}(11) & 1.390(2) \\ \mathrm{C}(10)-\mathrm{C}(15) & 1.398(2) \\ \mathrm{C}(11)-\mathrm{C}(12) & 1.393(2) \\ \mathrm{C}(12)-\mathrm{C}(13) & 1.383(2) \\ \mathrm{C}(13)-\mathrm{C}(14) & 1.381(3) \\ \mathrm{C}(14)-\mathrm{C}(15) & 1.385(2) \\ \mathrm{C}(16)-\mathrm{O}(2) & 1.4301(18) \\ \mathrm{O}(1)-\mathrm{C}(1)-\mathrm{N}(1) & 122.65(13) \\ \mathrm{O}(1)-\mathrm{C}(1)-\mathrm{C}(2) & 120.98(13) \\ \mathrm{N}(1)-\mathrm{C}(1)-\mathrm{C}(2) & 116.38(12) \\ \mathrm{O}(2)-\mathrm{C}(2)-\mathrm{C}(3) & 111.70(11) \\ \mathrm{O}(2)-\mathrm{C}(2)-\mathrm{C}(7) & 104.79(11) \\ \mathrm{C}(3)-\mathrm{C}(2)-\mathrm{C}(7) & 109.86(12) \\ \mathrm{O}(2)-\mathrm{C}(2)-\mathrm{C}(1) & 107.64(11) \\ \mathrm{C}(3)-\mathrm{C}(2)-\mathrm{C}(1) & 111.62(11) \\ \mathrm{C}(7)-\mathrm{C}(2)-\mathrm{C}(1) & 111.01(11) \\ \mathrm{C}(2)-\mathrm{C}(3)-\mathrm{C}(4) & 113.08(11) \\ \mathrm{C}(5)-\mathrm{C}(4)-\mathrm{C}(3) & 113.67(12) \\ \mathrm{C}(6)-\mathrm{C}(5)-\mathrm{C}(4) & 109.55(12) \\ \mathrm{C}(6)-\mathrm{C}(5)-\mathrm{C}(8) & 113.81(12) \\ \mathrm{C}(4)-\mathrm{C}(5)-\mathrm{C}(8) & 109.16(12) \\ \mathrm{C}(5)-\mathrm{C}(6)-\mathrm{C}(7) & 110.85(12) \\ \mathrm{C}(6)-\mathrm{C}(7)-\mathrm{C}(2) & 112.23(12) \\ \mathrm{C}(9)-\mathrm{C}(8)-\mathrm{C}(5) & 115.28(12) \\ \mathrm{C}(10)-\mathrm{C}(9)-\mathrm{C}(8) & 115.37(13) \\ \mathrm{C}(11)-\mathrm{C}(10)-\mathrm{C}(15) & 117.51(14) \\ \mathrm{C}(11)-\mathrm{C}(10)-\mathrm{C}(9) & 122.80(13) \\ \mathrm{C}(15)-\mathrm{C}(10)-\mathrm{C}(9) & 119.69(14)\end{array}$




$\begin{array}{ll}\mathrm{C}(10)-\mathrm{C}(11)-\mathrm{C}(12) & 121.49(15) \\ \mathrm{C}(13)-\mathrm{C}(12)-\mathrm{C}(11) & 119.88(16) \\ \mathrm{C}(14)-\mathrm{C}(13)-\mathrm{C}(12) & 119.54(15) \\ \mathrm{C}(13)-\mathrm{C}(14)-\mathrm{C}(15) & 120.40(15) \\ \mathrm{C}(14)-\mathrm{C}(15)-\mathrm{C}(10) & 121.17(16) \\ \mathrm{C}(16)-\mathrm{O}(2)-\mathrm{C}(2) & 115.03(11)\end{array}$

Table 4. Anisotropic displacement parameters $\left(\AA^{2} \times 10^{3}\right)$ for S-27. The anisotropic displacement factor exponent takes the form: $-2 \pi^{2}\left[h^{2} a^{* 2} U^{11}+\ldots+2 h k a^{*} b^{*} U^{12}\right]$

\begin{tabular}{|c|c|c|c|c|c|c|}
\hline & $\mathrm{U}^{11}$ & $\mathrm{U}^{22}$ & $\mathrm{U}^{33}$ & $\mathrm{U}^{23}$ & $\mathrm{U}^{13}$ & $\mathrm{U}^{12}$ \\
\hline $\mathrm{C}(1)$ & $13(1)$ & $26(1)$ & $13(1)$ & $-1(1)$ & $5(1)$ & $-1(1)$ \\
\hline$C(2)$ & $15(1)$ & $25(1)$ & 11(1) & 1(1) & $3(1)$ & $0(1)$ \\
\hline$C(3)$ & $17(1)$ & $26(1)$ & $15(1)$ & $-3(1)$ & $5(1)$ & $0(1)$ \\
\hline $\mathrm{C}(4)$ & $18(1)$ & $23(1)$ & $19(1)$ & $0(1)$ & $5(1)$ & $3(1)$ \\
\hline$C(5)$ & $16(1)$ & $26(1)$ & $14(1)$ & $-1(1)$ & $4(1)$ & $0(1)$ \\
\hline$C(6)$ & $15(1)$ & $27(1)$ & $19(1)$ & $-1(1)$ & $6(1)$ & 1(1) \\
\hline$C(7)$ & $15(1)$ & $25(1)$ & $18(1)$ & $-1(1)$ & $1(1)$ & 1(1) \\
\hline $\mathrm{C}(8)$ & $19(1)$ & $28(1)$ & $18(1)$ & 1(1) & $5(1)$ & 2(1) \\
\hline$C(9)$ & $20(1)$ & $35(1)$ & $22(1)$ & $4(1)$ & $8(1)$ & $3(1)$ \\
\hline$C(10)$ & $22(1)$ & $24(1)$ & $20(1)$ & $-4(1)$ & $7(1)$ & $-3(1)$ \\
\hline $\mathrm{C}(11)$ & $28(1)$ & $27(1)$ & $30(1)$ & $3(1)$ & $14(1)$ & $4(1)$ \\
\hline$C(12)$ & $40(1)$ & $26(1)$ & $35(1)$ & $7(1)$ & $13(1)$ & $6(1)$ \\
\hline$C(13)$ & $44(1)$ & $28(1)$ & $30(1)$ & 2(1) & $16(1)$ & $-8(1)$ \\
\hline$C(14)$ & $28(1)$ & $40(1)$ & $27(1)$ & $-6(1)$ & $14(1)$ & $-11(1)$ \\
\hline$C(15)$ & $20(1)$ & $35(1)$ & $23(1)$ & $-3(1)$ & $6(1)$ & $-2(1)$ \\
\hline$C(16)$ & $28(1)$ & $33(1)$ & $20(1)$ & $0(1)$ & $10(1)$ & $-2(1)$ \\
\hline $\mathrm{N}(1)$ & $20(1)$ & $25(1)$ & $20(1)$ & $-4(1)$ & $-4(1)$ & $1(1)$ \\
\hline $\mathrm{O}(1)$ & $15(1)$ & $29(1)$ & $23(1)$ & $-4(1)$ & $-2(1)$ & $3(1)$ \\
\hline $\mathrm{O}(2)$ & $20(1)$ & $30(1)$ & $13(1)$ & $2(1)$ & $3(1)$ & $0(1)$ \\
\hline
\end{tabular}

Table 5. Hydrogen coordinates ( $\left.\times 10^{4}\right)$ and isotropic displacement parameters 
$\left(\AA^{2} \times 10^{3}\right)$ for $\mathbf{S}-27$.

\begin{tabular}{|c|c|c|c|c|}
\hline & $\mathrm{x}$ & $\mathrm{y}$ & $\mathrm{z}$ & $\mathrm{U}(\mathrm{eq})$ \\
\hline $\mathrm{H}(2 \mathrm{~A})$ & $6740(30)$ & $-184(6)$ & $-3840(30)$ & $27(5)$ \\
\hline $\mathrm{H}(2 \mathrm{~B})$ & $8680(30)$ & $-124(6)$ & $-2140(30)$ & $35(5)$ \\
\hline $\mathrm{H}(3 \mathrm{~A})$ & $8550(30)$ & $1223(5)$ & $-250(30)$ & $25(4)$ \\
\hline $\mathrm{H}(3 \mathrm{~B})$ & $6330(30)$ & 1099(5) & $-1460(20)$ & $23(4)$ \\
\hline $\mathrm{H}(4 \mathrm{~A})$ & $7670(20)$ & $1617(5)$ & $-3280(20)$ & $21(4)$ \\
\hline $\mathrm{H}(4 \mathrm{~B})$ & $7050(30)$ & $1209(5)$ & $-4610(30)$ & $27(4)$ \\
\hline $\mathrm{H}(5)$ & $11170(20)$ & $1440(5)$ & $-2520(20)$ & $18(4)$ \\
\hline $\mathrm{H}(6 \mathrm{~A})$ & $12350(30)$ & $829(5)$ & $-3780(30)$ & $25(4)$ \\
\hline $\mathrm{H}(6 \mathrm{~B})$ & $10020(30)$ & $710(5)$ & $-4830(30)$ & $22(4)$ \\
\hline $\mathrm{H}(7 \mathrm{~A})$ & $11240(30)$ & $351(5)$ & $-1620(30)$ & $24(4)$ \\
\hline $\mathrm{H}(7 \mathrm{~B})$ & $11620(30)$ & $784(5)$ & $-500(30)$ & $24(4)$ \\
\hline $\mathrm{H}(8 \mathrm{~A})$ & $9860(30)$ & $1824(5)$ & $-5480(30)$ & $26(4)$ \\
\hline $\mathrm{H}(8 \mathrm{~B})$ & $9320(30)$ & 1401(5) & $-6770(30)$ & $24(4)$ \\
\hline $\mathrm{H}(9 \mathrm{~A})$ & $12870(30)$ & $1257(6)$ & $-6280(30)$ & $34(5)$ \\
\hline $\mathrm{H}(9 \mathrm{~B})$ & $13450(30)$ & 1653(6) & $-4890(30)$ & $42(5)$ \\
\hline $\mathrm{H}(11)$ & $9590(30)$ & $2042(5)$ & $-8620(30)$ & $33(5)$ \\
\hline $\mathrm{H}(12)$ & $9920(30)$ & 2434(7) & $-11470(30)$ & $45(6)$ \\
\hline $\mathrm{H}(13)$ & $12940(30)$ & $2408(6)$ & $-12570(30)$ & $40(5)$ \\
\hline $\mathrm{H}(14)$ & $15760(30)$ & 2002(6) & $-10800(30)$ & $37(5)$ \\
\hline $\mathrm{H}(15)$ & $15470(30)$ & 1611(6) & $-7950(30)$ & $32(5)$ \\
\hline $\mathrm{H}(16 \mathrm{~A})$ & $6490(30)$ & $753(6)$ & $1630(30)$ & $29(5)$ \\
\hline $\mathrm{H}(16 \mathrm{~B})$ & $7110(30)$ & $282(5)$ & $2430(30)$ & $25(4)$ \\
\hline $\mathrm{H}(16 \mathrm{C})$ & $5640(30)$ & $365(6)$ & $160(30)$ & $35(5)$ \\
\hline
\end{tabular}

Table 6. Torsion angles $\left[{ }^{\circ}\right]$ for $\mathbf{S - 2 7}$. 


\begin{tabular}{lc}
\hline $\mathrm{O}(1)-\mathrm{C}(1)-\mathrm{C}(2)-\mathrm{O}(2)$ & $124.37(13)$ \\
$\mathrm{N}(1)-\mathrm{C}(1)-\mathrm{C}(2)-\mathrm{O}(2)$ & $-55.28(15)$ \\
$\mathrm{O}(1)-\mathrm{C}(1)-\mathrm{C}(2)-\mathrm{C}(3)$ & $1.45(18)$ \\
$\mathrm{N}(1)-\mathrm{C}(1)-\mathrm{C}(2)-\mathrm{C}(3)$ & $-178.19(12)$ \\
$\mathrm{O}(1)-\mathrm{C}(1)-\mathrm{C}(2)-\mathrm{C}(7)$ & $-121.49(14)$ \\
$\mathrm{N}(1)-\mathrm{C}(1)-\mathrm{C}(2)-\mathrm{C}(7)$ & $58.87(16)$ \\
$\mathrm{O}(2)-\mathrm{C}(2)-\mathrm{C}(3)-\mathrm{C}(4)$ & $166.80(11)$ \\
$\mathrm{C}(7)-\mathrm{C}(2)-\mathrm{C}(3)-\mathrm{C}(4)$ & $50.96(15)$ \\
$\mathrm{C}(1)-\mathrm{C}(2)-\mathrm{C}(3)-\mathrm{C}(4)$ & $-72.63(15)$ \\
$\mathrm{C}(2)-\mathrm{C}(3)-\mathrm{C}(4)-\mathrm{C}(5)$ & $-51.56(17)$ \\
$\mathrm{C}(3)-\mathrm{C}(4)-\mathrm{C}(5)-\mathrm{C}(6)$ & $53.06(16)$ \\
$\mathrm{C}(3)-\mathrm{C}(4)-\mathrm{C}(5)-\mathrm{C}(8)$ & $178.29(12)$ \\
$\mathrm{C}(4)-\mathrm{C}(5)-\mathrm{C}(6)-\mathrm{C}(7)$ & $-56.31(15)$ \\
$\mathrm{C}(8)-\mathrm{C}(5)-\mathrm{C}(6)-\mathrm{C}(7)$ & $-178.80(12)$ \\
$\mathrm{C}(5)-\mathrm{C}(6)-\mathrm{C}(7)-\mathrm{C}(2)$ & $59.28(16)$ \\
$\mathrm{O}(2)-\mathrm{C}(2)-\mathrm{C}(7)-\mathrm{C}(6)$ & $-175.46(11)$ \\
$\mathrm{C}(3)-\mathrm{C}(2)-\mathrm{C}(7)-\mathrm{C}(6)$ & $-55.33(15)$ \\
$\mathrm{C}(1)-\mathrm{C}(2)-\mathrm{C}(7)-\mathrm{C}(6)$ & $68.62(16)$ \\
$\mathrm{C}(6)-\mathrm{C}(5)-\mathrm{C}(8)-\mathrm{C}(9)$ & $-58.35(18)$ \\
$\mathrm{C}(4)-\mathrm{C}(5)-\mathrm{C}(8)-\mathrm{C}(9)$ & $178.94(13)$ \\
$\mathrm{C}(5)-\mathrm{C}(8)-\mathrm{C}(9)-\mathrm{C}(10)$ & $-176.75(13)$ \\
$\mathrm{C}(8)-\mathrm{C}(9)-\mathrm{C}(10)-\mathrm{C}(11)$ & $7.7(2)$ \\
$\mathrm{C}(8)-\mathrm{C}(9)-\mathrm{C}(10)-\mathrm{C}(15)$ & $-172.82(14)$ \\
$\mathrm{C}(15)-\mathrm{C}(10)-\mathrm{C}(11)-\mathrm{C}(12)$ & $-0.4(2)$ \\
$\mathrm{C}(9)-\mathrm{C}(10)-\mathrm{C}(11)-\mathrm{C}(12)$ & $179.10(16)$ \\
$\mathrm{C}(10)-\mathrm{C}(11)-\mathrm{C}(12)-\mathrm{C}(13)$ & $-0.1(3)$ \\
$\mathrm{C}(11)-\mathrm{C}(12)-\mathrm{C}(13)-\mathrm{C}(14)$ & $0.5(3)$ \\
$\mathrm{C}(12)-\mathrm{C}(13)-\mathrm{C}(14)-\mathrm{C}(15)$ & $-0.4(3)$ \\
$\mathrm{C}(13)-\mathrm{C}(14)-\mathrm{C}(15)-\mathrm{C}(10)$ & $-0.1(2)$ \\
$\mathrm{C}(11)-\mathrm{C}(10)-\mathrm{C}(15)-\mathrm{C}(14)$ & $0.5(2)$ \\
$\mathrm{C}(9)-\mathrm{C}(10)-\mathrm{C}(15)-\mathrm{C}(14)$ & $-179.04(15)$ \\
$\mathrm{C}(3)-\mathrm{C}(2)-\mathrm{O}(2)-\mathrm{C}(16)$ & $57.52(15)$ \\
$\mathrm{C}(7)-\mathrm{C}(2)-\mathrm{O}(2)-\mathrm{C}(16)$ & $176.41(12)$ \\
$\mathrm{C}(1)-\mathrm{C}(2)-\mathrm{O}(2)-\mathrm{C}(16)$ & $-65.35(14)$ \\
\hline
\end{tabular}




\section{Alcohol S-28}

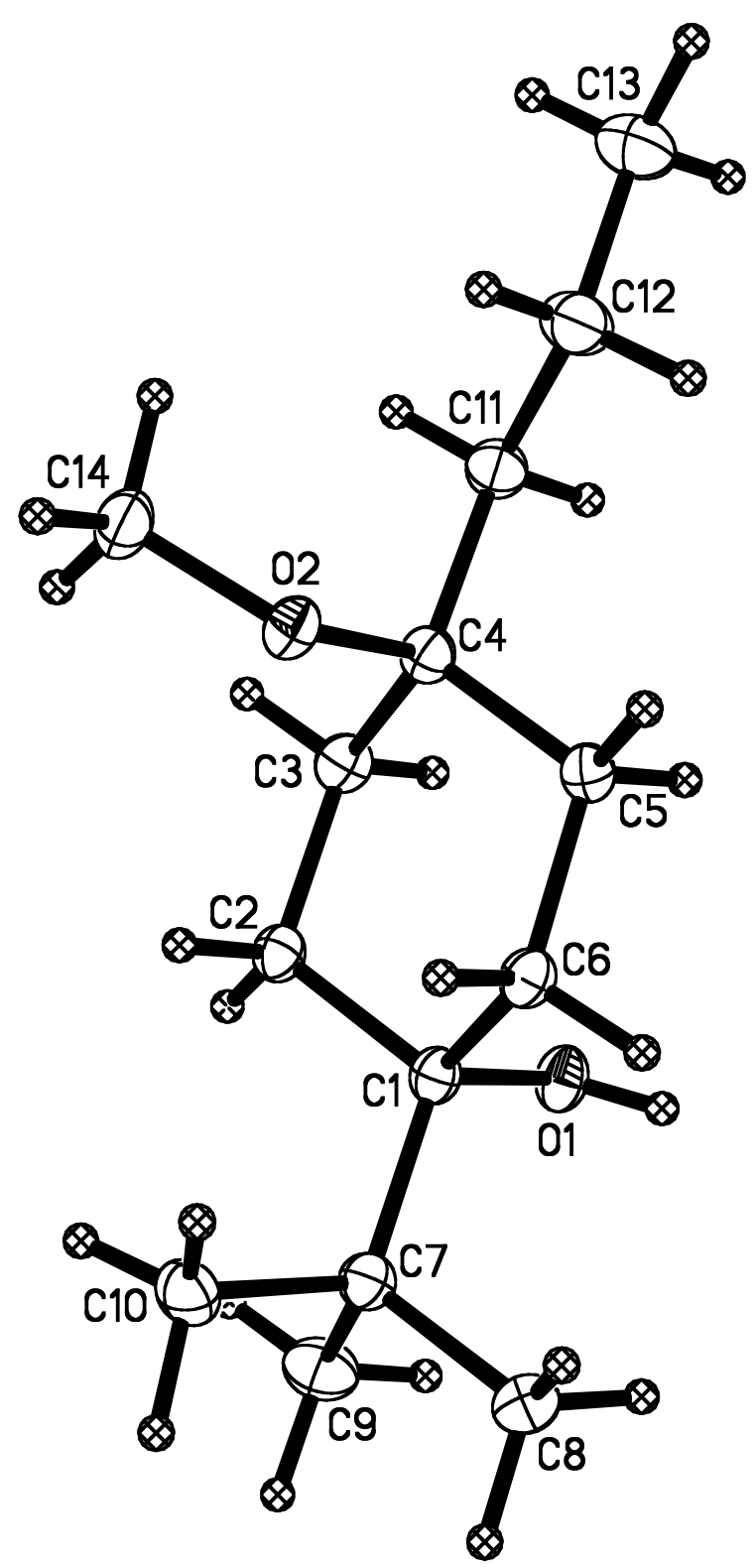

X-ray Data Collection, Structure Solution and Refinement for S-28.

A colorless crystal of approximate dimensions $0.27 \times 0.33 \times 0.41 \mathrm{~mm}$ was mounted on a glass fiber and transferred to a Bruker CCD platform diffractometer. The SMART ${ }^{1}$ program package was used to determine the unit-cell parameters and for data collection $(20 \mathrm{sec} /$ frame scan time for a sphere of diffraction data). The raw frame data was 
processed using $\mathrm{SAINT}^{2}$ and $\mathrm{SADABS}^{3}$ to yield the reflection data file. Subsequent calculations were carried out using the SHELXTL ${ }^{4}$ program. The diffraction symmetry was $2 / m$ and the systematic absences were consistent with the centrosymmetric monoclinic space group $P 2_{1} / c$ which was later determined to be correct.

The structure was solved by direct methods and refined on $\mathrm{F}^{2}$ by full-matrix least-squares techniques. The analytical scattering factors ${ }^{5}$ for neutral atoms were used throughout the analysis. Hydrogen atoms were located from a difference-Fourier map and refined (x,y,z and $U_{\text {iso }}$ ). At convergence, $w R 2=0.1038$ and $\mathrm{Goof}=1.031$ for 257 variables refined against 3432 data. As a comparison for refinement on F, R1 $=0.0352$ for those 2940 data with I $>2.0 \sigma(\mathrm{I})$.

Definitions:

$$
\mathrm{wR} 2=\left[\Sigma\left[\mathrm{w}\left(\mathrm{F}_{\mathrm{o}}^{2}-\mathrm{F}_{\mathrm{c}}\right)^{2}\right] / \Sigma\left[\mathrm{w}\left(\mathrm{F}_{\mathrm{o}}^{2}\right)^{2}\right]\right]^{1 / 2}
$$

$\mathrm{R} 1=\Sigma|| \mathrm{F}_{\mathrm{o}}|-| \mathrm{F}_{\mathrm{c}}|| / \Sigma\left|\mathrm{F}_{\mathrm{o}}\right|$

Goof $=\mathrm{S}=\left[\Sigma\left[\mathrm{w}\left(\mathrm{F}_{\mathrm{o}}^{2}-\mathrm{F}_{\mathrm{c}}{ }^{2}\right)^{2}\right] /(\mathrm{n}-\mathrm{p})\right]^{1 / 2}$ where $\mathrm{n}$ is the number of reflections and $\mathrm{p}$ is the total

number of parameters refined.

The thermal ellipsoid plot is shown at the $50 \%$ probability level.

Table 1. Crystal data and structure refinement for $\mathbf{S - 2 8}$.

Identification code

Empirical formula

Temperature

Formula weight

Wavelength

Crystal system

Space group

Unit cell dimensions

Volume

Z

Density (calculated)

Absorption coefficient

$\mathrm{F}(000)$

Crystal color

Crystal size
S-28 (Glen Baghdasarian)

228.36

$\mathrm{C}_{14} \mathrm{H}_{28} \mathrm{O}_{2}$

163(2) K

$0.71073 \AA$

Monoclinic

$P 2{ }_{1} / c$

$$
\begin{array}{ll}
\mathrm{a}=7.5049(6) \AA & \alpha=90^{\circ} . \\
\mathrm{b}=9.4498(7) \AA & \beta=94.6760(10)^{\circ} . \\
\mathrm{c}=19.6691(16) \AA & \gamma=90^{\circ} .
\end{array}
$$

$1390.29(19) \AA^{3}$

4

$1.091 \mathrm{Mg} / \mathrm{m}^{3}$

$0.070 \mathrm{~mm}^{-1}$

512

colorless

$0.41 \times 0.33 \times 0.27 \mathrm{~mm}^{3}$ 
Theta range for data collection

Index ranges

Reflections collected

Independent reflections

Completeness to theta $=28.37^{\circ}$

Absorption correction

Max. and min. transmission

Refinement method

Data / restraints / parameters

Goodness-of-fit on $\mathrm{F}^{2}$

Final $R$ indices $[\mathrm{I}>2 \operatorname{sigma}(\mathrm{I})=2940$ data]

$\mathrm{R}$ indices (all data)

Largest diff. peak and hole
2.08 to $28.37^{\circ}$.

$-10 \leq h \leq 9,-12 \leq k \leq 12,-26 \leq l \leq 26$

17373

$3432[\mathrm{R}(\mathrm{int})=0.0238]$

$98.6 \%$

Semi-empirical from equivalents

0.9813 and 0.9718

Full-matrix least-squares on $\mathrm{F}^{2}$

3432 / 0 / 257

1.031

$\mathrm{R} 1=0.0352, \mathrm{wR} 2=0.0965$

$\mathrm{R} 1=0.0426, \mathrm{wR} 2=0.1038$

0.435 and -0.186 e. $\AA^{-3}$

Table 2. Atomic coordinates $\left(\mathrm{x} 10^{4}\right)$ and equivalent isotropic displacement parameters $\left(\AA^{2} \times 10^{3}\right)$

for $\mathbf{S - 2 8}$. U(eq) is defined as one third of the trace of the orthogonalized $U^{i j}$ tensor.

\begin{tabular}{|c|c|c|c|c|}
\hline & $\mathrm{x}$ & $\mathrm{y}$ & $\mathrm{z}$ & $\mathrm{U}(\mathrm{eq})$ \\
\hline $\mathrm{O}(1)$ & $2876(1)$ & $215(1)$ & $2750(1)$ & $20(1)$ \\
\hline $\mathrm{O}(2)$ & $4351(1)$ & $3783(1)$ & $1390(1)$ & $18(1)$ \\
\hline $\mathrm{C}(1)$ & $2682(1)$ & $1730(1)$ & $2732(1)$ & $16(1)$ \\
\hline$C(2)$ & $1639(1)$ & $2046(1)$ & $2042(1)$ & $17(1)$ \\
\hline$C(3)$ & $2646(1)$ & $1556(1)$ & $1441(1)$ & $18(1)$ \\
\hline$C(4)$ & $4492(1)$ & $2247(1)$ & $1430(1)$ & $16(1)$ \\
\hline$C(5)$ & $5548(1)$ & $1980(1)$ & $2118(1)$ & $17(1)$ \\
\hline$C(6)$ & $4539(1)$ & $2418(1)$ & $2733(1)$ & $17(1)$ \\
\hline$C(7)$ & $1620(1)$ & $2214(1)$ & $3347(1)$ & $18(1)$ \\
\hline$C(8)$ & $2788(1)$ & $2064(1)$ & $4021(1)$ & $25(1)$ \\
\hline$C(9)$ & $-33(1)$ & $1276(1)$ & $3397(1)$ & $26(1)$ \\
\hline$C(10)$ & 991(1) & $3756(1)$ & $3276(1)$ & $23(1)$ \\
\hline $\mathrm{C}(11)$ & $5455(1)$ & $1655(1)$ & $832(1)$ & $21(1)$ \\
\hline$C(12)$ & $7251(1)$ & $2324(1)$ & $710(1)$ & $27(1)$ \\
\hline
\end{tabular}


$\mathrm{C}(13)$

$\begin{array}{llrl}8061(2) & 1667(2) & 98(1) & 37(1) \\ 3384(1) & 4337(1) & 798(1) & 23(1)\end{array}$

Table 3. Bond lengths $[\AA]$ and angles $\left[{ }^{\circ}\right]$ for $\mathbf{S - 2 8}$.

\begin{tabular}{lr}
\hline $\mathrm{O}(1)-\mathrm{C}(1)$ & $1.4392(10)$ \\
$\mathrm{O}(2)-\mathrm{C}(14)$ & $1.4216(10)$ \\
$\mathrm{O}(2)-\mathrm{C}(4)$ & $1.4574(10)$ \\
$\mathrm{C}(1)-\mathrm{C}(6)$ & $1.5382(12)$ \\
$\mathrm{C}(1)-\mathrm{C}(2)$ & $1.5385(11)$ \\
$\mathrm{C}(1)-\mathrm{C}(7)$ & $1.5702(12)$ \\
$\mathrm{C}(2)-\mathrm{C}(3)$ & $1.5268(12)$ \\
$\mathrm{C}(3)-\mathrm{C}(4)$ & $1.5333(12)$ \\
$\mathrm{C}(4)-\mathrm{C}(5)$ & $1.5328(11)$ \\
$\mathrm{C}(4)-\mathrm{C}(11)$ & $1.5356(12)$ \\
$\mathrm{C}(5)-\mathrm{C}(6)$ & $1.5345(12)$ \\
$\mathrm{C}(7)-\mathrm{C}(10)$ & $1.5341(12)$ \\
$\mathrm{C}(7)-\mathrm{C}(9)$ & $1.5348(13)$ \\
$\mathrm{C}(7)-\mathrm{C}(8)$ & $1.5360(12)$ \\
$\mathrm{C}(11)-\mathrm{C}(12)$ & $1.5251(13)$ \\
$\mathrm{C}(12)-\mathrm{C}(13)$ & $1.5244(14)$ \\
$\mathrm{C}(14)-\mathrm{O}(2)-\mathrm{C}(4)$ & $116.18(7)$ \\
$\mathrm{O}(1)-\mathrm{C}(1)-\mathrm{C}(6)$ & $109.33(7)$ \\
$\mathrm{O}(1)-\mathrm{C}(1)-\mathrm{C}(2)$ & $105.02(7)$ \\
$\mathrm{C}(6)-\mathrm{C}(1)-\mathrm{C}(2)$ & $108.37(7)$ \\
$\mathrm{O}(1)-\mathrm{C}(1)-\mathrm{C}(7)$ & $109.10(7)$ \\
$\mathrm{C}(6)-\mathrm{C}(1)-\mathrm{C}(7)$ & $113.07(7)$ \\
$\mathrm{C}(2)-\mathrm{C}(1)-\mathrm{C}(7)$ & $111.62(7)$ \\
$\mathrm{C}(3)-\mathrm{C}(2)-\mathrm{C}(1)$ & $112.03(7)$ \\
$\mathrm{C}(2)-\mathrm{C}(3)-\mathrm{C}(4)$ & $112.76(7)$ \\
$\mathrm{O}(2)-\mathrm{C}(4)-\mathrm{C}(5)$ & $103.96(6)$ \\
$\mathrm{O}(2)-\mathrm{C}(4)-\mathrm{C}(3)$ & $111.28(7)$ \\
$\mathrm{C}(5)-\mathrm{C}(4)-\mathrm{C}(3)$ & $108.66(7)$ \\
$\mathrm{O}(2)-\mathrm{C}(4)-\mathrm{C}(11)$ & $110.98(7)$ \\
&
\end{tabular}




$\begin{array}{ll}\mathrm{C}(5)-\mathrm{C}(4)-\mathrm{C}(11) & 112.08(7) \\ \mathrm{C}(3)-\mathrm{C}(4)-\mathrm{C}(11) & 109.76(7) \\ \mathrm{C}(4)-\mathrm{C}(5)-\mathrm{C}(6) & 113.52(7) \\ \mathrm{C}(5)-\mathrm{C}(6)-\mathrm{C}(1) & 112.92(7) \\ \mathrm{C}(10)-\mathrm{C}(7)-\mathrm{C}(9) & 108.01(8) \\ \mathrm{C}(10)-\mathrm{C}(7)-\mathrm{C}(8) & 108.40(8) \\ \mathrm{C}(9)-\mathrm{C}(7)-\mathrm{C}(8) & 107.34(8) \\ \mathrm{C}(10)-\mathrm{C}(7)-\mathrm{C}(1) & 112.29(7) \\ \mathrm{C}(9)-\mathrm{C}(7)-\mathrm{C}(1) & 110.19(7) \\ \mathrm{C}(8)-\mathrm{C}(7)-\mathrm{C}(1) & 110.45(7) \\ \mathrm{C}(12)-\mathrm{C}(11)-\mathrm{C}(4) & 116.48(8) \\ \mathrm{C}(13)-\mathrm{C}(12)-\mathrm{C}(11) & 111.64(9)\end{array}$

Table 4. Anisotropic displacement parameters $\left(\AA^{2} \times 10^{3}\right)$ for S-28. The anisotropic displacement factor exponent takes the form: $-2 \pi^{2}\left[h^{2} a^{* 2} U^{11}+\ldots+2 h k a^{*} b^{*} U^{12}\right]$

\begin{tabular}{|c|c|c|c|c|c|c|}
\hline & $U^{11}$ & $\mathrm{U}^{22}$ & $U^{33}$ & $\mathrm{U}^{23}$ & $U^{13}$ & $\mathrm{U}^{12}$ \\
\hline $\mathrm{O}(1)$ & $22(1)$ & $13(1)$ & $23(1)$ & 1(1) & $-2(1)$ & 1(1) \\
\hline $\mathrm{O}(2)$ & 21(1) & $15(1)$ & $17(1)$ & 1(1) & $-1(1)$ & 1(1) \\
\hline$C(1)$ & $17(1)$ & $13(1)$ & $17(1)$ & 1(1) & $0(1)$ & $1(1)$ \\
\hline$C(2)$ & $16(1)$ & $17(1)$ & $17(1)$ & 1(1) & $0(1)$ & $0(1)$ \\
\hline$C(3)$ & 19(1) & $18(1)$ & $17(1)$ & $-2(1)$ & $-1(1)$ & $-2(1)$ \\
\hline $\mathrm{C}(4)$ & $18(1)$ & $15(1)$ & $16(1)$ & $-1(1)$ & $1(1)$ & $1(1)$ \\
\hline$C(5)$ & $16(1)$ & $17(1)$ & $18(1)$ & $0(1)$ & $0(1)$ & $1(1)$ \\
\hline$C(6)$ & $17(1)$ & $16(1)$ & $16(1)$ & $0(1)$ & $-1(1)$ & $-1(1)$ \\
\hline$C(7)$ & $19(1)$ & $17(1)$ & $18(1)$ & $0(1)$ & $2(1)$ & $1(1)$ \\
\hline $\mathrm{C}(8)$ & $27(1)$ & $33(1)$ & $17(1)$ & $0(1)$ & 2(1) & $5(1)$ \\
\hline C(9) & $25(1)$ & $27(1)$ & $29(1)$ & $-1(1)$ & $9(1)$ & $-5(1)$ \\
\hline$C(10)$ & $25(1)$ & 19(1) & $26(1)$ & $-1(1)$ & $6(1)$ & $4(1)$ \\
\hline $\mathrm{C}(11)$ & $23(1)$ & $21(1)$ & $18(1)$ & $-2(1)$ & 2(1) & $2(1)$ \\
\hline $\mathrm{C}(12)$ & $23(1)$ & $35(1)$ & $24(1)$ & $-1(1)$ & $6(1)$ & $1(1)$ \\
\hline$C(13)$ & $32(1)$ & $52(1)$ & $27(1)$ & $1(1)$ & $12(1)$ & $8(1)$ \\
\hline
\end{tabular}


$\mathrm{C}(14)$

$22(1)$

20(1)

$3(1)$

$-2(1)$

$4(1)$

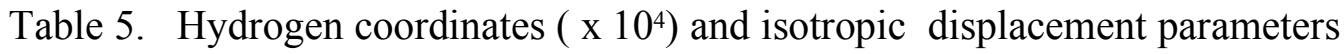
$\left(\AA^{2} \times 10^{3}\right)$ for $\mathbf{S}-\mathbf{2 8}$.

\begin{tabular}{|c|c|c|c|c|}
\hline & $\mathrm{x}$ & $\mathrm{y}$ & $\mathrm{z}$ & $\mathrm{U}(\mathrm{eq})$ \\
\hline $\mathrm{H}(1)$ & $3690(20)$ & $-9(15)$ & $3024(7)$ & $37(4)$ \\
\hline $\mathrm{H}(2 \mathrm{~A})$ & $456(16)$ & $1587(13)$ & $2011(6)$ & $23(3)$ \\
\hline $\mathrm{H}(2 \mathrm{~B})$ & $1433(15)$ & $3059(12)$ & $2001(6)$ & $18(3)$ \\
\hline $\mathrm{H}(3 \mathrm{~A})$ & $2816(15)$ & $542(13)$ & $1465(6)$ & $23(3)$ \\
\hline $\mathrm{H}(3 \mathrm{~B})$ & $1946(16)$ & 1731(13) & $1006(6)$ & $23(3)$ \\
\hline $\mathrm{H}(5 \mathrm{~A})$ & $5804(15)$ & $960(13)$ & $2149(6)$ & $21(3)$ \\
\hline $\mathrm{H}(5 \mathrm{~B})$ & $6694(15)$ & $2497(13)$ & $2141(6)$ & $19(3)$ \\
\hline $\mathrm{H}(6 \mathrm{~A})$ & $4436(15)$ & $3432(13)$ & $2742(6)$ & $21(3)$ \\
\hline $\mathrm{H}(6 \mathrm{~B})$ & $5253(15)$ & $2160(12)$ & $3152(6)$ & $20(3)$ \\
\hline $\mathrm{H}(8 \mathrm{~A})$ & 2057(19) & $2272(15)$ & $4406(7)$ & $39(4)$ \\
\hline $\mathrm{H}(8 \mathrm{~B})$ & $3272(18)$ & $1105(15)$ & $4087(7)$ & $36(3)$ \\
\hline $\mathrm{H}(8 \mathrm{C})$ & $3825(19)$ & $2735(15)$ & $4056(7)$ & $34(3)$ \\
\hline $\mathrm{H}(9 \mathrm{~A})$ & $-692(18)$ & 1594(14) & $3777(7)$ & $32(3)$ \\
\hline H(9B) & $300(20)$ & $296(17)$ & $3491(7)$ & $45(4)$ \\
\hline $\mathrm{H}(9 \mathrm{C})$ & $-838(18)$ & $1307(15)$ & $2983(7)$ & $37(3)$ \\
\hline $\mathrm{H}(10 \mathrm{~A})$ & $467(18)$ & $4052(15)$ & $3704(7)$ & $36(3)$ \\
\hline $\mathrm{H}(10 \mathrm{~B})$ & $55(18)$ & $3850(14)$ & $2910(7)$ & $31(3)$ \\
\hline $\mathrm{H}(10 \mathrm{C})$ & $1956(18)$ & $4435(15)$ & $3194(7)$ & $34(3)$ \\
\hline $\mathrm{H}(11 \mathrm{~A})$ & $5598(16)$ & $632(13)$ & $913(6)$ & $23(3)$ \\
\hline $\mathrm{H}(11 \mathrm{~B})$ & $4653(17)$ & 1741(13) & $409(6)$ & $27(3)$ \\
\hline $\mathrm{H}(12 \mathrm{~A})$ & $8090(20)$ & $2226(16)$ & $1125(7)$ & $44(4)$ \\
\hline $\mathrm{H}(12 \mathrm{~B})$ & $7109(17)$ & $3311(15)$ & $636(7)$ & $31(3)$ \\
\hline $\mathrm{H}(13 \mathrm{~A})$ & $7260(20)$ & $1760(16)$ & $-317(8)$ & $49(4)$ \\
\hline $\mathrm{H}(13 \mathrm{~B})$ & $9230(20)$ & $2077(17)$ & $2(8)$ & $48(4)$ \\
\hline
\end{tabular}




$\begin{array}{lrrrr}\mathrm{H}(13 \mathrm{C}) & 8270(20) & 661(19) & 164(8) & 54(4) \\ \mathrm{H}(14 \mathrm{~A}) & 3943(18) & 4091(14) & 387(7) & 34(3) \\ \mathrm{H}(14 \mathrm{~B}) & 2149(18) & 4018(14) & 753(6) & 33(3) \\ \mathrm{H}(14 \mathrm{C}) & 3383(16) & 5359(14) & 846(6) & 25(3)\end{array}$

Table 6. Torsion angles $\left[^{\circ}\right]$ for $\mathbf{S - 2 8}$.

\begin{tabular}{lc}
\hline $\mathrm{O}(1)-\mathrm{C}(1)-\mathrm{C}(2)-\mathrm{C}(3)$ & $-60.94(9)$ \\
$\mathrm{C}(6)-\mathrm{C}(1)-\mathrm{C}(2)-\mathrm{C}(3)$ & $55.81(9)$ \\
$\mathrm{C}(7)-\mathrm{C}(1)-\mathrm{C}(2)-\mathrm{C}(3)$ & $-179.03(7)$ \\
$\mathrm{C}(1)-\mathrm{C}(2)-\mathrm{C}(3)-\mathrm{C}(4)$ & $-58.60(9)$ \\
$\mathrm{C}(14)-\mathrm{O}(2)-\mathrm{C}(4)-\mathrm{C}(5)$ & $-178.73(7)$ \\
$\mathrm{C}(14)-\mathrm{O}(2)-\mathrm{C}(4)-\mathrm{C}(3)$ & $-61.94(9)$ \\
$\mathrm{C}(14)-\mathrm{O}(2)-\mathrm{C}(4)-\mathrm{C}(11)$ & $60.60(9)$ \\
$\mathrm{C}(2)-\mathrm{C}(3)-\mathrm{C}(4)-\mathrm{O}(2)$ & $-59.24(9)$ \\
$\mathrm{C}(2)-\mathrm{C}(3)-\mathrm{C}(4)-\mathrm{C}(5)$ & $54.64(9)$ \\
$\mathrm{C}(2)-\mathrm{C}(3)-\mathrm{C}(4)-\mathrm{C}(11)$ & $177.53(7)$ \\
$\mathrm{O}(2)-\mathrm{C}(4)-\mathrm{C}(5)-\mathrm{C}(6)$ & $66.04(9)$ \\
$\mathrm{C}(3)-\mathrm{C}(4)-\mathrm{C}(5)-\mathrm{C}(6)$ & $-52.55(9)$ \\
$\mathrm{C}(11)-\mathrm{C}(4)-\mathrm{C}(5)-\mathrm{C}(6)$ & $-174.02(7)$ \\
$\mathrm{C}(4)-\mathrm{C}(5)-\mathrm{C}(6)-\mathrm{C}(1)$ & $54.51(10)$ \\
$\mathrm{O}(1)-\mathrm{C}(1)-\mathrm{C}(6)-\mathrm{C}(5)$ & $60.13(9)$ \\
$\mathrm{C}(2)-\mathrm{C}(1)-\mathrm{C}(6)-\mathrm{C}(5)$ & $-53.81(9)$ \\
$\mathrm{C}(7)-\mathrm{C}(1)-\mathrm{C}(6)-\mathrm{C}(5)$ & $-178.11(7)$ \\
$\mathrm{O}(1)-\mathrm{C}(1)-\mathrm{C}(7)-\mathrm{C}(10)$ & $-167.15(7)$ \\
$\mathrm{C}(6)-\mathrm{C}(1)-\mathrm{C}(7)-\mathrm{C}(10)$ & $70.96(9)$ \\
$\mathrm{C}(2)-\mathrm{C}(1)-\mathrm{C}(7)-\mathrm{C}(10)$ & $-51.54(10)$ \\
$\mathrm{O}(1)-\mathrm{C}(1)-\mathrm{C}(7)-\mathrm{C}(9)$ & $-46.70(9)$ \\
$\mathrm{C}(6)-\mathrm{C}(1)-\mathrm{C}(7)-\mathrm{C}(9)$ & $-168.58(7)$ \\
$\mathrm{C}(2)-\mathrm{C}(1)-\mathrm{C}(7)-\mathrm{C}(9)$ & $68.92(9)$ \\
$\mathrm{O}(1)-\mathrm{C}(1)-\mathrm{C}(7)-\mathrm{C}(8)$ & $71.72(9)$ \\
$\mathrm{C}(6)-\mathrm{C}(1)-\mathrm{C}(7)-\mathrm{C}(8)$ & $-50.17(10)$ \\
$\mathrm{C}(2)-\mathrm{C}(1)-\mathrm{C}(7)-\mathrm{C}(8)$ & $-172.67(8)$ \\
$\mathrm{O}(2)-\mathrm{C}(4)-\mathrm{C}(11)-\mathrm{C}(12)$ & \\
& \\
&
\end{tabular}



$\mathrm{C}(5)-\mathrm{C}(4)-\mathrm{C}(11)-\mathrm{C}(12)$
$\mathrm{C}(3)-\mathrm{C}(4)-\mathrm{C}(11)-\mathrm{C}(12)$
$\mathrm{C}(4)-\mathrm{C}(11)-\mathrm{C}(12)-\mathrm{C}(13)$
$-179.14(9)$

\section{Alcohol S-29}
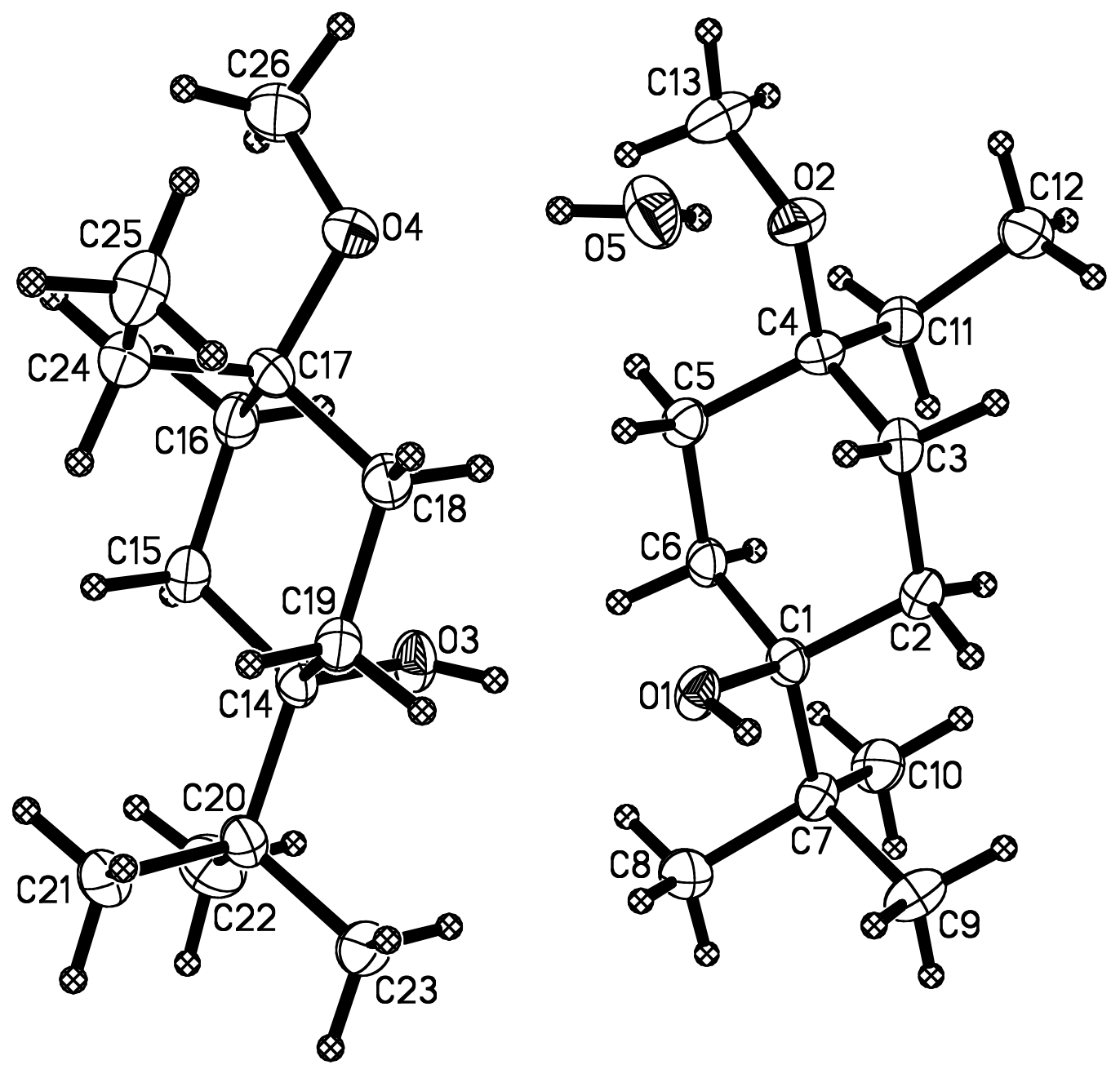

X-ray Data Collection, Structure Solution and Refinement for S-29.

A colorless crystal of approximate dimensions $0.09 \times 0.23 \times 0.28 \mathrm{~mm}$ was mounted on a glass fiber and transferred to a Bruker CCD platform diffractometer. The SMART ${ }^{1}$ program package was used to determine the unit-cell parameters and for data collection $(25 \mathrm{sec} /$ frame scan time for a sphere of diffraction data). The raw frame data was processed using SAINT ${ }^{2}$ and SADABS $^{3}$ to yield the reflection data file. Subsequent 
calculations were carried out using the SHELXTL ${ }^{4}$ program. The diffraction symmetry was $2 / m$ and the systematic absences were consistent with the centrosymmetric monoclinic space group $P 2_{1} / c$ which was later determined to be correct.

The structure was solved by direct methods and refined on $\mathrm{F}^{2}$ by full-matrix least-squares techniques. The analytical scattering factors ${ }^{5}$ for neutral atoms were used throughout the analysis. Hydrogen atoms were included using a riding model. There were two molecules of the formula unit present. There was also one-half molecule of water present per formula unit. At convergence, $w R 2=0.1295$ and Goof $=1.058$ for 297 variables refined against 3941 data $(0.90 \AA)$. As a comparison for refinement on $\mathrm{F}, \mathrm{R} 1=0.0482$ for those 2283 data with $\mathrm{I}>2.0 \sigma(\mathrm{I})$.

Definitions:

$$
\mathrm{wR} 2=\left[\Sigma\left[\mathrm{w}\left(\mathrm{F}_{\mathrm{o}}^{2}-\mathrm{F}_{\mathrm{c}}\right)^{2}\right] / \Sigma\left[\mathrm{w}\left(\mathrm{F}_{\mathrm{o}}^{2}\right)^{2}\right]\right]^{1 / 2}
$$

$\mathrm{R} 1=\Sigma|| \mathrm{F}_{\mathrm{o}}|-| \mathrm{F}_{\mathrm{c}}|| / \Sigma\left|\mathrm{F}_{\mathrm{o}}\right|$

Goof $=\mathrm{S}=\left[\Sigma\left[\mathrm{w}\left(\mathrm{F}_{\mathrm{o}}^{2}-\mathrm{F}_{\mathrm{c}}^{2}\right)^{2}\right] /(\mathrm{n}-\mathrm{p})\right]^{1 / 2}$ where $\mathrm{n}$ is the number of reflections and $\mathrm{p}$ is the total

number of parameters refined.

The thermal ellipsoid plot is shown at the $40 \%$ probability level.

Table 1. Crystal data and structure refinement for S-29.

Identification code

Empirical formula

Formula weight

Temperature

Wavelength

Crystal system

Space group

Unit cell dimensions

Volume

Z

Density (calculated)

Absorption coefficient

$\mathrm{F}(000)$

Crystal color

Crystal size
S-29 (Glen Baghdasarian)

$\mathrm{C}_{13} \mathrm{H}_{26} \mathrm{O}_{2} \cdot 1 / 2\left(\mathrm{H}_{2} \mathrm{O}\right)$

223.35

173(2) K

$0.71073 \AA$

Monoclinic

$P 2{ }_{1} / c$

$$
\begin{array}{ll}
\mathrm{a}=11.812(3) \AA & \alpha=90^{\circ} . \\
\mathrm{b}=10.601(3) \AA & \beta=100.453(5)^{\circ} . \\
\mathrm{c}=22.291(6) \AA & \gamma=90^{\circ} .
\end{array}
$$

2745.1(12) $\AA^{3}$

8

$1.081 \mathrm{Mg} / \mathrm{m}^{3}$

$0.072 \mathrm{~mm}^{-1}$

1000

colorless

$0.28 \times 0.23 \times 0.09 \mathrm{~mm}^{3}$ 
Theta range for data collection

Index ranges

Reflections collected

Independent reflections

Completeness to theta $=23.25^{\circ}$

Absorption correction

Max. and min. transmission

Refinement method

Data / restraints / parameters

Goodness-of-fit on $\mathrm{F}^{2}$

Final $\mathrm{R}$ indices $[\mathrm{I}>2 \operatorname{sigma}(\mathrm{I})=2283$ data $]$

$\mathrm{R}$ indices (all data; $0.90 \AA$ )

Extinction coefficient

Largest diff. peak and hole
1.75 to $23.25^{\circ}$.

$-13 \leq h \leq 13,-11 \leq k \leq 11,-24 \leq l \leq 24$

19886

$3941[\mathrm{R}(\mathrm{int})=0.0848]$

$100.0 \%$

Semi-empirical from equivalents

0.9935 and 0.9801

Full-matrix least-squares on $\mathrm{F}^{2}$

3941 / 0 / 297

1.058

$\mathrm{R} 1=0.0482, \mathrm{wR} 2=0.0985$

$\mathrm{R} 1=0.1141, \mathrm{wR} 2=0.1295$

$0.0029(4)$

0.214 and -0.198 e. $\AA^{-3}$

Table 2. Atomic coordinates ( $\mathrm{x} 10^{4}$ ) and equivalent isotropic displacement parameters $\left(\AA^{2} \times 10^{3}\right)$

for S-29. U(eq) is defined as one third of the trace of the orthogonalized Uij tensor.

\begin{tabular}{lcccc}
\hline & & & \\
& & & \\
& & & \\
& & & & \\
& & & & \\
\hline $\mathrm{O}(1)$ & & & & \\
$\mathrm{O}(2)$ & $5324(2)$ & $2915(2)$ & $1426(1)$ & $28(1)$ \\
$\mathrm{C}(1)$ & $5713(2)$ & $7124(2)$ & $1826(1)$ & $35(1)$ \\
$\mathrm{C}(2)$ & $6241(2)$ & $3412(3)$ & $1142(1)$ & $26(1)$ \\
$\mathrm{C}(3)$ & $7141(2)$ & $4025(3)$ & $1636(1)$ & $27(1)$ \\
$\mathrm{C}(4)$ & $6694(3)$ & $5188(3)$ & $1930(1)$ & $29(1)$ \\
$\mathrm{C}(5)$ & $6183(3)$ & $6180(3)$ & $1462(1)$ & $28(1)$ \\
$\mathrm{C}(6)$ & $5225(3)$ & $5551(3)$ & $1011(1)$ & $28(1)$ \\
$\mathrm{C}(7)$ & $5659(3)$ & $4414(3)$ & $696(1)$ & $28(1)$ \\
$\mathrm{C}(8)$ & $6765(2)$ & $2313(3)$ & $807(1)$ & $28(1)$ \\
$\mathrm{C}(9)$ & $5801(3)$ & $1543(3)$ & $411(2)$ & $41(1)$ \\
$\mathrm{C}(10)$ & $7462(3)$ & $1398(3)$ & $1265(2)$ & $41(1)$ \\
$\mathrm{C}(11)$ & $7559(3)$ & $2813(3)$ & $382(2)$ & $39(1)$ \\
& $7079(3)$ & $6800(3)$ & $1137(2)$ & $32(1)$
\end{tabular}


Supporting Information: Glen Baghdasarian and K. A. Woerpel

\begin{tabular}{lrrrr}
$\mathrm{C}(12)$ & $7965(3)$ & $7622(3)$ & $1546(2)$ & $40(1)$ \\
$\mathrm{C}(13)$ & $5115(3)$ & $8169(3)$ & $1503(2)$ & $47(1)$ \\
$\mathrm{O}(3)$ & $3129(2)$ & $3087(2)$ & $737(1)$ & $35(1)$ \\
$\mathrm{O}(4)$ & $2014(2)$ & $6328(2)$ & $1751(1)$ & $40(1)$ \\
$\mathrm{C}(14)$ & $2105(2)$ & $2686(3)$ & $944(1)$ & $26(1)$ \\
$\mathrm{C}(15)$ & $1185(3)$ & $3619(3)$ & $637(2)$ & $32(1)$ \\
$\mathrm{C}(16)$ & $1425(3)$ & $4976(3)$ & $854(2)$ & $33(1)$ \\
$\mathrm{C}(17)$ & $1534(3)$ & $5106(3)$ & $1543(2)$ & $31(1)$ \\
$\mathrm{C}(18)$ & $2460(3)$ & $4190(3)$ & $1850(2)$ & $31(1)$ \\
$\mathrm{C}(19)$ & $2245(3)$ & $2830(3)$ & $1637(1)$ & $30(1)$ \\
$\mathrm{C}(20)$ & $1841(3)$ & $1287(3)$ & $736(2)$ & $32(1)$ \\
$\mathrm{C}(21)$ & $719(3)$ & $793(3)$ & $915(2)$ & $45(1)$ \\
$\mathrm{C}(22)$ & $1736(3)$ & $1183(4)$ & $44(2)$ & $51(1)$ \\
$\mathrm{C}(23)$ & $2824(3)$ & $415(3)$ & $1034(2)$ & $43(1)$ \\
$\mathrm{C}(24)$ & $364(3)$ & $4921(3)$ & $1736(2)$ & $34(1)$ \\
$\mathrm{C}(25)$ & $337(3)$ & $5176(4)$ & $2405(2)$ & $46(1)$ \\
$\mathrm{C}(26)$ & $1394(3)$ & $7422(3)$ & $1507(2)$ & $57(1)$ \\
$\mathrm{O}(5)$ & $4154(2)$ & $6483(3)$ & $2569(1)$ & $46(1)$ \\
& & & & \\
\hline
\end{tabular}

Table 3. Bond lengths $[\AA]$ and angles $\left[{ }^{\circ}\right]$ for $\mathbf{S - 2 9}$.

\begin{tabular}{ll}
\hline $\mathrm{O}(1)-\mathrm{C}(1)$ & $1.449(3)$ \\
$\mathrm{O}(2)-\mathrm{C}(13)$ & $1.435(4)$ \\
$\mathrm{O}(2)-\mathrm{C}(4)$ & $1.460(4)$ \\
$\mathrm{C}(1)-\mathrm{C}(6)$ & $1.529(4)$ \\
$\mathrm{C}(1)-\mathrm{C}(2)$ & $1.530(4)$ \\
$\mathrm{C}(1)-\mathrm{C}(7)$ & $1.571(4)$ \\
$\mathrm{C}(2)-\mathrm{C}(3)$ & $1.534(4)$ \\
$\mathrm{C}(3)-\mathrm{C}(4)$ & $1.527(4)$ \\
$\mathrm{C}(4)-\mathrm{C}(5)$ & $1.524(4)$ \\
$\mathrm{C}(4)-\mathrm{C}(11)$ & $1.535(4)$ \\
$\mathrm{C}(5)-\mathrm{C}(6)$ & $1.530(4)$ \\
$\mathrm{C}(7)-\mathrm{C}(9)$ & $1.535(4)$ \\
$\mathrm{C}(7)-\mathrm{C}(8)$ & $1.542(4)$
\end{tabular}




\begin{tabular}{|c|c|}
\hline$C(7)-C(10)$ & $1.543(4)$ \\
\hline$C(11)-C(12)$ & $1.529(4)$ \\
\hline $\mathrm{O}(3)-\mathrm{C}(14)$ & $1.436(3)$ \\
\hline $\mathrm{O}(4)-\mathrm{C}(26)$ & $1.426(4)$ \\
\hline $\mathrm{O}(4)-\mathrm{C}(17)$ & $1.456(4)$ \\
\hline$C(14)-C(19)$ & $1.529(4)$ \\
\hline$C(14)-C(15)$ & $1.535(4)$ \\
\hline$C(14)-C(20)$ & $1.569(4)$ \\
\hline$C(15)-C(16)$ & $1.528(4)$ \\
\hline$C(16)-C(17)$ & $1.525(5)$ \\
\hline$C(17)-C(18)$ & $1.528(4)$ \\
\hline$C(17)-C(24)$ & $1.532(4)$ \\
\hline$C(18)-C(19)$ & $1.525(4)$ \\
\hline$C(20)-C(22)$ & $1.529(5)$ \\
\hline$C(20)-C(23)$ & $1.538(4)$ \\
\hline$C(20)-C(21)$ & $1.544(4)$ \\
\hline$C(24)-C(25)$ & $1.522(5)$ \\
\hline $\mathrm{C}(13)-\mathrm{O}(2)-\mathrm{C}(4)$ & $117.0(2)$ \\
\hline $\mathrm{O}(1)-\mathrm{C}(1)-\mathrm{C}(6)$ & $104.7(2)$ \\
\hline $\mathrm{O}(1)-\mathrm{C}(1)-\mathrm{C}(2)$ & $108.5(2)$ \\
\hline$C(6)-C(1)-C(2)$ & $110.1(2)$ \\
\hline $\mathrm{O}(1)-\mathrm{C}(1)-\mathrm{C}(7)$ & $109.1(2)$ \\
\hline$C(6)-C(1)-C(7)$ & $112.1(2)$ \\
\hline$C(2)-C(1)-C(7)$ & $112.0(2)$ \\
\hline$C(1)-C(2)-C(3)$ & $113.6(2)$ \\
\hline$C(4)-C(3)-C(2)$ & $112.7(3)$ \\
\hline $\mathrm{O}(2)-\mathrm{C}(4)-\mathrm{C}(5)$ & $110.7(2)$ \\
\hline $\mathrm{O}(2)-\mathrm{C}(4)-\mathrm{C}(3)$ & $103.6(2)$ \\
\hline$C(5)-C(4)-C(3)$ & $107.7(3)$ \\
\hline $\mathrm{O}(2)-\mathrm{C}(4)-\mathrm{C}(11)$ & $109.5(2)$ \\
\hline$C(5)-C(4)-C(11)$ & $111.7(3)$ \\
\hline$C(3)-C(4)-C(11)$ & $113.3(2)$ \\
\hline$C(4)-C(5)-C(6)$ & $112.1(2)$ \\
\hline$C(1)-C(6)-C(5)$ & $113.5(3)$ \\
\hline $\mathrm{C}(9)-\mathrm{C}(7)-\mathrm{C}(8)$ & $107.3(3)$ \\
\hline$C(9)-C(7)-C(10)$ & $108.2(3)$ \\
\hline
\end{tabular}




$\begin{array}{ll}\mathrm{C}(8)-\mathrm{C}(7)-\mathrm{C}(10) & 107.4(3) \\ \mathrm{C}(9)-\mathrm{C}(7)-\mathrm{C}(1) & 111.2(3) \\ \mathrm{C}(8)-\mathrm{C}(7)-\mathrm{C}(1) & 110.6(2) \\ \mathrm{C}(10)-\mathrm{C}(7)-\mathrm{C}(1) & 111.8(3) \\ \mathrm{C}(12)-\mathrm{C}(11)-\mathrm{C}(4) & 114.7(3) \\ \mathrm{C}(26)-\mathrm{O}(4)-\mathrm{C}(17) & 117.3(2) \\ \mathrm{O}(3)-\mathrm{C}(14)-\mathrm{C}(19) & 110.6(2) \\ \mathrm{O}(3)-\mathrm{C}(14)-\mathrm{C}(15) & 103.5(2) \\ \mathrm{C}(19)-\mathrm{C}(14)-\mathrm{C}(15) & 109.0(3) \\ \mathrm{O}(3)-\mathrm{C}(14)-\mathrm{C}(20) & 108.5(2) \\ \mathrm{C}(19)-\mathrm{C}(14)-\mathrm{C}(20) & 111.9(3) \\ \mathrm{C}(15)-\mathrm{C}(14)-\mathrm{C}(20) & 113.0(2) \\ \mathrm{C}(16)-\mathrm{C}(15)-\mathrm{C}(14) & 112.9(3) \\ \mathrm{C}(17)-\mathrm{C}(16)-\mathrm{C}(15) & 112.4(3) \\ \mathrm{O}(4)-\mathrm{C}(17)-\mathrm{C}(16) & 111.0(3) \\ \mathrm{O}(4)-\mathrm{C}(17)-\mathrm{C}(18) & 102.5(2) \\ \mathrm{C}(16)-\mathrm{C}(17)-\mathrm{C}(18) & 108.4(3) \\ \mathrm{O}(4)-\mathrm{C}(17)-\mathrm{C}(24) & 110.1(3) \\ \mathrm{C}(16)-\mathrm{C}(17)-\mathrm{C}(24) & 110.8(3) \\ \mathrm{C}(18)-\mathrm{C}(17)-\mathrm{C}(24) & 113.7(3) \\ \mathrm{C}(19)-\mathrm{C}(18)-\mathrm{C}(17) & 113.3(3) \\ \mathrm{C}(18)-\mathrm{C}(19)-\mathrm{C}(14) & 113.0(3) \\ \mathrm{C}(22)-\mathrm{C}(20)-\mathrm{C}(23) & 108.0(3) \\ \mathrm{C}(22)-\mathrm{C}(20)-\mathrm{C}(21) & 108.7(3) \\ \mathrm{C}(23)-\mathrm{C}(20)-\mathrm{C}(21) & 107.5(3) \\ \mathrm{C}(22)-\mathrm{C}(20)-\mathrm{C}(14) & 110.0(3) \\ \mathrm{C}(23)-\mathrm{C}(20)-\mathrm{C}(14) & 110.3(3) \\ \mathrm{C}(21)-\mathrm{C}(20)-\mathrm{C}(14) & 112.2(3) \\ \mathrm{C}(25)-\mathrm{C}(24)-\mathrm{C}(17) & 115.6(3) \\ & \\ & \end{array}$

Table 4. Anisotropic displacement parameters $\left(\AA^{2} \times 10^{3}\right)$ for S-29. The anisotropic displacement factor exponent takes the form: $-2 \pi^{2}\left[h^{2} a^{* 2} U^{11}+\ldots+2 h \mathrm{ka}^{*} b^{*} U^{12}\right]$ 
Supporting Information: Glen Baghdasarian and K. A. Woerpel

\begin{tabular}{|c|c|c|c|c|c|c|}
\hline & $\mathrm{U}^{11}$ & $\mathrm{U}^{22}$ & $\mathrm{U}^{33}$ & $\mathrm{U}^{23}$ & $\mathrm{U}^{13}$ & $\mathrm{U}^{12}$ \\
\hline $\mathrm{O}(1)$ & $21(1)$ & $33(1)$ & $33(2)$ & $8(1)$ & $9(1)$ & $1(1)$ \\
\hline $\mathrm{O}(2)$ & $37(1)$ & $30(1)$ & $38(2)$ & $-3(1)$ & $9(1)$ & $8(1)$ \\
\hline$C(1)$ & $21(2)$ & $28(2)$ & $31(2)$ & $3(2)$ & $8(1)$ & $-3(1)$ \\
\hline $\mathrm{C}(2)$ & $23(2)$ & $30(2)$ & $29(2)$ & $6(2)$ & $5(1)$ & $3(1)$ \\
\hline$C(3)$ & $24(2)$ & $38(2)$ & $25(2)$ & $-2(2)$ & $4(1)$ & $-1(2)$ \\
\hline$C(4)$ & $29(2)$ & $26(2)$ & $30(2)$ & $-3(2)$ & $6(2)$ & $2(2)$ \\
\hline$C(5)$ & $25(2)$ & $29(2)$ & $31(2)$ & $4(2)$ & $8(2)$ & $1(2)$ \\
\hline$C(6)$ & $21(2)$ & $30(2)$ & $32(2)$ & $2(2)$ & $4(1)$ & $-2(2)$ \\
\hline$C(7)$ & $23(2)$ & $31(2)$ & $29(2)$ & $0(2)$ & $5(1)$ & $5(2)$ \\
\hline$C(8)$ & $37(2)$ & $34(2)$ & $51(2)$ & $-7(2)$ & $8(2)$ & $0(2)$ \\
\hline$C(9)$ & $44(2)$ & $37(2)$ & $45(2)$ & $3(2)$ & $12(2)$ & $15(2)$ \\
\hline$C(10)$ & $33(2)$ & $41(2)$ & $44(2)$ & $-1(2)$ & $16(2)$ & $2(2)$ \\
\hline $\mathrm{C}(11)$ & $28(2)$ & $32(2)$ & $35(2)$ & $1(2)$ & $3(2)$ & $-1(2)$ \\
\hline$C(12)$ & $37(2)$ & $34(2)$ & $49(2)$ & $-5(2)$ & $9(2)$ & $-2(2)$ \\
\hline$C(13)$ & $51(2)$ & $36(2)$ & $56(3)$ & $2(2)$ & $11(2)$ & $19(2)$ \\
\hline $\mathrm{O}(3)$ & $22(1)$ & $43(2)$ & $40(2)$ & $7(1)$ & $6(1)$ & $-5(1)$ \\
\hline $\mathrm{O}(4)$ & $34(1)$ & $24(1)$ & $59(2)$ & $-1(1)$ & $3(1)$ & $-3(1)$ \\
\hline$C(14)$ & $20(2)$ & $28(2)$ & $30(2)$ & $2(2)$ & $5(1)$ & $-3(1)$ \\
\hline$C(15)$ & $24(2)$ & $37(2)$ & $35(2)$ & $1(2)$ & $3(2)$ & $0(2)$ \\
\hline$C(16)$ & $24(2)$ & $33(2)$ & $41(2)$ & $10(2)$ & $2(2)$ & $0(2)$ \\
\hline$C(17)$ & $25(2)$ & $23(2)$ & $44(2)$ & $1(2)$ & $3(2)$ & $-6(2)$ \\
\hline$C(18)$ & $27(2)$ & $32(2)$ & $33(2)$ & $0(2)$ & $4(2)$ & $-2(2)$ \\
\hline C(19) & $23(2)$ & $29(2)$ & $38(2)$ & $3(2)$ & $3(2)$ & $0(2)$ \\
\hline$C(20)$ & $25(2)$ & $31(2)$ & $39(2)$ & $-3(2)$ & $7(2)$ & $-5(2)$ \\
\hline $\mathrm{C}(21)$ & $31(2)$ & $35(2)$ & $69(3)$ & $-5(2)$ & $14(2)$ & $-6(2)$ \\
\hline$C(22)$ & $59(3)$ & $50(2)$ & $46(3)$ & $-9(2)$ & $10(2)$ & $-7(2)$ \\
\hline$C(23)$ & $31(2)$ & $34(2)$ & $65(3)$ & $-3(2)$ & $14(2)$ & $1(2)$ \\
\hline $\mathrm{C}(24)$ & $29(2)$ & $32(2)$ & $40(2)$ & $0(2)$ & $5(2)$ & $1(2)$ \\
\hline$C(25)$ & $31(2)$ & $56(3)$ & $52(3)$ & $1(2)$ & $10(2)$ & $6(2)$ \\
\hline$C(26)$ & $48(2)$ & $28(2)$ & $91(3)$ & $6(2)$ & $2(2)$ & $-1(2)$ \\
\hline $\mathrm{O}(5)$ & $34(2)$ & $63(2)$ & $42(2)$ & $-19(1)$ & $11(1)$ & $-11(1)$ \\
\hline
\end{tabular}


Table 5. Hydrogen coordinates ( $\left.\times 10^{4}\right)$ and isotropic displacement parameters $\left(\AA^{2} \times 10^{3}\right)$ for $\mathbf{S - 2 9}$.

\begin{tabular}{|c|c|c|c|c|}
\hline & $\mathrm{x}$ & $\mathrm{y}$ & z & $\mathrm{U}(\mathrm{eq})$ \\
\hline $\mathrm{H}(1)$ & $5600(30)$ & $2510(30)$ & 1797(17) & $58(12)$ \\
\hline $\mathrm{H}(2 \mathrm{~A})$ & 7812 & 4276 & 1454 & 33 \\
\hline $\mathrm{H}(2 \mathrm{~B})$ & 7408 & 3394 & 1958 & 33 \\
\hline $\mathrm{H}(3 \mathrm{~A})$ & 6099 & 4920 & 2164 & 35 \\
\hline $\mathrm{H}(3 \mathrm{~B})$ & 7336 & 5570 & 2221 & 35 \\
\hline $\mathrm{H}(5 \mathrm{~A})$ & 4887 & 6174 & 698 & 34 \\
\hline $\mathrm{H}(5 \mathrm{~B})$ & 4610 & 5274 & 1230 & 34 \\
\hline $\mathrm{H}(6 \mathrm{~A})$ & 5001 & 4024 & 420 & 33 \\
\hline $\mathrm{H}(6 \mathrm{~B})$ & 6214 & 4709 & 443 & 33 \\
\hline $\mathrm{H}(8 \mathrm{~A})$ & 6143 & 859 & 207 & 61 \\
\hline $\mathrm{H}(8 \mathrm{~B})$ & 5353 & 2094 & 103 & 61 \\
\hline $\mathrm{H}(8 \mathrm{C})$ & 5295 & 1185 & 670 & 61 \\
\hline $\mathrm{H}(9 \mathrm{~A})$ & 8087 & 1858 & 1523 & 62 \\
\hline $\mathrm{H}(9 \mathrm{~B})$ & 7786 & 730 & 1043 & 62 \\
\hline $\mathrm{H}(9 \mathrm{C})$ & 6957 & 1023 & 1520 & 62 \\
\hline $\mathrm{H}(10 \mathrm{~A})$ & 8187 & 3303 & 622 & 58 \\
\hline $\mathrm{H}(10 \mathrm{~B})$ & 7116 & 3353 & 68 & 58 \\
\hline $\mathrm{H}(10 \mathrm{C})$ & 7881 & 2102 & 188 & 58 \\
\hline $\mathrm{H}(11 \mathrm{~A})$ & 7493 & 6129 & 956 & 38 \\
\hline $\mathrm{H}(11 \mathrm{~B})$ & 6672 & 7326 & 799 & 38 \\
\hline $\mathrm{H}(12 \mathrm{~A})$ & 8498 & 7984 & 1304 & 60 \\
\hline $\mathrm{H}(12 \mathrm{~B})$ & 8395 & 7105 & 1875 & 60 \\
\hline $\mathrm{H}(12 \mathrm{C})$ & 7568 & 8303 & 1722 & 60 \\
\hline $\mathrm{H}(13 \mathrm{~A})$ & 4843 & 8737 & 1794 & 71 \\
\hline $\mathrm{H}(13 \mathrm{~B})$ & 4455 & 7857 & 1209 & 71 \\
\hline $\mathrm{H}(13 \mathrm{C})$ & 5638 & 8628 & 1286 & 71 \\
\hline
\end{tabular}


Supporting Information: Glen Baghdasarian and K. A. Woerpel

\begin{tabular}{|c|c|c|c|c|}
\hline $\mathrm{H}(3)$ & $3740(30)$ & $2930(30)$ & 989(16) & $56(13)$ \\
\hline $\mathrm{H}(15 \mathrm{~A})$ & 1147 & 3585 & 190 & 39 \\
\hline $\mathrm{H}(15 \mathrm{~B})$ & 426 & 3356 & 722 & 39 \\
\hline $\mathrm{H}(16 \mathrm{~A})$ & 2147 & 5268 & 733 & 40 \\
\hline $\mathrm{H}(16 \mathrm{~B})$ & 792 & 5525 & 650 & 40 \\
\hline $\mathrm{H}(18 \mathrm{~A})$ & 3216 & 4465 & 1765 & 37 \\
\hline $\mathrm{H}(18 \mathrm{~B})$ & 2498 & 4225 & 2297 & 37 \\
\hline $\mathrm{H}(19 \mathrm{~A})$ & 1540 & 2517 & 1770 & 36 \\
\hline $\mathrm{H}(19 \mathrm{~B})$ & 2897 & 2300 & 1834 & 36 \\
\hline $\mathrm{H}(21 \mathrm{~A})$ & 74 & 1332 & 733 & 67 \\
\hline $\mathrm{H}(21 \mathrm{~B})$ & 794 & 806 & 1360 & 67 \\
\hline $\mathrm{H}(21 \mathrm{C})$ & 576 & -73 & 767 & 67 \\
\hline $\mathrm{H}(22 \mathrm{~A})$ & 2440 & 1505 & -76 & 77 \\
\hline $\mathrm{H}(22 \mathrm{~B})$ & 1075 & 1681 & -157 & 77 \\
\hline $\mathrm{H}(22 \mathrm{C})$ & 1626 & 298 & -79 & 77 \\
\hline $\mathrm{H}(23 \mathrm{~A})$ & 3551 & 706 & 929 & 64 \\
\hline $\mathrm{H}(23 \mathrm{~B})$ & 2667 & -449 & 884 & 64 \\
\hline $\mathrm{H}(23 \mathrm{C})$ & 2881 & 431 & 1478 & 64 \\
\hline $\mathrm{H}(24 \mathrm{~A})$ & -202 & 5483 & 1485 & 40 \\
\hline $\mathrm{H}(24 \mathrm{~B})$ & 110 & 4042 & 1642 & 40 \\
\hline $\mathrm{H}(25 \mathrm{~A})$ & -444 & 5039 & 2482 & 69 \\
\hline $\mathrm{H}(25 \mathrm{~B})$ & 567 & 6051 & 2503 & 69 \\
\hline $\mathrm{H}(25 \mathrm{C})$ & 871 & 4604 & 2660 & 69 \\
\hline $\mathrm{H}(26 \mathrm{~A})$ & 1802 & 8179 & 1682 & 85 \\
\hline $\mathrm{H}(26 \mathrm{~B})$ & 621 & 7406 & 1608 & 85 \\
\hline $\mathrm{H}(26 \mathrm{C})$ & 1334 & 7432 & 1063 & 85 \\
\hline $\mathrm{H}(51)$ & $4650(40)$ & $6690(40)$ & $2280(20)$ & $97(17)$ \\
\hline $\mathrm{H}(52)$ & $3510(40)$ & $6520(50)$ & $2300(20)$ & $104(19)$ \\
\hline
\end{tabular}

Table 6. Torsion angles $\left[^{\circ}\right]$ for $\mathbf{S - 2 9}$.

$\mathrm{O}(1)-\mathrm{C}(1)-\mathrm{C}(2)-\mathrm{C}(3)$




$\begin{array}{lc}\mathrm{C}(6)-\mathrm{C}(1)-\mathrm{C}(2)-\mathrm{C}(3) & 48.8(3) \\ \mathrm{C}(7)-\mathrm{C}(1)-\mathrm{C}(2)-\mathrm{C}(3) & 174.2(2) \\ \mathrm{C}(1)-\mathrm{C}(2)-\mathrm{C}(3)-\mathrm{C}(4) & -54.5(3) \\ \mathrm{C}(13)-\mathrm{O}(2)-\mathrm{C}(4)-\mathrm{C}(5) & -61.9(3) \\ \mathrm{C}(13)-\mathrm{O}(2)-\mathrm{C}(4)-\mathrm{C}(3) & -177.1(3) \\ \mathrm{C}(13)-\mathrm{O}(2)-\mathrm{C}(4)-\mathrm{C}(11) & 61.7(3) \\ \mathrm{C}(2)-\mathrm{C}(3)-\mathrm{C}(4)-\mathrm{O}(2) & 174.4(2) \\ \mathrm{C}(2)-\mathrm{C}(3)-\mathrm{C}(4)-\mathrm{C}(5) & 57.0(3) \\ \mathrm{C}(2)-\mathrm{C}(3)-\mathrm{C}(4)-\mathrm{C}(11) & -67.0(3) \\ \mathrm{O}(2)-\mathrm{C}(4)-\mathrm{C}(5)-\mathrm{C}(6) & -170.7(2) \\ \mathrm{C}(3)-\mathrm{C}(4)-\mathrm{C}(5)-\mathrm{C}(6) & -58.0(3) \\ \mathrm{C}(11)-\mathrm{C}(4)-\mathrm{C}(5)-\mathrm{C}(6) & 67.1(3) \\ \mathrm{O}(1)-\mathrm{C}(1)-\mathrm{C}(6)-\mathrm{C}(5) & 66.5(3) \\ \mathrm{C}(2)-\mathrm{C}(1)-\mathrm{C}(6)-\mathrm{C}(5) & -49.9(3) \\ \mathrm{C}(7)-\mathrm{C}(1)-\mathrm{C}(6)-\mathrm{C}(5) & -175.3(2) \\ \mathrm{C}(4)-\mathrm{C}(5)-\mathrm{C}(6)-\mathrm{C}(1) & 56.7(3) \\ \mathrm{O}(1)-\mathrm{C}(1)-\mathrm{C}(7)-\mathrm{C}(9) & -71.0(3) \\ \mathrm{C}(6)-\mathrm{C}(1)-\mathrm{C}(7)-\mathrm{C}(9) & 173.5(3) \\ \mathrm{C}(2)-\mathrm{C}(1)-\mathrm{C}(7)-\mathrm{C}(9) & 49.1(3) \\ \mathrm{O}(1)-\mathrm{C}(1)-\mathrm{C}(7)-\mathrm{C}(8) & 48.1(3) \\ \mathrm{C}(6)-\mathrm{C}(1)-\mathrm{C}(7)-\mathrm{C}(8) & -67.3(3) \\ \mathrm{C}(2)-\mathrm{C}(1)-\mathrm{C}(7)-\mathrm{C}(8) & 168.3(3) \\ \mathrm{O}(1)-\mathrm{C}(1)-\mathrm{C}(7)-\mathrm{C}(10) & 167.8(2) \\ \mathrm{C}(6)-\mathrm{C}(1)-\mathrm{C}(7)-\mathrm{C}(10) & 52.4(3) \\ \mathrm{C}(2)-\mathrm{C}(1)-\mathrm{C}(7)-\mathrm{C}(10) & -72.0(3) \\ \mathrm{O}(2)-\mathrm{C}(4)-\mathrm{C}(11)-\mathrm{C}(12) & 48.0(3) \\ \mathrm{C}(5)-\mathrm{C}(4)-\mathrm{C}(11)-\mathrm{C}(12) & 171.0(3) \\ \mathrm{C}(3)-\mathrm{C}(4)-\mathrm{C}(11)-\mathrm{C}(12) & -67.1(4) \\ \mathrm{O}(3)-\mathrm{C}(14)-\mathrm{C}(15)-\mathrm{C}(16) & -64.5(3) \\ \mathrm{C}(19)-\mathrm{C}(14)-\mathrm{C}(15)-\mathrm{C}(16) & 53.3(3) \\ \mathrm{C}(20)-\mathrm{C}(14)-\mathrm{C}(15)-\mathrm{C}(16) & 178.3(3) \\ \mathrm{C}(14)-\mathrm{C}(15)-\mathrm{C}(16)-\mathrm{C}(17) & -57.0(4) \\ \mathrm{C}(26)-\mathrm{O}(4)-\mathrm{C}(17)-\mathrm{C}(16) & 59.7(4) \\ \mathrm{C}(26)-\mathrm{O}(4)-\mathrm{C}(17)-\mathrm{C}(18) & 175.2(3) \\ \mathrm{C}(26)-\mathrm{O}(4)-\mathrm{C}(17)-\mathrm{C}(24) & -63.4(4) \\ \mathrm{C}(15)-\mathrm{C}(16)-\mathrm{C}(17)-\mathrm{O}(4) & 167.0(2) \\ & \\ & \\ & \end{array}$




$\begin{array}{lc}\mathrm{C}(15)-\mathrm{C}(16)-\mathrm{C}(17)-\mathrm{C}(18) & 55.2(3) \\ \mathrm{C}(15)-\mathrm{C}(16)-\mathrm{C}(17)-\mathrm{C}(24) & -70.3(3) \\ \mathrm{O}(4)-\mathrm{C}(17)-\mathrm{C}(18)-\mathrm{C}(19) & -172.1(3) \\ \mathrm{C}(16)-\mathrm{C}(17)-\mathrm{C}(18)-\mathrm{C}(19) & -54.7(3) \\ \mathrm{C}(24)-\mathrm{C}(17)-\mathrm{C}(18)-\mathrm{C}(19) & 69.0(4) \\ \mathrm{C}(17)-\mathrm{C}(18)-\mathrm{C}(19)-\mathrm{C}(14) & 55.4(4) \\ \mathrm{O}(3)-\mathrm{C}(14)-\mathrm{C}(19)-\mathrm{C}(18) & 60.9(3) \\ \mathrm{C}(15)-\mathrm{C}(14)-\mathrm{C}(19)-\mathrm{C}(18) & -52.3(3) \\ \mathrm{C}(20)-\mathrm{C}(14)-\mathrm{C}(19)-\mathrm{C}(18) & -178.0(2) \\ \mathrm{O}(3)-\mathrm{C}(14)-\mathrm{C}(20)-\mathrm{C}(22) & -56.3(3) \\ \mathrm{C}(19)-\mathrm{C}(14)-\mathrm{C}(20)-\mathrm{C}(22) & -178.7(3) \\ \mathrm{C}(15)-\mathrm{C}(14)-\mathrm{C}(20)-\mathrm{C}(22) & 57.9(3) \\ \mathrm{O}(3)-\mathrm{C}(14)-\mathrm{C}(20)-\mathrm{C}(23) & 62.7(3) \\ \mathrm{C}(19)-\mathrm{C}(14)-\mathrm{C}(20)-\mathrm{C}(23) & -59.6(3) \\ \mathrm{C}(15)-\mathrm{C}(14)-\mathrm{C}(20)-\mathrm{C}(23) & 177.0(3) \\ \mathrm{O}(3)-\mathrm{C}(14)-\mathrm{C}(20)-\mathrm{C}(21) & -177.5(3) \\ \mathrm{C}(19)-\mathrm{C}(14)-\mathrm{C}(20)-\mathrm{C}(21) & 60.2(3) \\ \mathrm{C}(15)-\mathrm{C}(14)-\mathrm{C}(20)-\mathrm{C}(21) & -63.3(4) \\ \mathrm{O}(4)-\mathrm{C}(17)-\mathrm{C}(24)-\mathrm{C}(25) & -50.1(4) \\ \mathrm{C}(16)-\mathrm{C}(17)-\mathrm{C}(24)-\mathrm{C}(25) & -173.3(3) \\ \mathrm{C}(18)-\mathrm{C}(17)-\mathrm{C}(24)-\mathrm{C}(25) & 64.3(4) \\ & \end{array}$

1. SMART Software Users Guide, Version 5.1, Bruker Analytical X-Ray Systems, Inc.; Madison, WI 1999.

2. SAINT Software Users Guide, Version 6.0, Bruker Analytical X-Ray Systems, Inc.; Madison, WI 1999.

3. Sheldrick, G. M. SADABS, Version 2.10, Bruker Analytical X-Ray Systems, Inc.; Madison, WI 2002.

4. Sheldrick, G. M. SHELXTL Version 6.12, Bruker Analytical X-Ray Systems, Inc.; Madison, WI 2001.

5. International Tables for X-Ray Crystallography 1992, Vol. C., Dordrecht: Kluwer Academic Publishers 


\section{Proofs of Diastereoselectivity}
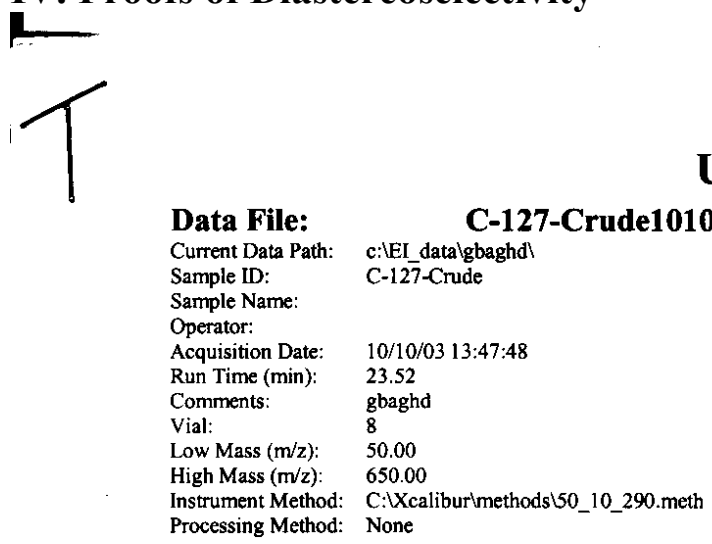

Acquisition Date: $\quad$ 10/10/03 13:47:48

EI_datalgbagh

.00
.00

UCI MS Facility

RT: $0.00-26.03$ SM: $3 G$

\section{C-127-Crude10100009_134734}

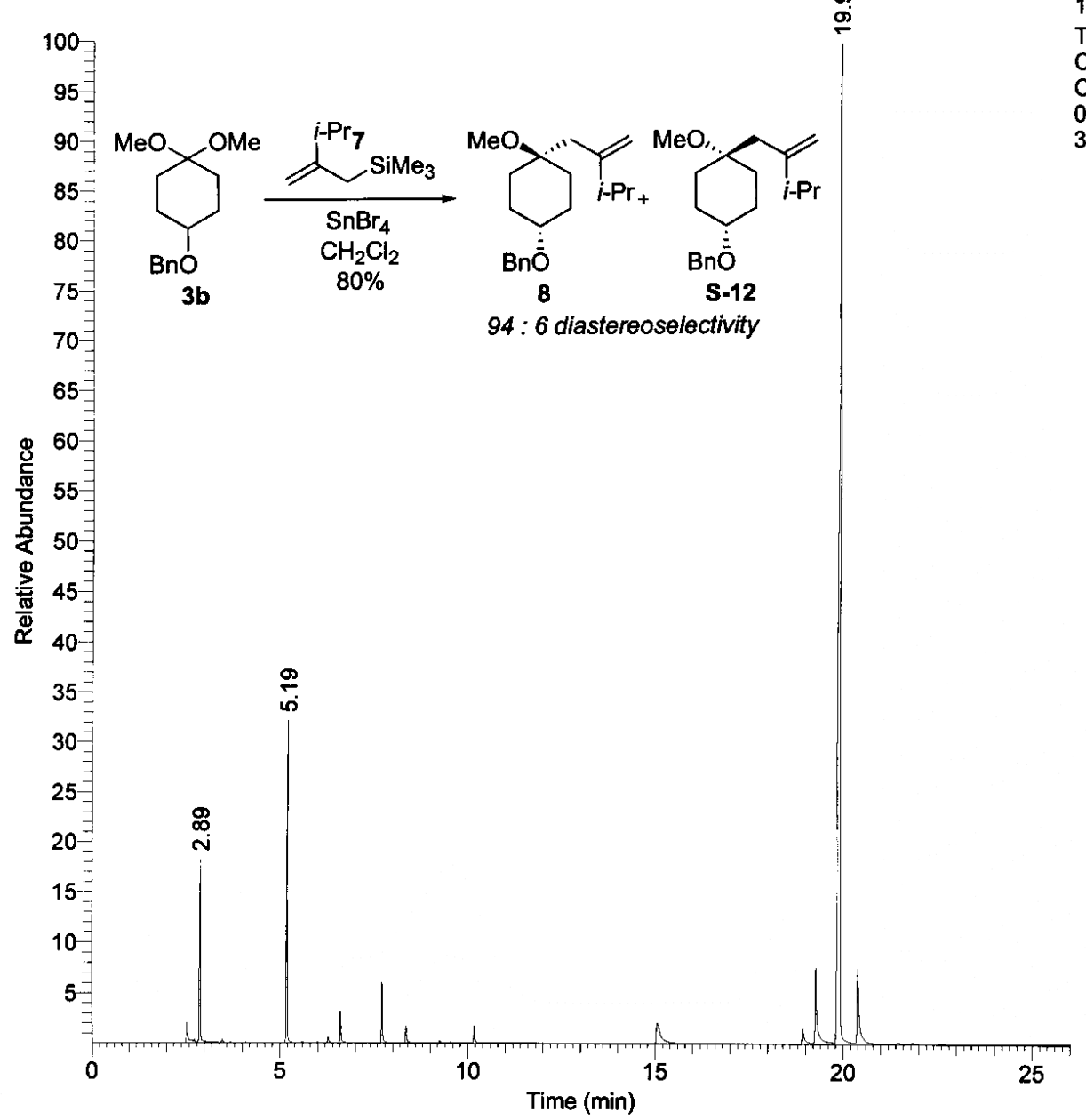

NL: 1.87E9

TIC MS

C-127-

Crude1010

00091347

Page 1 of 4 


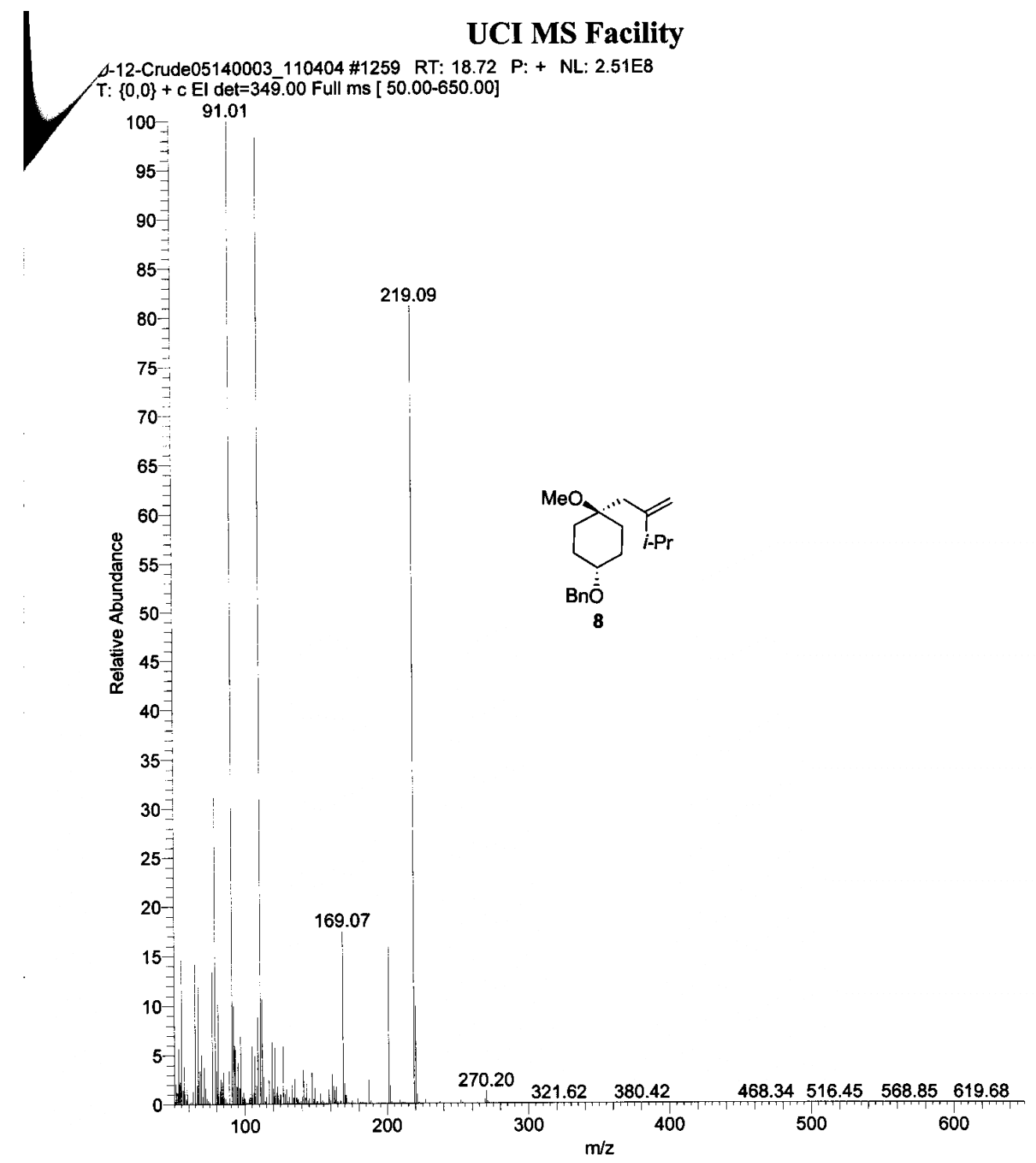

Page 3 of 3 


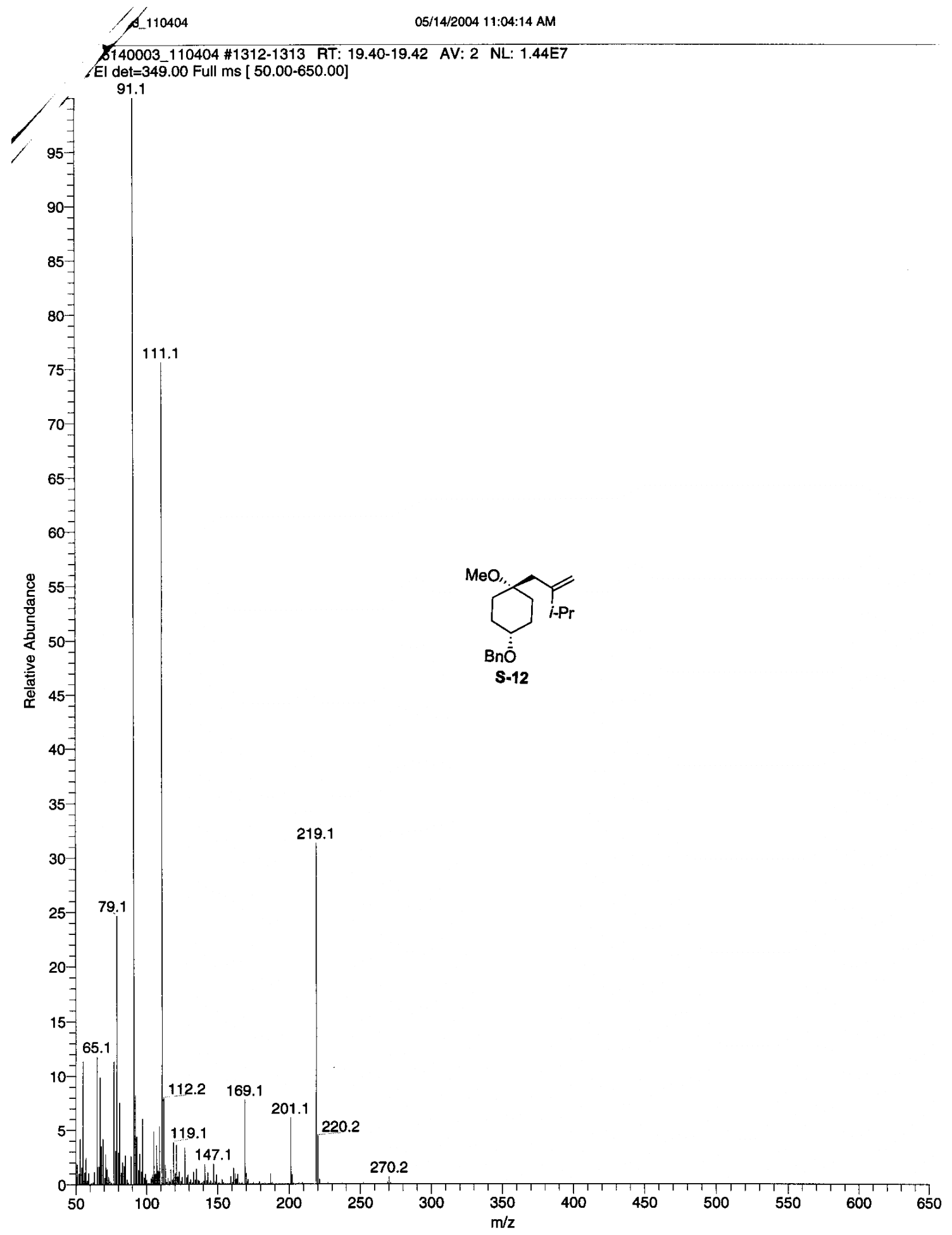




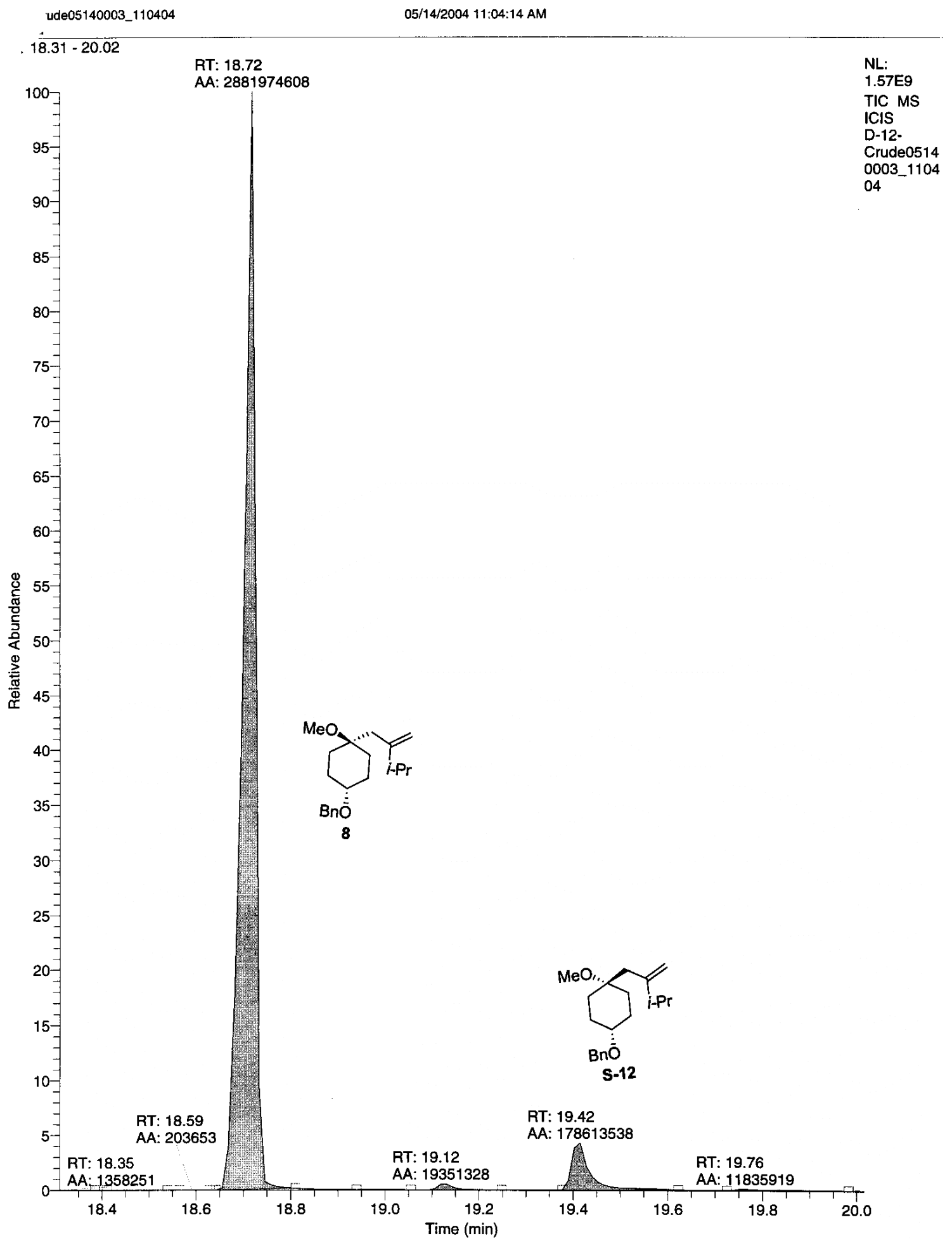



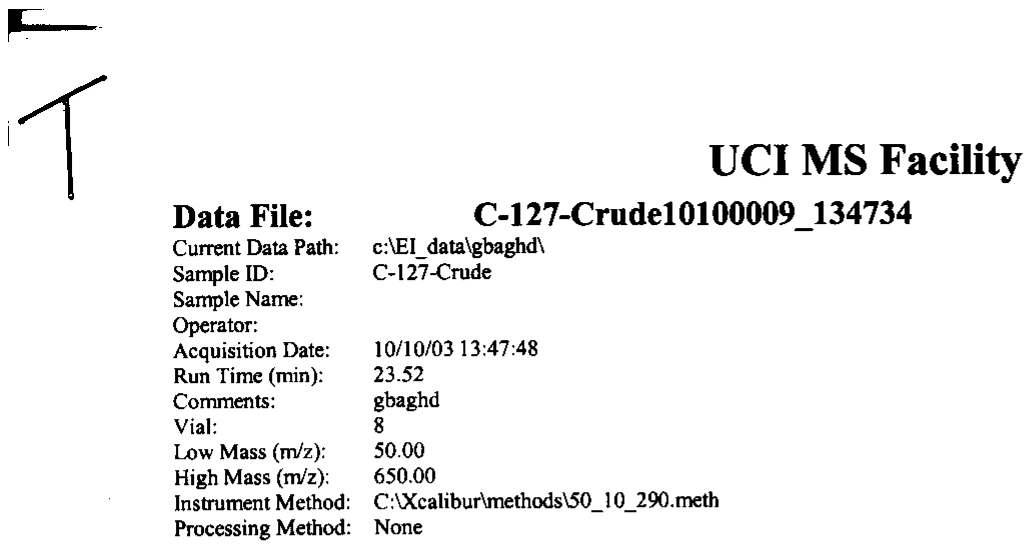

RT: $0.00-26.03$ SM: $3 G$

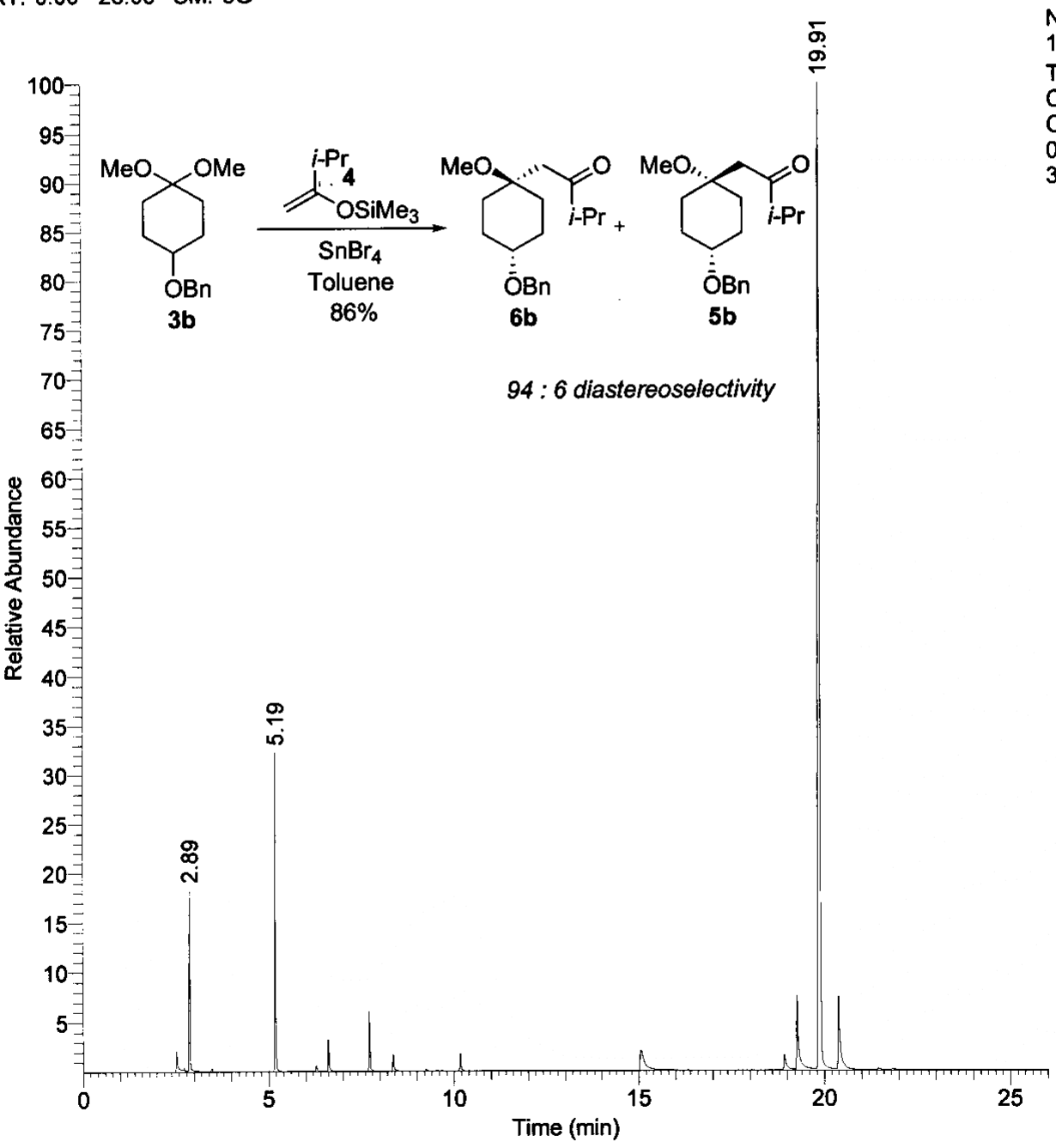




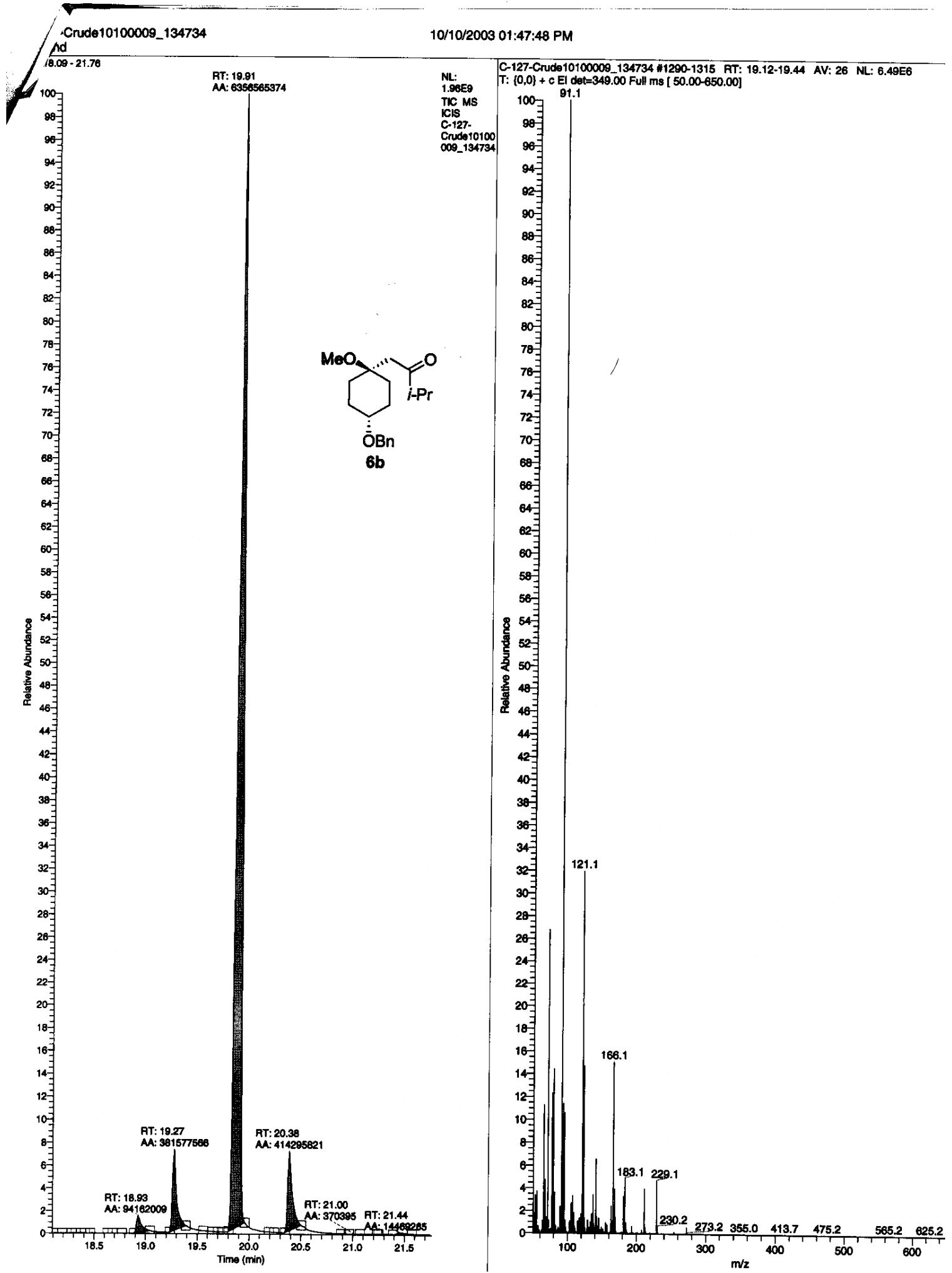




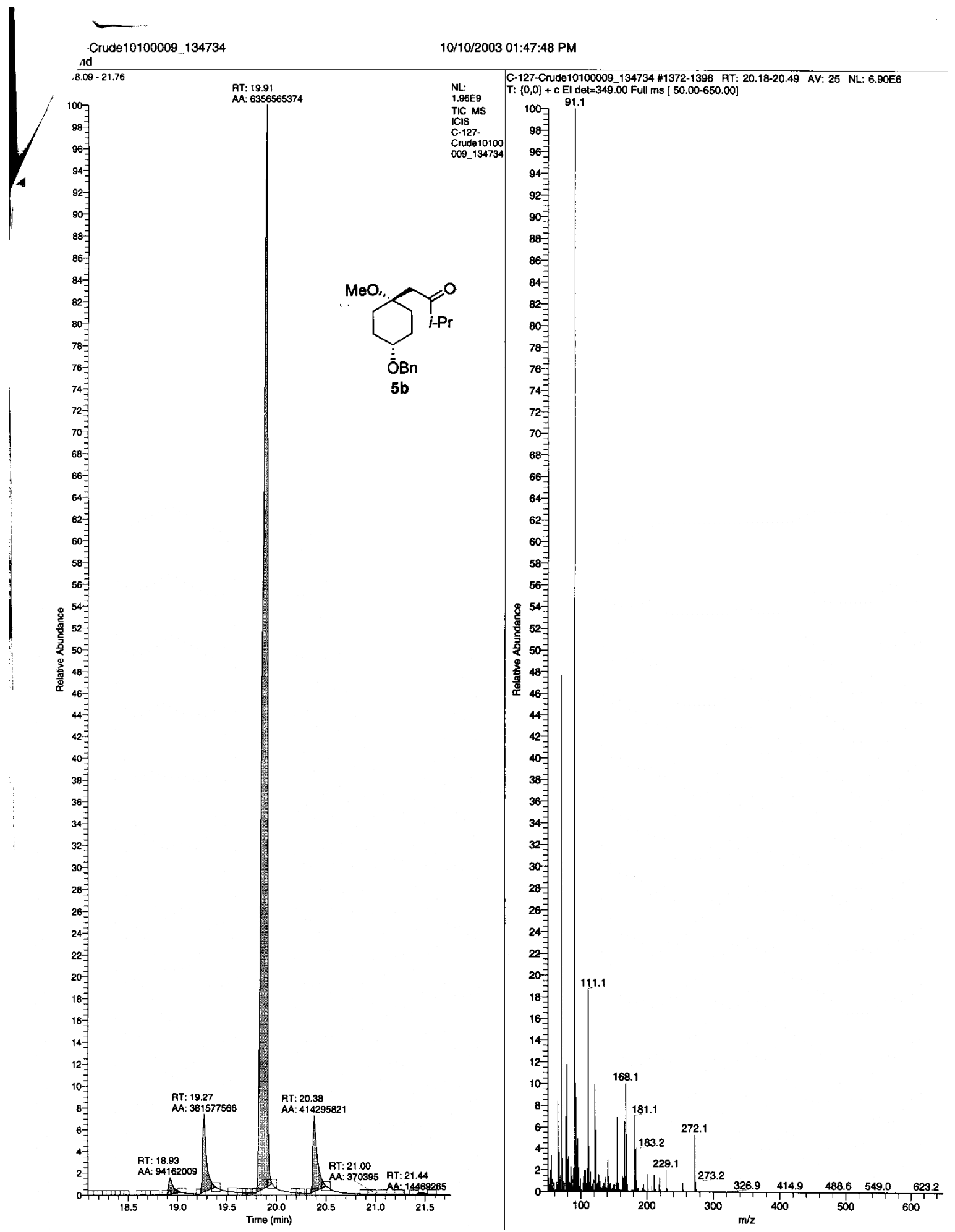




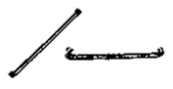

UCI MS Facility

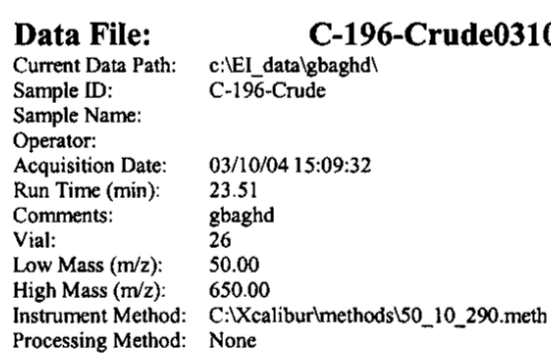

RT: $0.00-26.01 S M: 3 G$

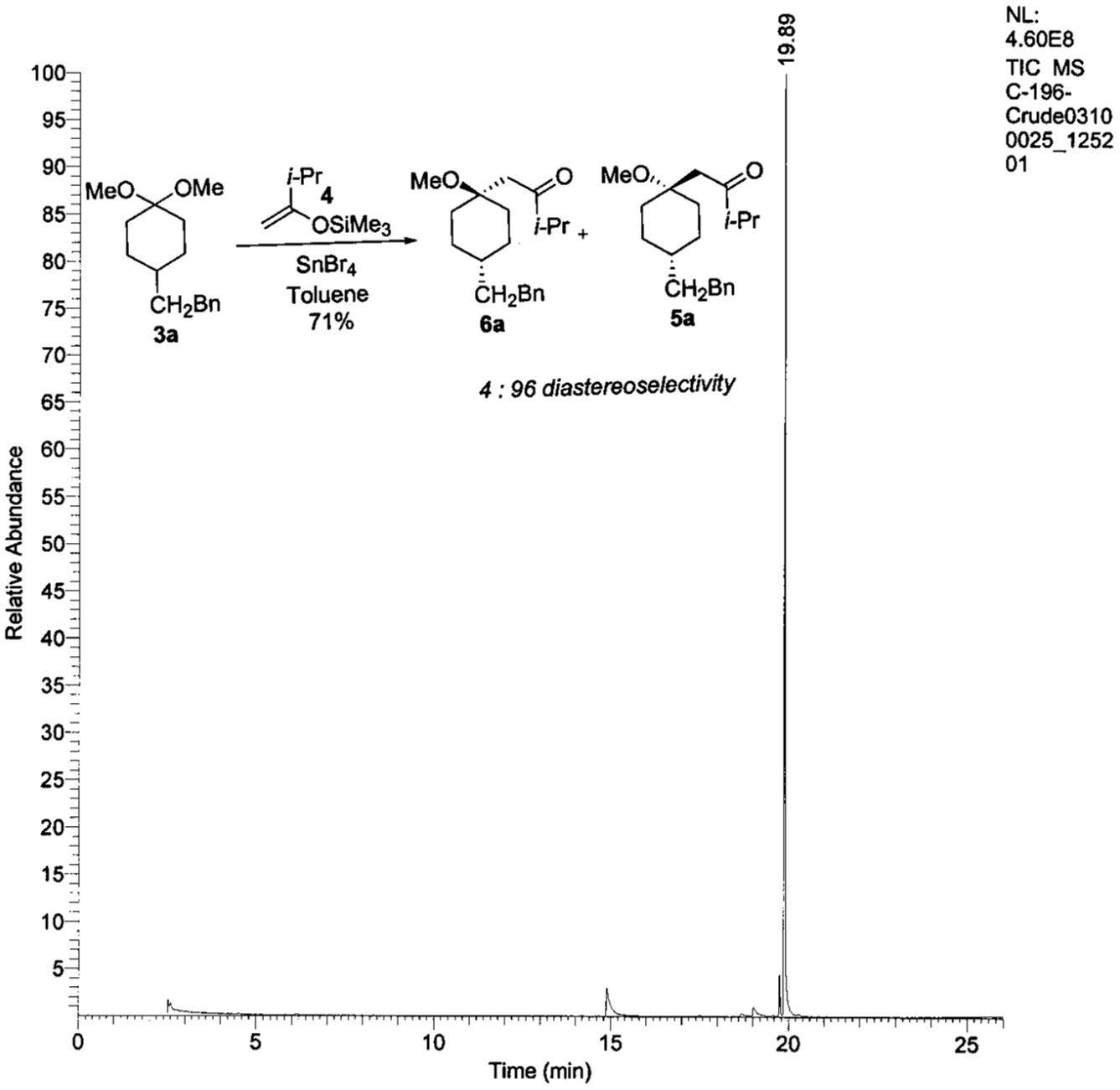

Page 1 of 2 


\section{UCI MS Facility}

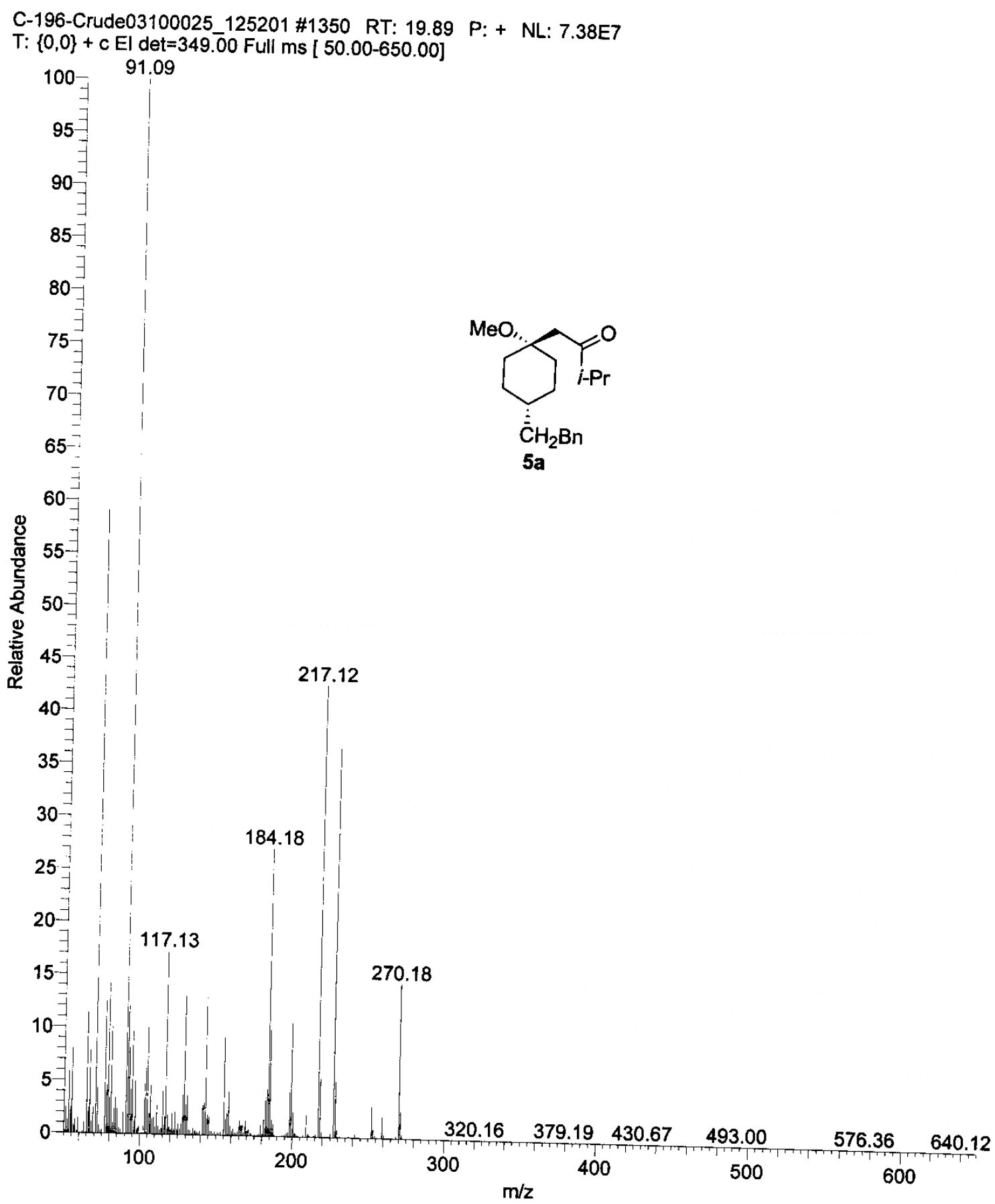




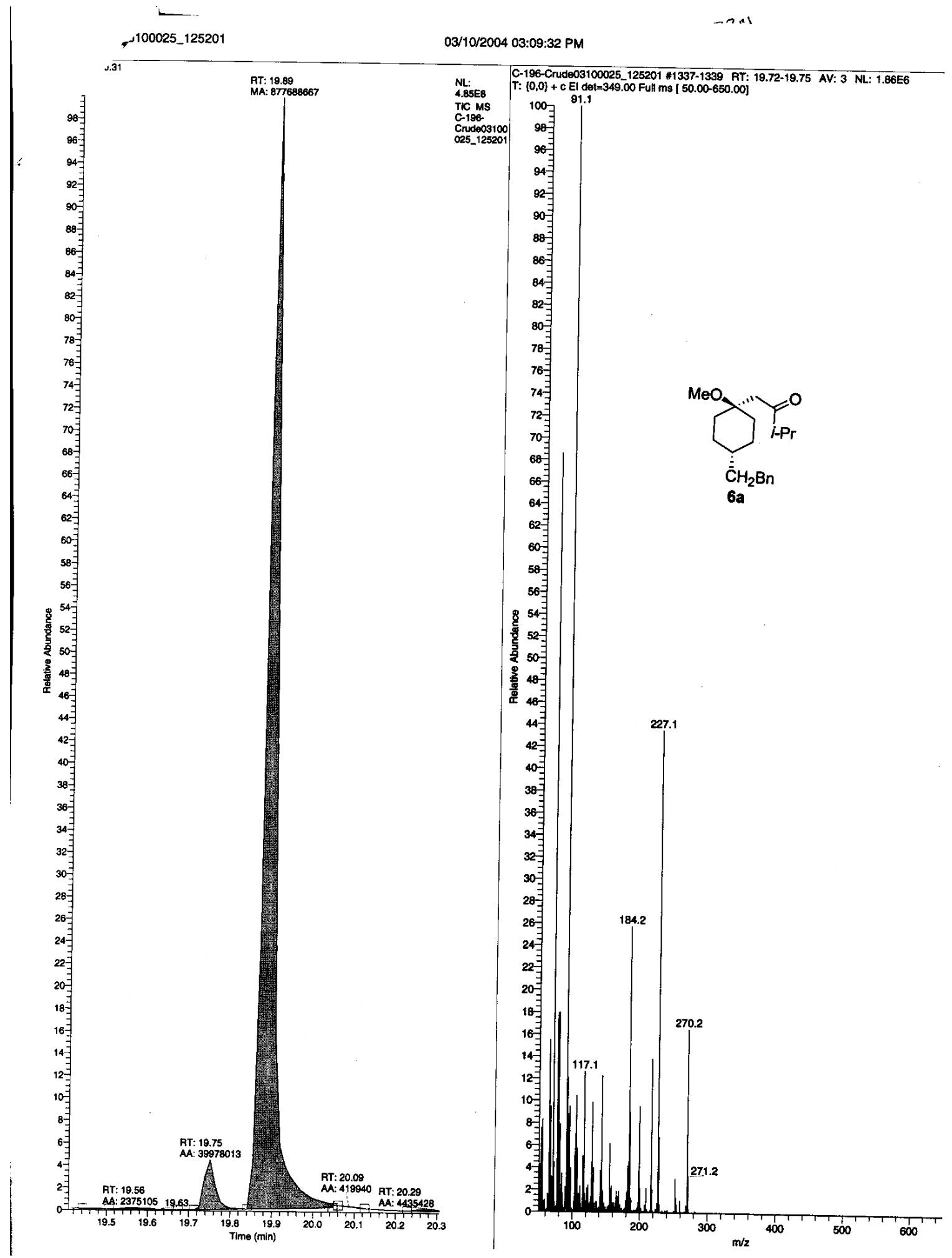




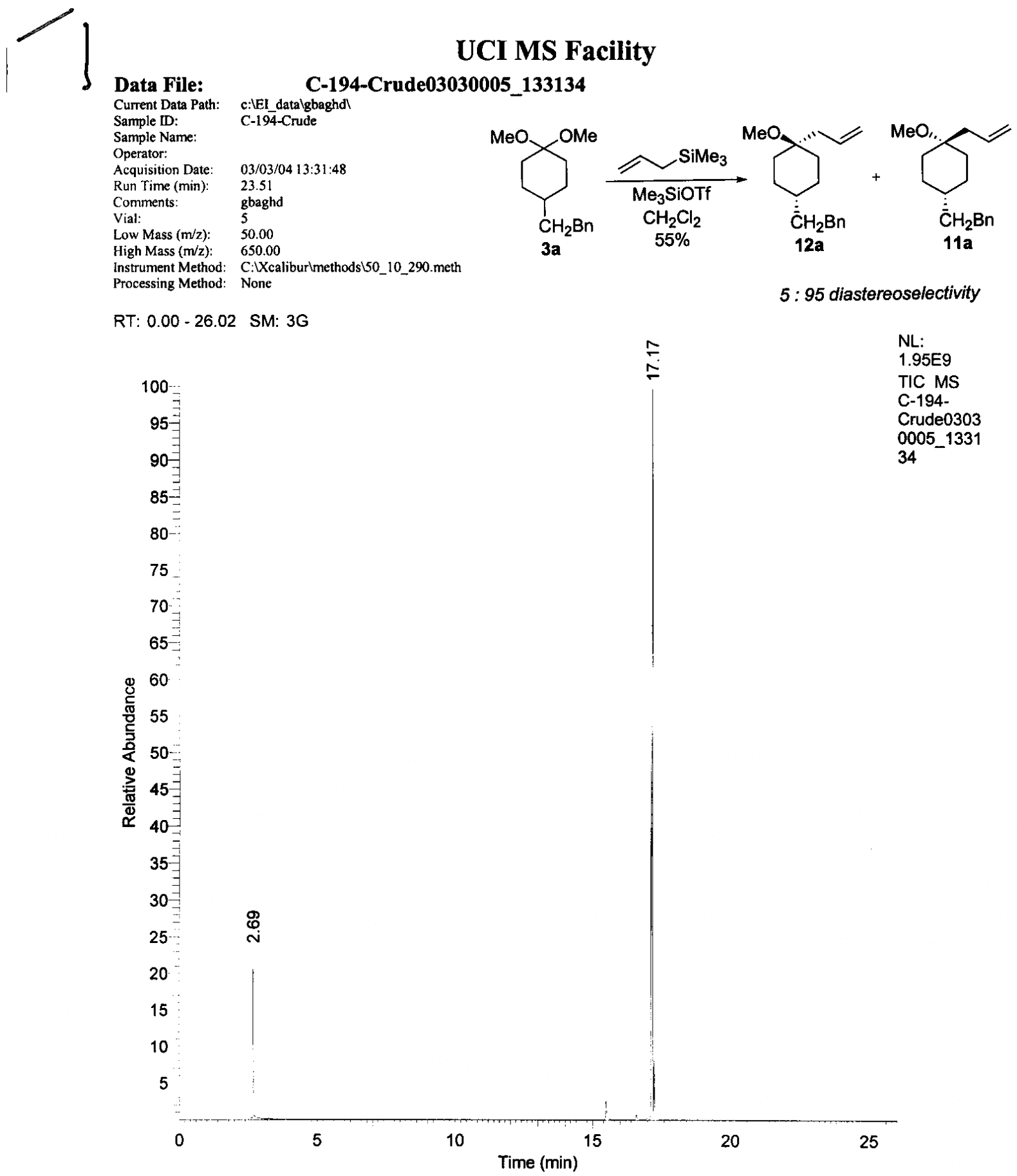

Page 1 of 3 


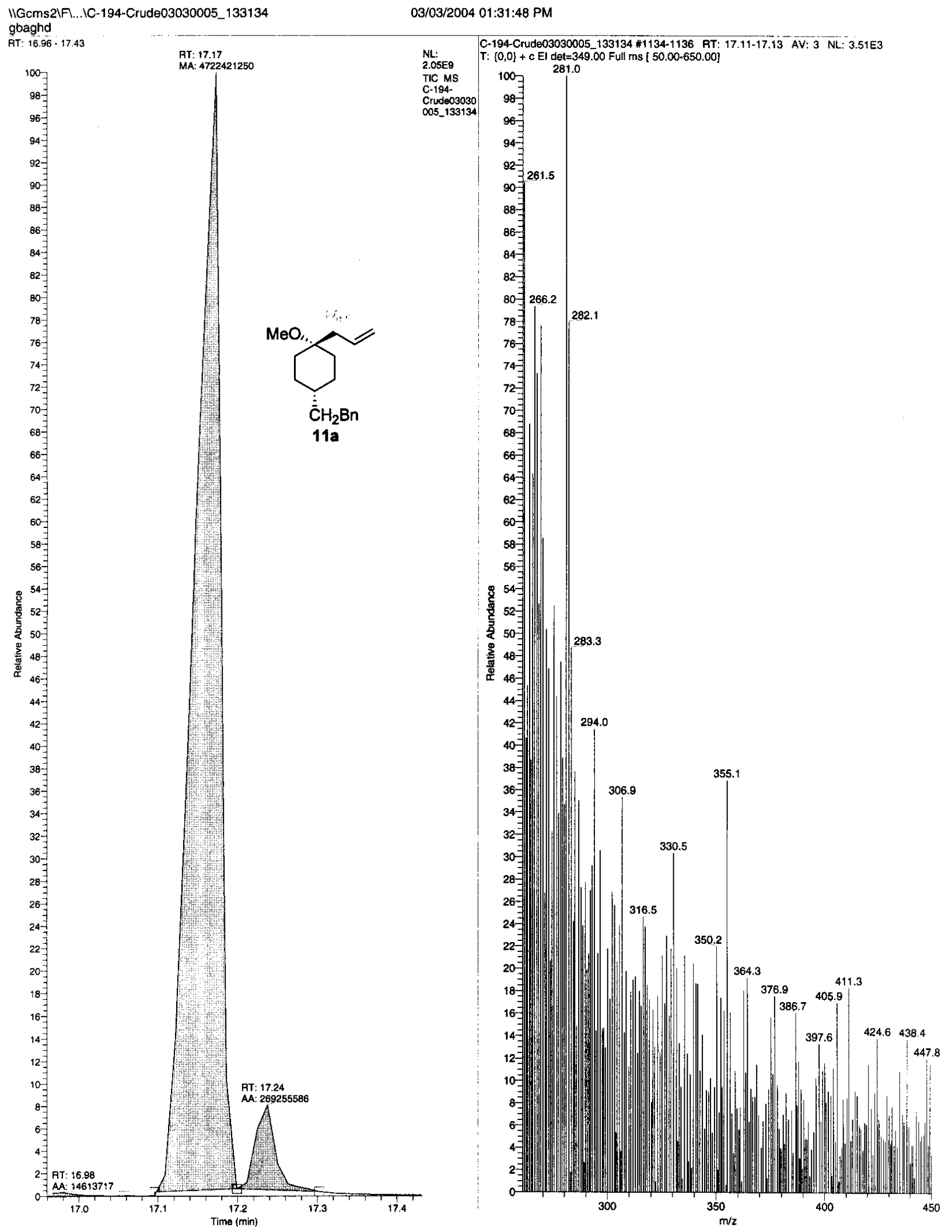




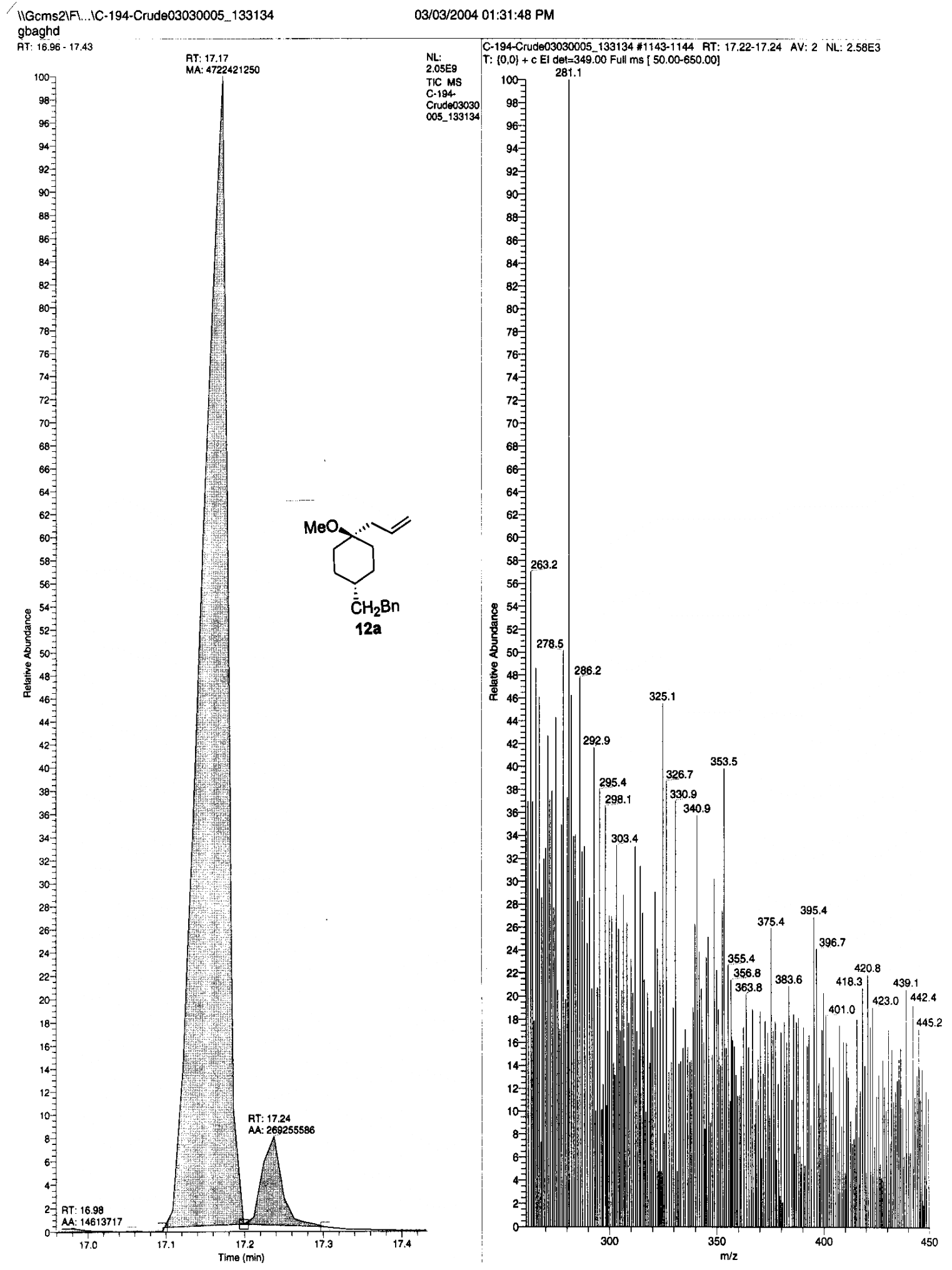




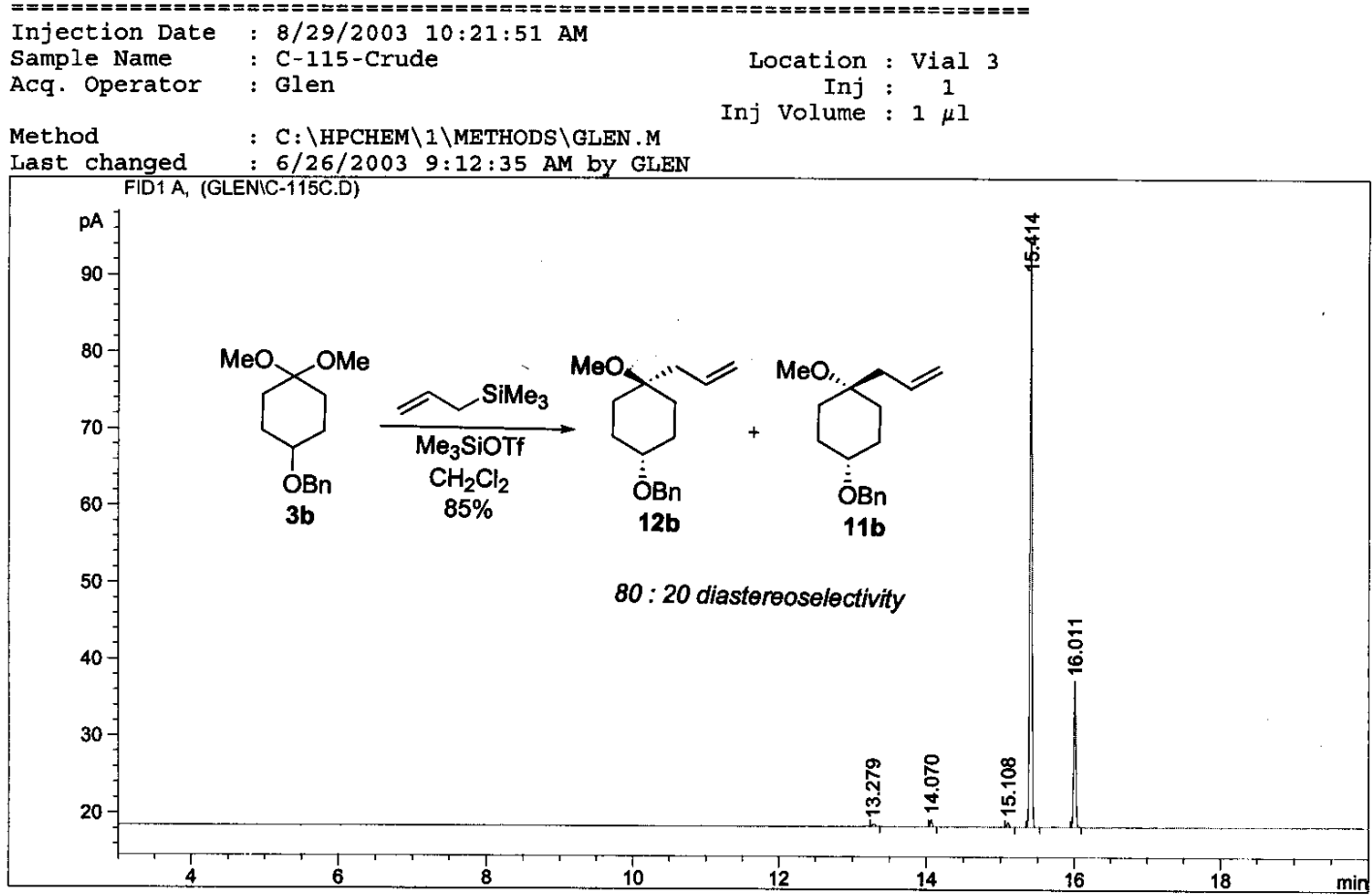

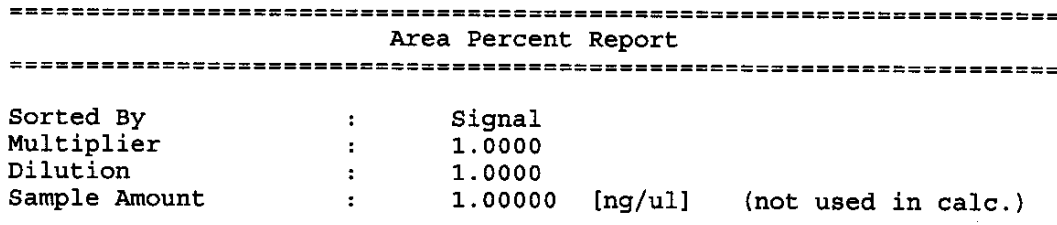

Signal 1: FID1 A,

\begin{tabular}{|c|c|c|c|c|c|c|}
\hline $\begin{array}{l}\text { eak } \\
\#\end{array}$ & $\begin{array}{l}\text { RetTime } \\
\text { [min] }\end{array}$ & Type & $\begin{array}{l}\text { Width } \\
\text { [min] }\end{array}$ & $\begin{array}{r}\text { Area } \\
{[p A * a]}\end{array}$ & $\begin{array}{l}\text { Height } \\
{[\mathrm{pA}]}\end{array}$ & $\begin{array}{c}\text { Area } \\
\frac{z}{z}\end{array}$ \\
\hline $\begin{array}{c}--1 \\
1\end{array}$ & 1.433 & vV & & $8.48968 \mathrm{e}-1$ & $5.84884 e-1$ & \\
\hline 2 & 1.691 & VB & 0.0144 & 28.16608 & 31.61176 & 12.98895 \\
\hline 3 & 13.279 & VB & 0.0440 & 1.10136 & $3.25549 \mathrm{e}-1$ & 0.50790 \\
\hline 4 & 14.070 & BB & 0.0310 & 1.75396 & $8.88927 e-1$ & 0.80885 \\
\hline 5 & 15.108 & $B P$ & 0.0324 & 1.22261 & $5.95961 \mathrm{e}-1$ & 0.56381 \\
\hline 6 & 15.414 & BB & 0.0304 & 146.27757 & 04477 & 67.45676 \\
\hline 7 & 16.011 & VB & 0.0312 & 37.47587 & 18.87050 & 17.28222 \\
\hline \multicolumn{4}{|c|}{ Totals : } & 16.84642 & 2235 & \\
\hline
\end{tabular}

Results obtained with enhanced integrator!

*** End of Report *** 


\begin{tabular}{|c|c|}
\hline $\begin{array}{l}\text { Injection Date } \\
\text { Sample Name } \\
\text { Acq. Operator }\end{array}$ & $\begin{array}{l}: \text { 10/6/2003 3:08:07 PM } \\
: \text { C-123-Crude } \\
: \text { jetena Glen }\end{array}$ \\
\hline Sequence File & 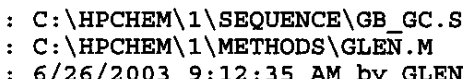 \\
\hline
\end{tabular}

Seq. Line : 1

Location : vial 1

Inj : 1

Inj Volume : $1 \mu$

Last changed : 6/26/2003 9:12:35 AM by GLEN
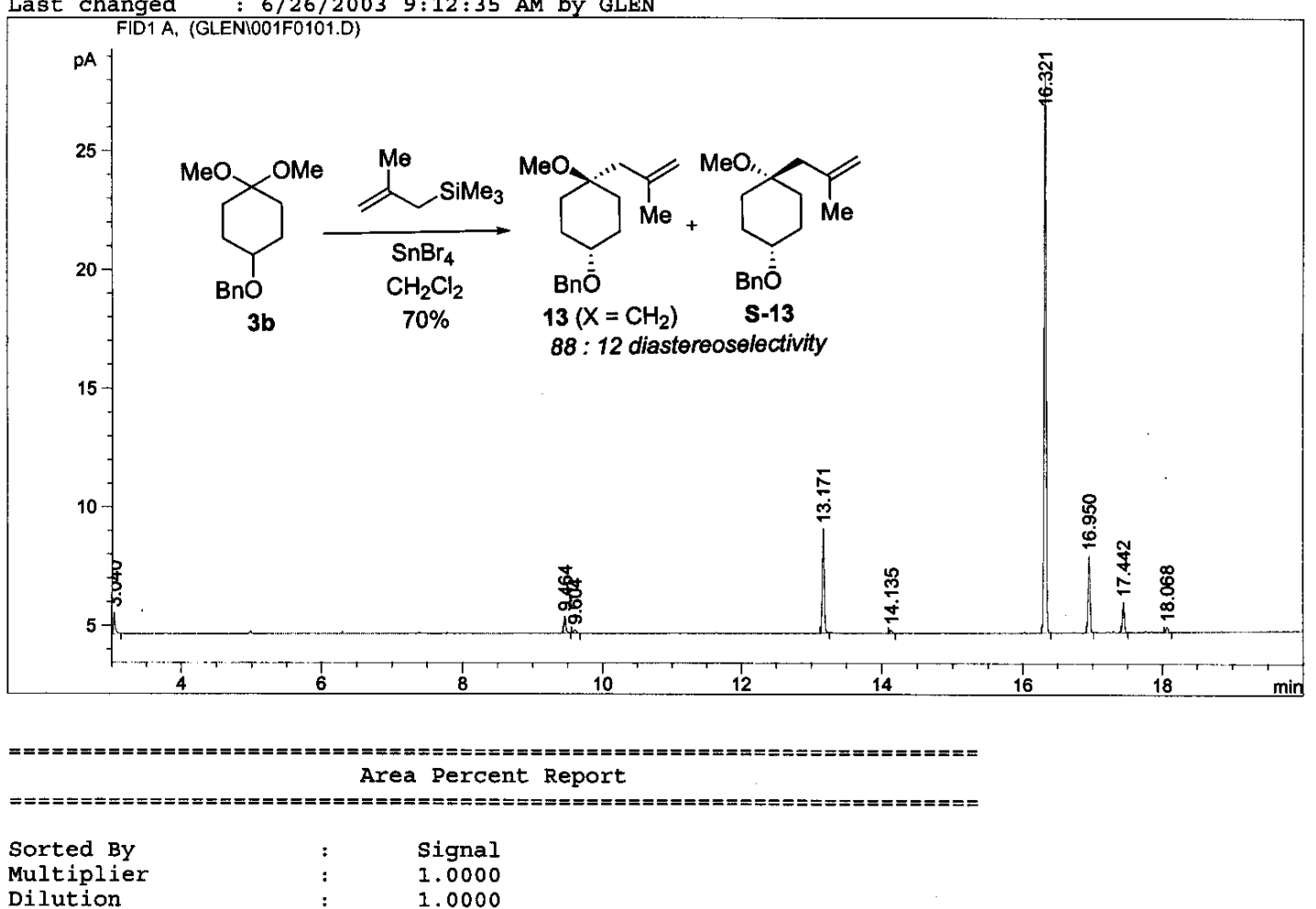

Signal 1: FID1 A,

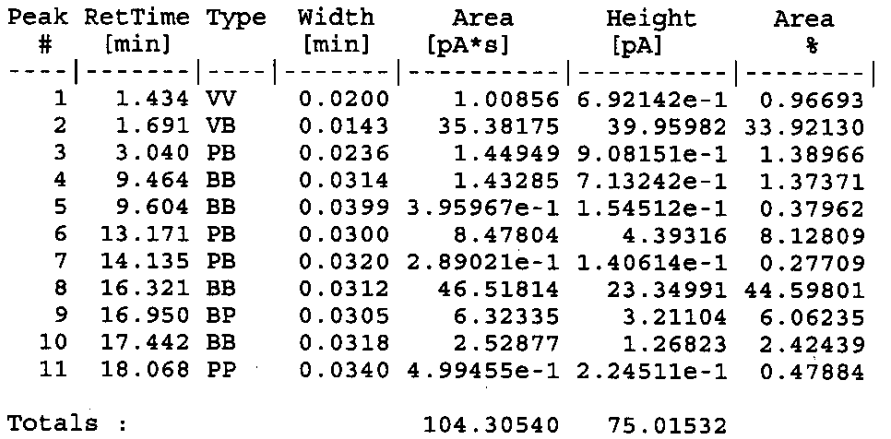

Results obtained with enhanced integrator! 
Data File C: $\backslash$ HPCHEM $\backslash 1 \backslash$ DATA $\backslash$ JELENA $\backslash 010 F 0101 . D$
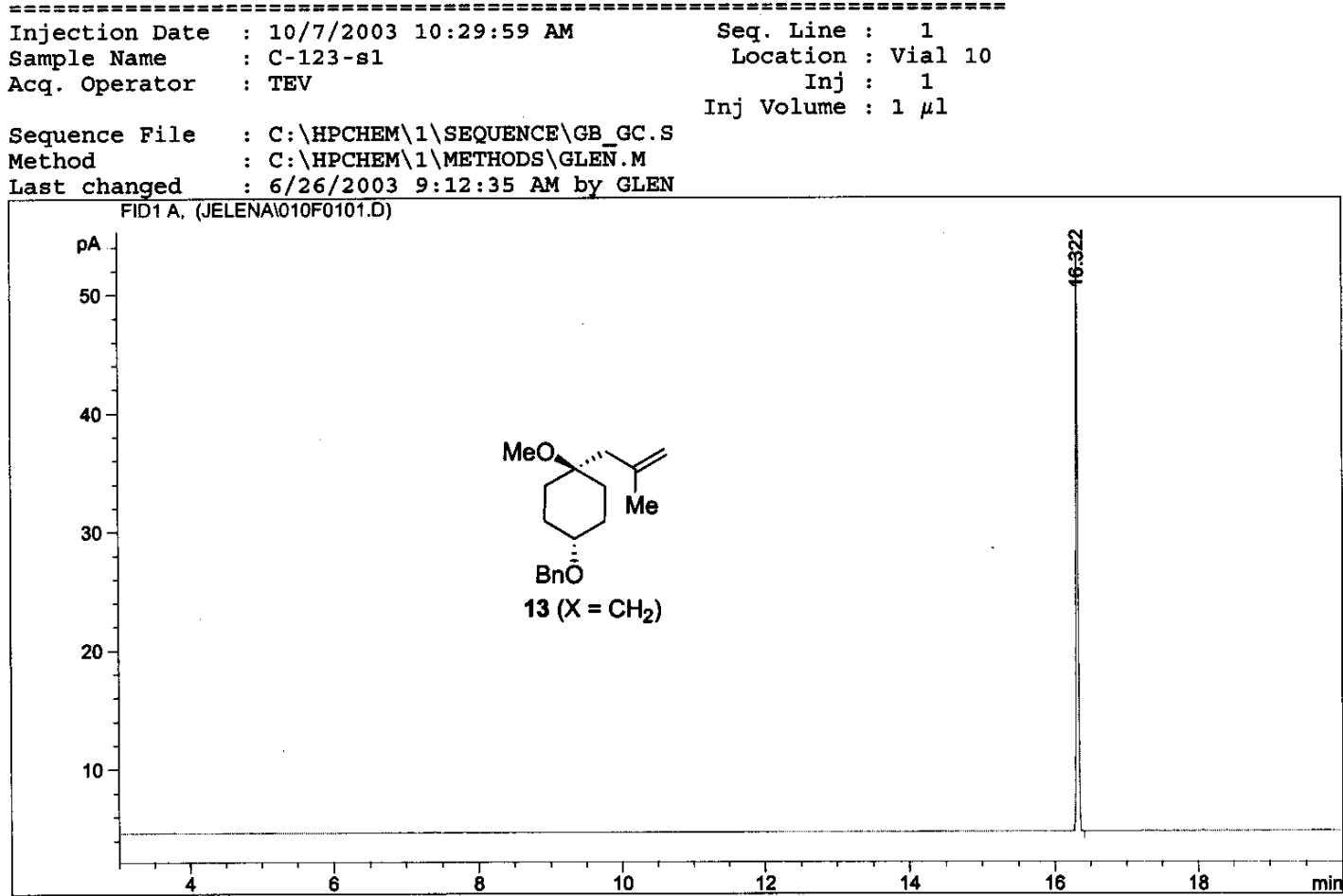

Area Percent Report

\begin{tabular}{|c|c|c|}
\hline Sorted BY & : & Signal \\
\hline Multiplier & : & 1.0000 \\
\hline Dilution & : & 1.0000 \\
\hline
\end{tabular}

Signal 1: FID1 A,

\begin{tabular}{|c|c|c|c|c|c|c|}
\hline $\begin{array}{c}\text { Peak } \\
\#\end{array}$ & $\begin{array}{l}\text { RetTime } \\
\text { [min] }\end{array}$ & Type & $\begin{array}{c}\text { Width } \\
\text { [min] }\end{array}$ & $\begin{array}{r}\text { Area } \\
{[p A * 8]}\end{array}$ & $\begin{array}{l}\text { Height } \\
{[\mathrm{pA}]}\end{array}$ & $\begin{array}{c}\text { Area } \\
\frac{8}{8}\end{array}$ \\
\hline & & & & & & \\
\hline 1 & 1.357 & BV & 0.0183 & $2.67329 e-1$ & $2.03497 e-1$ & 0.19246 \\
\hline 2 & 1.433 & VB & 0.0182 & 1.14411 & $8.80396 e-1$ & 0.82370 \\
\hline 3 & 1.690 & VB & 0.0144 & 43.86238 & 49.21237 & 31.57878 \\
\hline 4 & 16.322 & BB & 0.0314 & 93.62447 & 47.68884 & 67.40506 \\
\hline \multicolumn{4}{|c|}{ Totals : } & 138.89829 & 97.98510 & \\
\hline
\end{tabular}

Results obtained with enhanced integrator! 

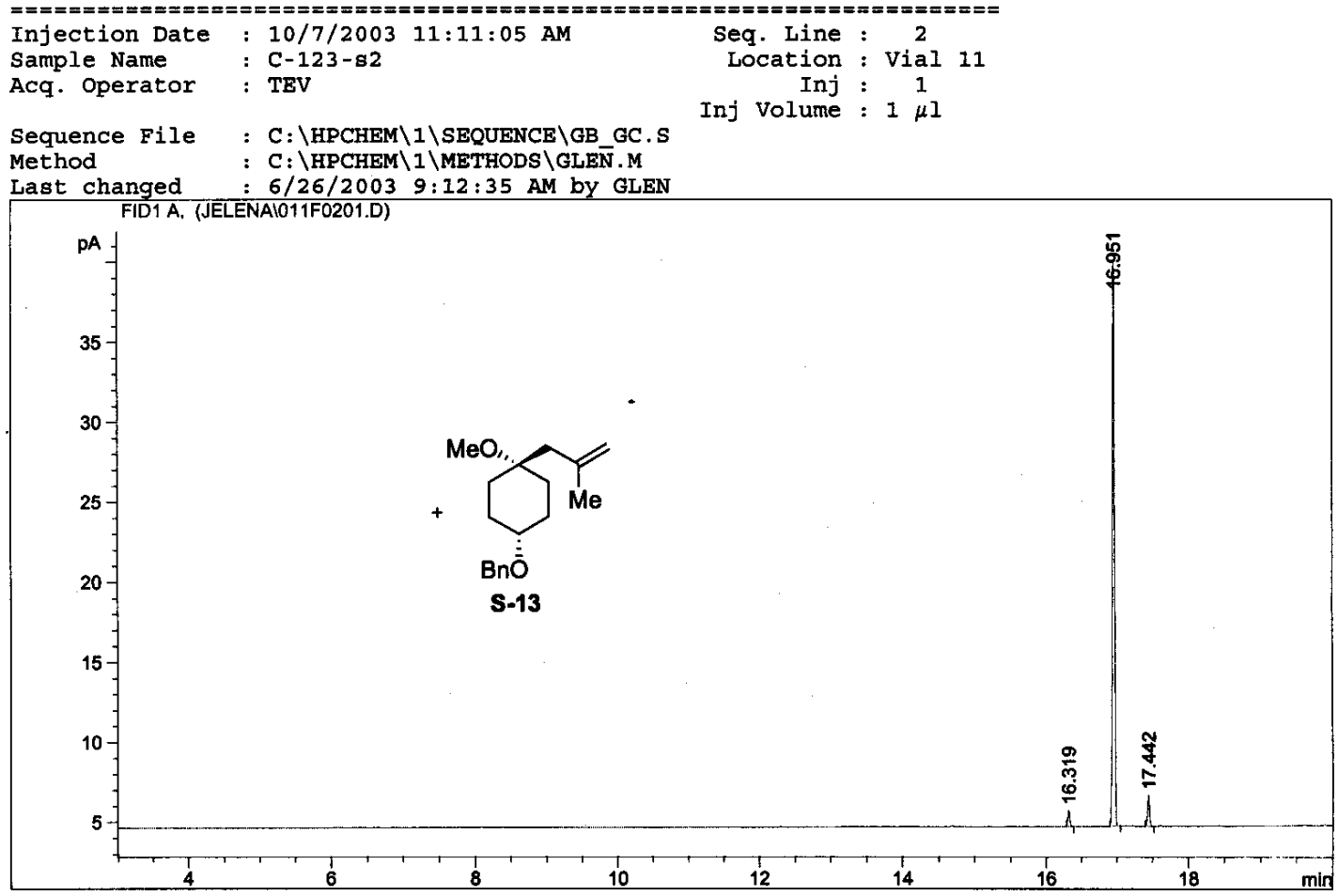

Area Percent Report

\begin{tabular}{lcr}
\multicolumn{1}{c}{} & \multicolumn{2}{c}{ Area Percent } \\
Sorted By & $:$ & Signal \\
Multiplier & $:$ & 1.0000 \\
Dilution & $:$ & 1.0000
\end{tabular}

Signal 1: FID1 A,

\begin{tabular}{|c|c|c|c|c|c|c|}
\hline $\begin{array}{c}\text { Peak } \\
\#\end{array}$ & $\begin{array}{l}\text { RetTime } \\
\text { [min] }\end{array}$ & Type & $\begin{array}{c}\text { width } \\
\text { [min] }\end{array}$ & $\begin{array}{r}\text { Area } \\
{[p A * B]}\end{array}$ & $\begin{array}{l}\text { Height } \\
\text { [pA] }\end{array}$ & $\begin{array}{c}\text { Area } \\
:\end{array}$ \\
\hline 1 & 1.357 & BV & 0.0158 & $2.47211 e-1$ & $2.25661 e-1$ & 0.19898 \\
\hline 2 & 1.433 & VB & 0.0177 & 1.21069 & $9.61938 \mathrm{e}-1$ & 0.97451 \\
\hline 3 & 1.690 & VB & 0.0139 & 47.42224 & 52.96078 & 38.17103 \\
\hline 4 & 16.319 & BB & 0.0310 & 2.04513 & 1.03859 & 1.64616 \\
\hline 5 & 16.951 & BB & 0.0309 & 69.47214 & 35.36600 & 55.91939 \\
\hline 6 & 17.442 & BP & 0.0309 & 3.83881 & 1.95354 & 3.08993 \\
\hline \multicolumn{4}{|c|}{ Totals : } & 124.23622 & 92.50651 & \\
\hline
\end{tabular}

Results obtained with enhanced integrator!

$\star \star \star$ End of Report $\star \star \star$ 


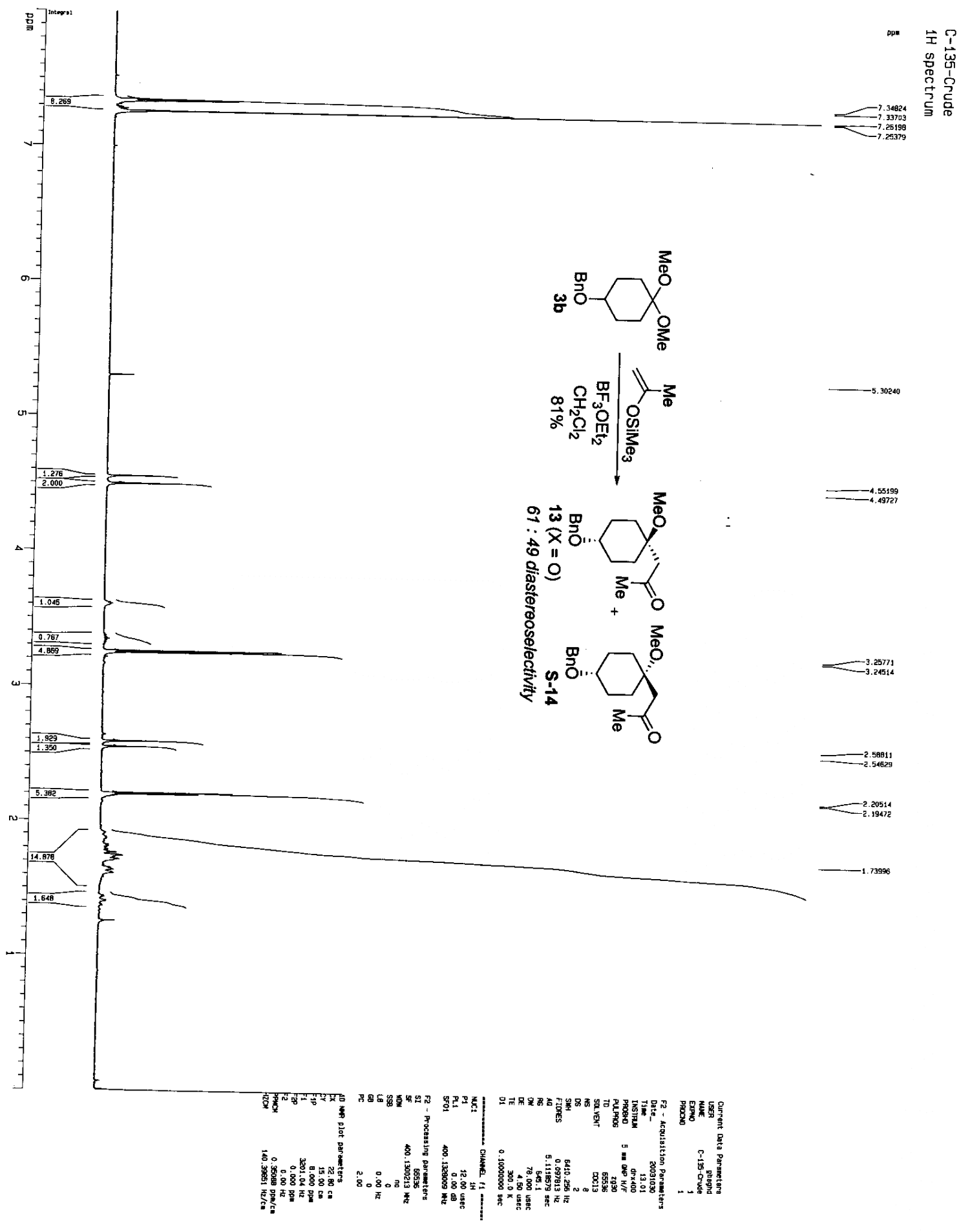




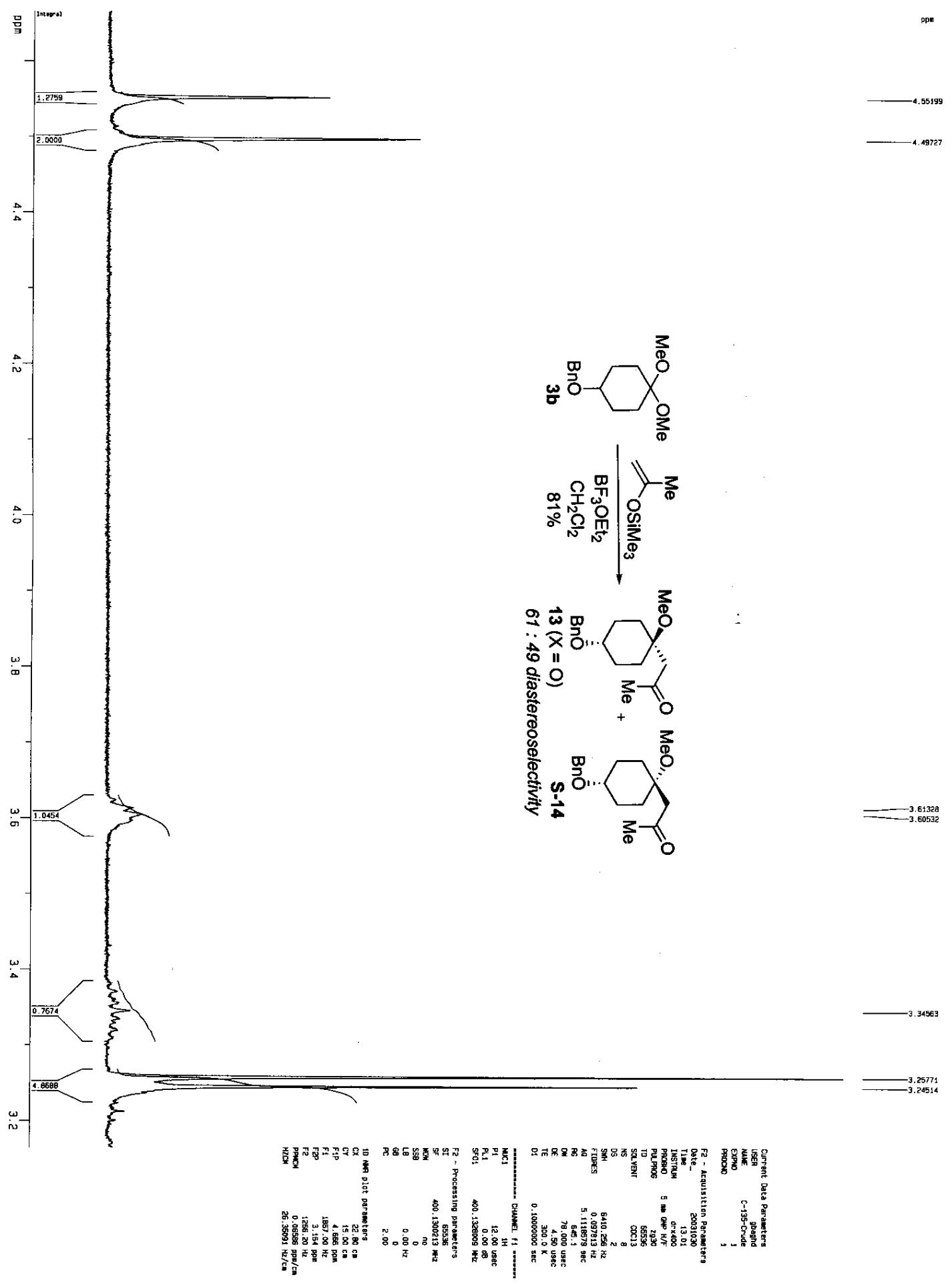




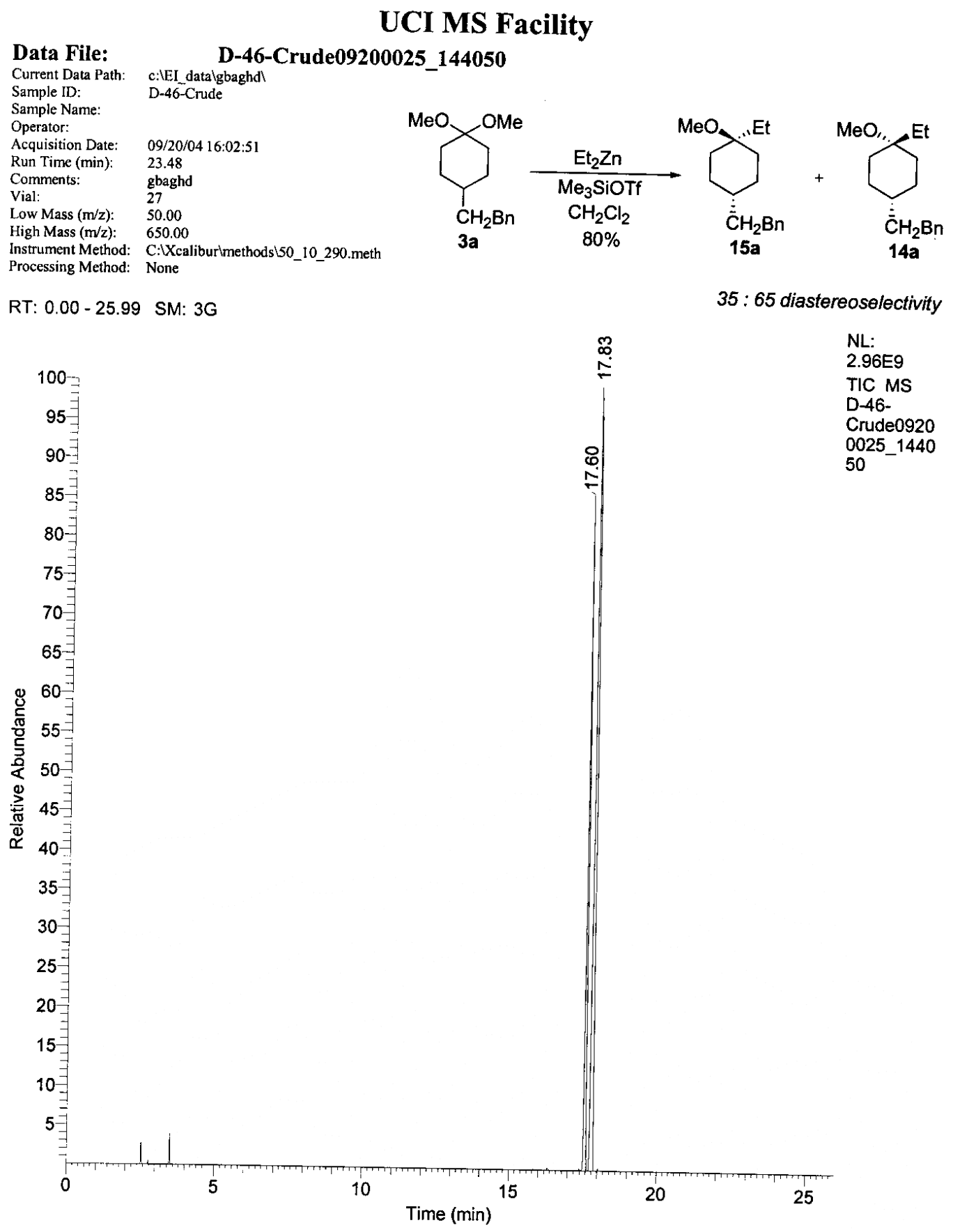

Page 1 of 3 


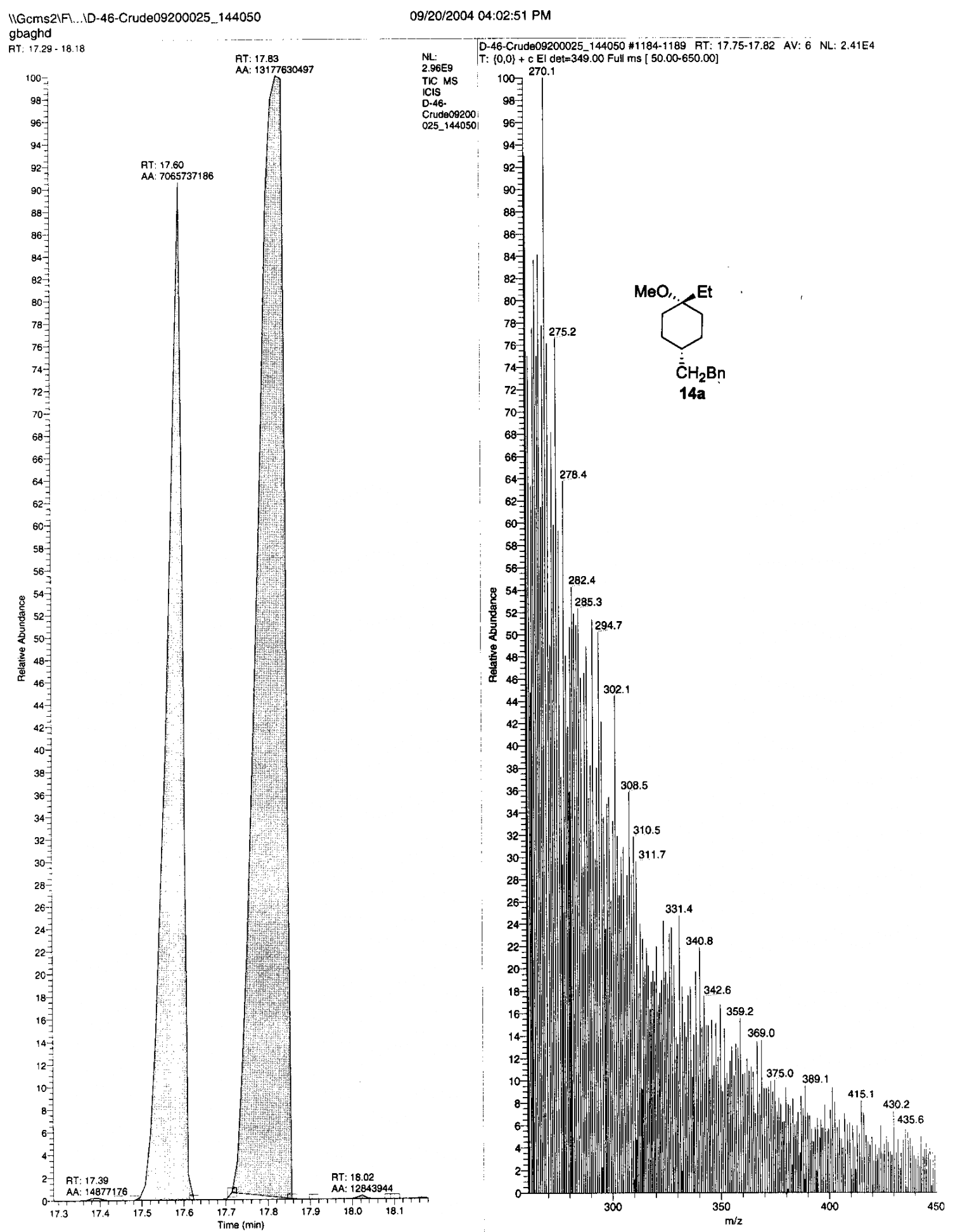




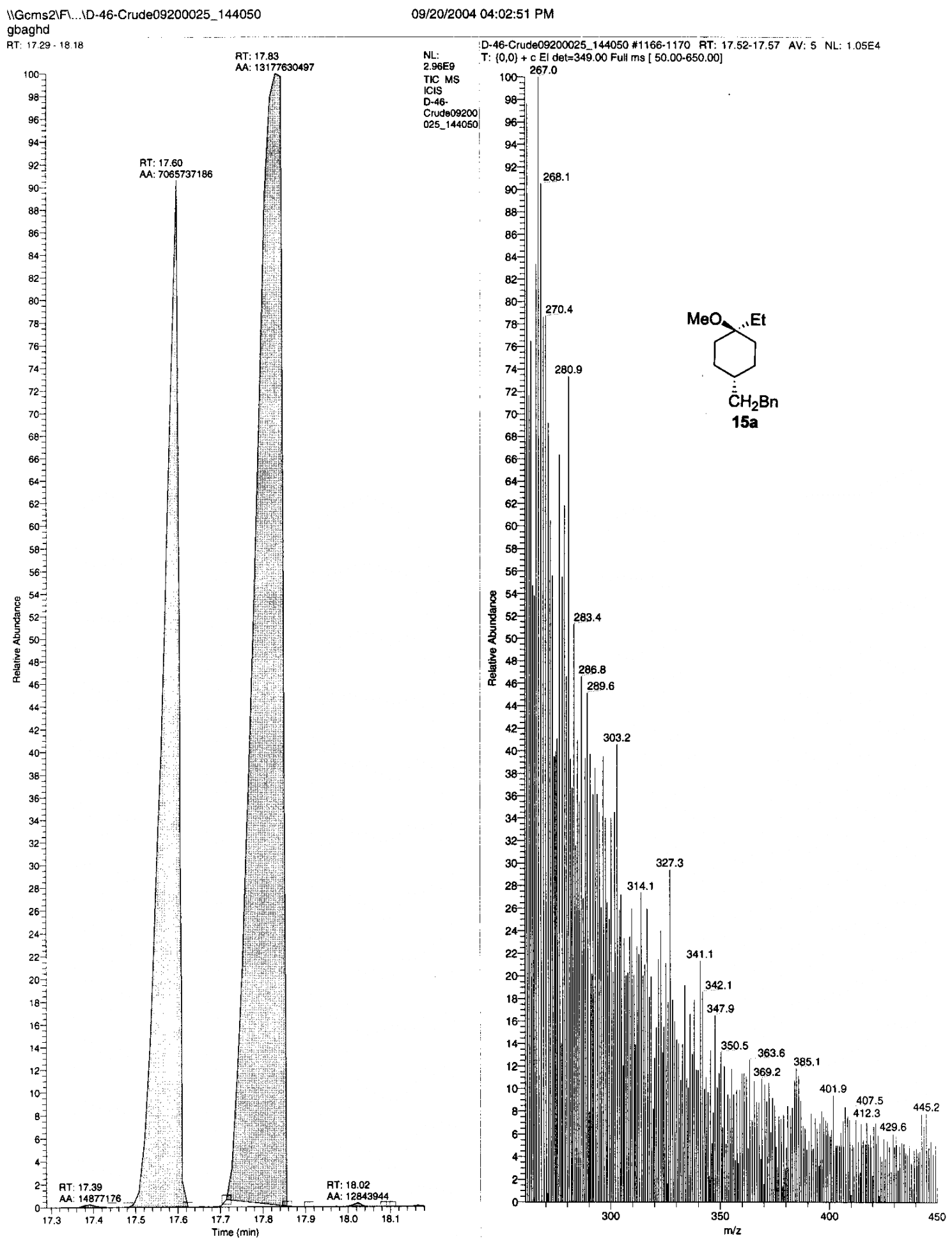



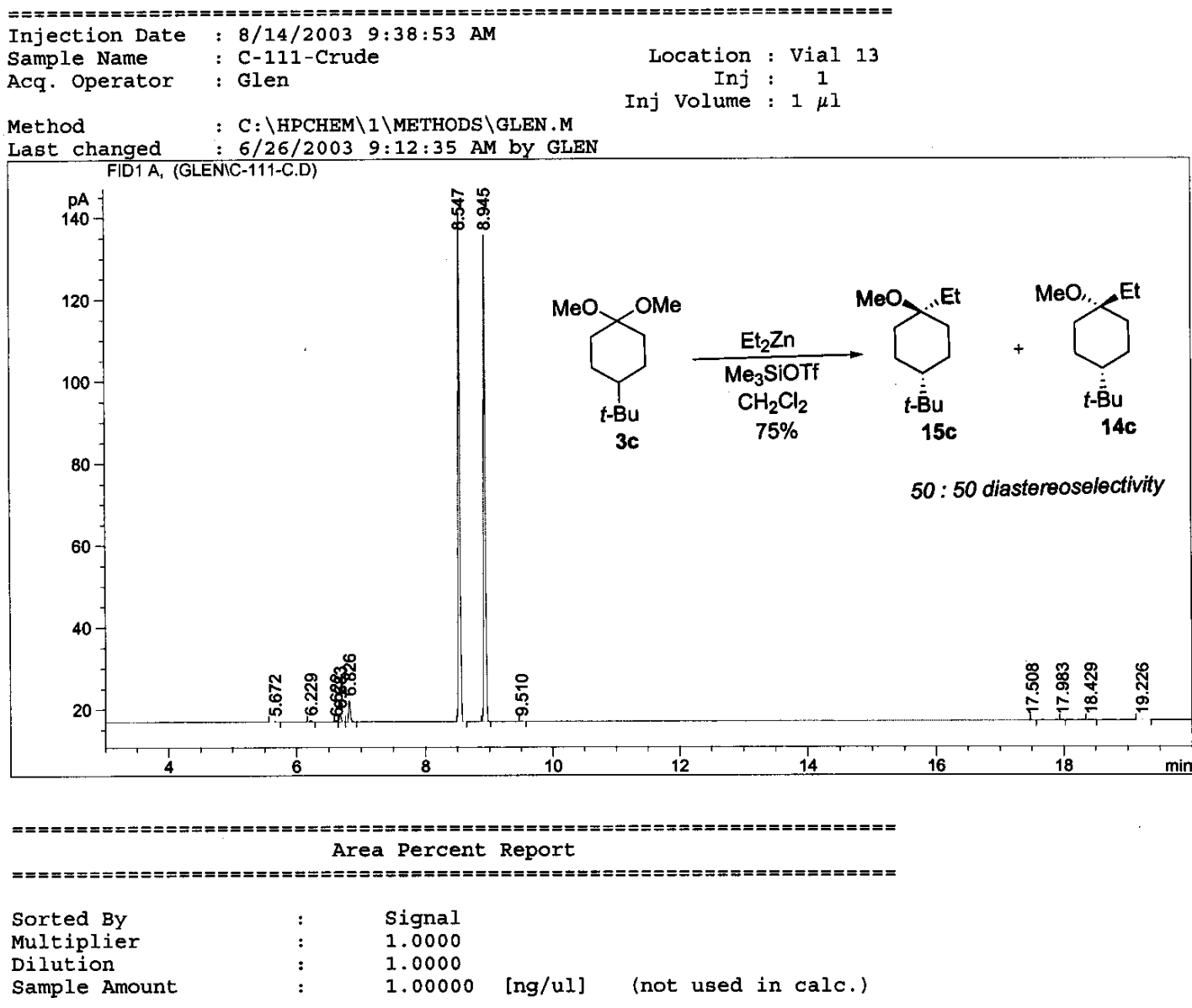

Signal 1: FID1 A,

\begin{tabular}{|c|c|c|c|c|c|c|}
\hline eak & $\begin{array}{l}\text { RetTime } \\
\text { [min] }\end{array}$ & Type & $\begin{array}{l}\text { Width } \\
\text { [min] }\end{array}$ & $\begin{array}{r}\text { Area } \\
{\left[\mathrm{pA}^{*} \mathrm{~s}\right]}\end{array}$ & $\begin{array}{l}\text { Height } \\
\text { [pA] }\end{array}$ & $\underset{z}{\text { Area }}$ \\
\hline 1 & 1.399 & VP & 0.0121 & $2.9111^{\prime}$ & 3. $95379 \mathrm{e}-1$ & 0.05702 \\
\hline 2 & 1.442 & vV & 0.0224 & $4 e-1$ & & \\
\hline 3 & 1.692 & VB & 0.0144 & 2555 & 1133 & 5.52816 \\
\hline 4 & 5.6 & $\mathrm{BP}$ & 0.0 & 3.977 & 1.87 & \\
\hline 5 & 6.2 & VB & 0.0 & 8.521 & 4.3 & 690 \\
\hline 6 & 6.622 & BV & 0.0 & 4.611 & 2.45 & 9032 \\
\hline 7 & 6.6 & VP & & 711 & & 0049 \\
\hline 8 & & VB & & & & \\
\hline & 8 & BB & 0.0304 & 237 & 123 & 46.55651 \\
\hline 10 & 8.9 & BB & & 226 & & 44.27 \\
\hline 11 & 9.5 & PP & 0.0321 & 2.5710 & 1.1716 & 0.05036 \\
\hline 12 & 17.5 & BP & & 2.31 & 9.96 & 542 \\
\hline 13 & & BV & & 2.811 & 1.138956 & 0.05507 \\
\hline 14 & 18.429 & VB & 0.0448 & $6.30817 \mathrm{e}-1$ & $1.92293 \mathrm{e}-1$ & 0.12355 \\
\hline
\end{tabular}




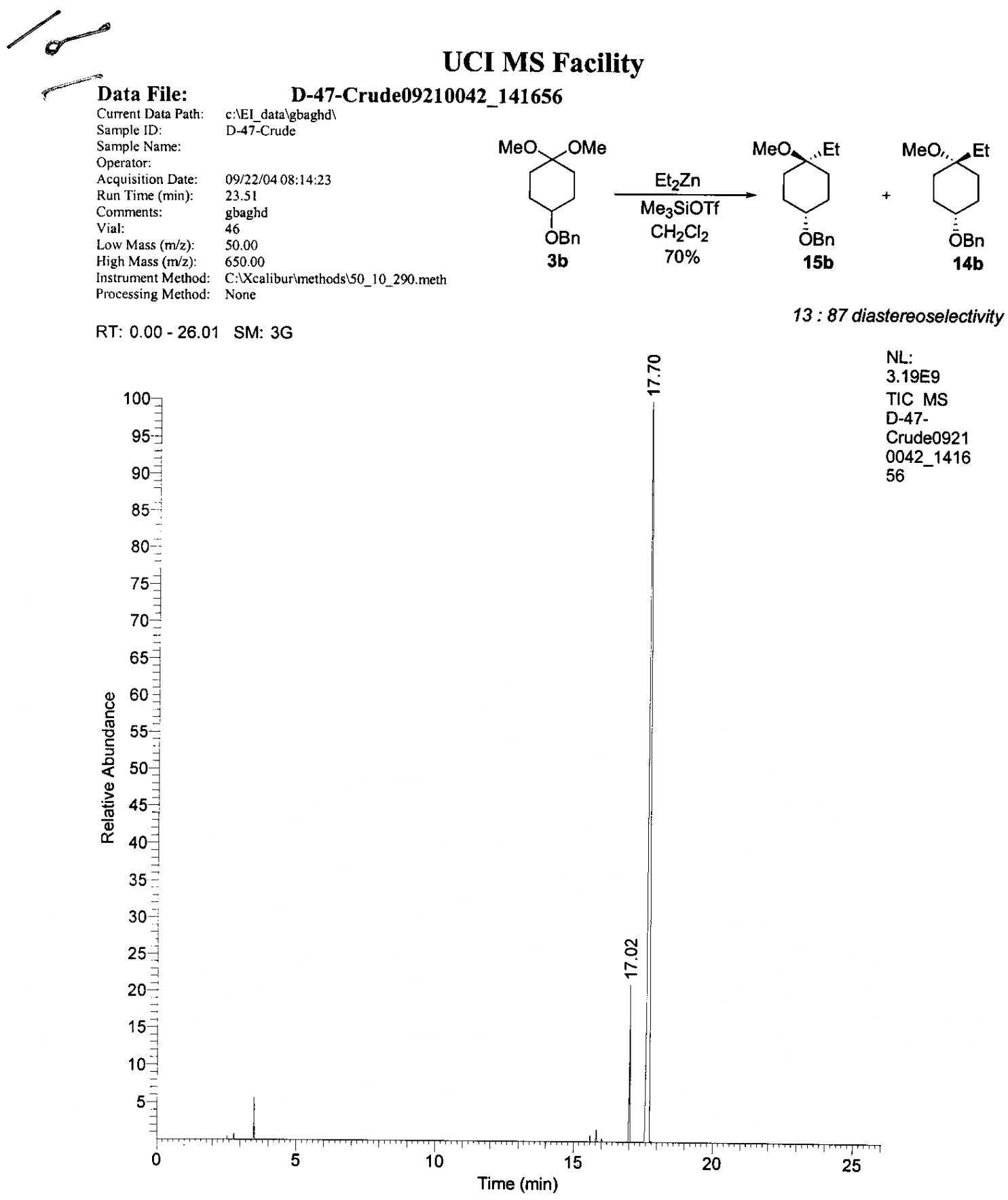

Page 1 of 3 


\section{UCI MS Facility}

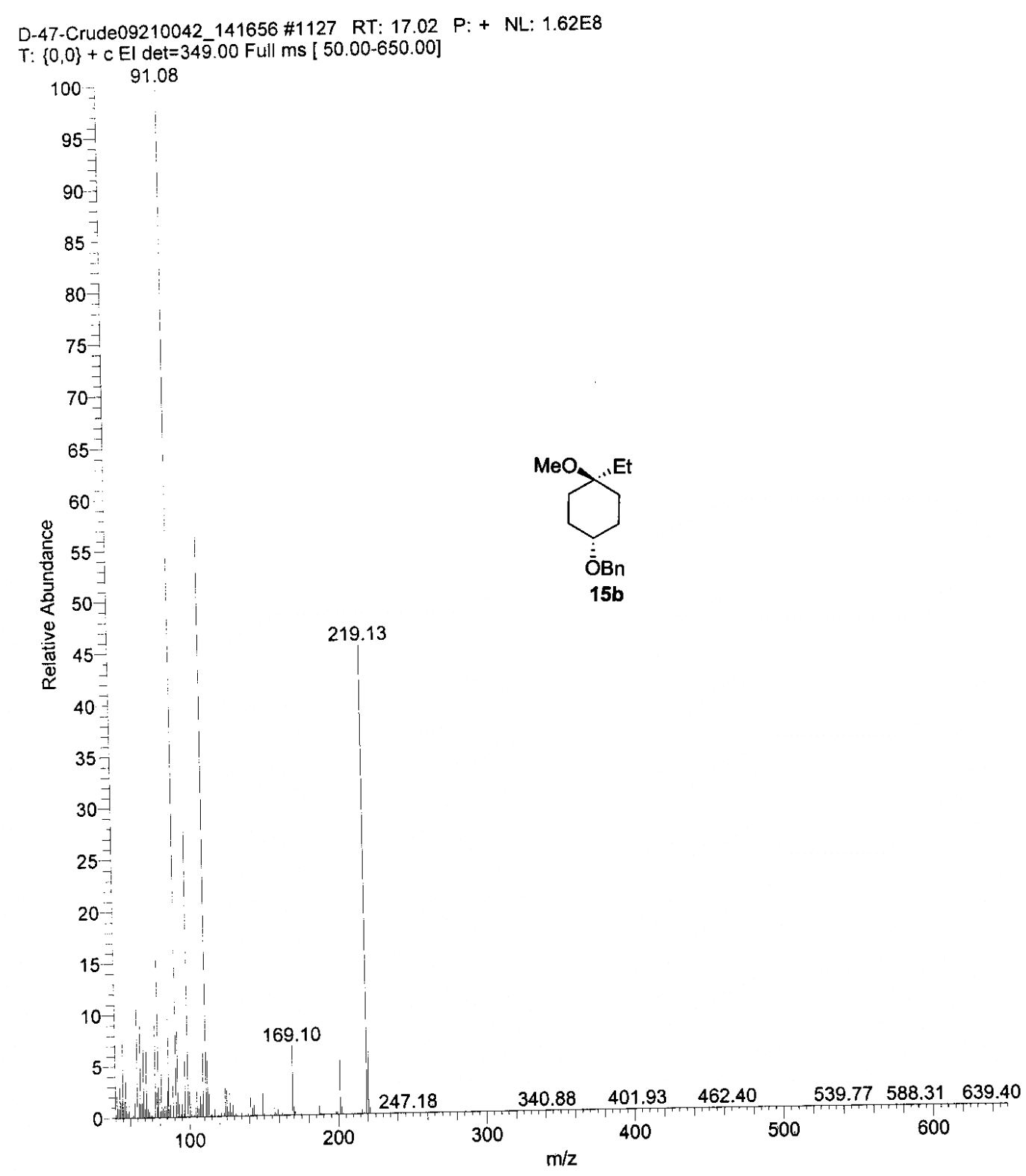

Page 2 of 3 


\section{UCI MS Facility}

J-47-Crude09210042 141656 \#1180 RT: 17.70 P: + NL: 2.58E8

T: $\{0,0\}+c$ El det $=34 \overline{9} .00$ Full ms $[50.00-650.00]$

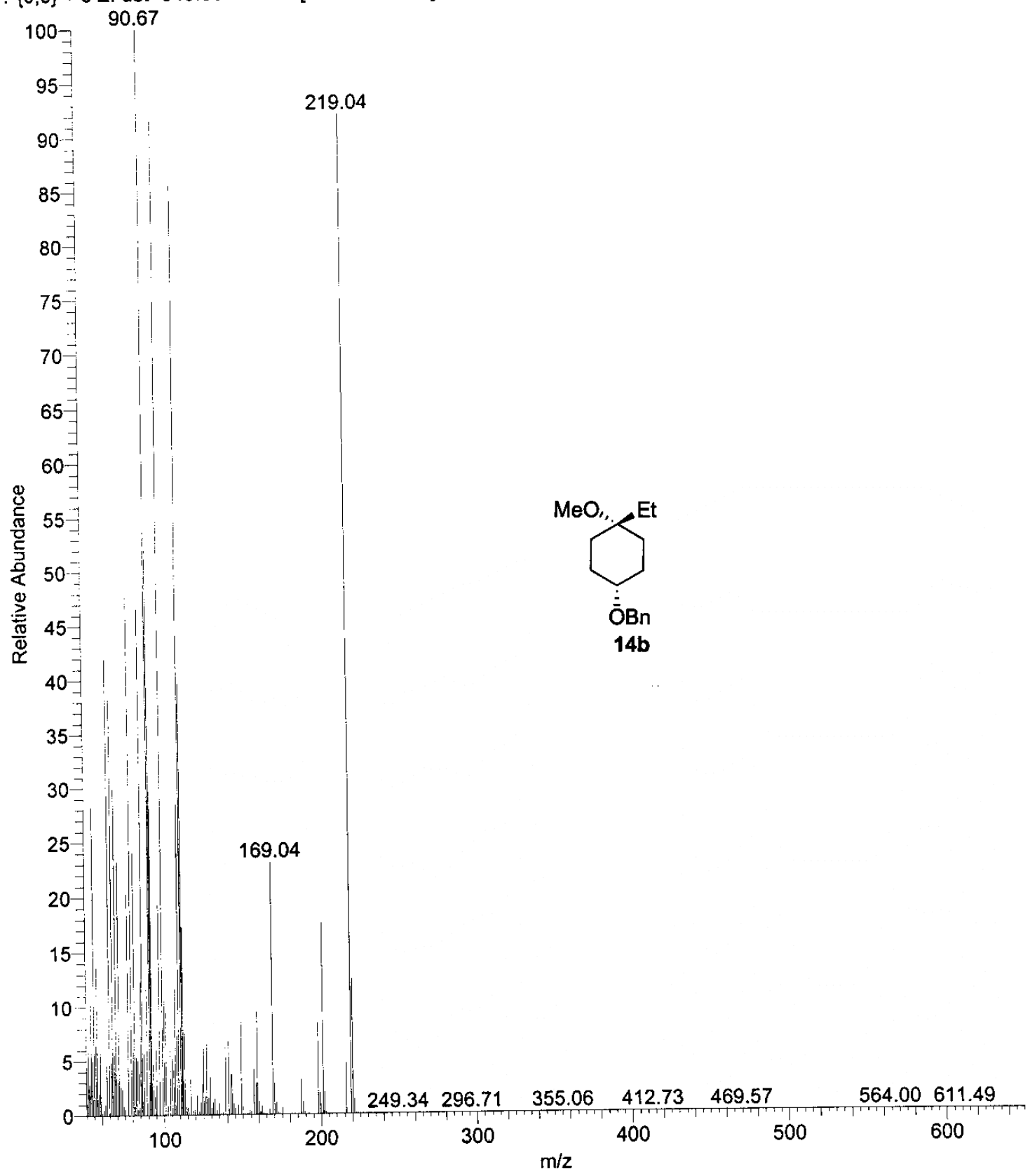




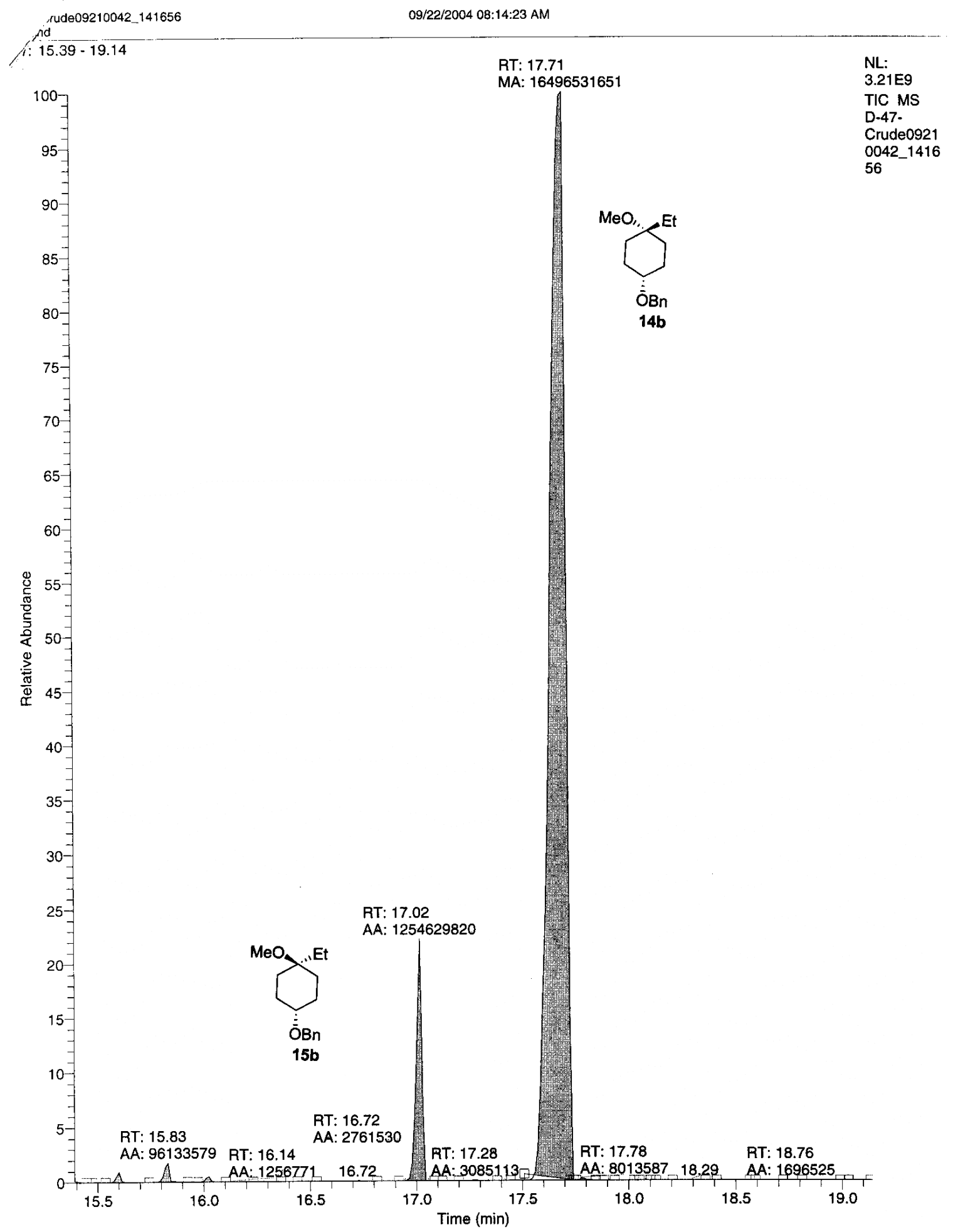




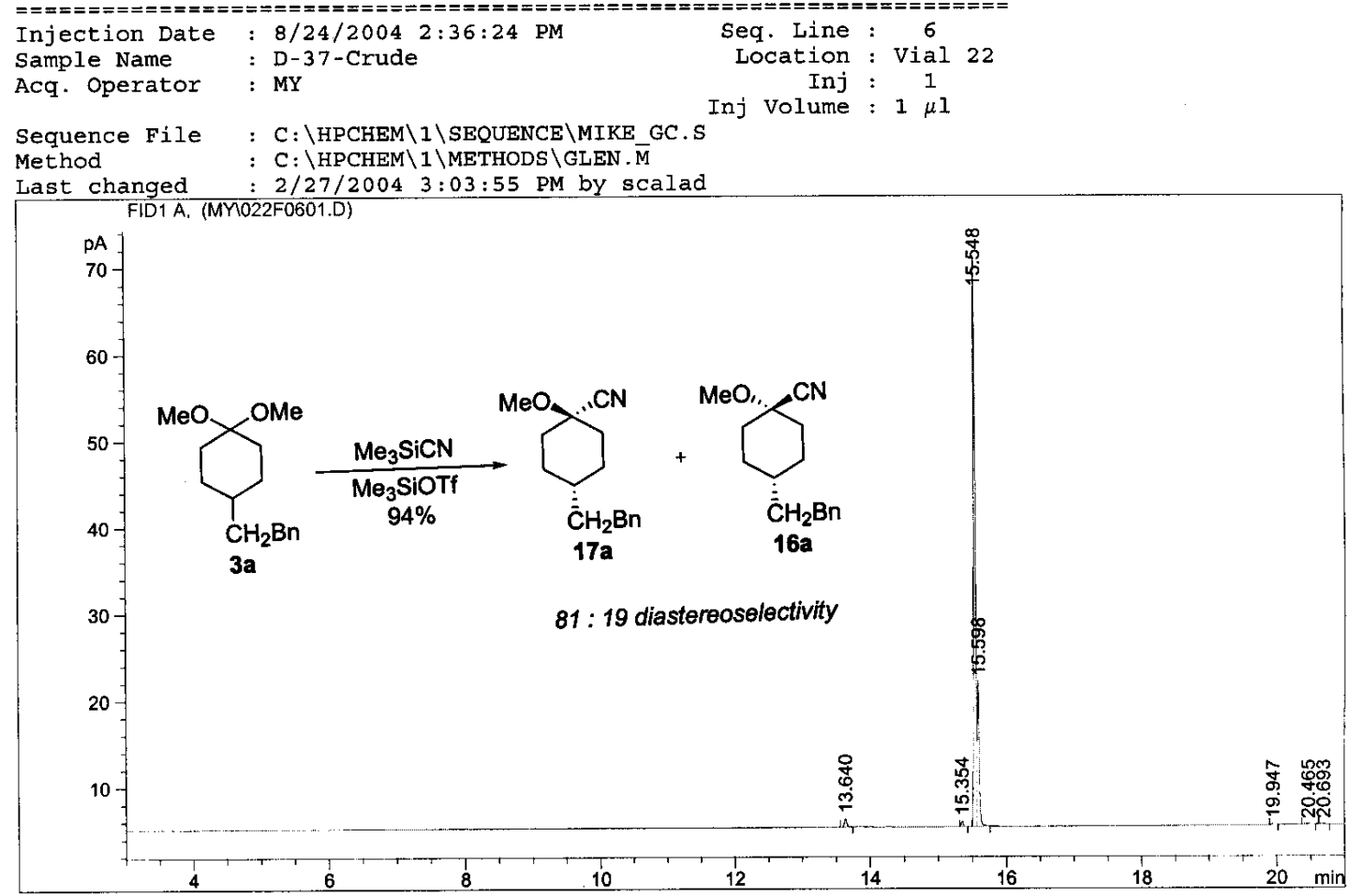

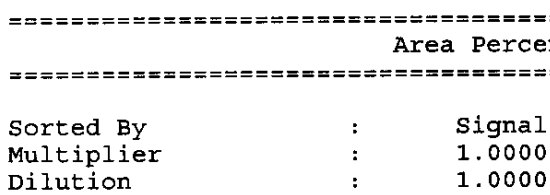

Signal 1: FID1 A,

\begin{tabular}{|c|c|c|c|c|c|c|}
\hline $\begin{array}{c}\text { Peak } \\
\#\end{array}$ & $\begin{array}{c}\text { RetTime } \\
\text { [min] }\end{array}$ & Type & $\begin{array}{l}\text { Width } \\
\text { [min] }\end{array}$ & $\begin{array}{r}\text { Area } \\
{\left[\mathrm{pA}^{*} \mathrm{~s}\right]}\end{array}$ & $\begin{array}{l}\text { Height } \\
\text { [pA] }\end{array}$ & $\begin{array}{c}\text { Area } \\
\frac{8}{8}\end{array}$ \\
\hline & & & & & & $-\cdots---$ \\
\hline 1 & 1.342 & $B P$ & 0.0119 & $3.14474 \mathrm{e}-1$ & $4.11534 \mathrm{e}-1$ & 0.14347 \\
\hline 2 & 1.418 & PB & 0.0127 & 1.55639 & 1.97942 & 0.71005 \\
\hline 3 & 1.677 & PB & 0.0140 & 48.68509 & 54.32571 & 22.21092 \\
\hline 4 & 13.640 & PB & 0.0385 & 2.41318 & $9.88010 \mathrm{e}-1$ & 1.10093 \\
\hline 5 & 15.354 & $\mathrm{BB}$ & 0.0310 & 1.24726 & $6.32535 e-1$ & 0.56902 \\
\hline 6 & 15.548 & BV & 0.0310 & 132.64091 & 65.75965 & 60.51292 \\
\hline 7 & 15.598 & VB & 0.0285 & 31.54790 & 16.69423 & 14.39266 \\
\hline 8 & 19.947 & BB & 0.0406 & $3.17301 e-1$ & $1.20934 e-1$ & 0.14476 \\
\hline 9 & 20.465 & $\mathrm{BP}$ & 0.0630 & $2.57205 \mathrm{e}-1$ & $5.08376 e-2$ & 0.11734 \\
\hline 10 & 20.693 & BP & 0.0507 & $2.14657 e-1$ & $5.30175 e-2$ & 0.09793 \\
\hline & & & & 219.19438 & & \\
\hline
\end{tabular}

Results obtained with enhanced integrator! 


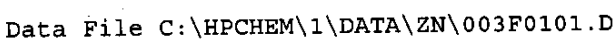

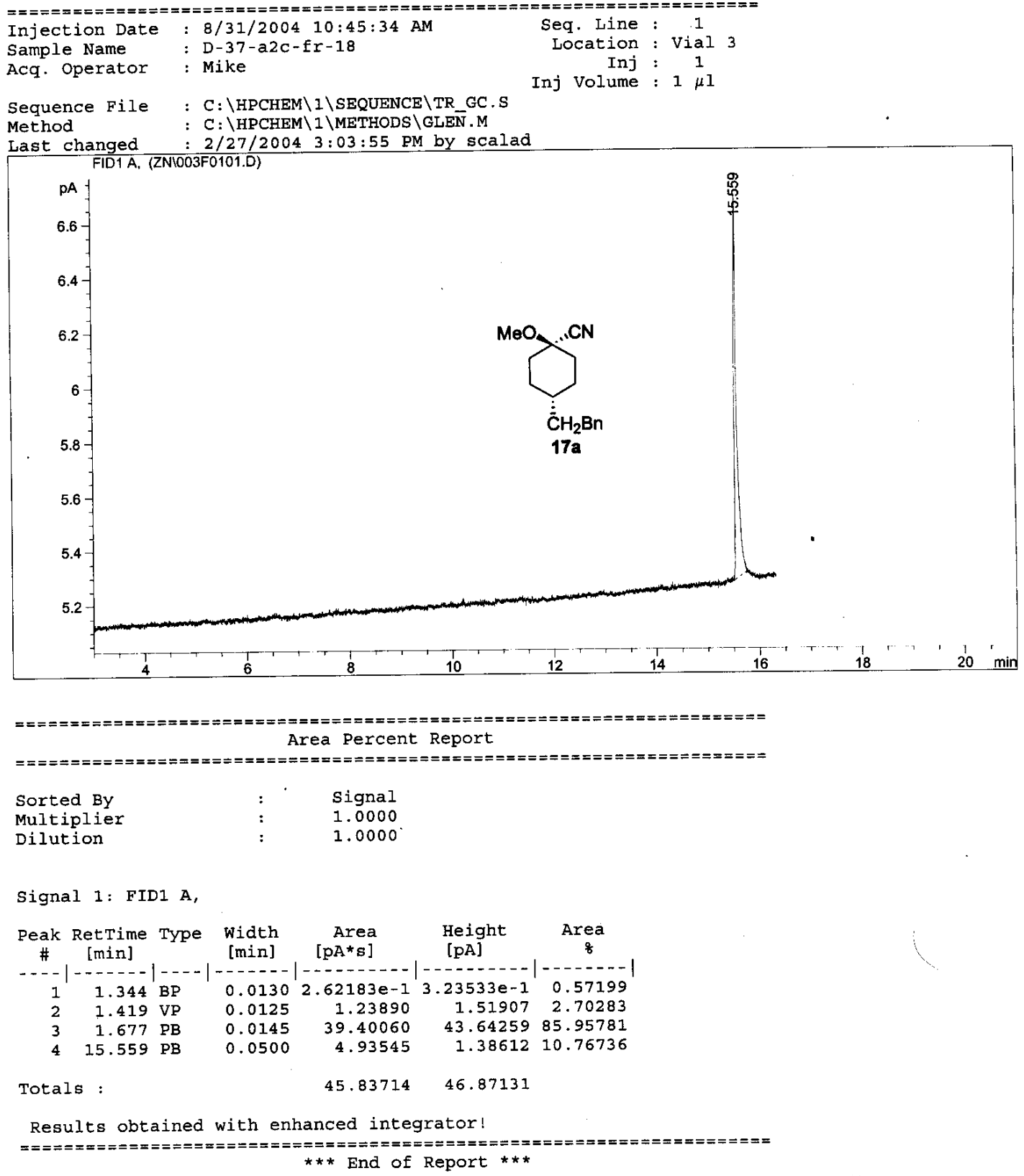


Data File C: $\backslash$ HPCHEM $\backslash 1 \backslash D A T A \backslash S A C \backslash 013 F 0301 . D$

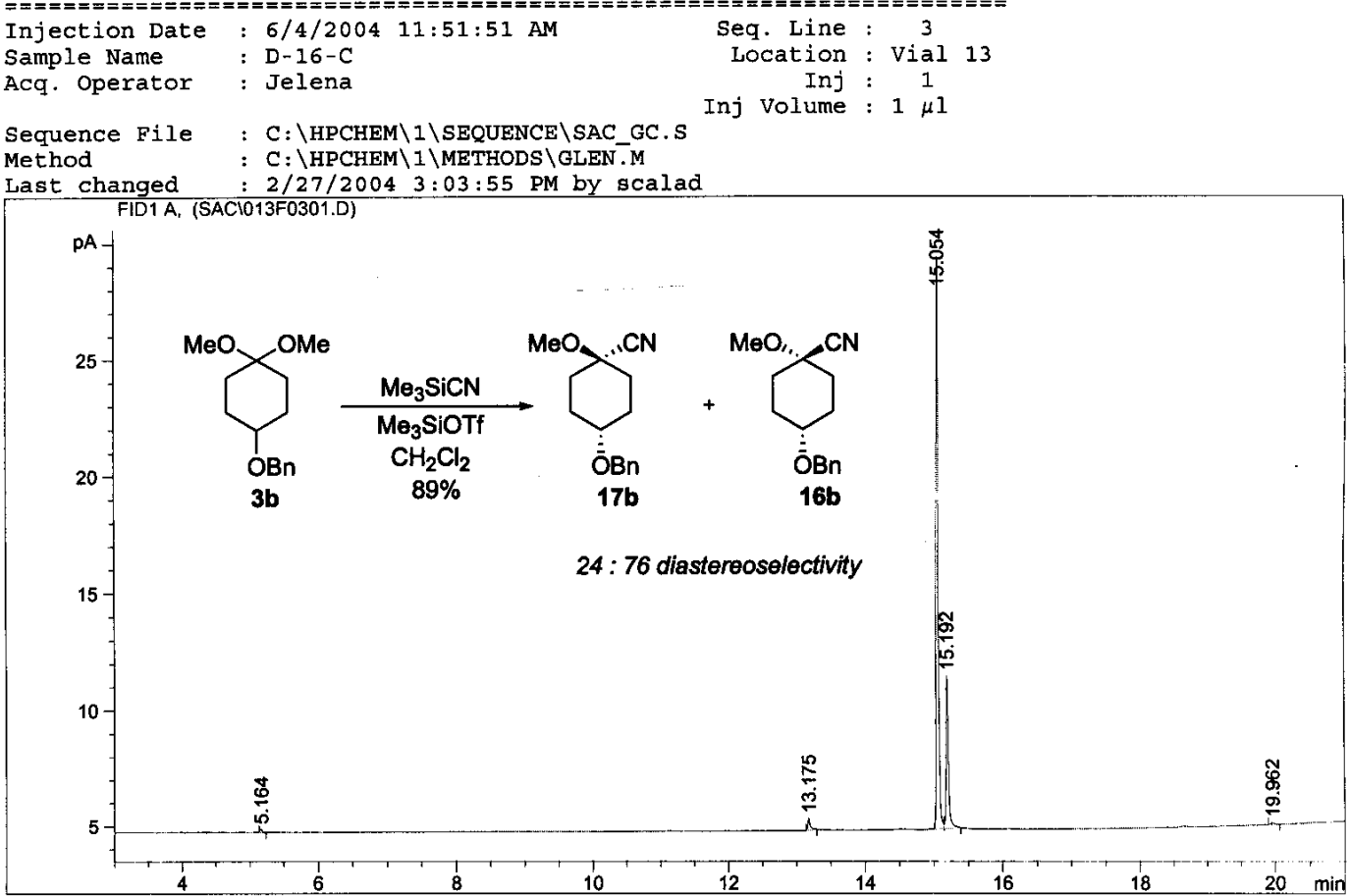

\begin{tabular}{|c|c|c|}
\hline Sorted By & : & Signal \\
\hline Multiplier & : & 1.0000 \\
\hline Dilution & : & 1.0000 \\
\hline
\end{tabular}

Signal 1: FIDI A

\begin{tabular}{|c|c|c|c|c|c|c|}
\hline $\begin{array}{c}\text { Peak } \\
\#\end{array}$ & $\begin{array}{c}\text { RetTime } \\
\text { [min] }\end{array}$ & Type & $\begin{array}{l}\text { Width } \\
\text { [min] }\end{array}$ & $\begin{array}{r}\text { Area } \\
{\left[p A^{*} \mathbf{S}\right]}\end{array}$ & $\begin{array}{l}\text { Height } \\
{[\mathrm{pA}]}\end{array}$ & $\begin{array}{c}\text { Area } \\
\frac{q}{\gamma}\end{array}$ \\
\hline 1. & 1.344 & PP & 0.0122 & $3.17397 e-1$ & $4.02356 \mathrm{e}-1$ & 0.36644 \\
\hline 2 & 1.420 & VB & 0.0130 & 1.24392 & 1.53052 & 1.43612 \\
\hline 3 & 1.680 & PB & 0.0149 & 16.23962 & 17.39316 & 18.74887 \\
\hline 4 & 5.164 & BB & 0.0316 & $2.79409 \mathrm{e}-1$ & $1.38259 \mathrm{e}-1$ & 0.32258 \\
\hline 5 & 13.175 & PB & 0.0413 & 1.44322 & $5.20637 e-1$ & 1.66622 \\
\hline 6 & 15.054 & PV & 0.0322 & 50.76133 & 24.49549 & 58.60467 \\
\hline 7 & 15.192 & VB & 0.0368 & 15.93543 & 6.57037 & 18.39768 \\
\hline 8 & 19.962 & BP & 0.0624 & $3.96200 \mathrm{e}-1$ & 8. $65691 \mathrm{e}-2$ & 0.45742 \\
\hline
\end{tabular}

Totals: $\quad 86.61653 \quad 51.13737$

Results obtained with enhanced integrator! 


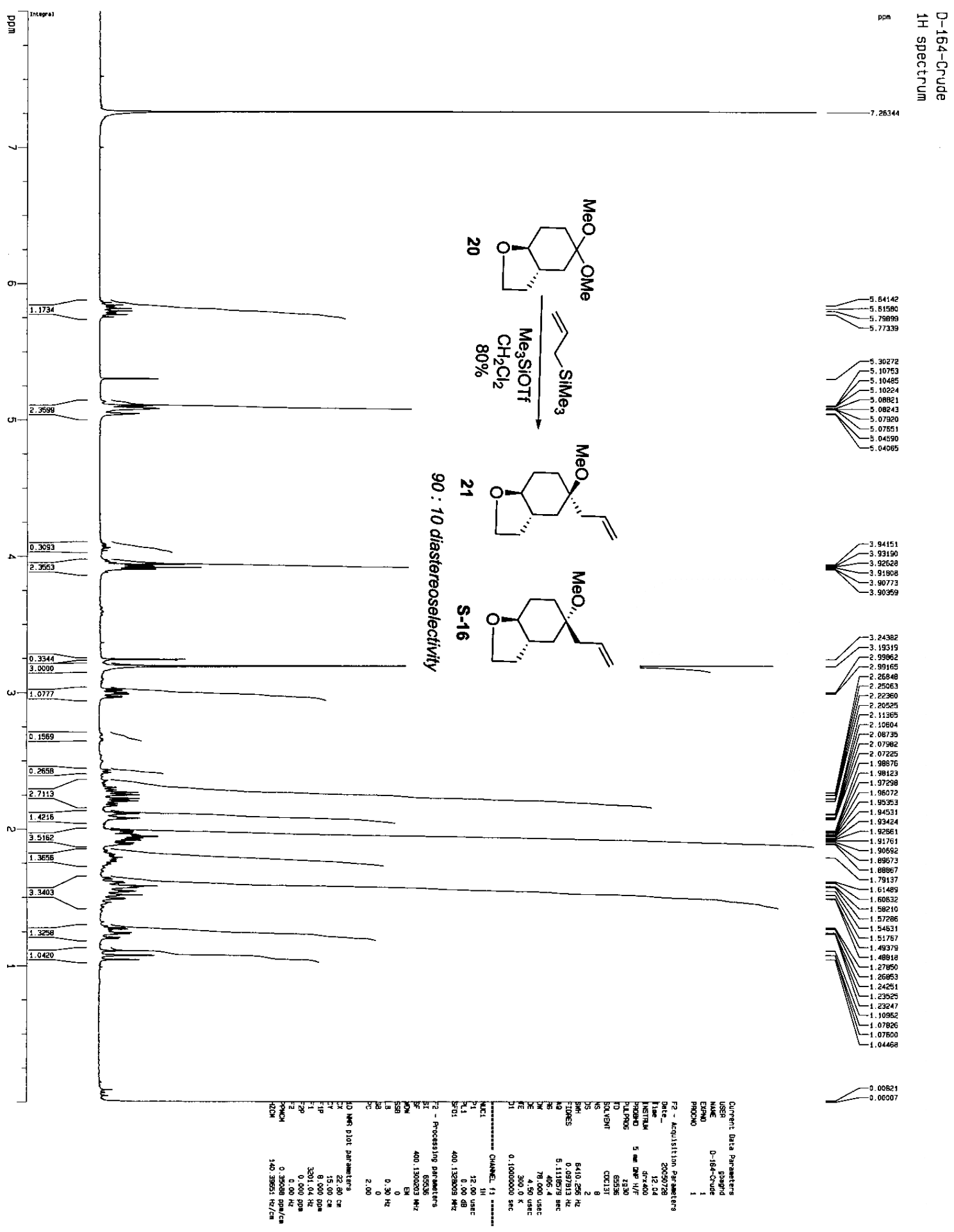




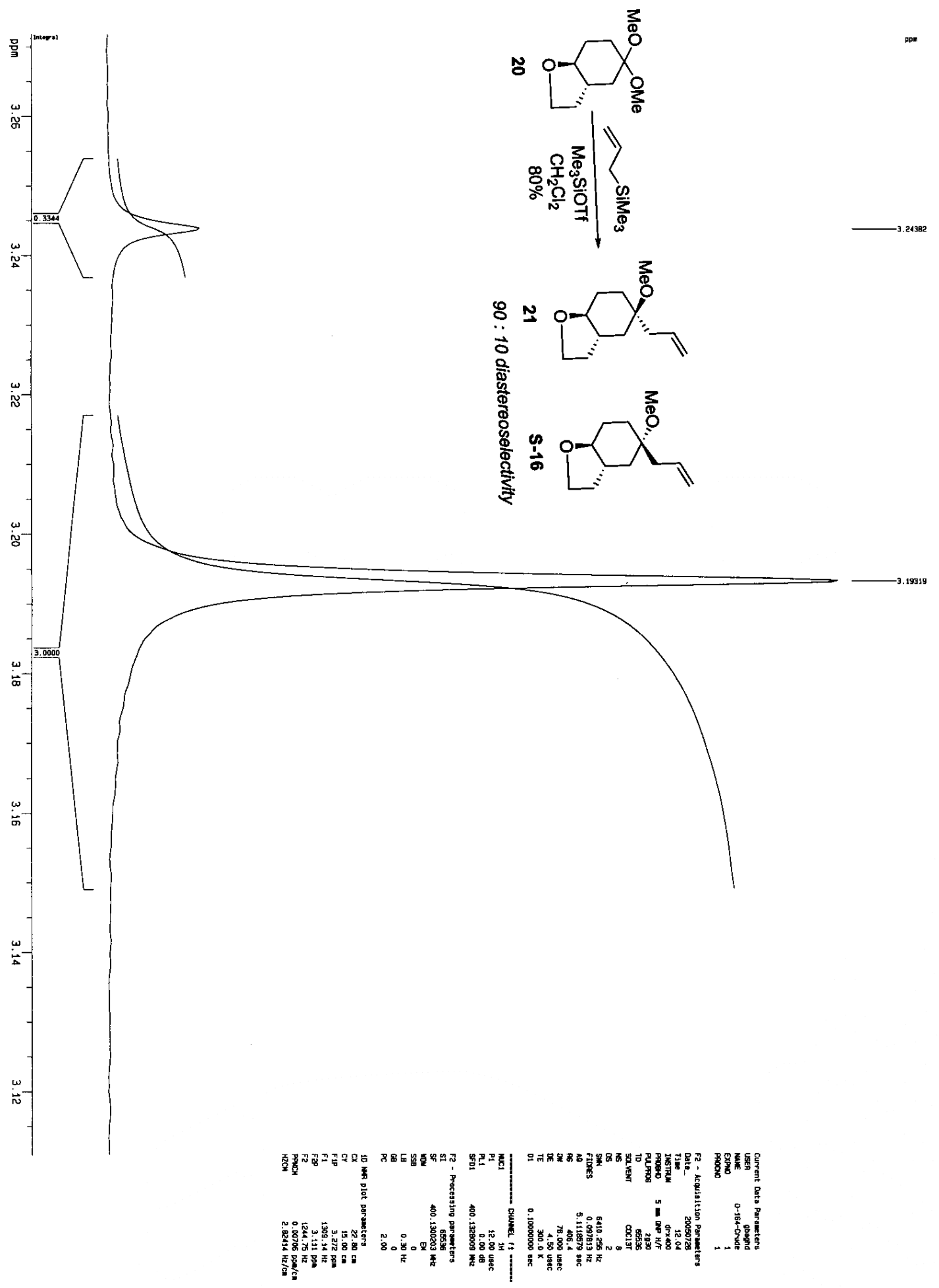



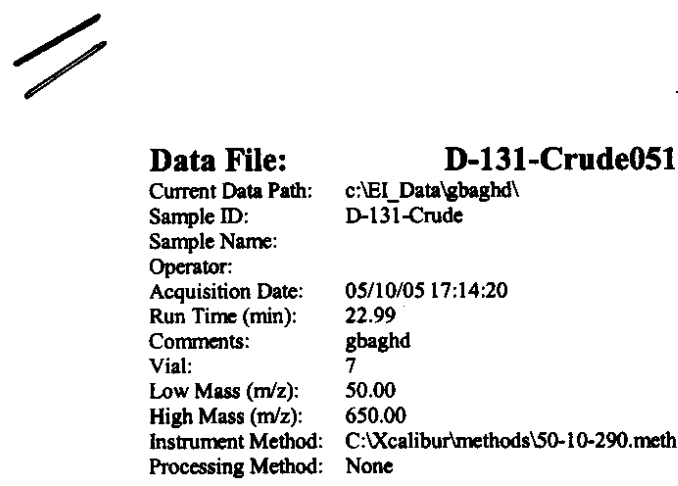

c:EI Datalgbaghd D-13i-Crude

\section{UCI MS Facility} D-131-Crude05100007_151356

RT: $0.00-26.00$ SM: $3 G$

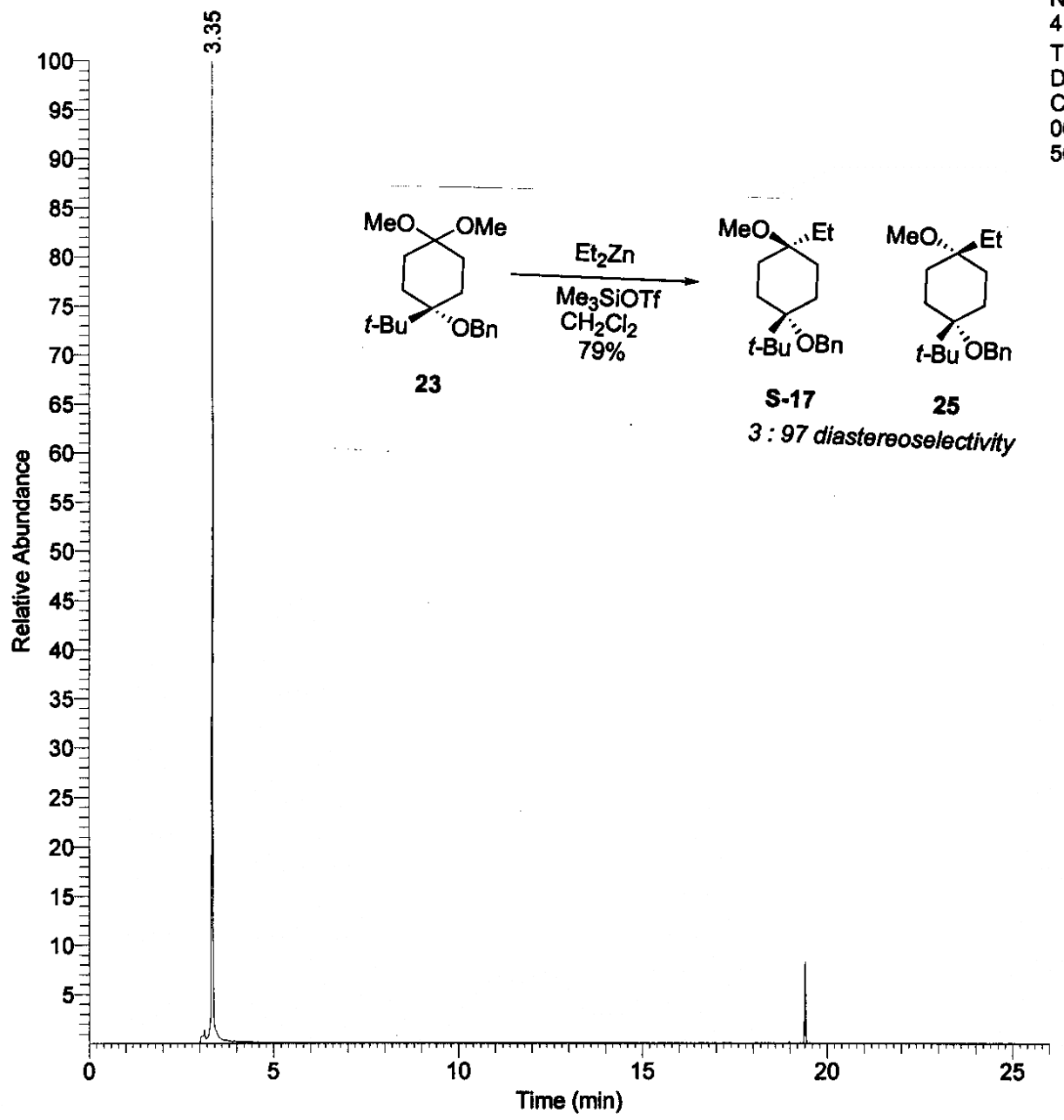

$\mathrm{NL}$

4.46E8

TIC MS

D-131-

Crude0510

$0007 \_1513$

56 
Crude05100007_151356

05/10/2005 05:14:20 PM

131-Crude05100007_151356 \#936-938 RT: 18.96-18.99 AV: 3 NL: 1.00E5 $T:\{0,0\}+c$ El det $=349.00$ Full $\mathrm{ms}$ [ 50.00-650.00]

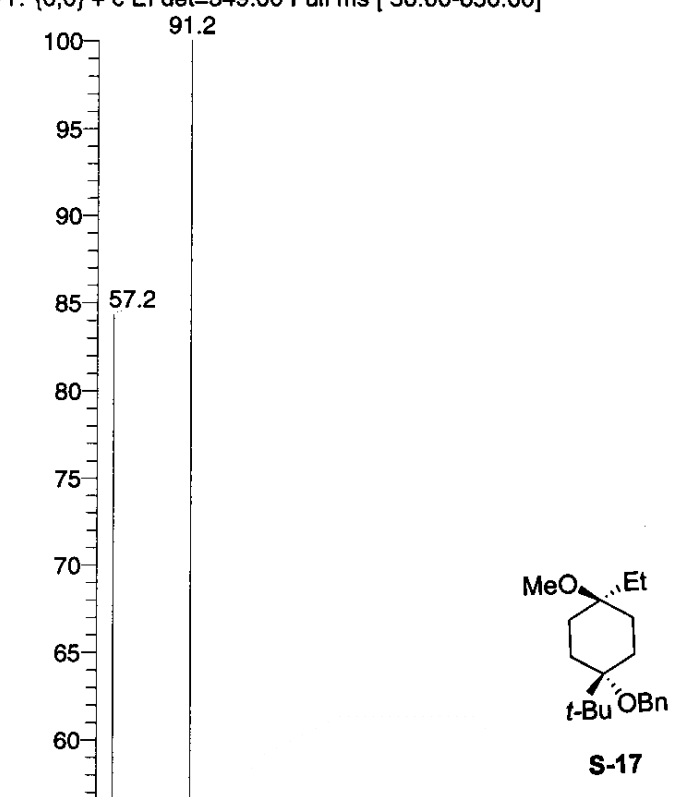

3

25

25

20

15

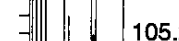

10 - 123.

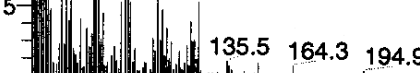

$264.0 \quad 307.0 \quad 347.5 \quad 376.7$

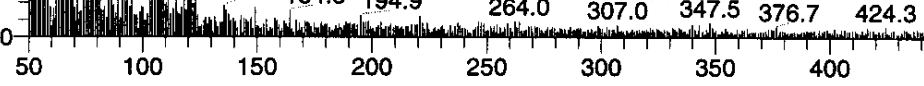

250

300

$\mathrm{m} / \mathbf{z}$ 


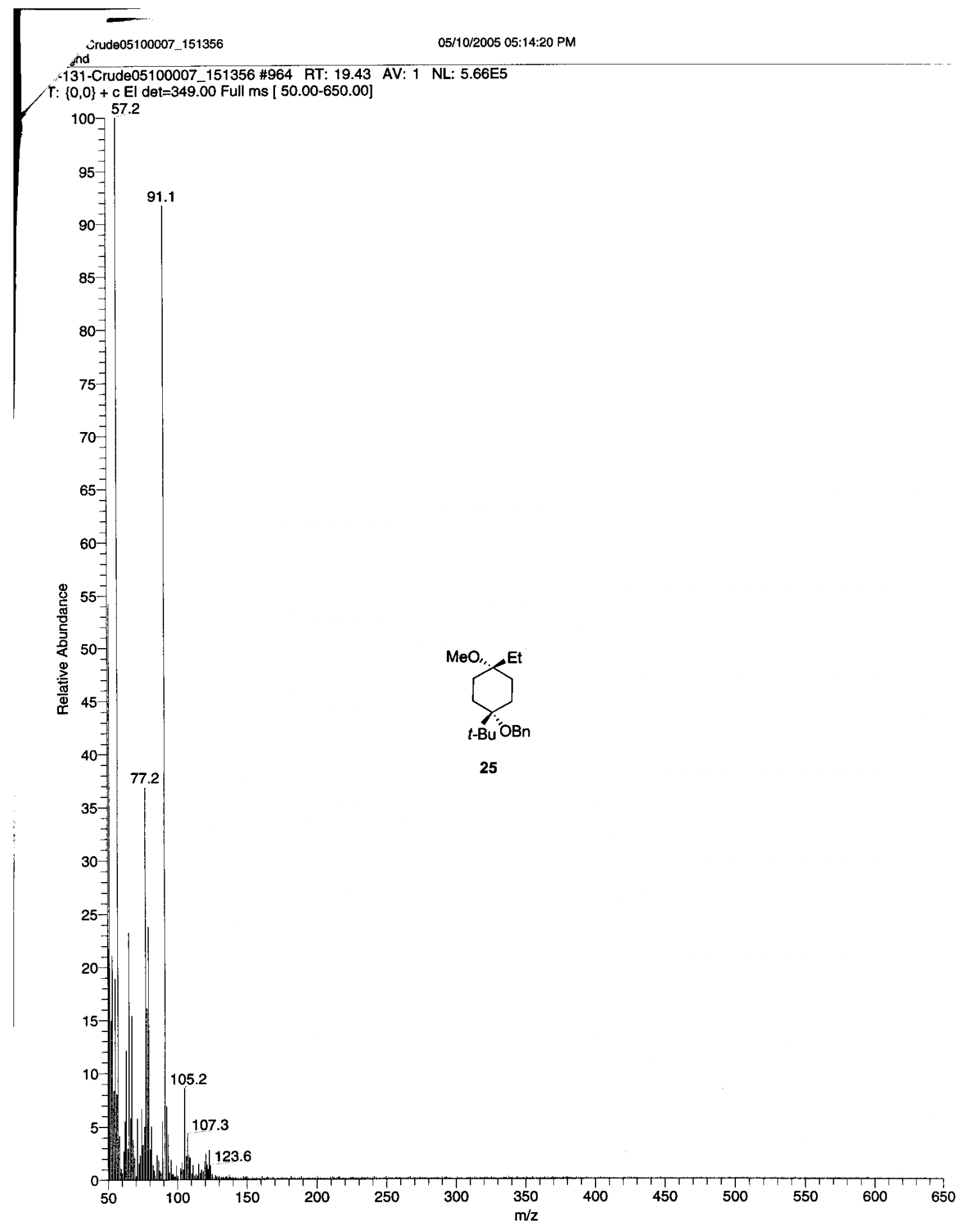




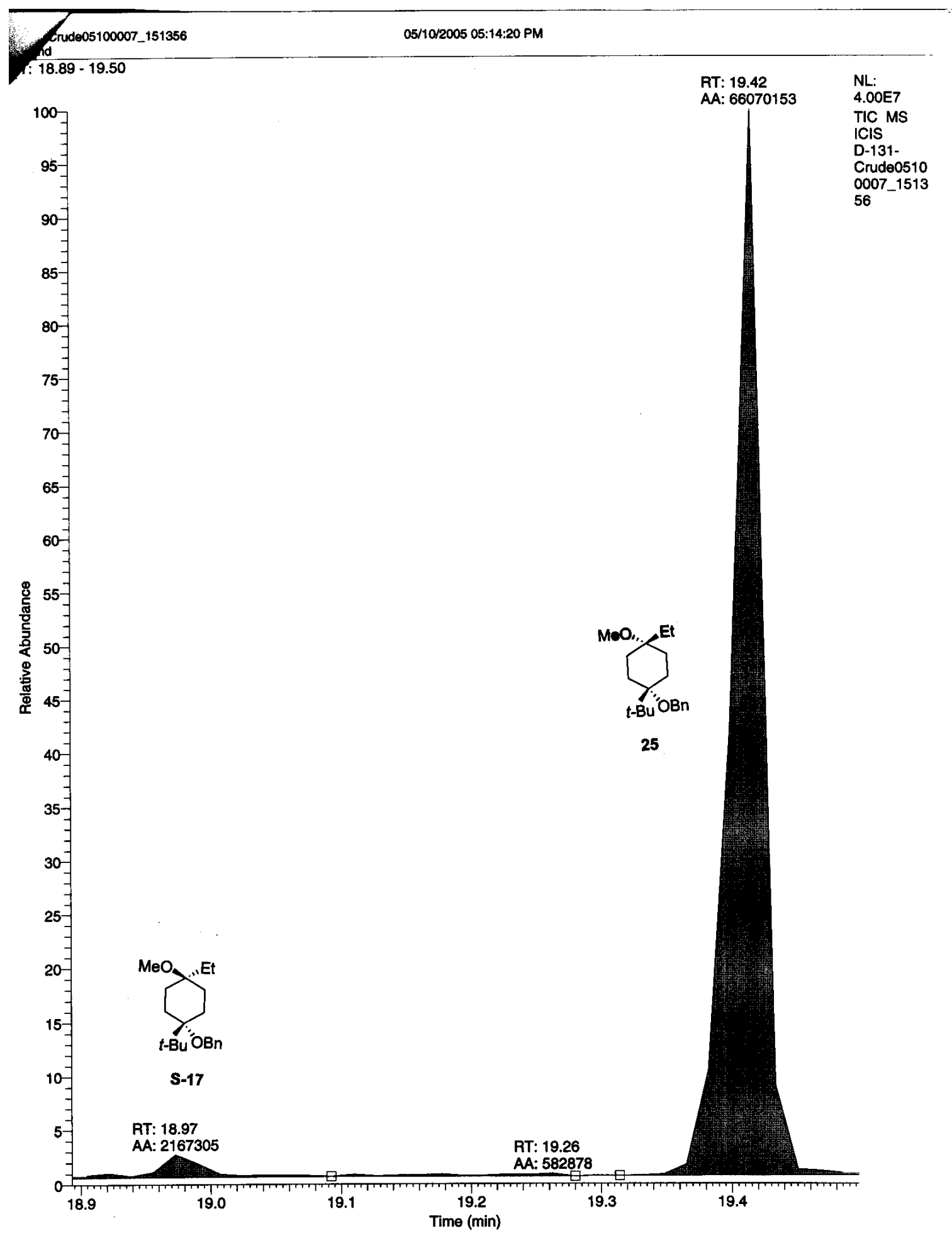


Dgat File $C: \backslash H P C H E M \backslash 1 \backslash D A T A \backslash T R \backslash 015 F 0101 . D$

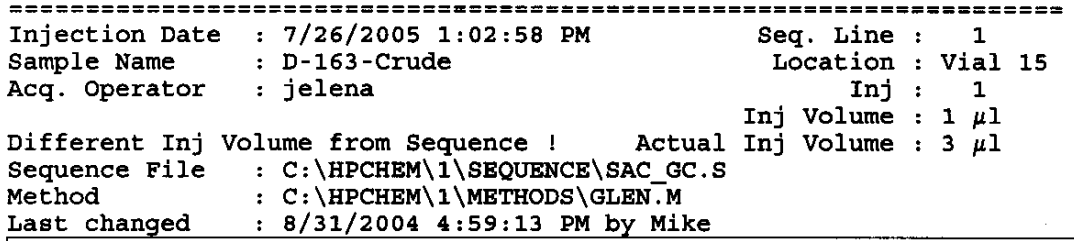

Last changed : 8/31/2004 4:59:13 PM by Mike

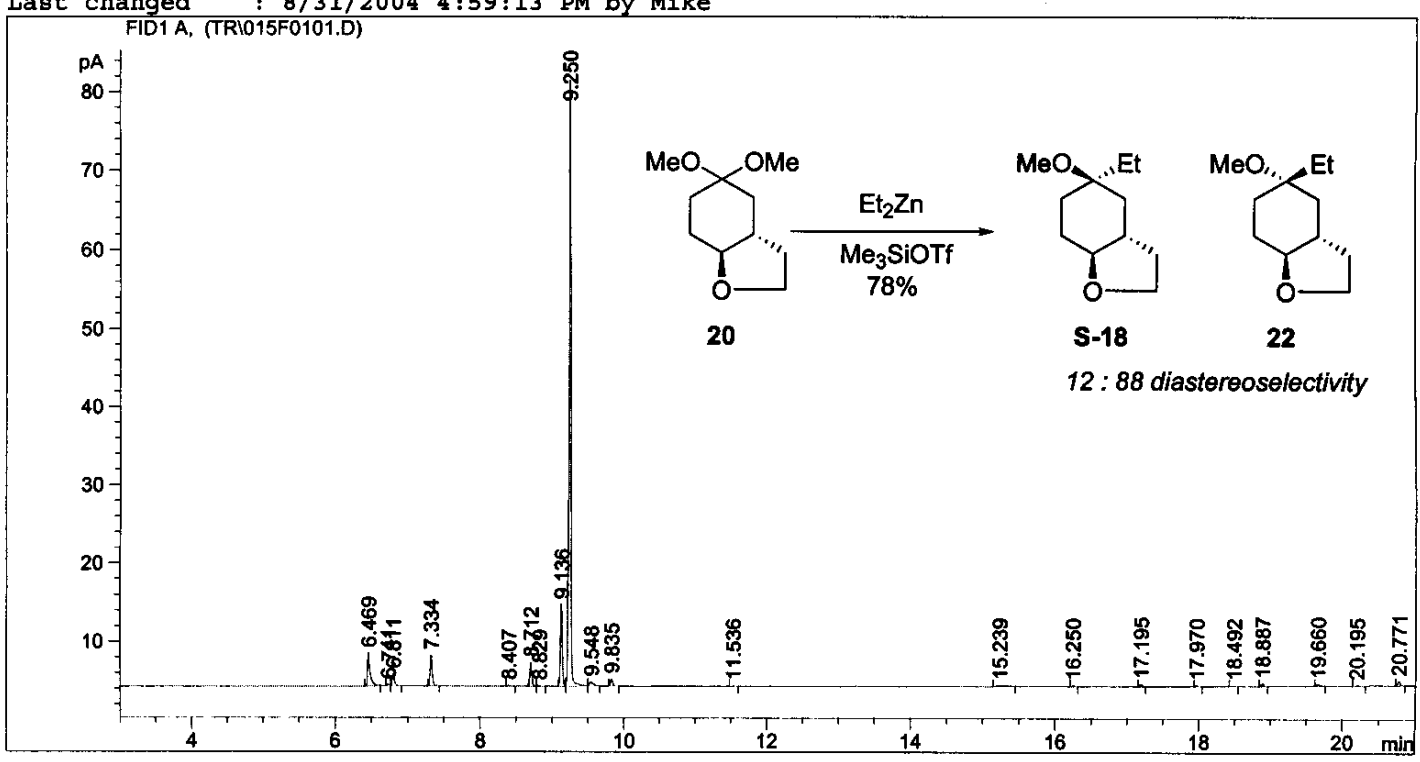

Axea Percent Report

$\begin{array}{lll}\text { Sorted By } & : & \text { Signal } \\ \text { Multiplier } & : & 1.0000 \\ \text { Dilution } & : & 1.0000\end{array}$

Signal 1: FID1 A,

\begin{tabular}{|c|c|c|c|c|c|c|}
\hline $\begin{array}{c}\text { Peak } \\
\#\end{array}$ & $\begin{array}{l}\text { RetTime } \\
\text { [min] }\end{array}$ & Type & $\begin{array}{l}\text { width } \\
\text { [min] }\end{array}$ & $\begin{array}{r}\text { Area } \\
{[\mathrm{p} A * \boldsymbol{g}]}\end{array}$ & $\begin{array}{l}\text { Height } \\
\text { [pA] }\end{array}$ & $\underset{8}{\text { Area }}$ \\
\hline & & & & & & \\
\hline $\begin{array}{l}1 \\
2\end{array}$ & $\begin{array}{l}1.418 \\
1.677\end{array}$ & $\begin{array}{l}\text { VP } \\
\text { VB }\end{array}$ & $\begin{array}{l}0.0127 \\
0.0147\end{array}$ & $\begin{array}{r}5.12484 \\
97.15041\end{array}$ & $\begin{array}{r}6.48101 \\
105.63909\end{array}$ & $\begin{array}{r}1.66806 \\
31.62114\end{array}$ \\
\hline 3 & 6.469 & PB & 0.0357 & 10.49523 & 4.27150 & 3.41605 \\
\hline 4 & 6.741 & PV & 0.0288 & $3.43706 \mathrm{e}-1$ & $1.92521 \mathrm{e}-1$ & 0.11187 \\
\hline 5 & 6.811 & VB & 0.0319 & 3.21344 & 1.53686 & 1.04593 \\
\hline 6 & 7.334 & BB & 0.0316 & 8.22469 & 3.98910 & 2.67702 \\
\hline 7 & 8.407 & BB & 0.0340 & $2.62709 \mathrm{e}-1$ & $1.15790 \mathrm{e}-1$ & 0.08551 \\
\hline 8 & 8.712 & BV & 0.0304 & 5.78648 & 3.00909 & 1.88342 \\
\hline 9 & 8.829 & VB & 0.0370 & $2.29126 \mathrm{e}-1$ & $8.61069 \mathrm{e}-2$ & 0.07458 \\
\hline 10 & 9.136 & BV & 0.0296 & 19.75997 & 10.43944 & 6.43160 \\
\hline 11 & 9.250 & VB & 0.0292 & 146.99409 & 77.18916 & 47.84458 \\
\hline 12 & 9.548 & BB & 0.0472 & 1.91848 & $5.63738 e-1$ & 0.62444 \\
\hline 13 & 9.835 & VB & 0.0326 & 1.94985 & $9.07069 \mathrm{e}-1$ & 0.63465 \\
\hline 14 & 11.536 & PB & 0.0329 & $2.14106 e-1$ & $1.02292 \mathrm{e}-1$ & 0.06969 \\
\hline 15 & 15.239 & PB & 0.1030 & 1.07467 & $1.22876 \mathrm{e}-1$ & 0.34979 \\
\hline 16 & 16.250 & PB & 0.0405 & $2.96700 \mathrm{e}-1$ & $1.13368 \mathrm{e}-1$ & 0.09657 \\
\hline
\end{tabular}




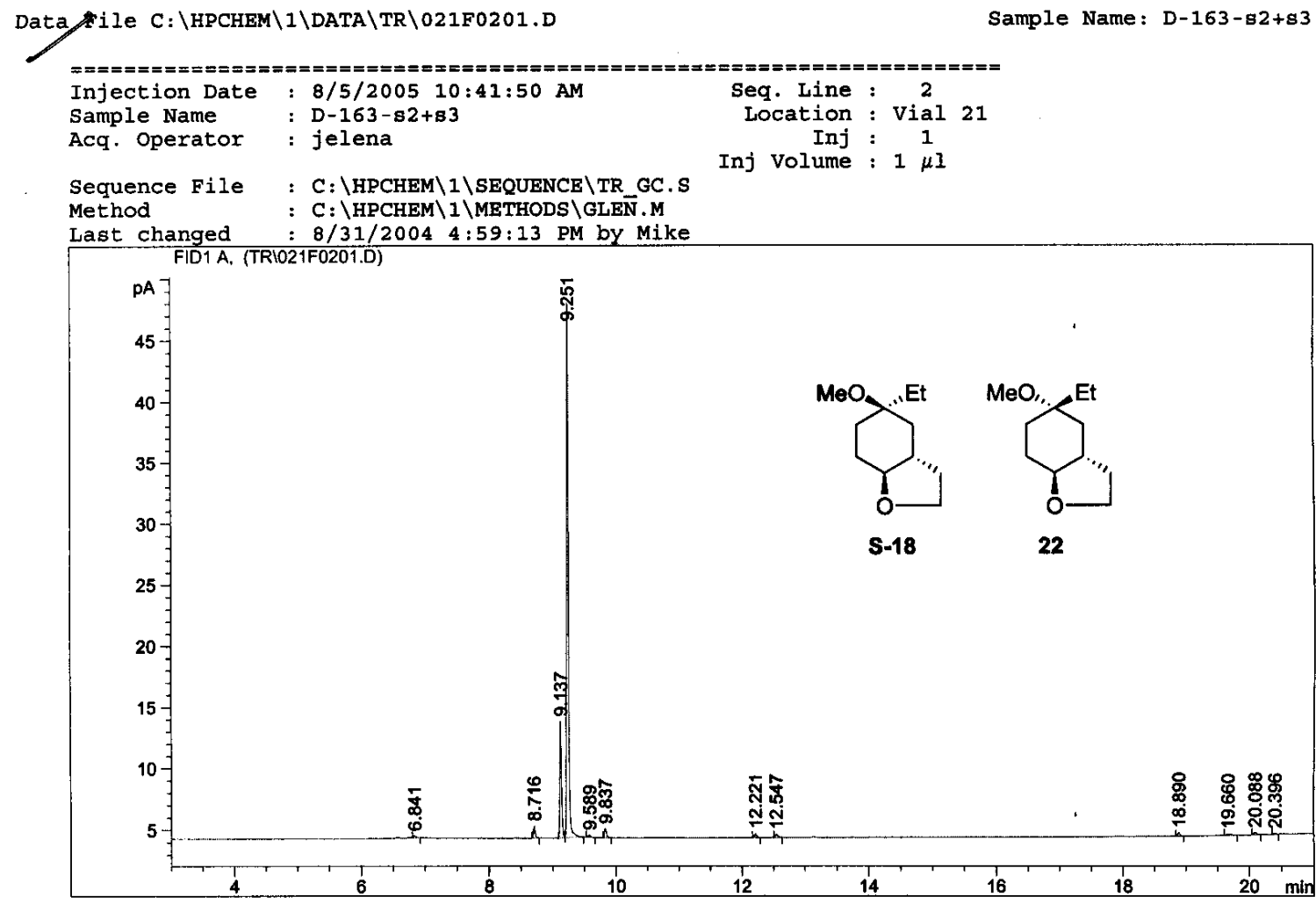

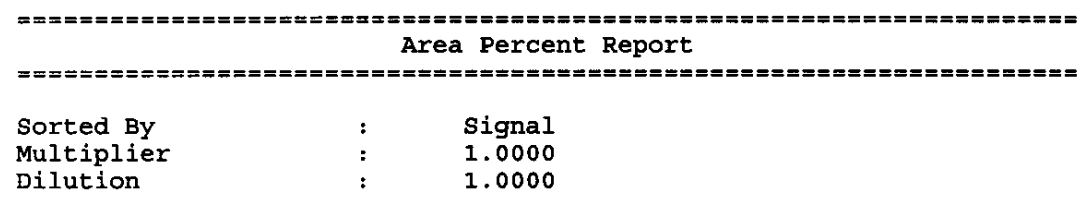

Signal 1: FID1 A,

\begin{tabular}{|c|c|c|c|c|c|c|}
\hline $\begin{array}{c}\text { Peak } \\
\#\end{array}$ & $\begin{array}{l}\text { RetTime } \\
\text { [min] }\end{array}$ & Type & $\begin{array}{l}\text { Width } \\
\text { [min] }\end{array}$ & $\begin{array}{r}\text { Area } \\
{\left[\mathrm{pA}^{*} \mathrm{~B}\right]}\end{array}$ & $\begin{array}{l}\text { Height } \\
{[\mathrm{pA}]}\end{array}$ & $\begin{array}{c}\text { Area } \\
\frac{8}{\gamma}\end{array}$ \\
\hline & & & & & & ----- \\
\hline 1 & 1.418 & PB & 0.0127 & $7.05289 e-1$ & $8.97078 e-1$ & 0.51371 \\
\hline 2 & 1.677 & PB & 0.0150 & 23.32384 & 24.62637 & 16.98820 \\
\hline 3 & 6.841 & VB & 0.0414 & $4.58482 e-1$ & $1.46950 \mathrm{e}-1$ & 0.33394 \\
\hline 4 & 8.716 & PB & 0.0302 & 1.81768 & $9.55603 \mathrm{e}-1$ & 1.32393 \\
\hline 5 & 9.137 & PV & 0.0287 & 17.56937 & 9.45202 & 12.79686 \\
\hline 6 & 9.251 & VB & 0.0311 & 88.07758 & 43.61605 & 64.15235 \\
\hline 7 & 9.589 & BP & 0.0355 & $4.12698 \mathrm{e}-1$ & $1.75257 \mathrm{e}-1$ & 0.30059 \\
\hline 8 & 9.837 & BB & 0.0351 & 1.63731 & $7.17562 \mathrm{e}-1$ & 1.19255 \\
\hline 9 & 12.221 & $\mathrm{BB}$ & 0.0349 & $6.95677 e-1$ & $3.19152 \mathrm{e}-1$ & 0.50670 \\
\hline 10 & 12.547 & $\mathrm{BB}$ & 0.0388 & $6.72007 e-1$ & $2.46930 \mathrm{e}-1$ & 0.48946 \\
\hline 11 & 18.890 & PB & 0.0383 & $6.64257 e-1$ & $2.60244 \mathrm{e}-1$ & 0.48 \\
\hline 12 & 19.660 & PB & 0.0701 & $5.17918 e-1$ & $9.50166 e-2$ & 0.37723 \\
\hline 13 & 20.088 & PB & 0.0414 & $5.18793 e-1$ & $1.76041 \mathrm{e}-1$ & 0.37787 \\
\hline 14 & 20.396 & $\mathrm{BP}$ & 0.0377 & $2.23491 \mathrm{e}-1$ & $8.78507 \mathrm{e}-2$ & 0.16278 \\
\hline & & & & 137.29439 & 81. & \\
\hline
\end{tabular}




\section{UCI MS Facility}

Data File:

Current Data Patt

D-140-Crude2ndtry06020081_122400

Sample ID:

Sample Name:

Operator:

Acquisition Date: $\quad 06 / 02 / 2005$ 12:49:45 PM

Run Time ( $\min$ ): $\quad 22.98$

Comments: gbaghd

Vial:

Low Mass $(\mathrm{m} / \mathrm{z})$ :

High Mass $(\mathrm{m} / \mathrm{z})$ :

Instrument Method: E:Kcaliburtmethods 50 -10-290.meth

Processing Method: None
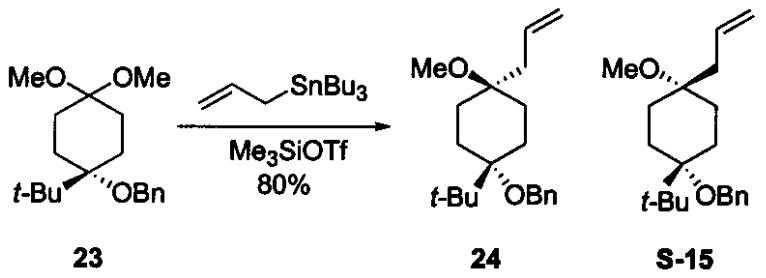

RT: $0.00-25.98$ SM: $3 G$

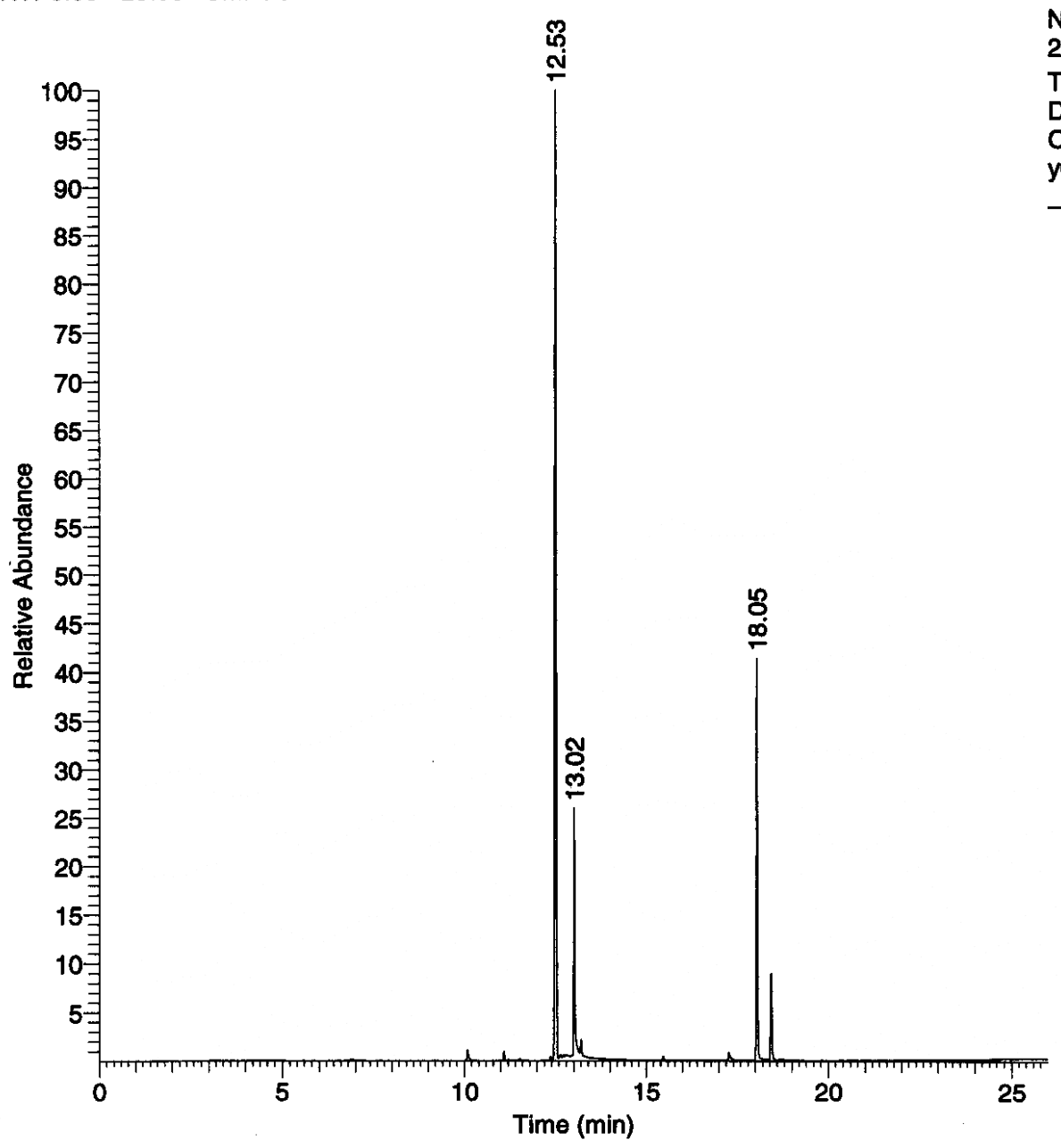

NL:

2.89E9

TIC MS

D-140-

Crude2ndtr

y06020081

$-122400$

Page 1 of 4 


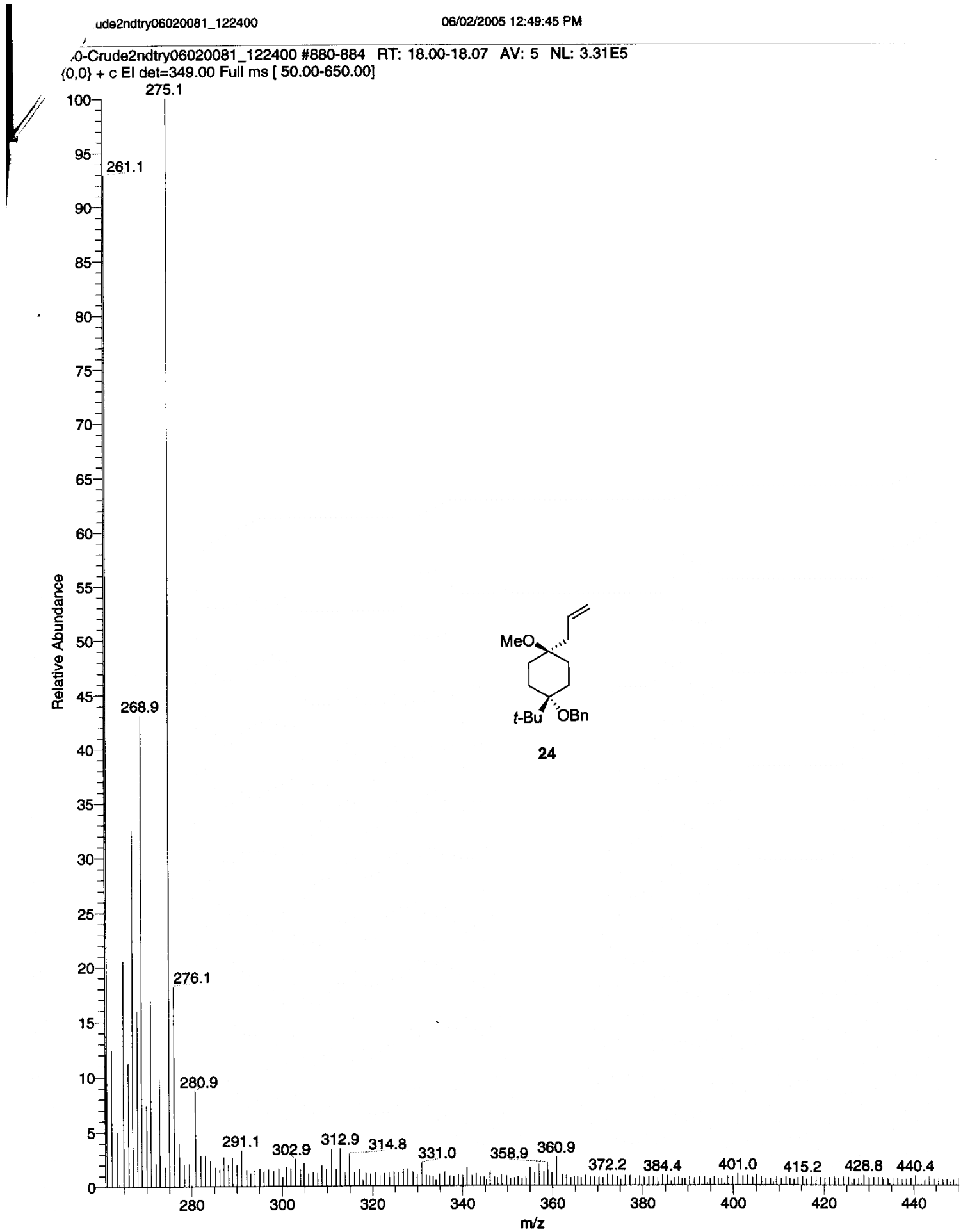




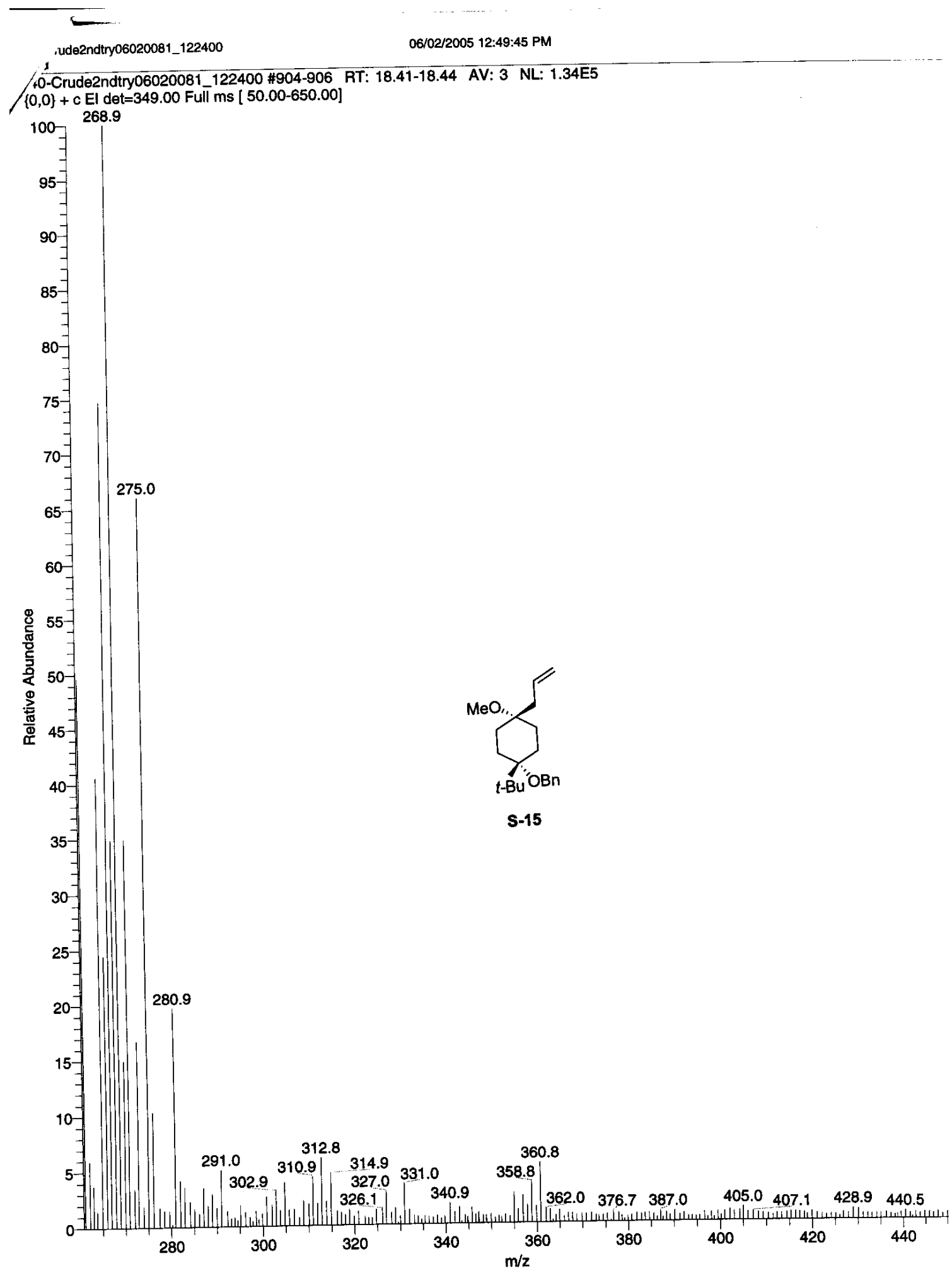




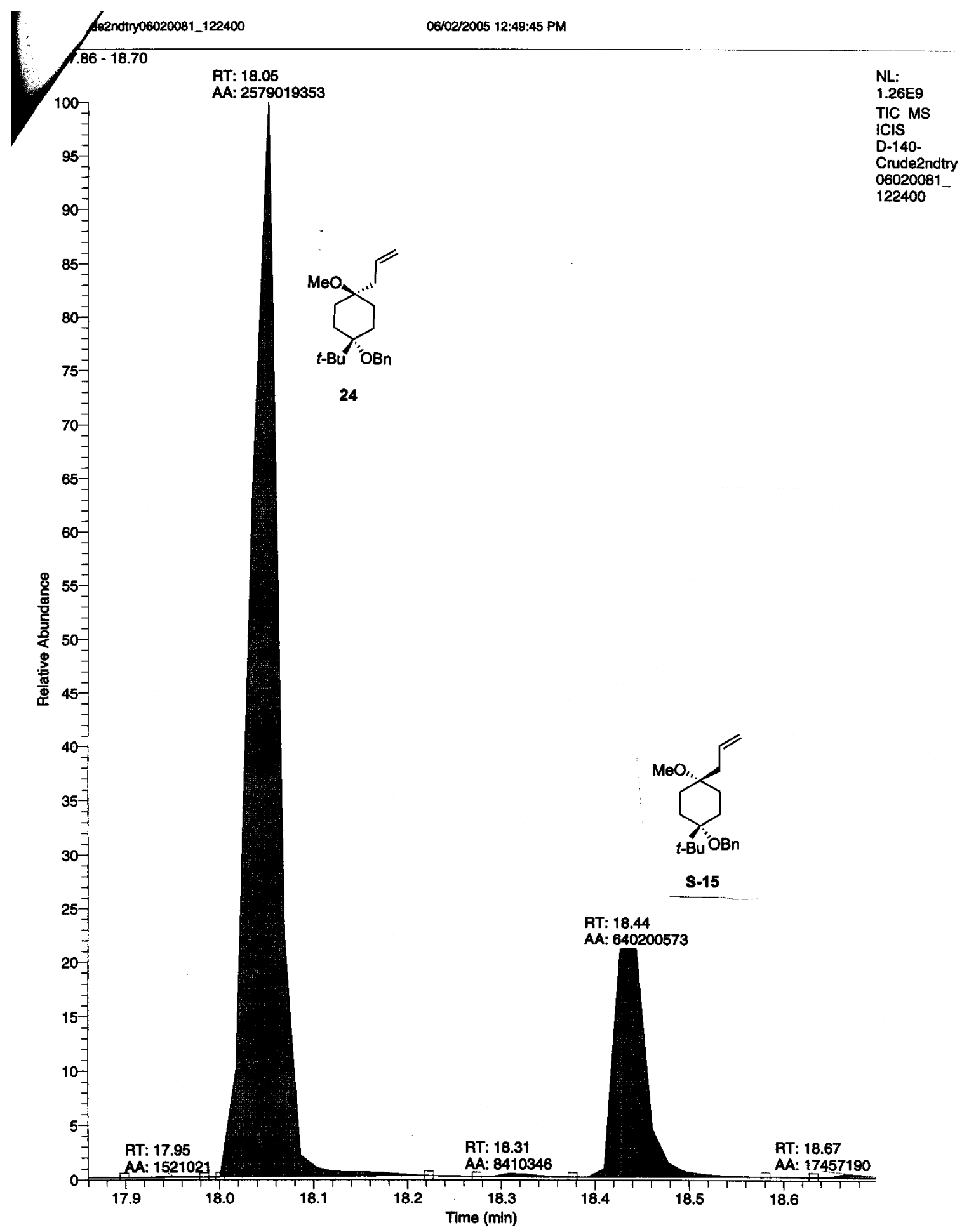

T 


\section{Correlation of $6 b$ and $6 b^{\prime}$}

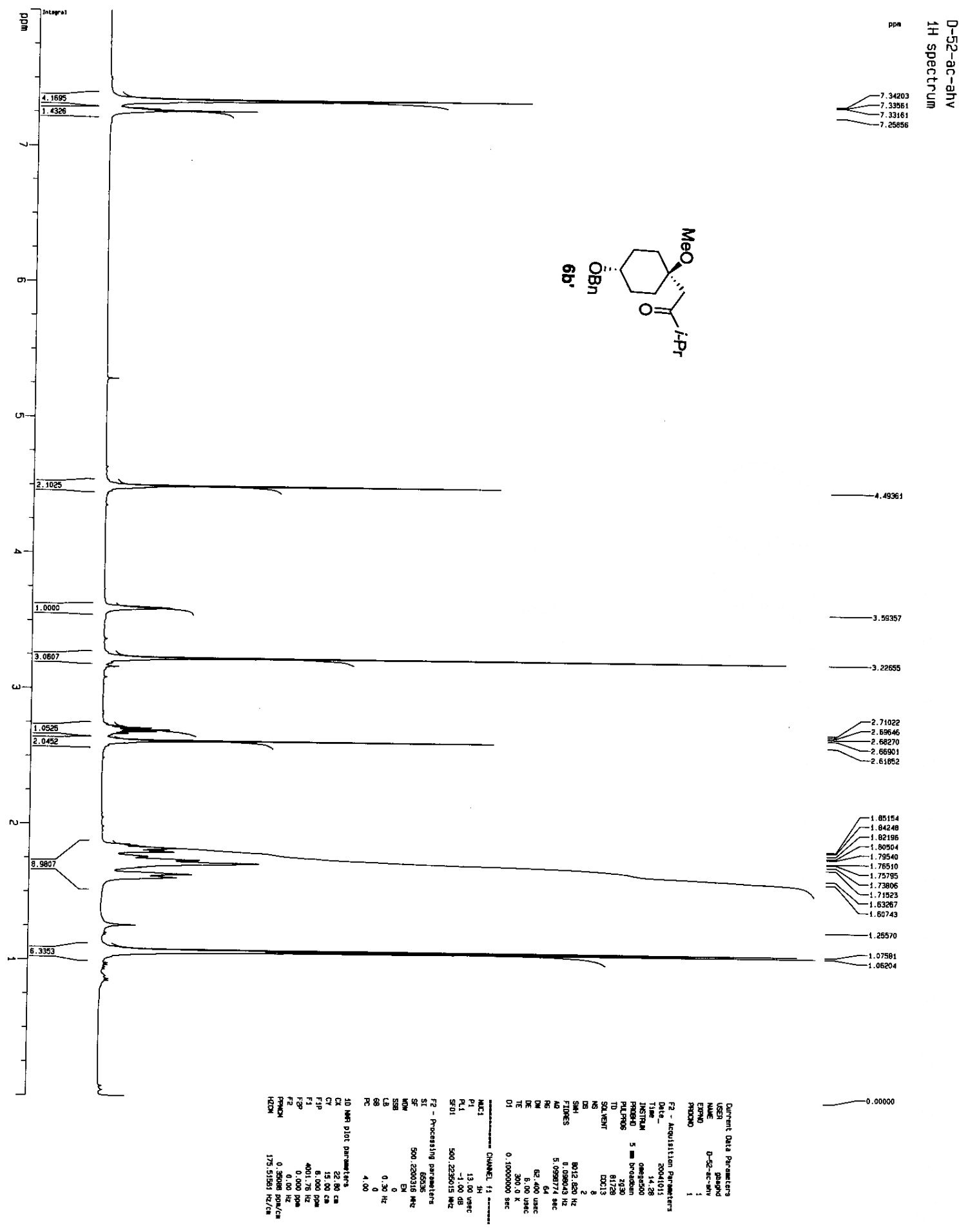




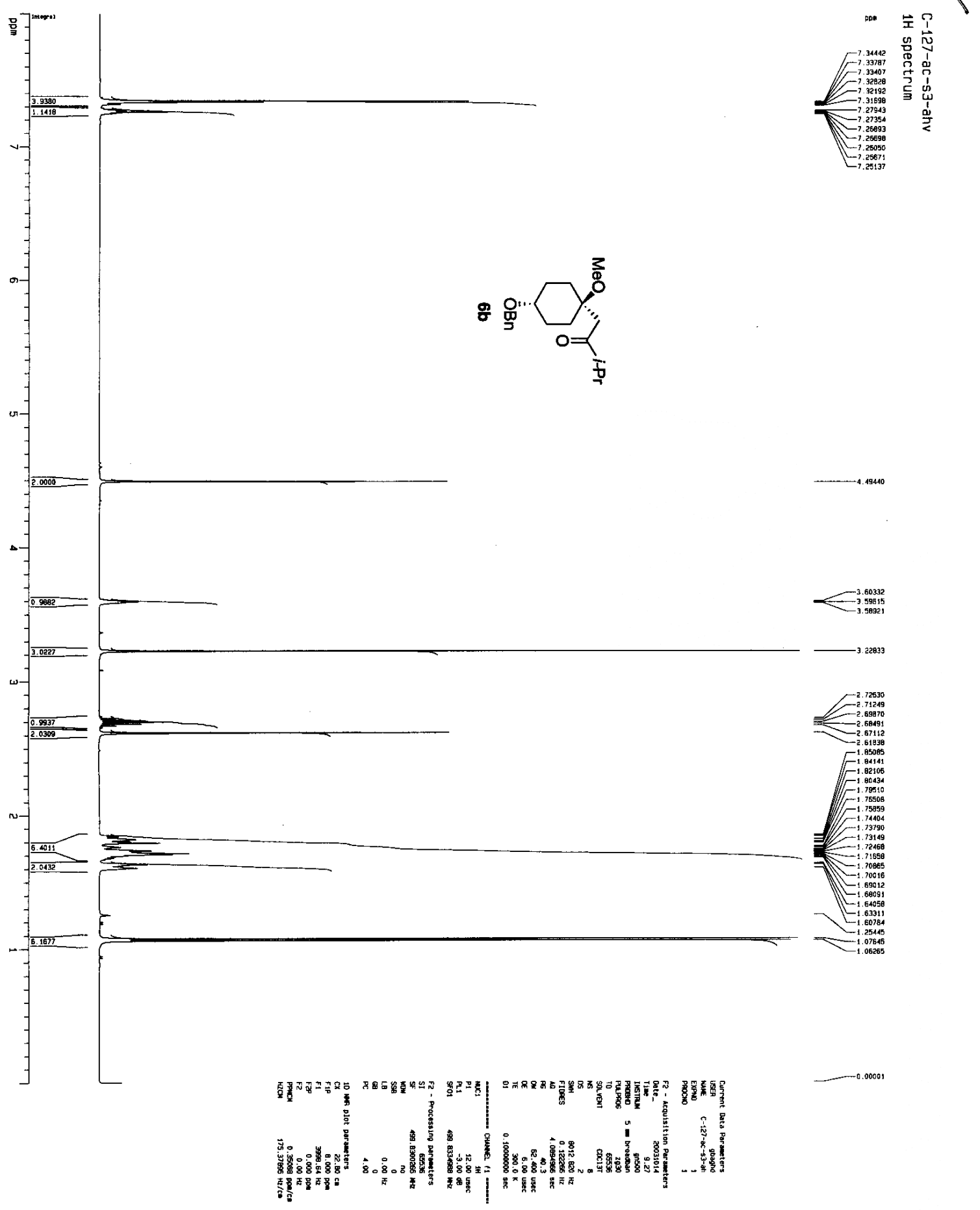




\section{Proofs of Purity}

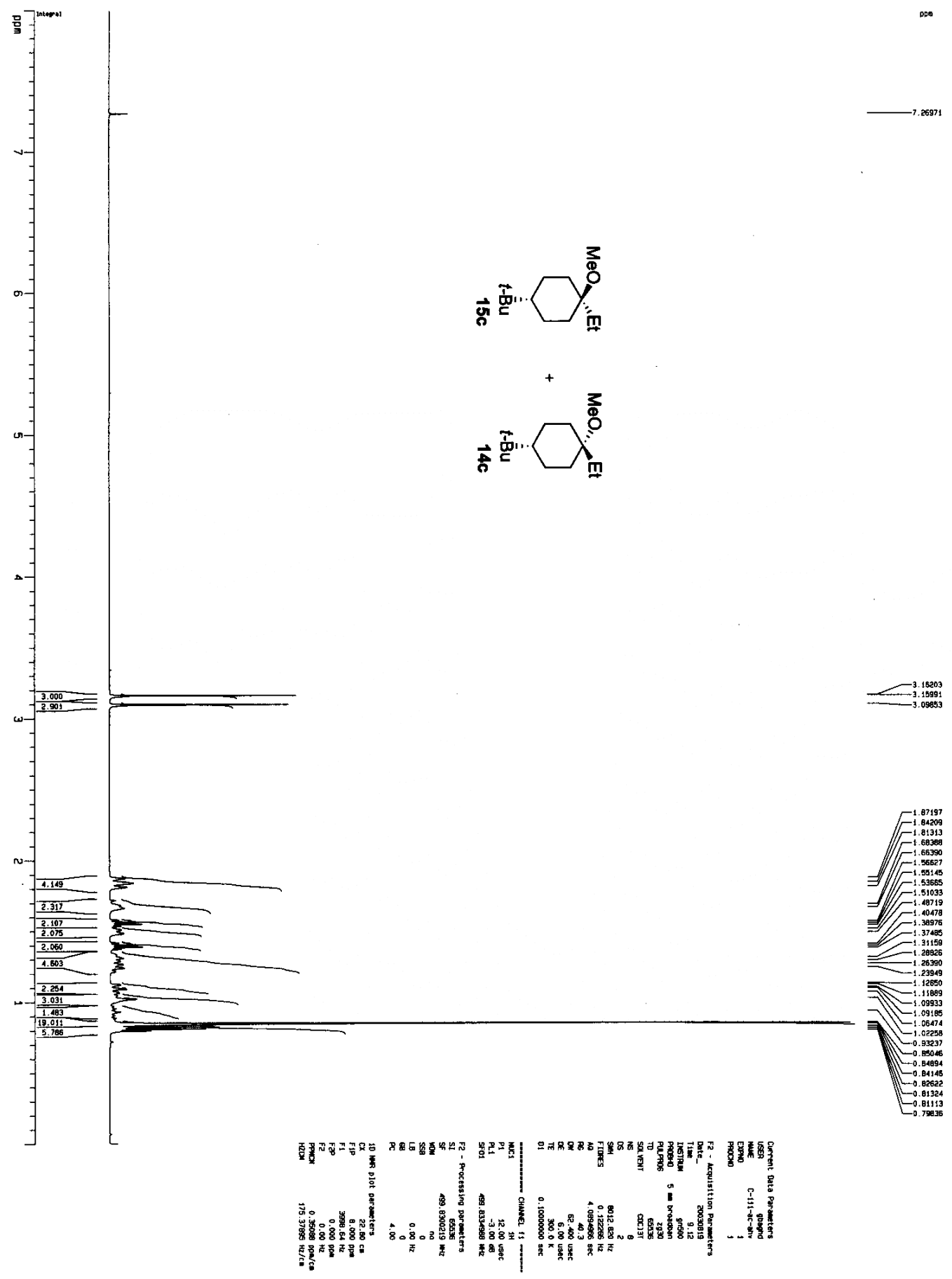




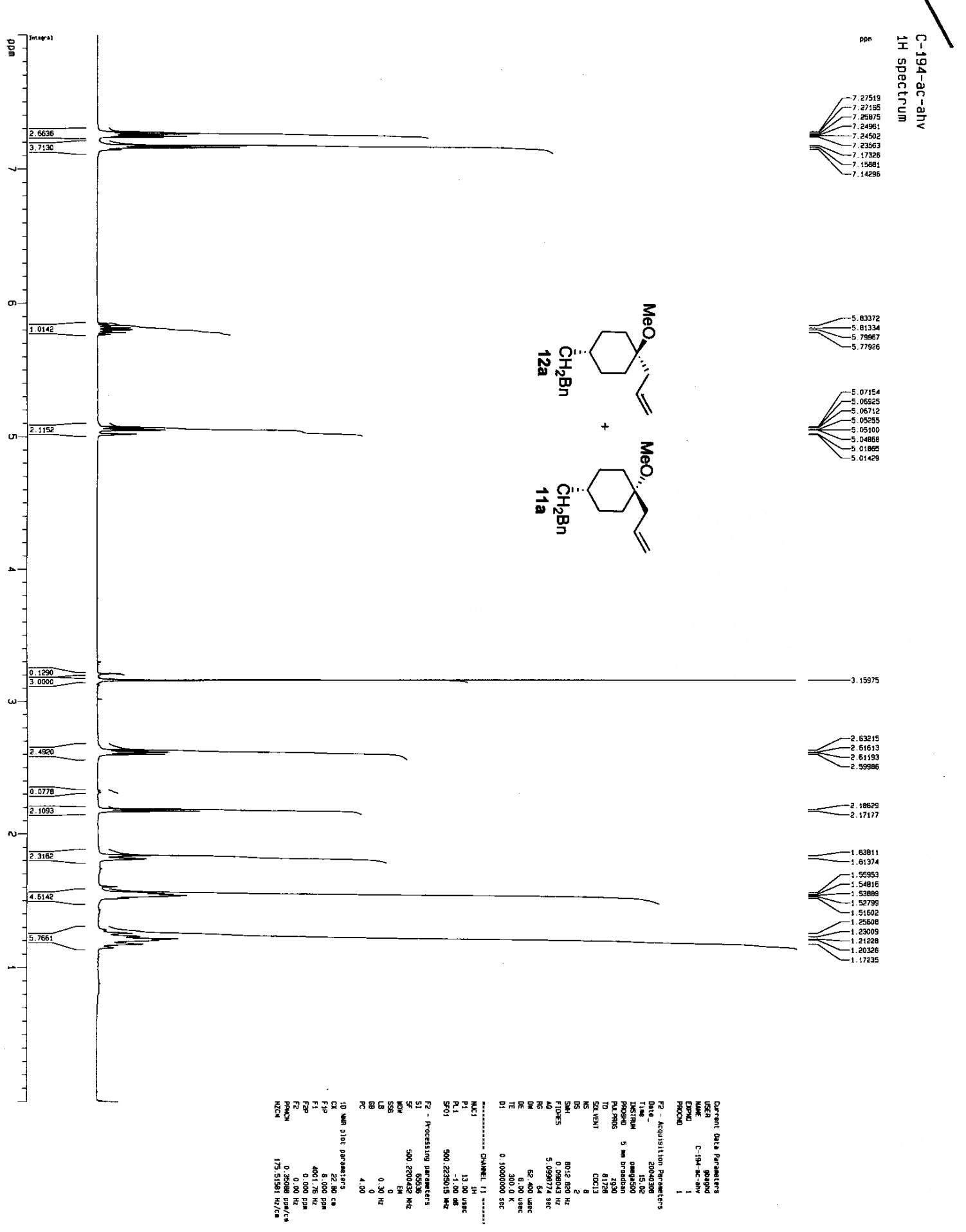




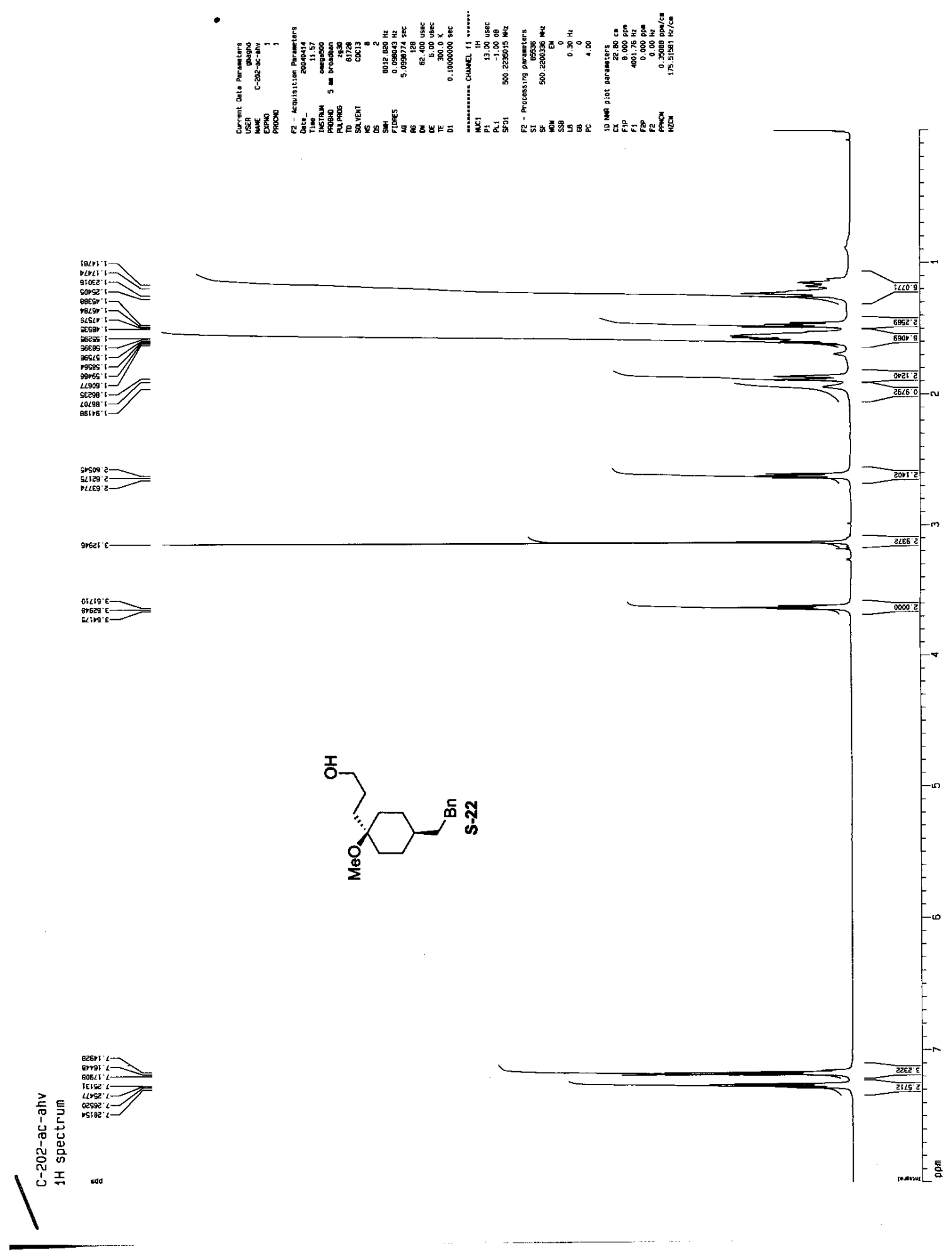




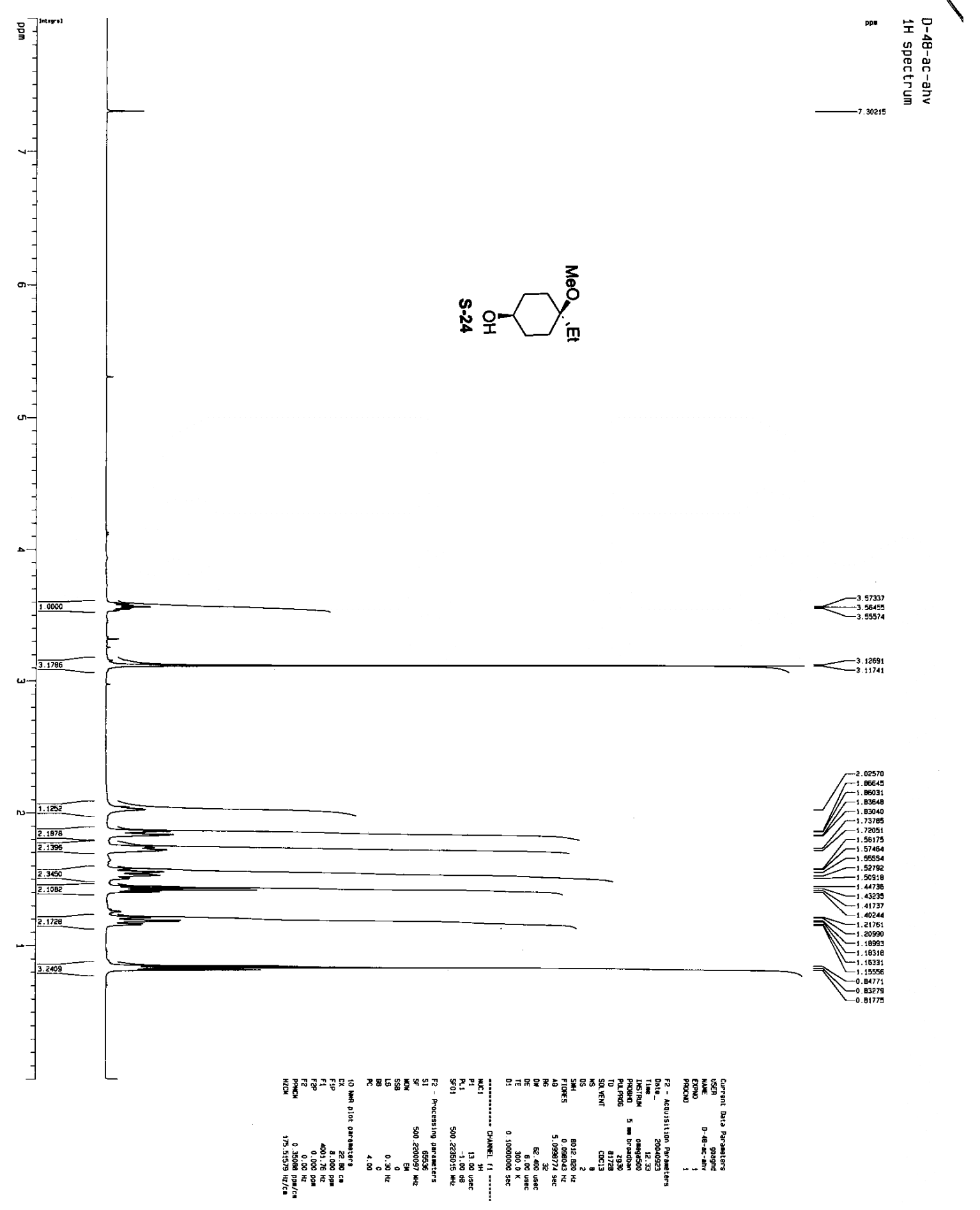




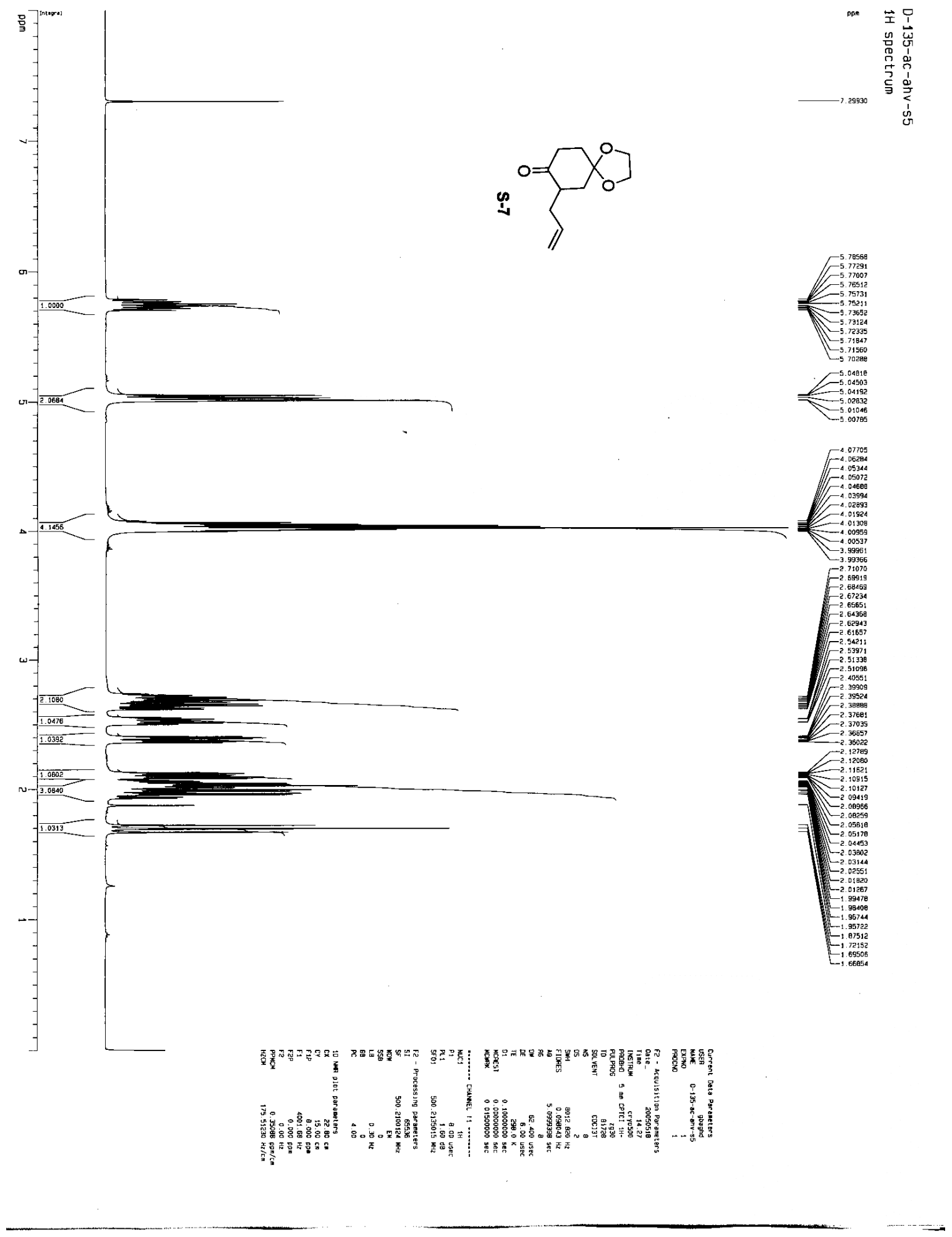




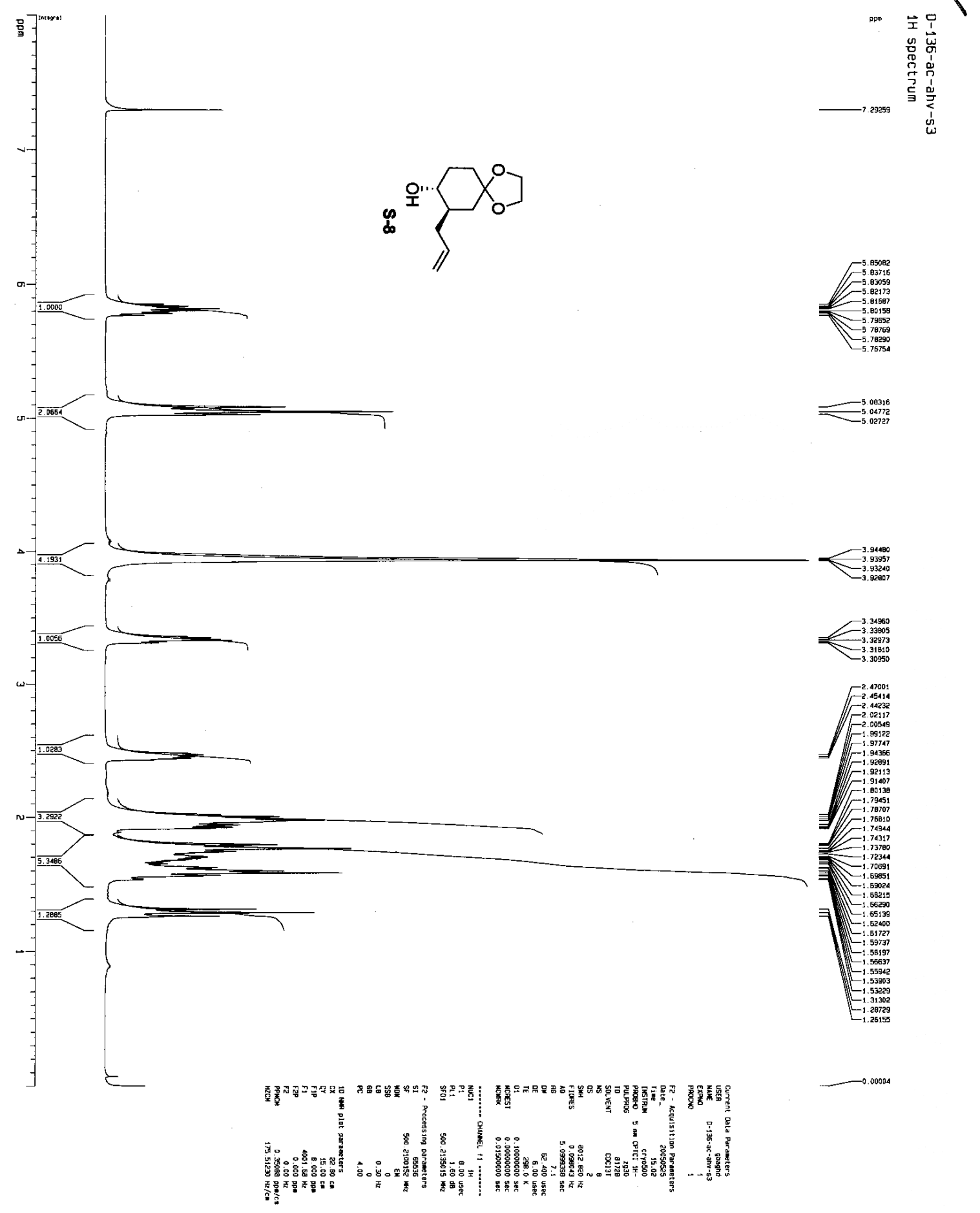




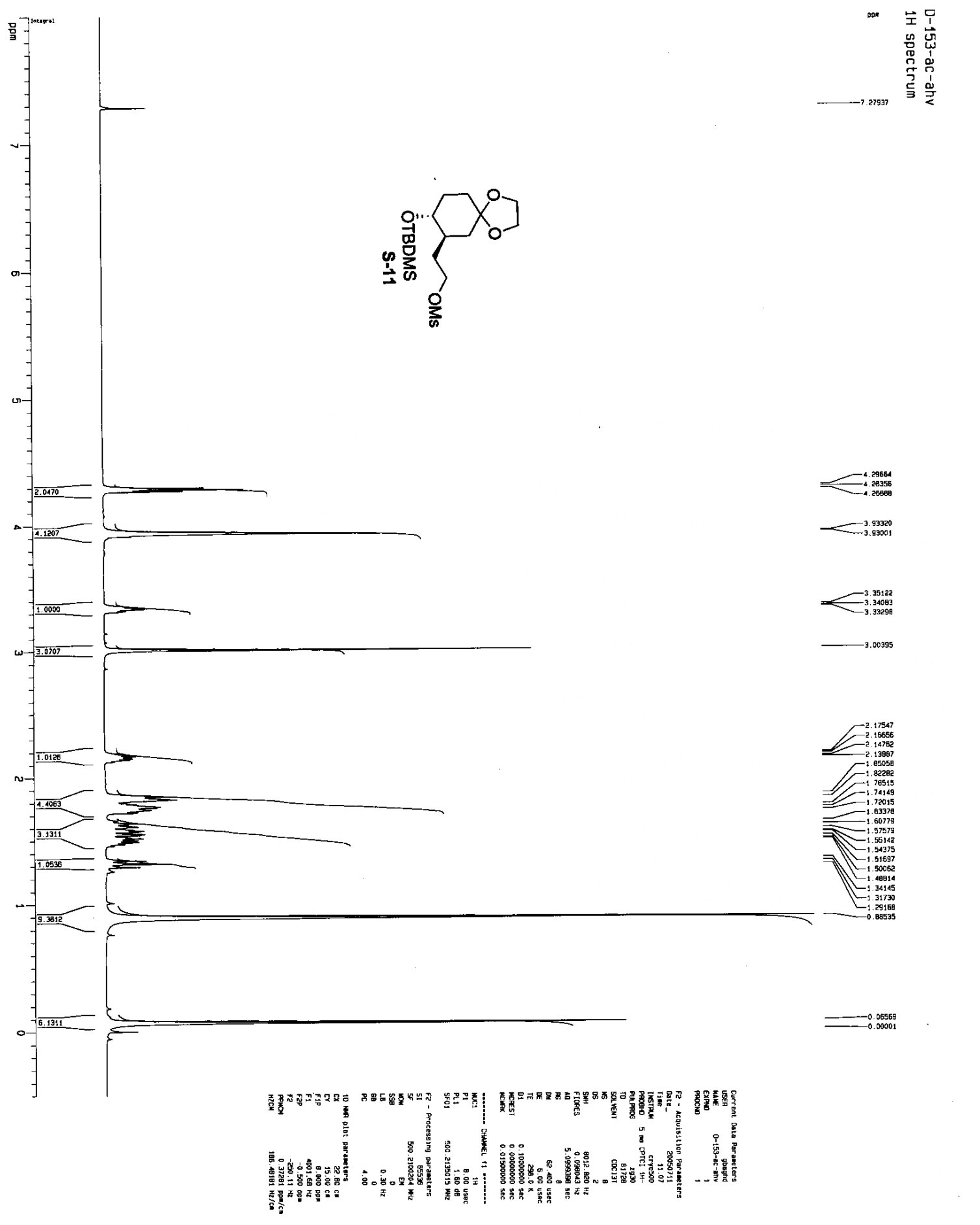




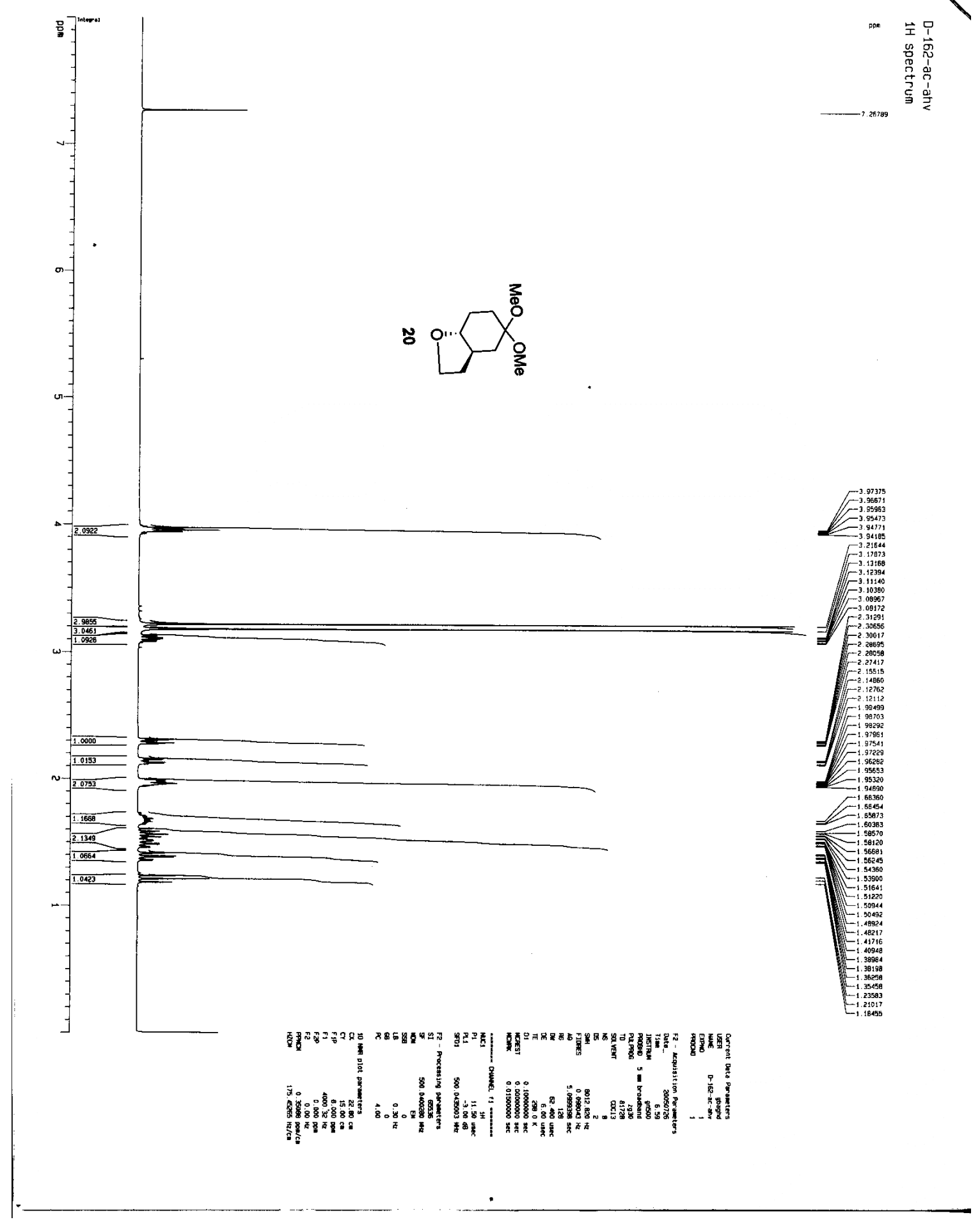

